Westinghouse Electric Corporation Fusion Power Systems Department

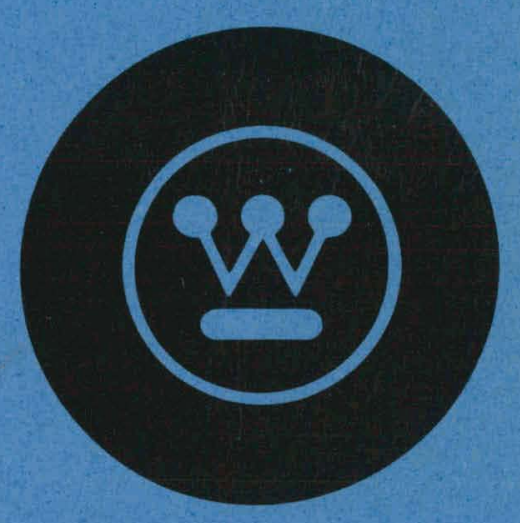

COMPILATION OF TFTR MATERIALS DATA 


\section{DISCLAIMER}

This report was prepared as an account of work sponsored by an agency of the United States Government. Neither the United States Government nor any agency Thereof, nor any of their employees, makes any warranty, express or implied, or assumes any legal liability or responsibility for the accuracy, completeness, or usefulness of any information, apparatus, product, or process disclosed, or represents that its use would not infringe privately owned rights. Reference herein to any specific commercial product, process, or service by trade name, trademark, manufacturer, or otherwise does not necessarily constitute or imply its endorsement, recommendation, or favoring by the United States Government or any agency thereof. The views and opinions of authors expressed herein do not necessarily state or reflect those of the United States Government or any agency thereof. 


\section{DISCLAIMER}

Portions of this document may be illegible in electronic image products. Images are produced from the best available original document. 
WFPS -TME - 031

DECEMBER 1975

\section{COMPILATION OF TFTR MATERIALS DATA}

Prepared by: W. J. Havener

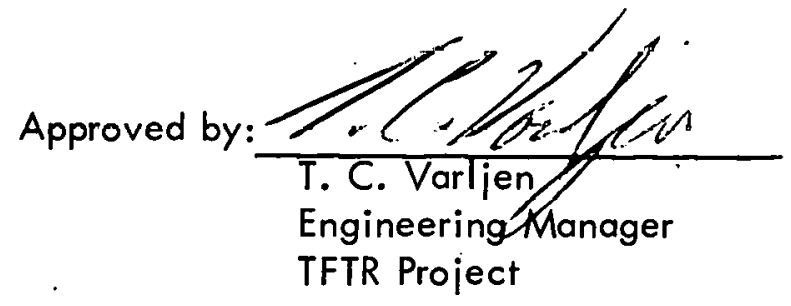

$$
\begin{aligned}
& \text { Approved by: } C-C \\
& \text { C-K. Kim, Manager } \\
& \text { Systems Engineering \& Analysis } \\
& \text { TFTR Project }
\end{aligned}
$$

Westinghouse Electric Corporation Fusion Power Systems Department

P. O. Box 10864

Pittsburgh, Pennsylvania 15236

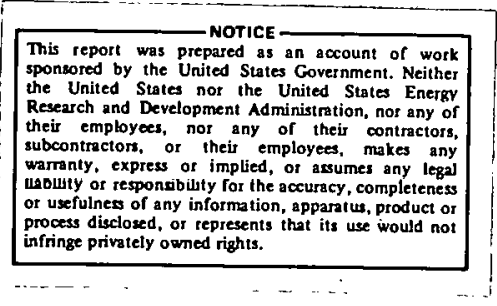




\begin{abstract}
In order to document the key thermophysical property data used in the conceptual design of Tokamak Fusion Test Reactor (TFTR) systems and components, a series of data packages has been prepared. It is expected that data for additional materials will be added and the information already provided will be updated to provide a project-wide data base.
\end{abstract}

\title{
eUtiac ins?
}

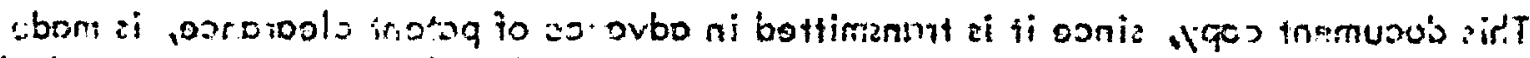

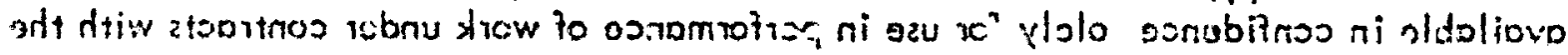

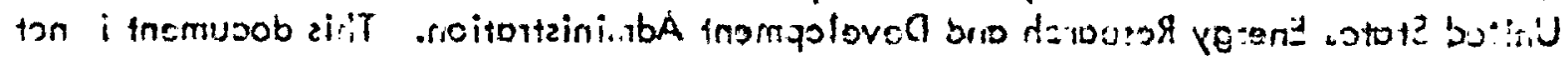

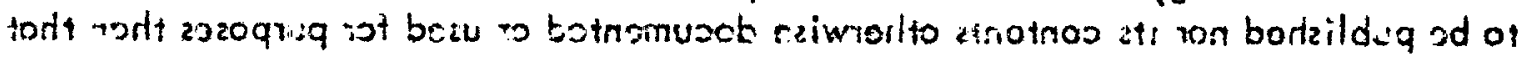

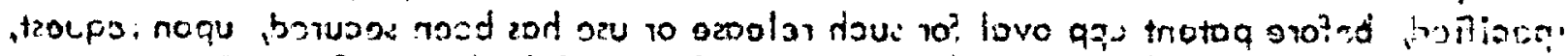

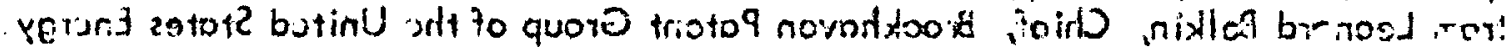

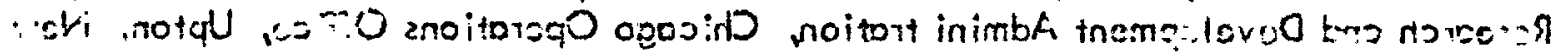




\section{ACKNOWLEDGEMENT}

This work was performed for the Plasma Physics Laboratory, Princeton University, under U. S. Energy Research and Development Administration Contract E(11-1)-3073, Subcontract 192. Reproduction, translation, publication, use and disposal, in whole or in part, by or for the United States Government is permitted.

\section{LEGAL NOTICE}

This report was prepared as an account of Government sponsored work. Neither the United States, nor the Administration, nor any person acting on behalf of the Administration:

A. Makes any warranty or representation, express or implied, with respect to the accuracy, completeness, or usefulness of the information contained in this report, or that the use of any information, apparatus, method or process disclosed in this report may not infringe privately owned rights; or

B. Assumes any liabilities with respect to the use of, or for damages resulting from the use of any information, apparatus, method, or process disclosed in this report.

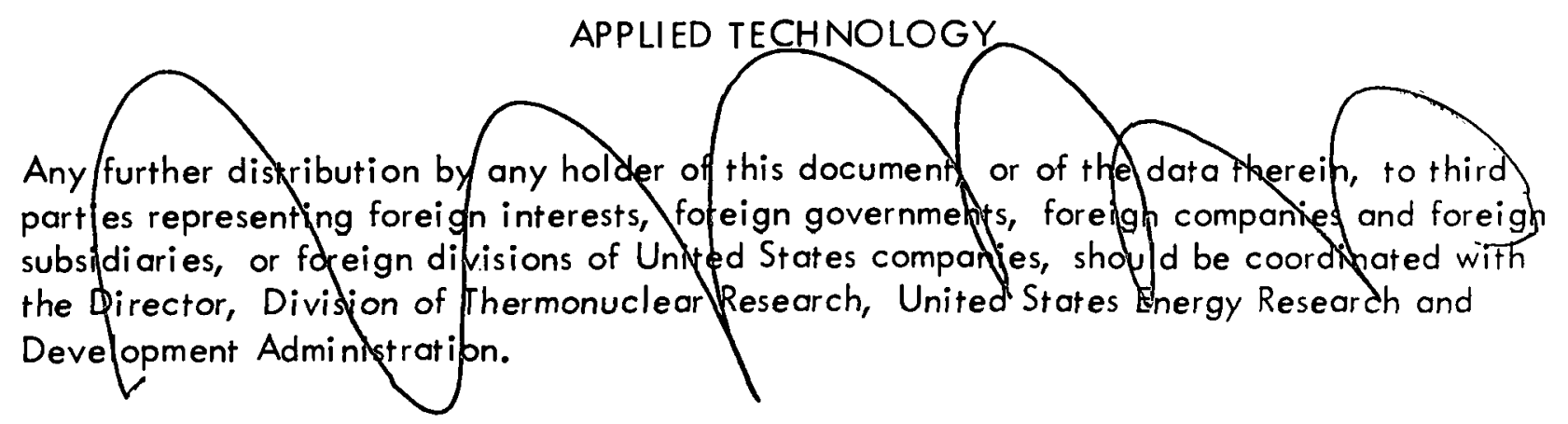




\subsection{INTRODUCTION}

This report contains a compilation of some of the materials properties that have been used in the Tokamak Fusion Test Reactor (TFTR) Conceptual Design efforts. This compilation is certainly not definitive, documentation is provided for materials data used to date. A basis has been provided for a future project-wide materials data base which will be considerably widened in scope over the information presented herein.

A number of the curves included in the sections of this report are from materials data books prepared by Westinghouse for other projects, but the data are referenced to the original sources, which are more generally accessible. Other data is from sources researched in preparing this report. The data on any particular material is by no means complete but contains basic information which, it is expected, will be added to as the project progresses and the information is required and/or developed.

The minimum expected values for ultimate and yield strengths are identical to those used by the ASME Boiler and Pressure Vessel Code to establish allowable stresses. It is assumed in the Code that $95 \%$ of the data points will be above the minimum curve. Data indicated as average is that in which there was sufficient data available within a $\pm 10 \%$ band to permit the use of an averaging process to prepare a single curve for ease in usirly the data. Some curves are $80 \%$ of the average values and are so stated.

Tritium properties have been compiled and reported in TME-004, "Tritium Properties and Related Quantities Used in the TFTR Conceptual Design," by H. J. Garber. 
TABLE OF CONTENTS

MATERIAL DATA PACKAGES

1.0 INTRODUCTION

2.0 304 STAINLESS STEEL

$3.0 \quad 305$ STAINLESS STEEL

4.0 316 STAINLESS STEEL

$5.0 \quad$ NITRONIC 33

6.0 MOLYBDENUM

7.0 MOLYBDENUM - TZM

8.0 TUNGSTEN

9.0 OXYGEN FREE COPPER

10.0 ELECTRICAL COIL INSULATION 
SECTION 2.0

304 STAINLESS STEEL 


\section{TABLE OF CONTENTS}

$\underline{\text { Title }}$

1.0 BASIC DATA

I.I GENERAL

1.2 COMMERCIAL DESIGNATIONS

1.3 CHEMICAL COMPOSITION (WT.\%)

I.4 ROOM TEMPERATURE PROPERTIES

1.4. 1 Mechanical Properties (Tensile, Ultimate)

1.4.2 Physical Properties (Density, Spec. Ht.)

1.4.3 Magnetic Properties

I.5 THERMOPHYSICAL EFFECTS

1.6 CHEMICAL PROPERTIES .

1.7 NUCLEAR PROPERTIES

1.8 NOMENCLATURE

1.9 SERVICE LIMITS

$1.10 \cdot$ PROGESSING

1.10.1 Surface Treatment

1.10.2 Thermal Treatment

1.10.3 Sheet and Tubing

1.10.4 Joining

. $\quad 1.10 .5$ Forming

1.10.6 Machinability

2.0 MECHANICAL PROPERTIES

2.1 SHORT TERM

2.1.1 Static (Ultimate, Yield, etc.)

2.1.2 Dynamic (Strain rate, Impact)

2.2 LONG TERM (Creep, relaxation, etc.)

2.3 CYCLIC LOAD PROPERTIES (Fatigue) 


\begin{tabular}{|c|c|c|}
\hline \multirow[t]{2}{*}{3.0} & \multicolumn{2}{|c|}{ PHYSICAL PROPERTIES } \\
\hline & 3.1 & THERMAL PROPERTIES (Sp. Ht., thermal conductivity) \\
\hline & 3.2 & $\begin{array}{l}\text { ELECTRICAL/MAGNETIC PROPERTIES (Elec. resistivity, } \\
\text { magnetic resistivity, etc.) }\end{array}$ \\
\hline & 3.3 & MISCELLANEOUS (Viscosity, density) \\
\hline 4.0 & \multicolumn{2}{|c|}{ CHEMICAL PROPERTIES } \\
\hline 5.0 & \multicolumn{2}{|c|}{ NUCLEAR PROPERTIES } \\
\hline 6.0 & \multicolumn{2}{|c|}{ REFERENCES } \\
\hline
\end{tabular}




\subsection{BASIC DATA}

\subsection{GENERAL}

Types 304 and $304 \mathrm{~L}$ are the most commonly used of the austenitic stainless steels. 304L is the low carbon grade. These materials have high corrosion resistance, very good formability, and are readily weldrble by all techniques.

The properties given in section E are for AISI 304 and are average, except for tensile ultimate strength, tensile yield strength, elongation, and reduction in area. These latter properties are $80 \%$ of average.

\subsection{COMMERCIAL DESIGNATIONS}

Wrought: $\quad$ AISI 304, AISI $304 \mathrm{~L}$

Cast: $\quad C F-8, C F-3$

Available Forms, Specifications, Suppliers

\begin{tabular}{|l|l|}
\hline Forms and Conditions & Specifications \\
\hline $\begin{array}{l}\text { Sheet, Strip, and Plate } \\
\text { (Solution-Treated) }\end{array}$ & MIL-S-5059 \\
\hline $\begin{array}{l}\text { Tubing, Hydraulic } \\
\text { (Cold Drawn) }\end{array}$ & QQ-S-766, AMS 5513 \\
\hline $\begin{array}{l}\text { Tubing, Seamless and } \\
\text { Welded (Sol.- Treated) }\end{array}$ & MIL-T-6845, AMS 5566 \\
\hline $\begin{array}{l}\text { Bar Forgings, Tubing } \\
\text { (Solution-Treated) }\end{array}$ & AMS 5560, AMS 5565 \\
\hline Wire (Sol. Treated) & MIL-S-7720, QQ-S-763 \\
\hline $\begin{array}{l}\text { Sheet, Strip, and Plate } \\
\text { (304 L Sol. Treated) }\end{array}$ & AMS 5639 \\
\hline
\end{tabular}


1.3 CHEMICAL COMPOSITION (WT. \%)

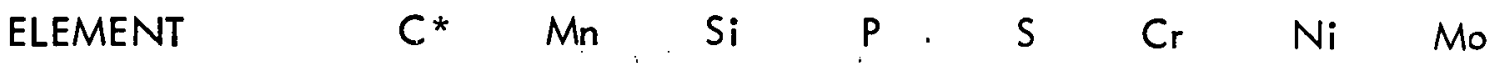

$\begin{array}{lllllllll}\text { MAXIMUM } & 0.08 & 2.00 & 1.00 & .040 & .030 & 20.0 & 11.0 & 0.50\end{array}$

MINIMUM $\quad{ }^{*}$ For 304 L, Max. C - - $18.00,8.00,-$

Content is $0.030 \%$. $\quad 0.50$ Bal':

I.4 : ROOM TEMPERATURE PROPERTIES

(Average Unless Otherwise Noted)

1.4.1 Mechanical Properties

$$
S_{\text {Sheet Strip }} \frac{\text { Annealed }}{\text { Plate, Bar }}
$$

Tensile Ultimate Strength

$\mathrm{F}_{\mathrm{TU}}$

PSI

$$
68,000 \cdot 68,000
$$

Tensile Yield Strength

$$
28,000
$$

F $\mathrm{TY}$

$$
24,000
$$

Comp. Yield Strength

$\mathrm{F} C Y$

PSI

Ultimate Shear Strength

$\mathrm{F}_{\mathrm{SU}}$

PSI

Shear Yield Strength

F

PSI

Ultimate Bearing Strength

$F_{B R U}$

PSI

$\mathrm{e} / \mathrm{D}=1.5$

$e / D=2.0$

Yield Bearing Strength

$F_{\text {BRY }}$

PSI

$\mathrm{e} / \mathrm{D}=1.5$

$\mathrm{e} / \mathrm{D}=2.0$

Elongation

e

$\%$ 
Reduction of Area

RA

$\%$

50

Modulus of Elasticity

E $\quad 10^{6}$ PSI

26

Comp. Modulus of Elasticity

$\mathrm{E} C \longrightarrow 10^{6} \mathrm{PSI}$

Modulus of Rigidity

G $\quad 10^{6} \mathrm{PSI}$

(Dynamic)

13

Poisson's Ratio

\subsection{5}

Hardness

$R_{B}$

Plastic Bendina Modulus

\subsubsection{Physical Properties}

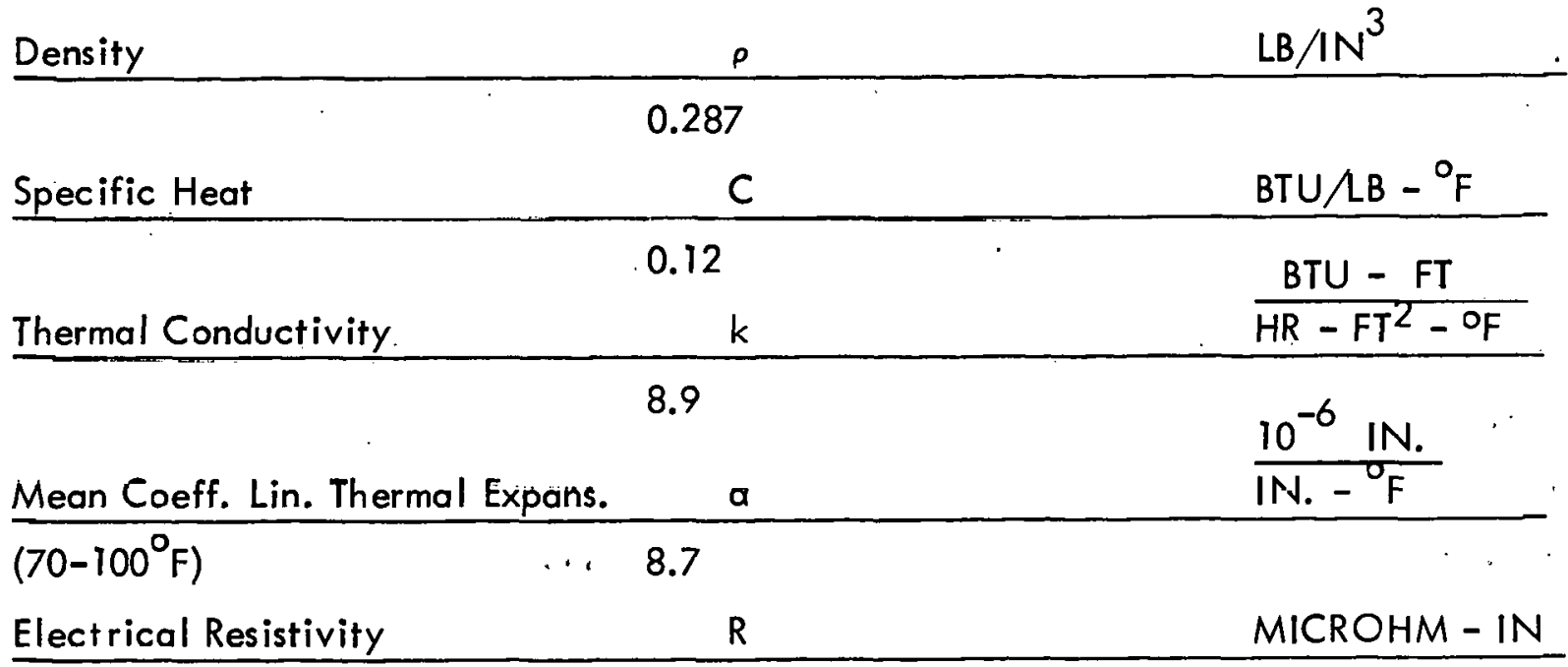

28.

\subsubsection{Magnetic Properties}

Non-magnetic in the annealed condition, but becomes slightly magnetic when cold worked.

1.5 THERMOPHYSICAL EFFECTS

Melting Range 
Phase Changes

Subject to carbide precipitation at $800^{\circ}-1600^{\circ} \mathrm{F}$.

Thermal Conductivity Vs. Temperature

\begin{tabular}{rc}
$\begin{array}{r}\text { Temperature } \\
\left.\rho_{F}\right)\end{array}$ & $k\left(\frac{B T U-F T}{H R-F T^{2}-{ }^{\circ} F}\right)$ \\
\hline-423 & 1.33 \\
-320 & 4.00 \\
-200 & 6.11 \\
0 & 8.05 \\
75 & 8.44 \\
200 & 8.95 \\
400 & 9.94 \\
600 & 10.95 \\
800 & 11.95 \\
1000 & 13.21 \\
1200 & 14.11 \\
1350 & 15.06
\end{tabular}

\subsection{CHEMICAL PROPERTIES}

General corrosion resistance to various atmospheres, most acids, steam and combustion gases, is very good.

Passivating is necessary to develop best corrosion resistance.

Oxidation resistance is good up to $1700^{\circ} \mathrm{F}$ for continuous service and to $1600^{\circ} \mathrm{F}$ for intermittent service.

Intergranular corrosion after welding or heating may occur in type 304. 


\section{I.7 NUCLEAR PROPERTIES}

Austenitic stainless steels retain their high impact strength after irradiation.

\subsection{NOMENCLATURE}

$\mathrm{R}$ In fatigue the algebraic ratio of the minimum stress to the maximum stress in one cycle, that is, R $\frac{S M I N}{S M A X}$

ELC Extra low carbon

L Low carbon grade

\subsection{SERVICE LIMITS}

Type $304 \mathrm{~L}$

$-423^{\circ} \mathrm{F}$ to $800^{\circ} \mathrm{F}$ (Not recommended above $800^{\circ} \mathrm{F}$ because of relatively low strength.

\subsection{PROCESSING}

\subsubsection{Surface Treatment}

Scale may be removed by a solution of $15-20$ percent nitric acid and $1-3$ percent hydrofluoric acid at $120^{\circ}$ to $140^{\circ} \mathrm{F}$, for 20 to 30 minutes. Scale is more readily removed when parts have been heated in air.

Chlorinated cleaners, such as trichloroethylene, should be avoided, as these may contribute to stress-corrosion cracking of the alloy.

\subsubsection{Thermal Treatment}

Furnace atmospheres should be neutral or slightly oxidizing. Heating with high sulfur fuels should be avoided.

Bright annealing is done in a dry hydrogen, cracked ammonia, or argon atmosphere with a dew point of $-80^{\circ} \mathrm{F}$ maximum. 


\subsubsection{Sheet and Tubing}

1900 to $1950^{\circ} \mathrm{F}, 10$ minutes hold at temperature, air cool up to 0.064 inch in thickness, water quench 0.065 inch and thicker.

\subsubsection{Joining}

Can be welded readily by all methods. Type 308 weld wire generally used.

\section{Welding:}

For parent metal welded to itself, select one of the following weld rods:
AISI 308/MIL-R-503I, CL. 1
AISI 308 ELC/MIL-R-503I, CL. 16
ER 308/ASTM-A-37I, CL. ER308
ER 308L/ASTM-A-37I, CL. ER308L

\subsubsection{Forming}

\section{Formability:}

Excellent formability in the annealed condition may require intermediate annealing in severe forming operations.

\section{Forging:}

Starting forging temperature $2300^{\circ} \mathrm{F}$ maximum, finishing temperature $1500^{\circ} \mathrm{F}$ minimum. Severe reduction below $1700^{\circ} \mathrm{F}$ should be avoided.

\section{Castability:}

Excellent.

\subsubsection{Machinability}

Machinability is about $35 \%$ of Bessemer Steel screw stock. 


\subsection{MECHANICAL PROPERTIES \\ (As Function of Temperature)}

\section{I SHORT TERM}

\subsubsection{Static}

Figure 2.1-1

Figure 2.1-2

Figure 2.1-3

Figure 2. 1-4

Figure 2.1-5

Figure 2.1-6

Figure 2. 1-7

Figure 2.1-8

Figure 2. 1-9

Figure 2.1-10

Figure 2.1-11

Figure 2. $1-12$

Figure 2.1-13

Figure 2.1-14
Ultimate Tensile Strength (Minimum Expected)

Ultimate Tensile Strength

Yield Strength (Minimum Expected)

Yield Strength

Total Elongation (Minimum Expected)

Uniform Elongation (Minimum Expected)

Total Elongation

Uniform Elongation

Hardness (Nominal)

Poisson's Ratio

Young's Modulus

Shear Modulus

Rupture Modulus

Rupture Modulus 


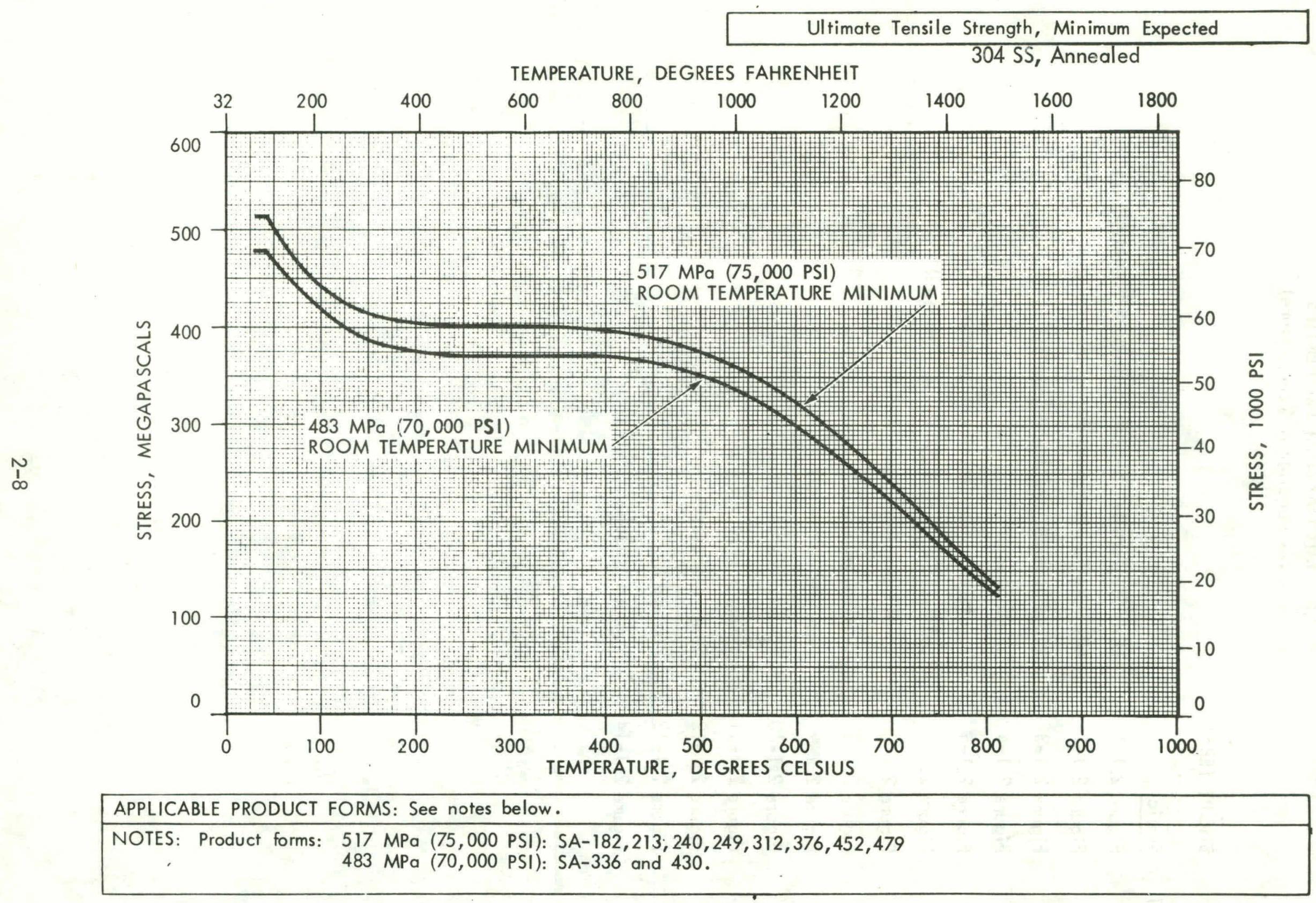

Figure 2.1-1 


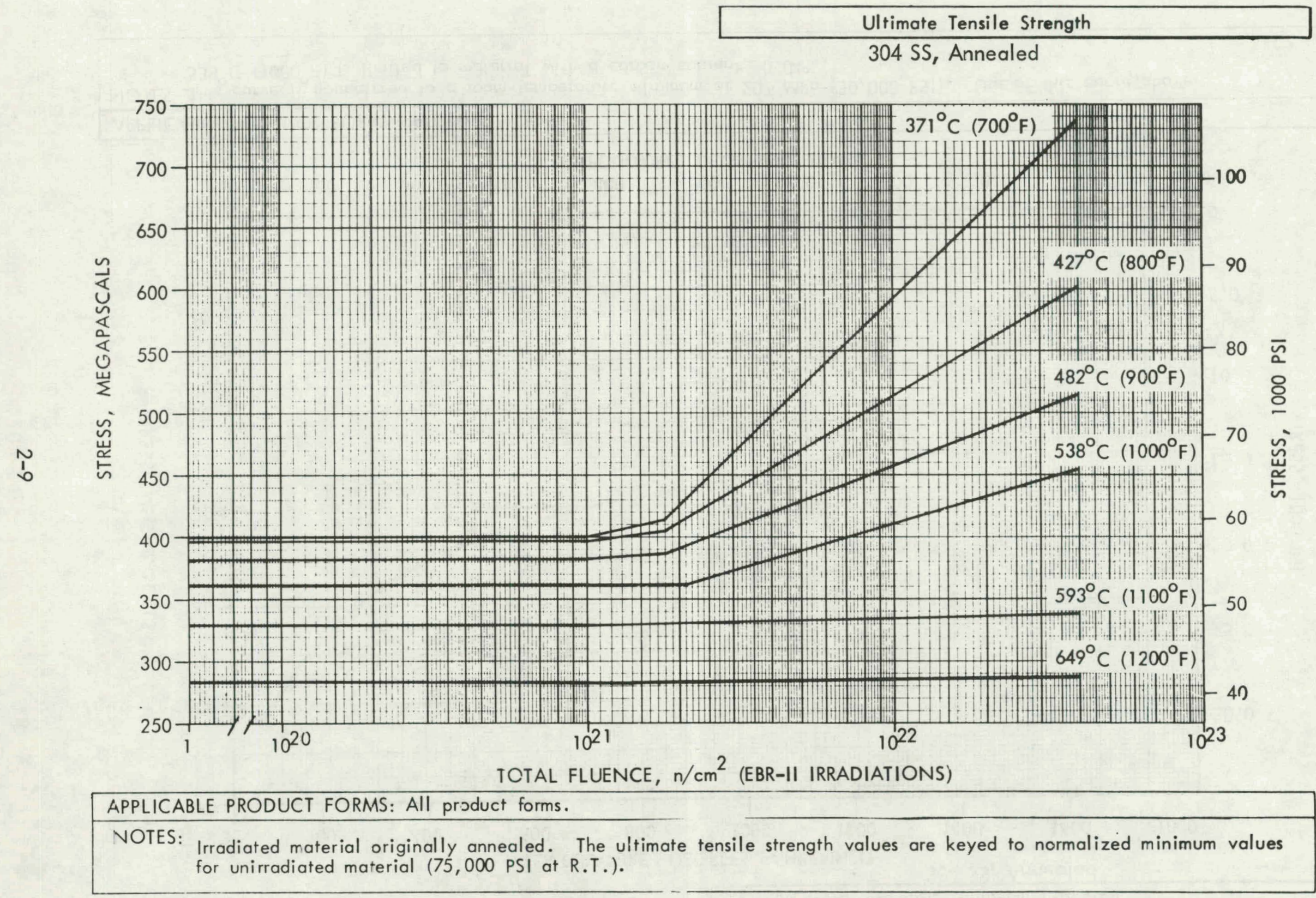

Figure 2.1-2 


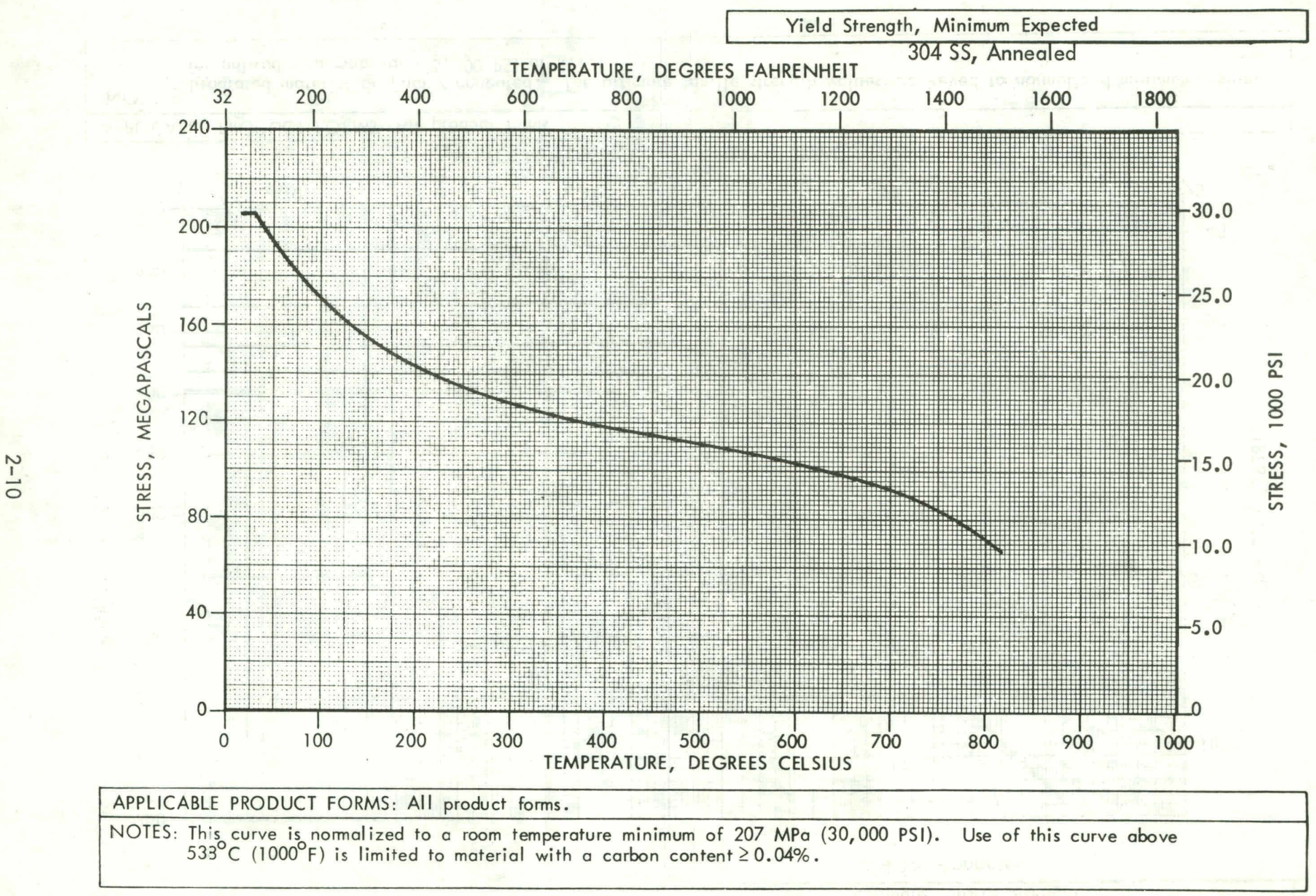

Figure 2.1-3 


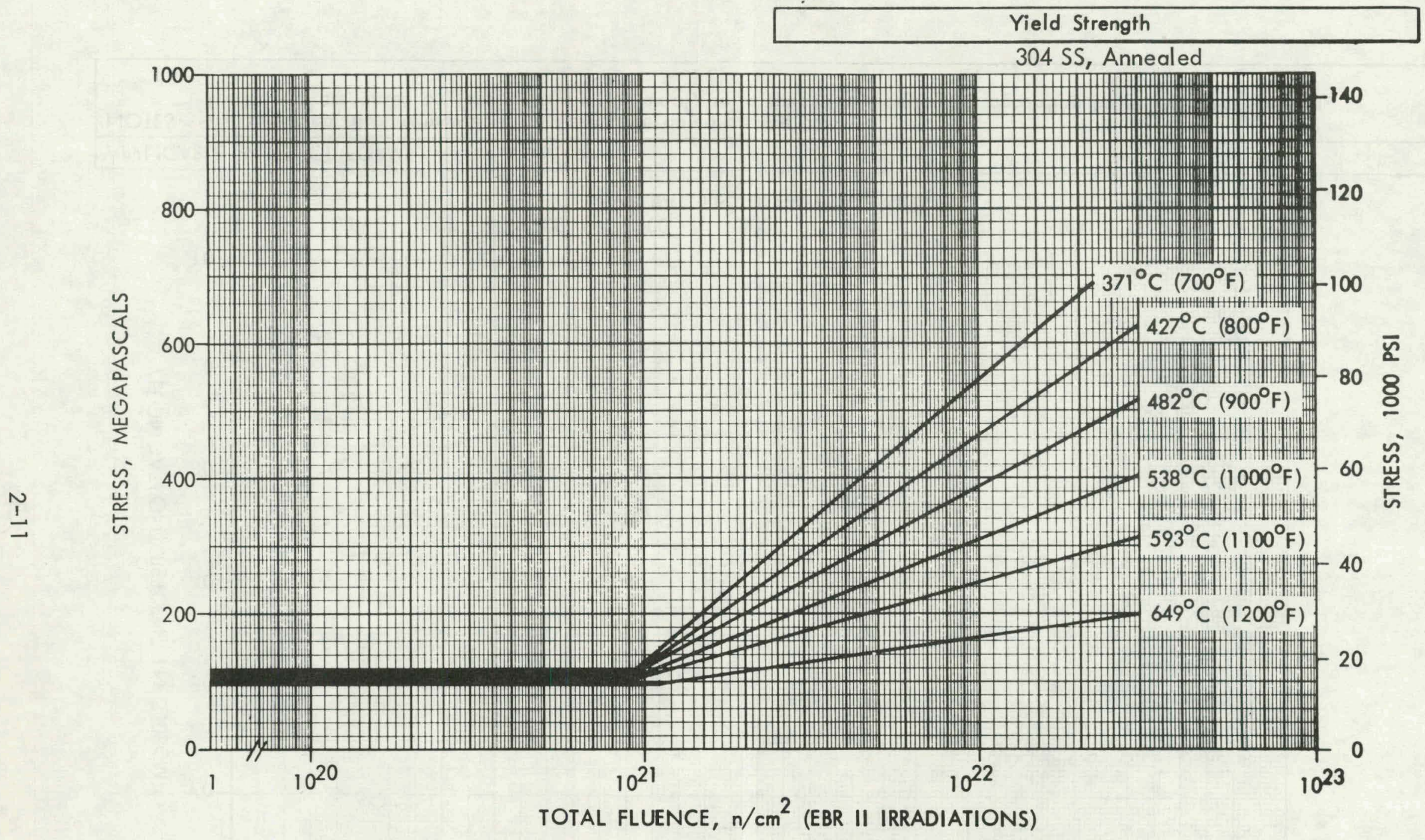

AFPLICABLE PRODUCT FORMS: All product forms.

NOTES: Irradiated material, originally annealed. $0.2 \%$ offset yield strength, keyed to normalized minimum values for unirradiated material.

Figure 2.1-4 


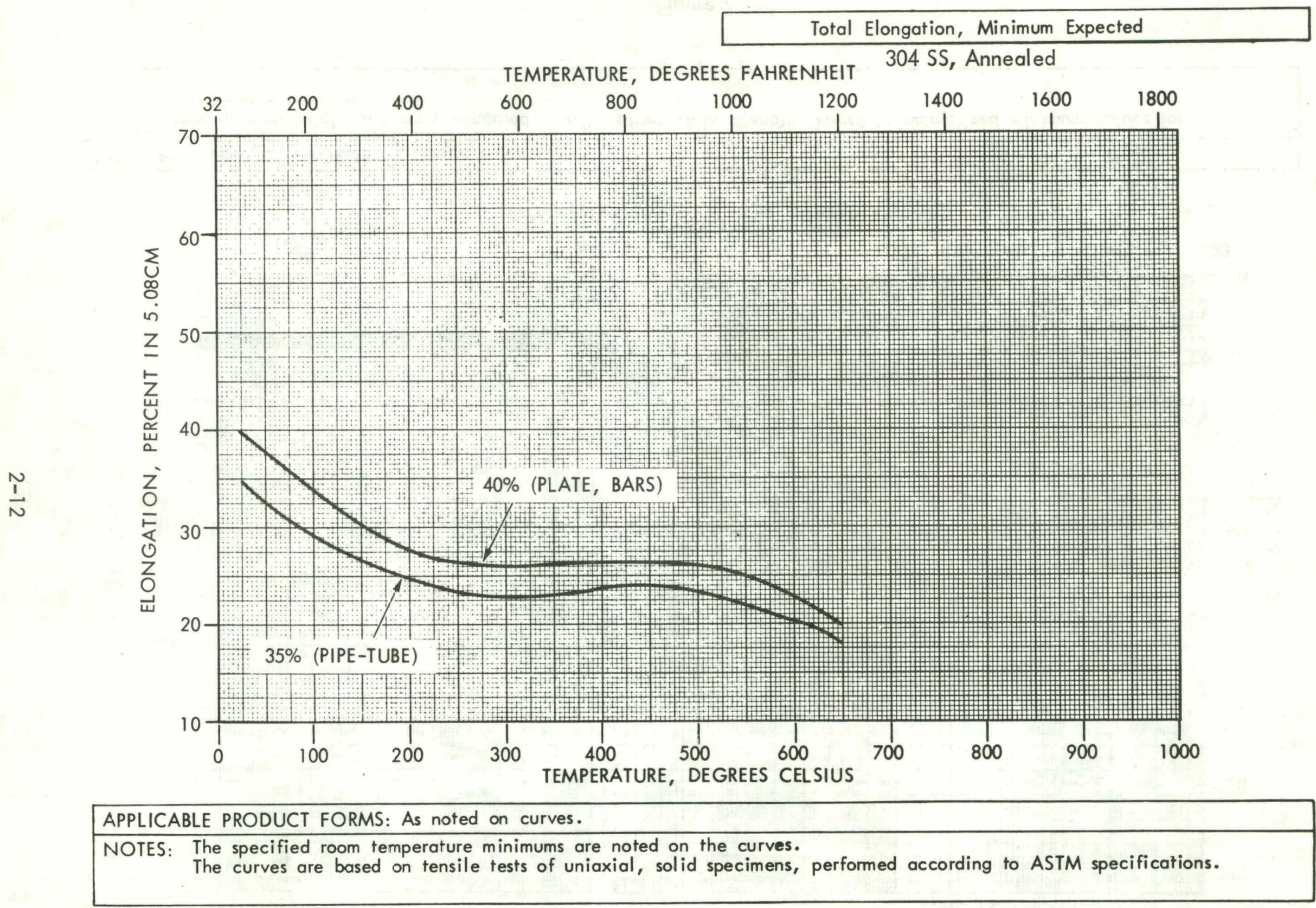

Figure 2.1-5 


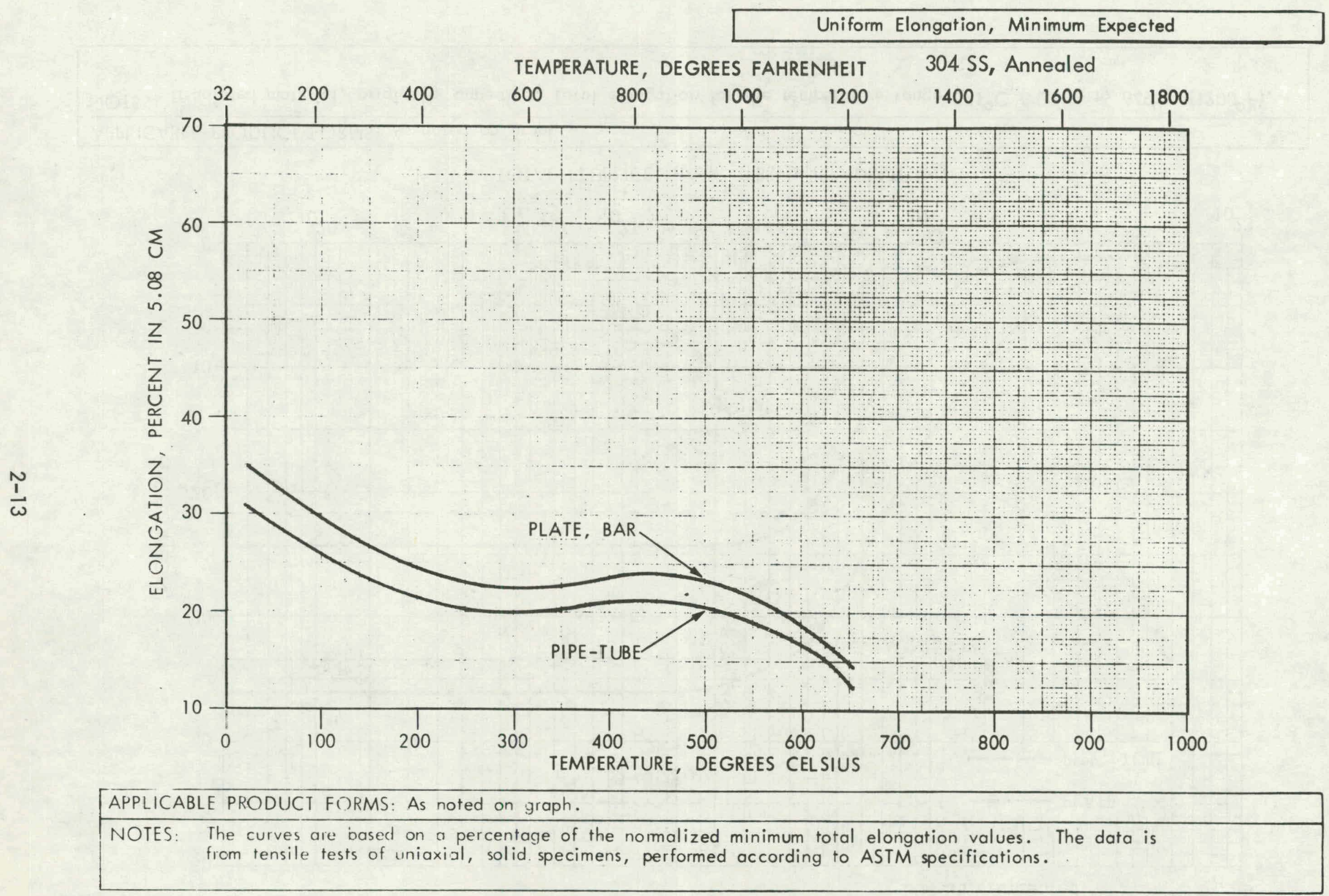

Figure 2.1-6 


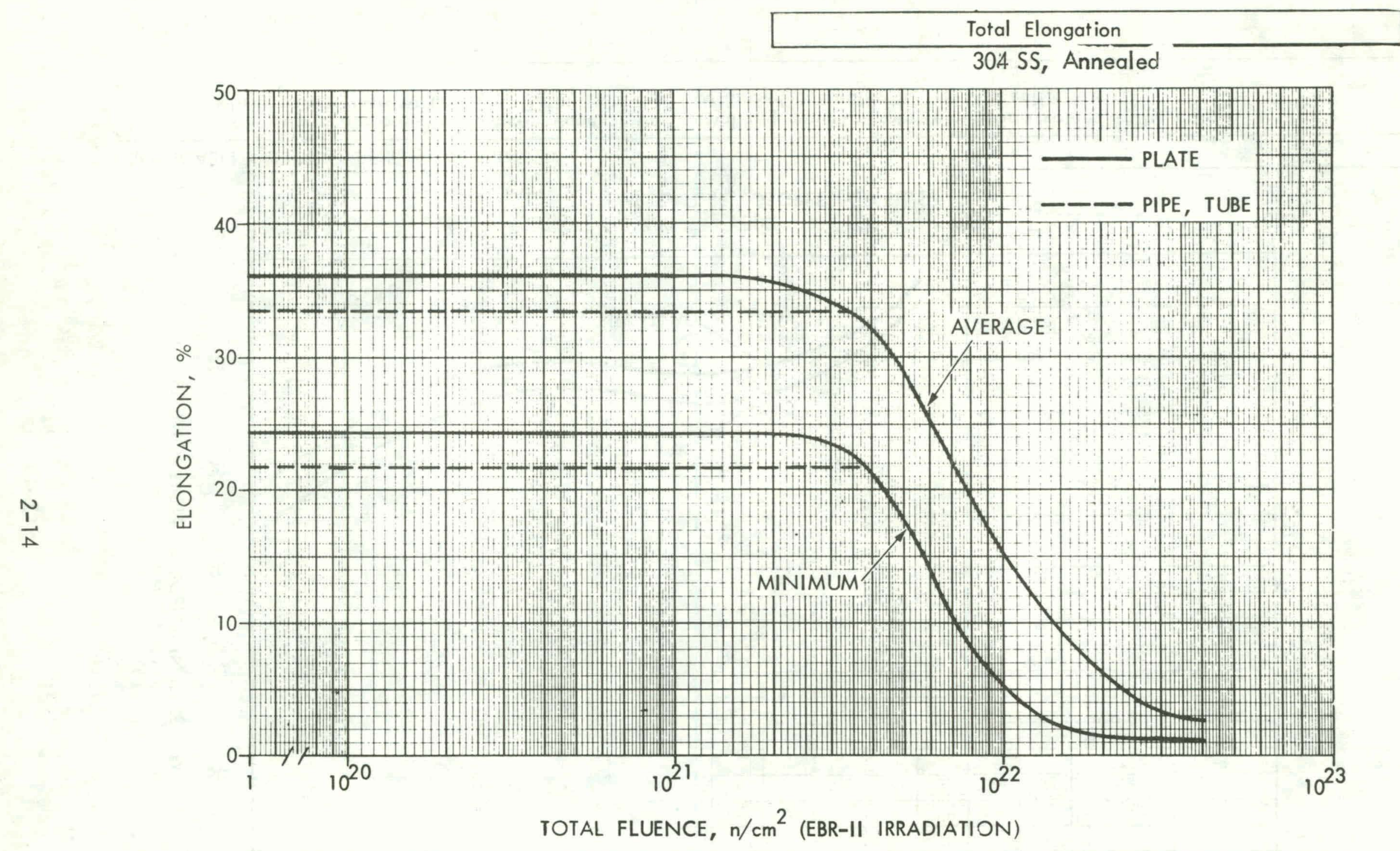

APPLICABLE PRODUCT FORMS: As noted on graph.

NOTES: Irradiated material, originally annealed. Total elongation for the temperature range $371^{\circ} \mathrm{C}\left(700^{\circ} \mathrm{F}\right)$ to $649^{\circ} \mathrm{C}\left(1200^{\circ} \mathrm{F}\right)$. 


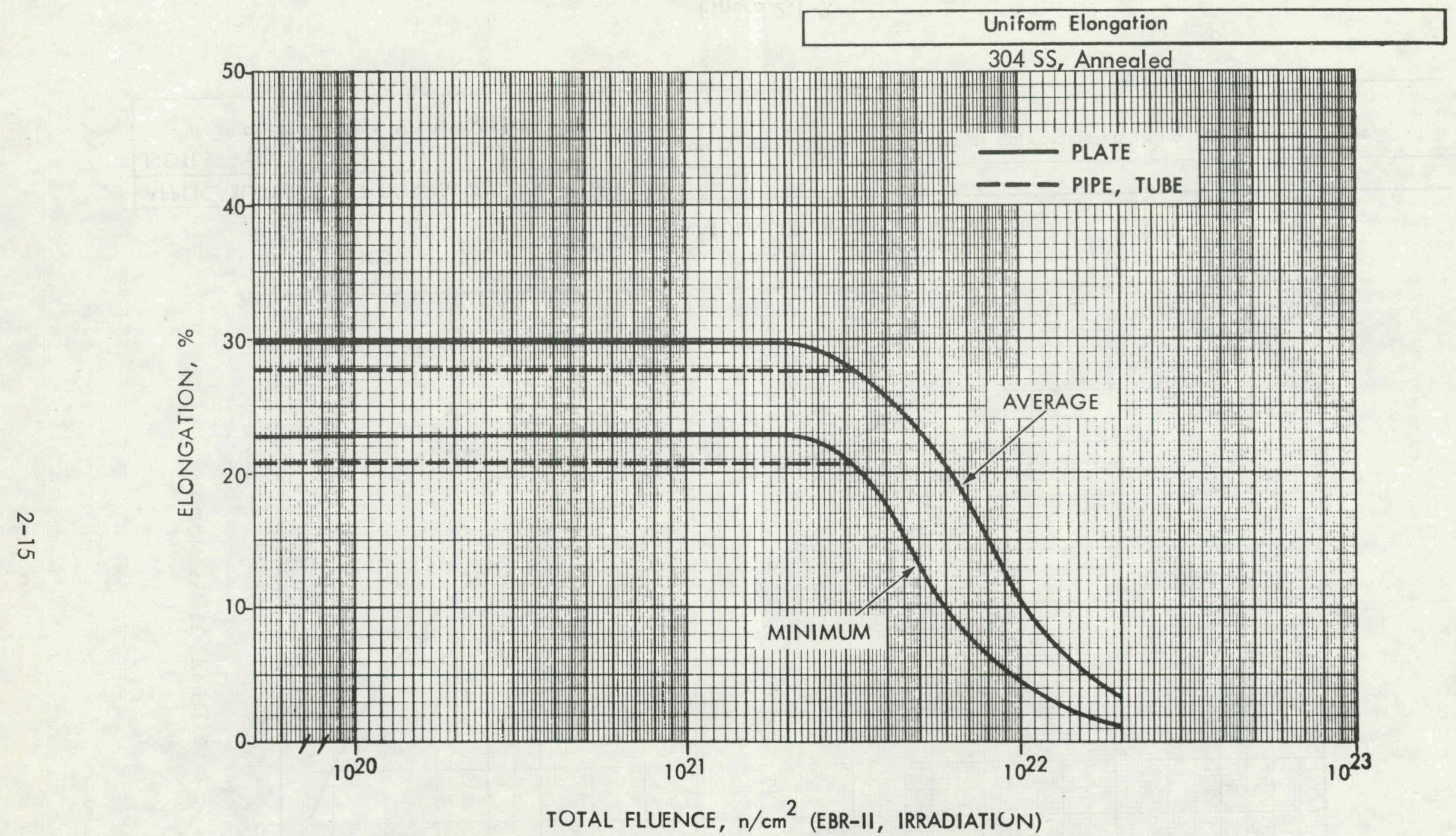

APPLICABLE PRODUCT FORMS: As noted on graph.

NOTES: Irradiated material, originally annealed. Total elongation for the temperature range $371^{\circ} \mathrm{C}\left(700^{\circ} \mathrm{F}\right)$ to $649^{\circ} \mathrm{C}\left(1200^{\circ} \mathrm{F}\right)$.

Figure 2.1-8 


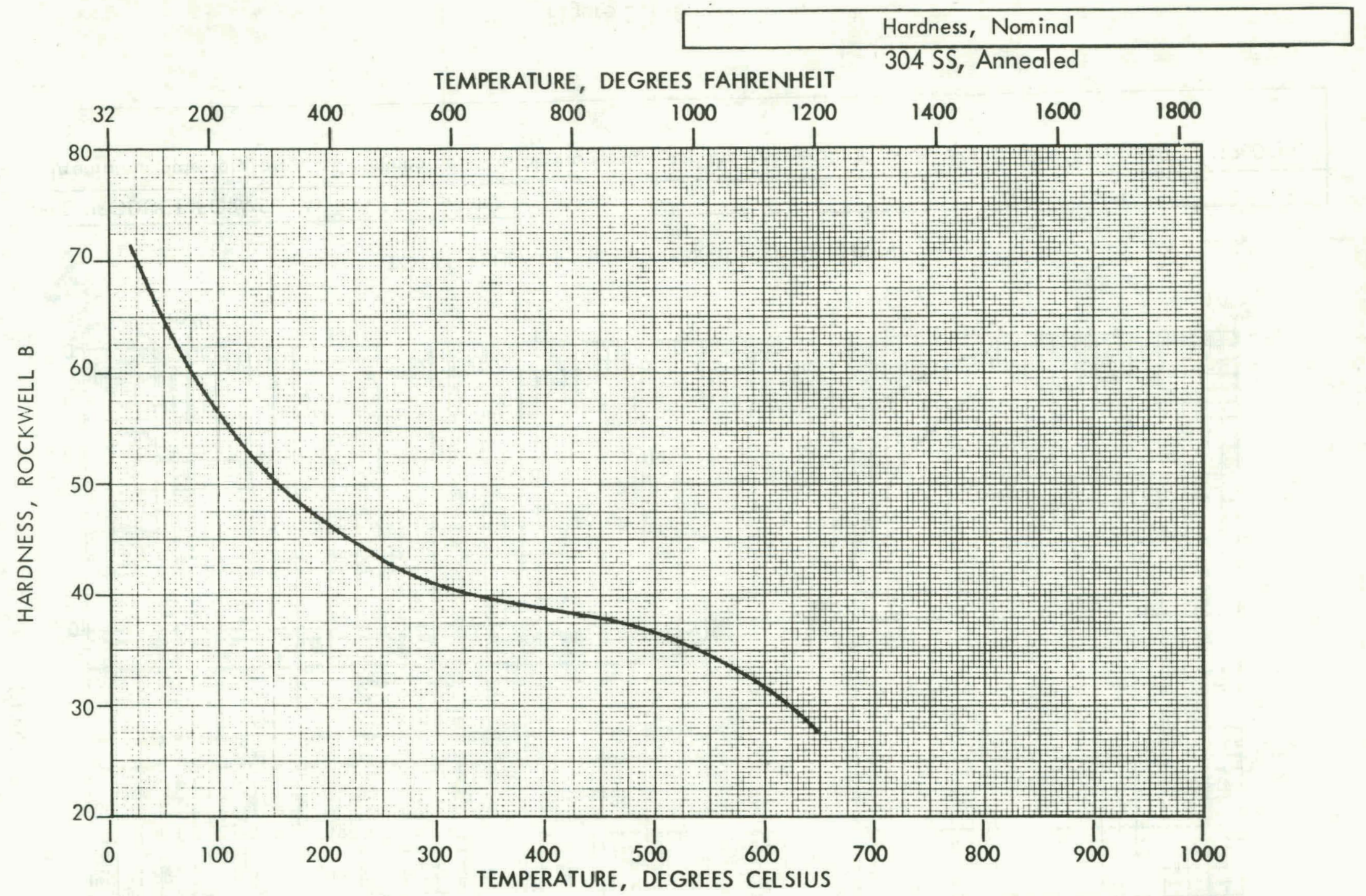

APPLICABLE PRODUCT FORMS: All product forms.

NOTES: The shape of the curve between room temperature and $538^{\circ} \mathrm{C}\left(1000^{\circ} \mathrm{F}\right)$ was approximated using the general shape of an ultimate strength curve.

Figure 2.1-9 


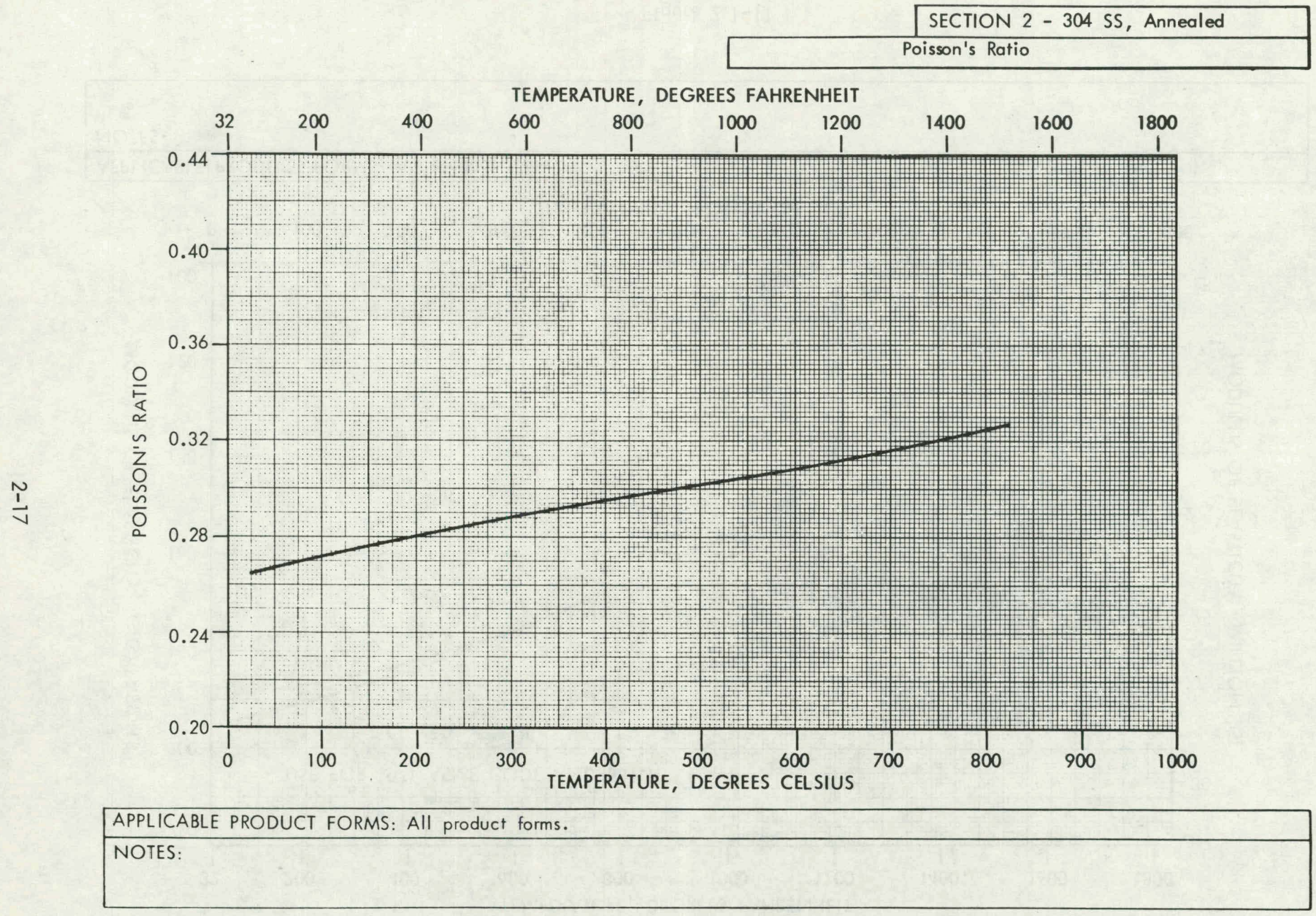

Figure 2.1-10 
SECTION 2 - 304 SS, Annealed

Young's Modulus, Static

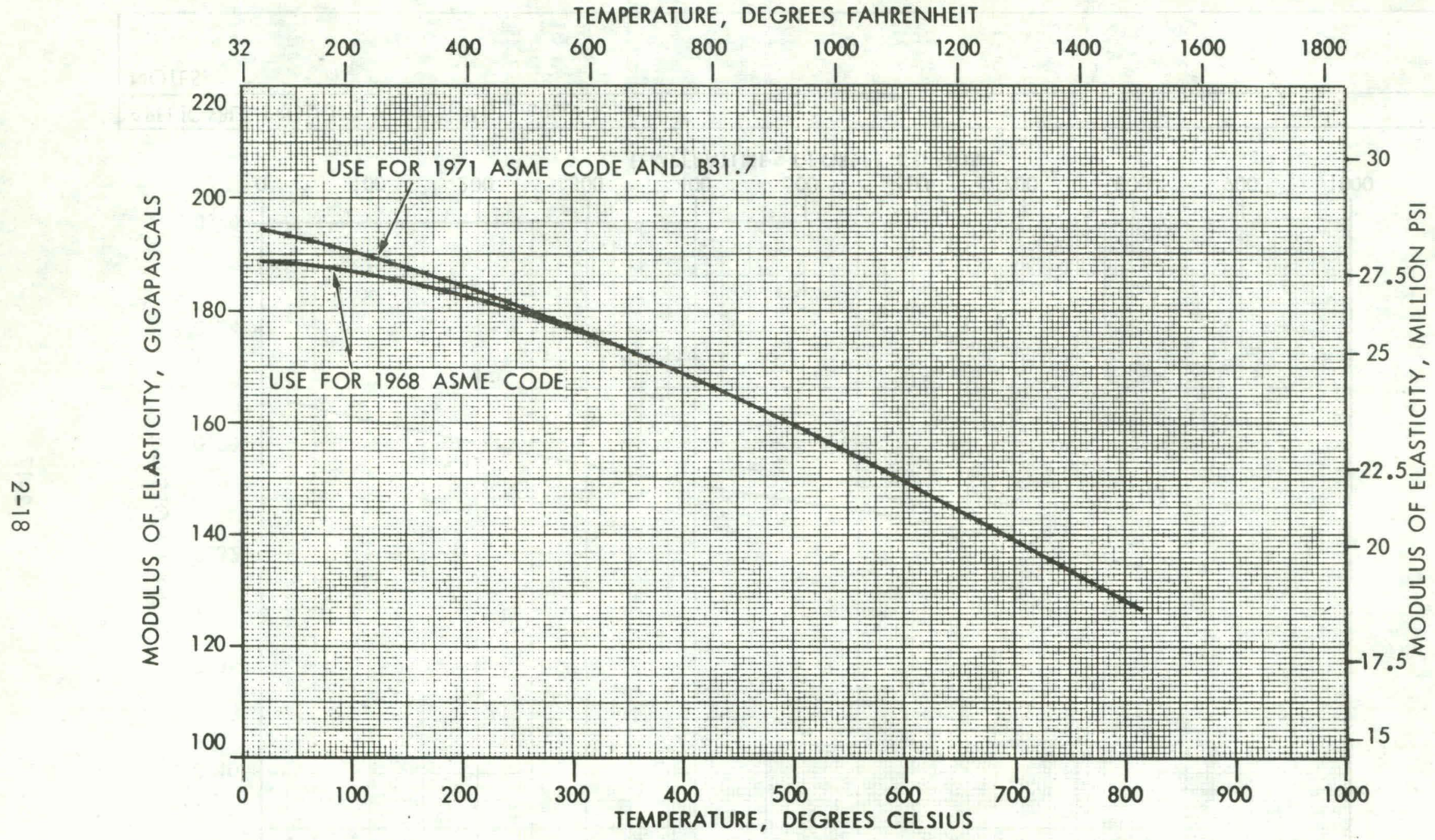

APPLICABLE PRODUCT FORMS: All product forms.

NOTES:

Figure 2.1-11 


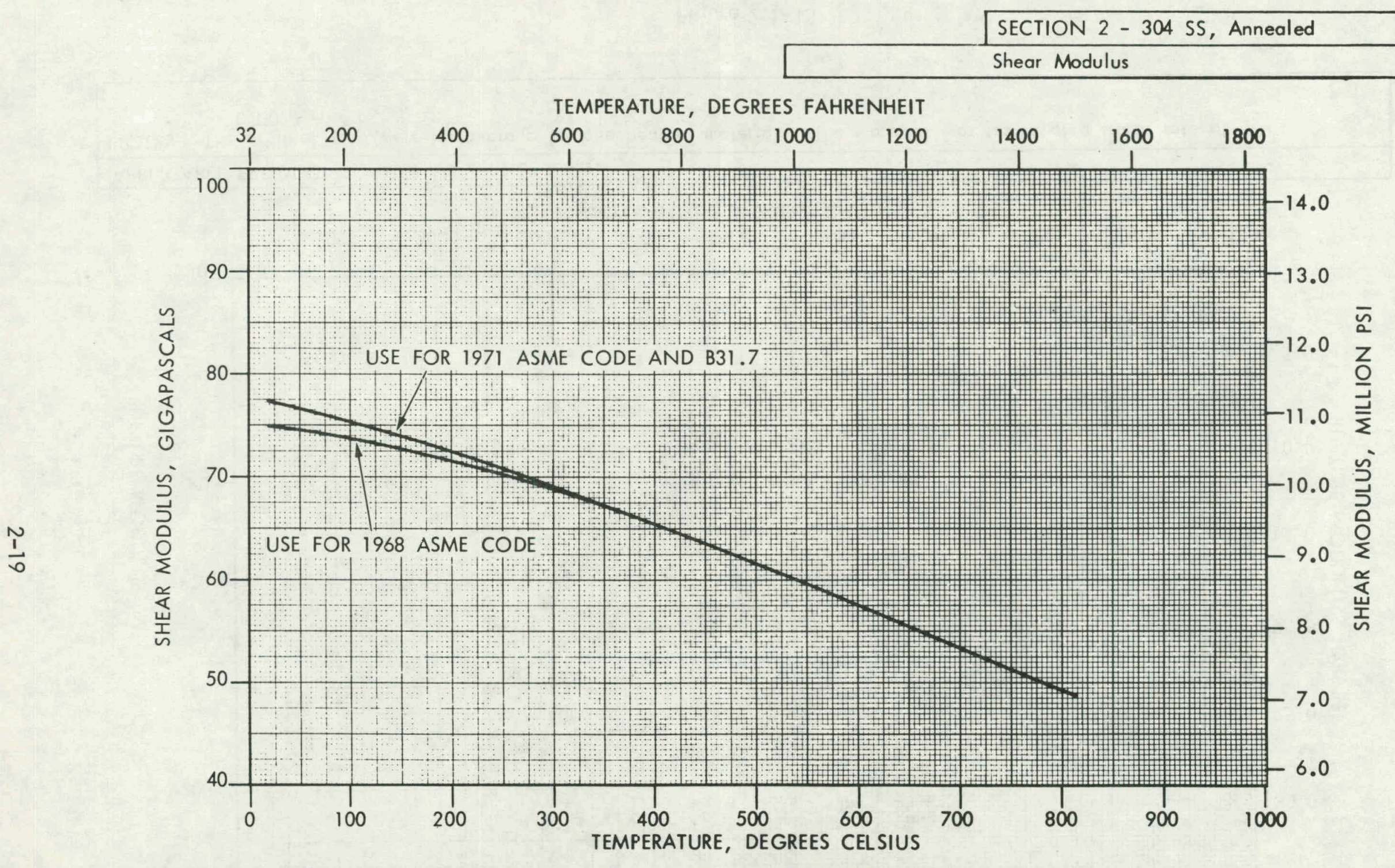

APPLICABLE PRODUCT FORMS: All product forms.

NOTES: These curves were calculated from the equation: $E /(2(1+\nu))$

Figure 2.1-11 
SECTION 2 - 304 SS, Annealed

Rupture Modulus

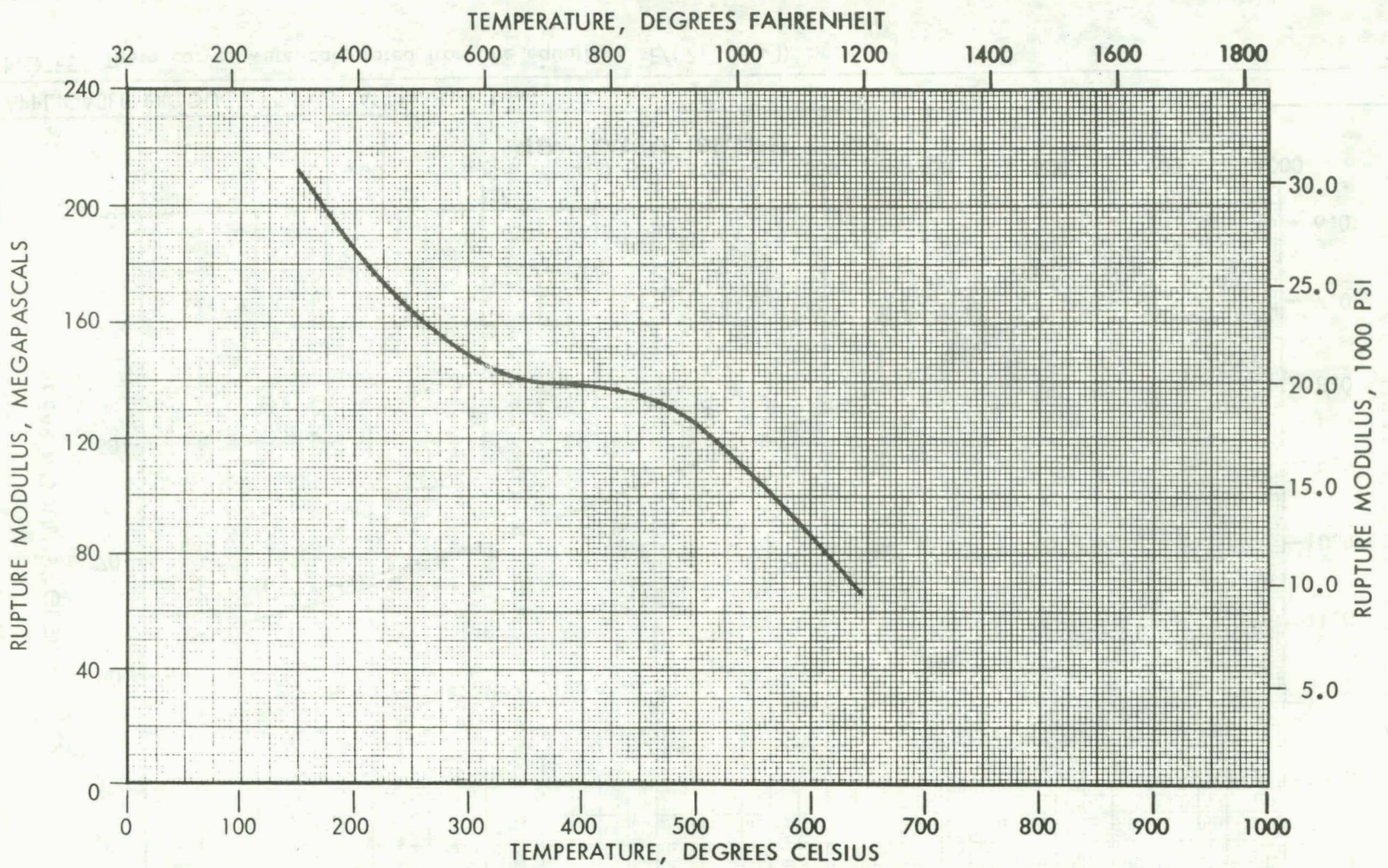

APPLICABLE PRODUCT FORMS: All product forms.

NOTES: The original data were an average for one heat of material. The material was tested at a strain rate of $\sim 0.0022 \mathrm{~min}^{-1}$.

Figure 2.1-13 
SECTION 2 - 304 SS, Annealed

Rupture Modulus

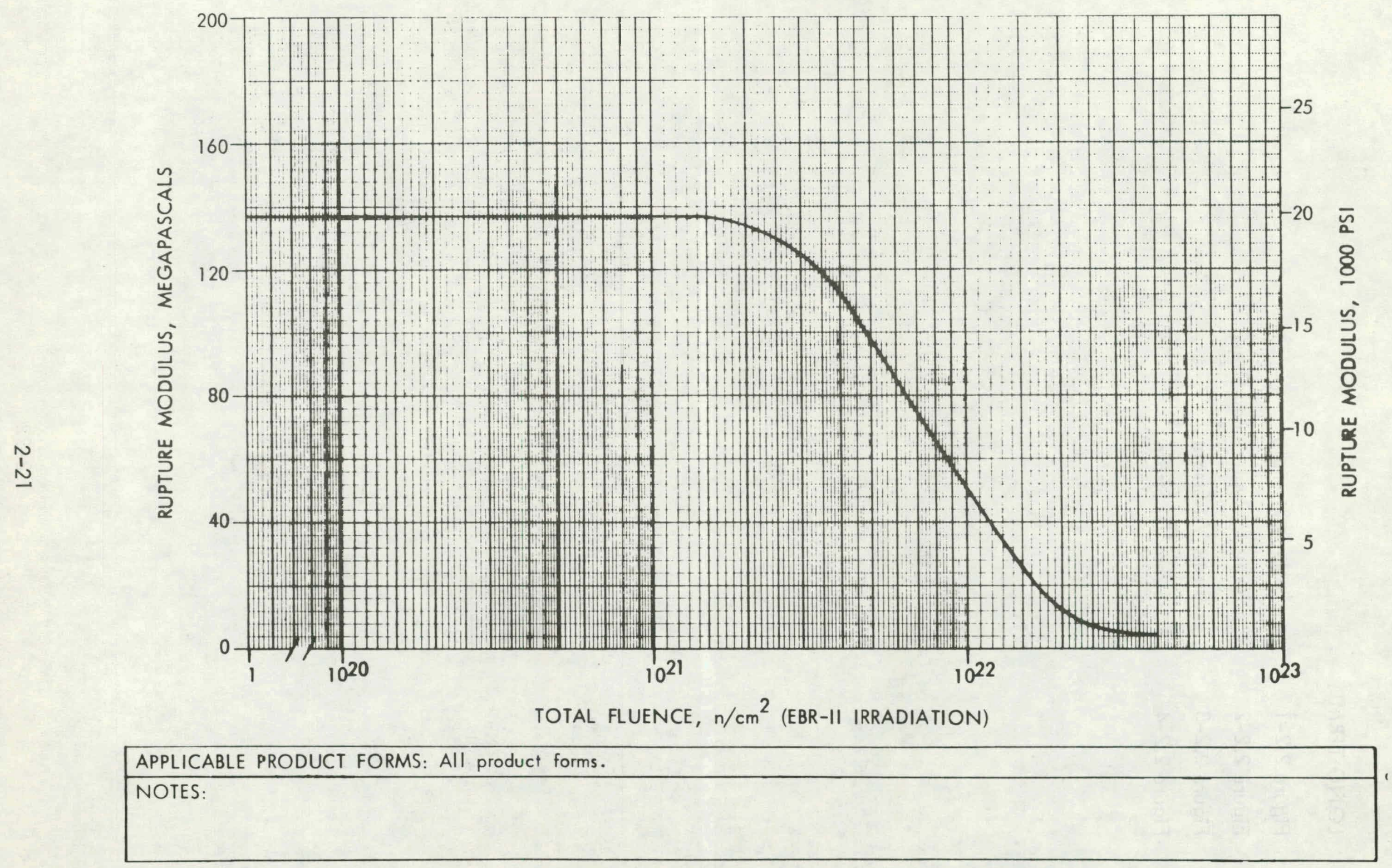

Figure 2.1-14 
2.2 LONG TERM

Figure 2.2-1

Figure 2.2-2

Figure 2.2-3

Figure 2.2-4
Fatigue Strength

Stress to Rupture and Creep

Stress Vs. Strain

Stress Vs. Strain 

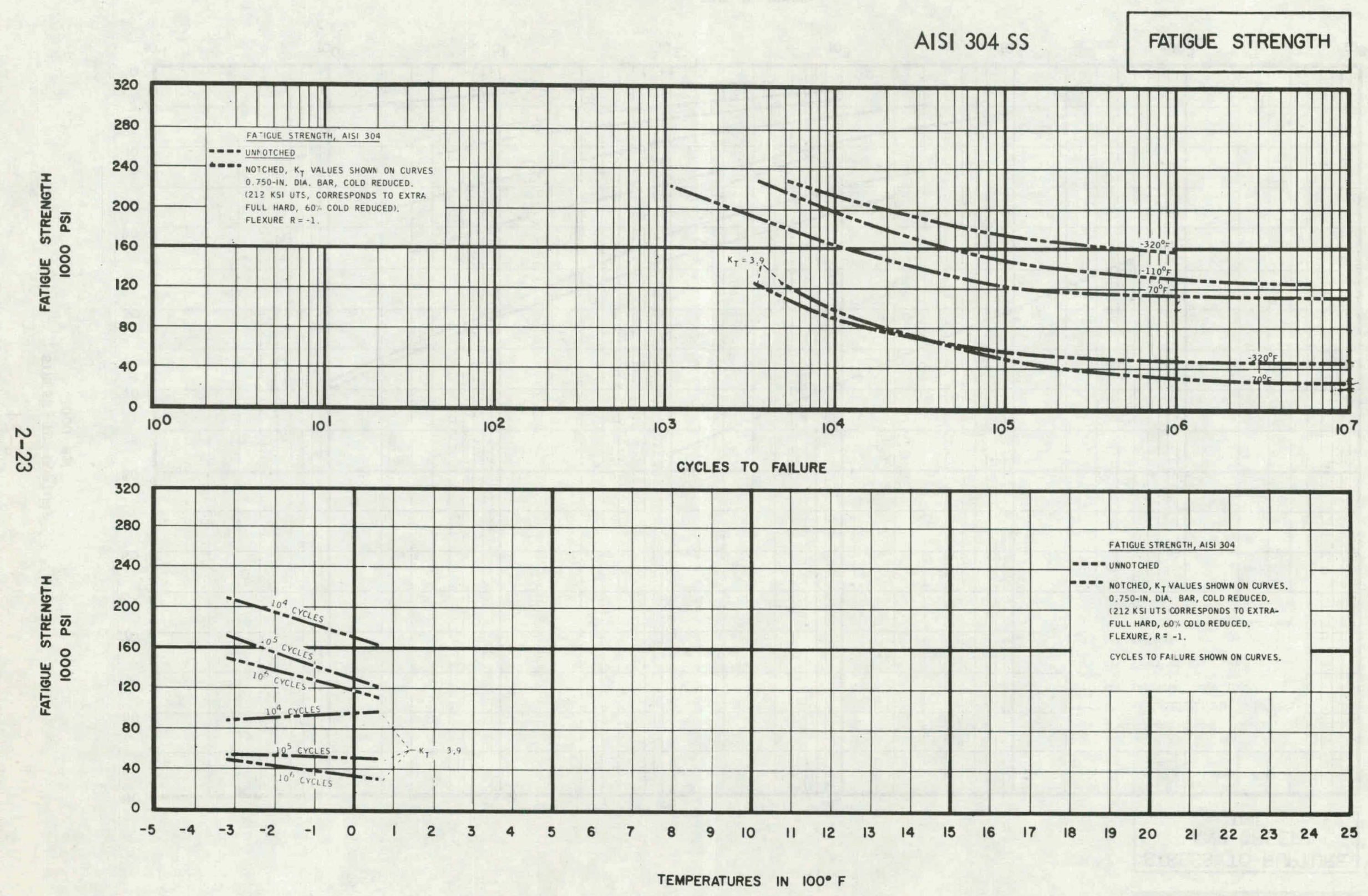

Figure 2.2-1 


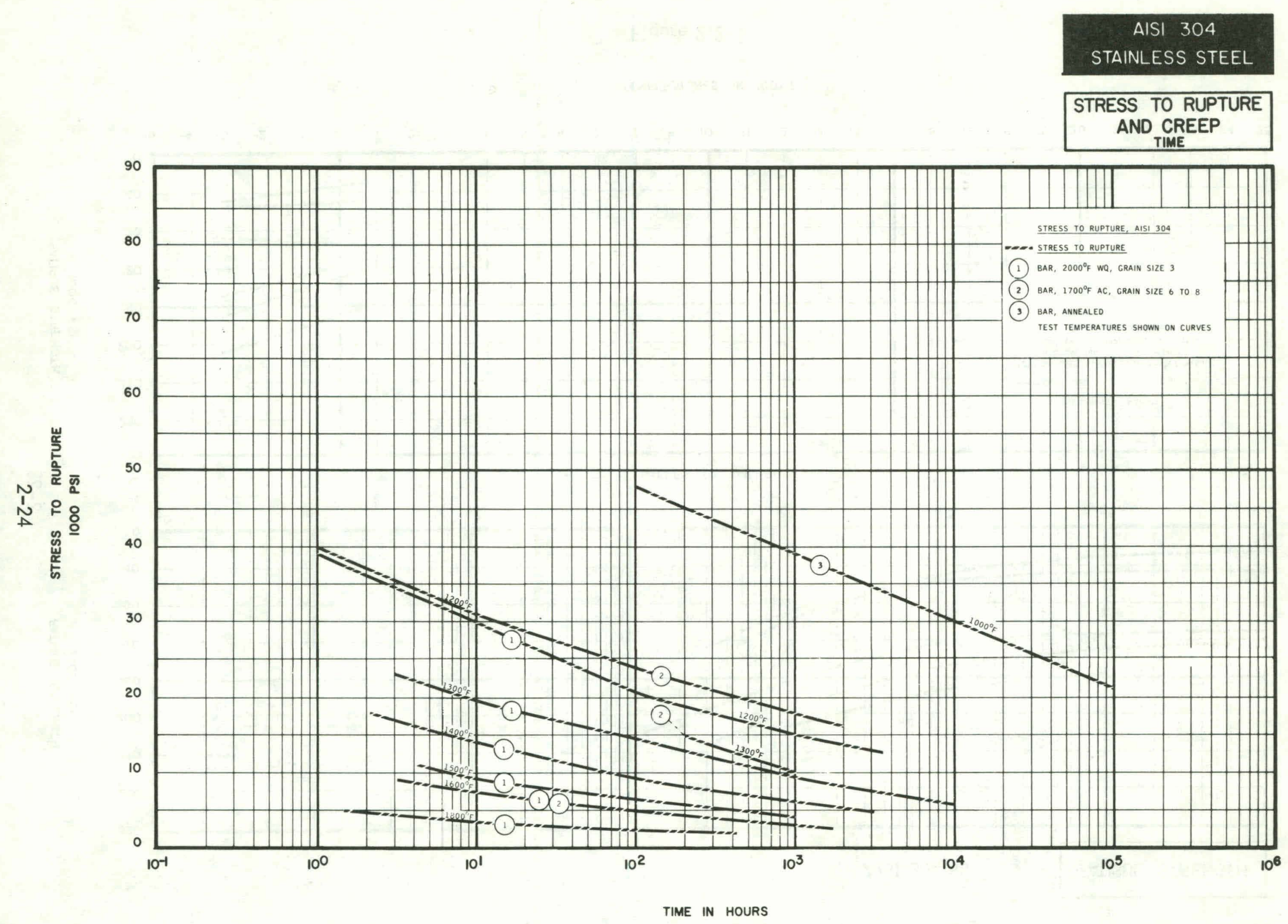

Figure 2.2-2 


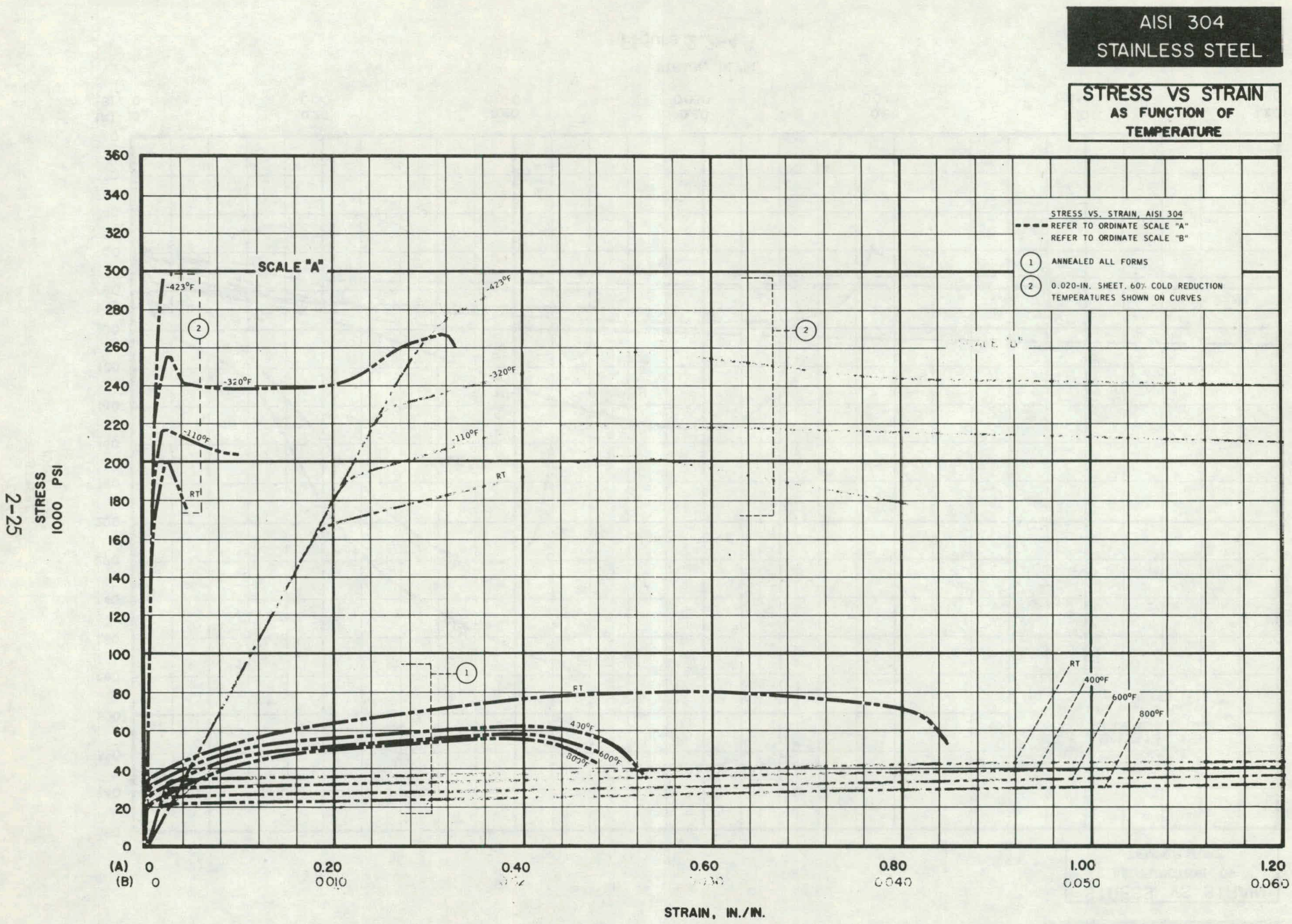

Figure 2.2-3 
AISI 304

STAINLESS STEEL

STRESS VS STRAIN

AS FUNCTION OF

TEMPERATURE

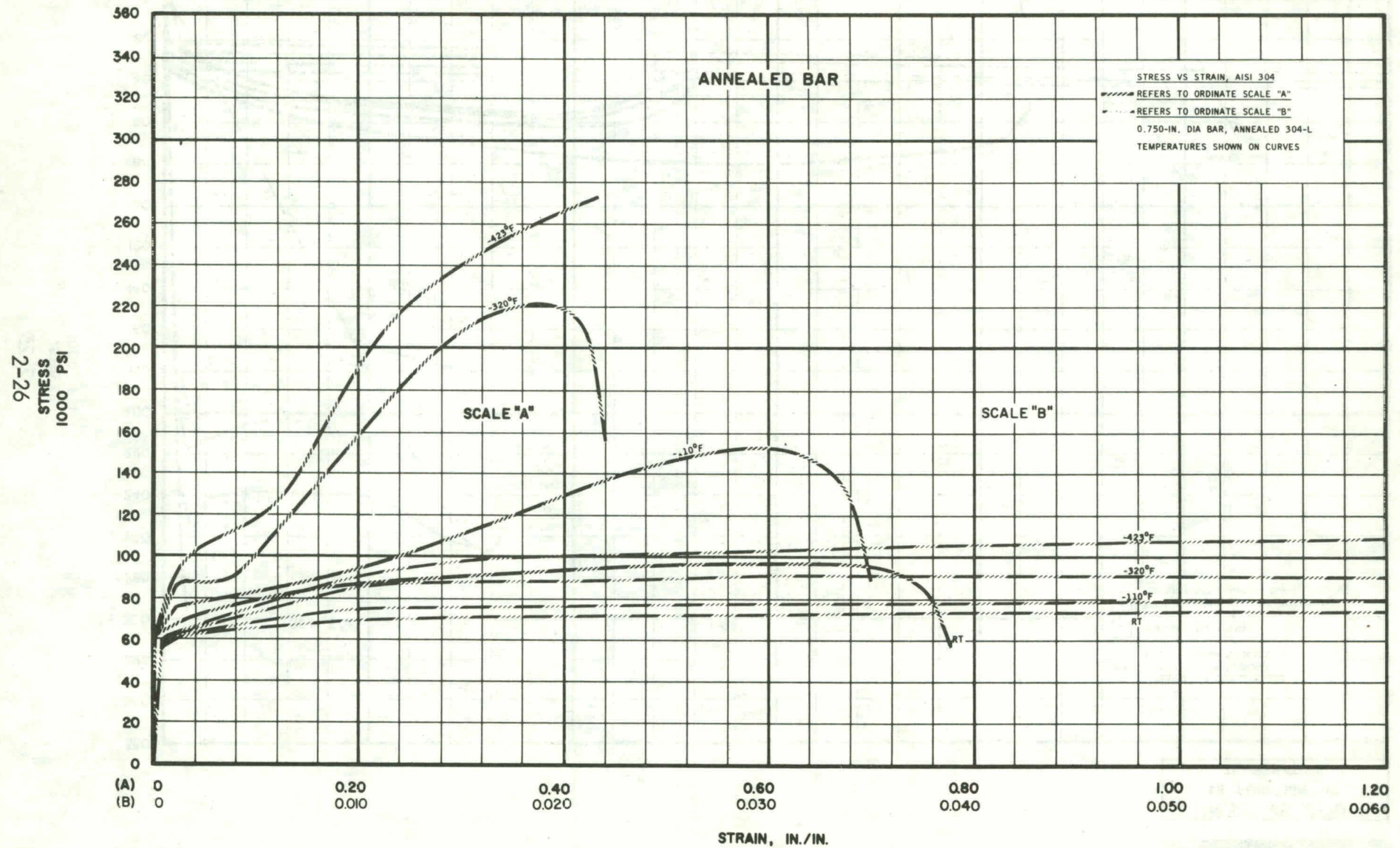

Figure 2.2-4 


\subsection{PHYSICAL PROPERTIES}

3.1 THERMAL PROPERTIES

Figure 3.1-1 Thermal Expansion/Specific Heat 
THERMAL EXPANSION VALUES ARE FROM

THERMAL EXPANSION

ROOM TEMPERATURE TO INDICATED TEMP.

SPECIFIC HEAT
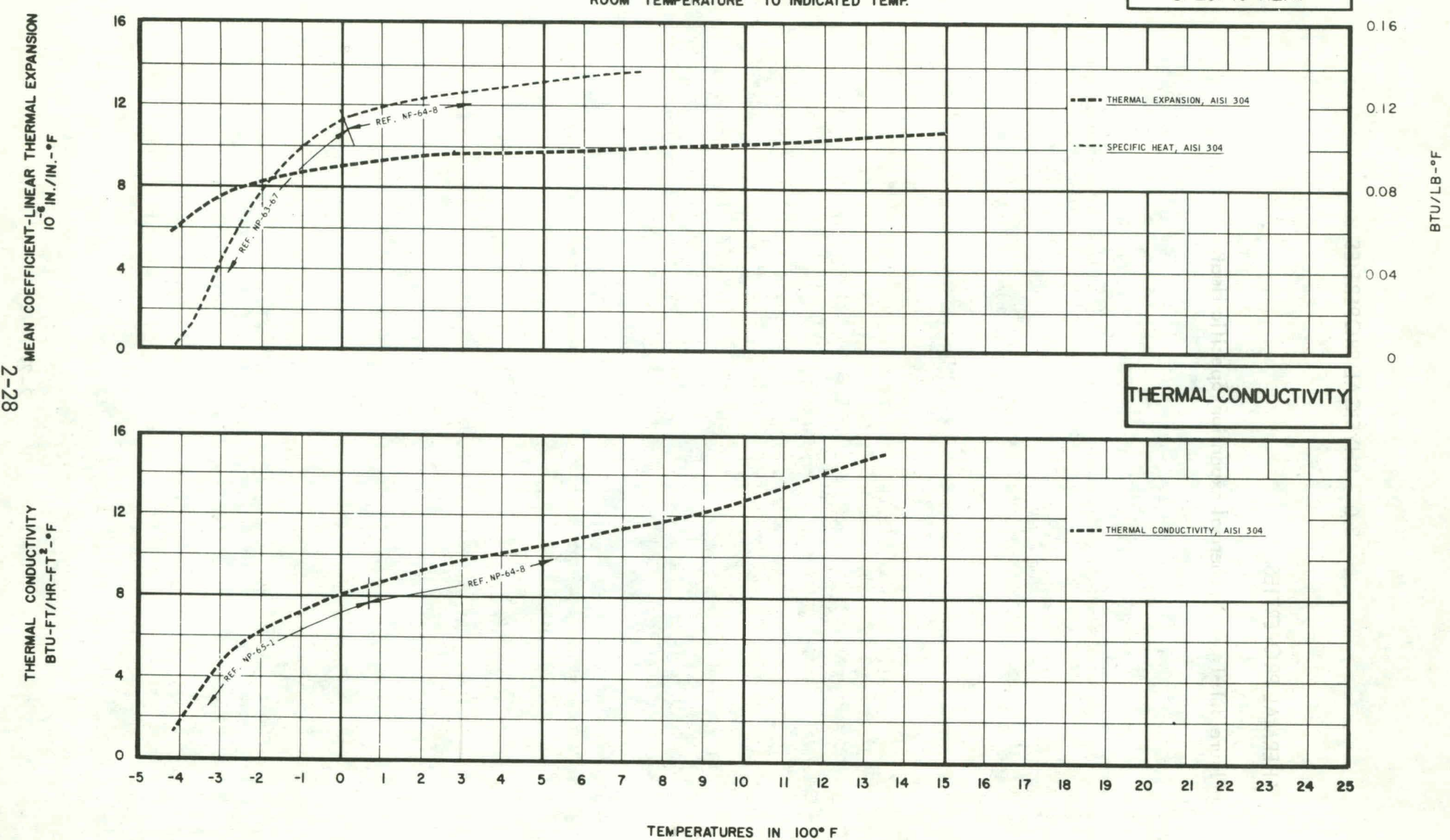

Figure 3.1-1 
4.0 CHEMICAL PROPERTIIES 
5.0 NUCLEAR PROPERTIES 


\subsection{REFERENCES}

6.1 Aerospace Structural Metals Handbook.

6.2 Simmons, W. F. and Cross, H. C., "The Elevated Temperature Properties of Stainless Steels," ASTM STP-124, 1952.

6.3 Simmons, W. F. and Van Echo, T. A., "The Elevated Temperature Properties of Stainless Steels," ASTM DS5-S1, 1965.

6.4 Cryogenic Materials Data Book, ML-TDR-64-280 (PB 171809, Revised) AFSC, Wright-Patterson AFB, Aug 1964, with Supplements through July 1965.

6.5 Low Temperature Data Sheet, Types 304 and 304 L Stainless Steels, INCO, March 1963. 
SECTION 3.0

TYPE 305 STAINLESS STEEL 


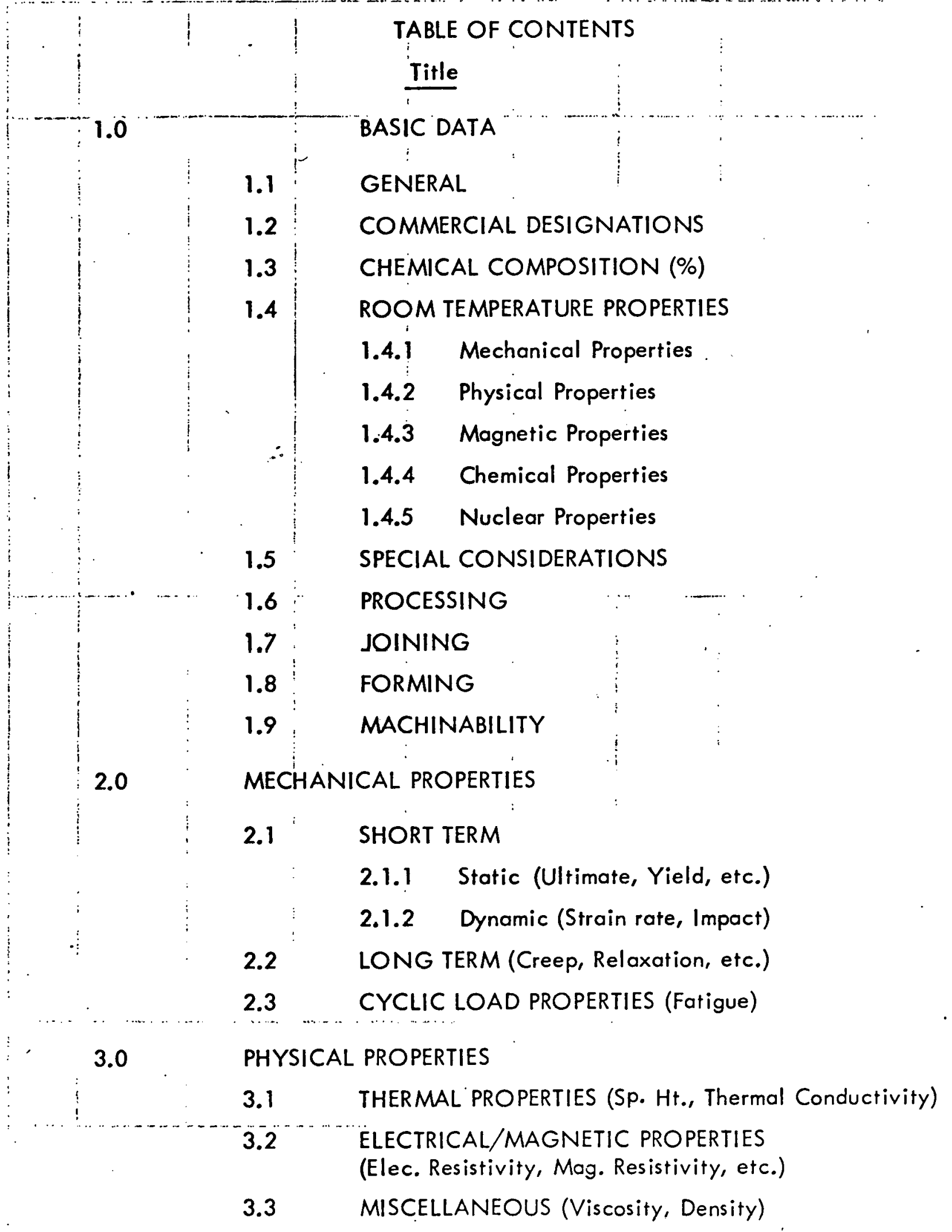


4.0

CHEMICAL PROPERTIES

5.0

NUCLEAR PROPERTIES

6.0

REFERENCES 


\subsection{BASIC DATA}

\subsection{GENERAL}

Type 305 austenitic stainless steel is the member of the $18 \mathrm{Cr}-8 \mathrm{Ni}$ steel family having the highest nickel content considered to be with in this classification. It is, therefore, the austenitic stainless steel with the lowest rate of strain hardening. It is used in sheet, strip and plate form for difficult drowing, spinning and other forming operations and in wire form for severe upsetting. It also becomes less magnetic on cold work than other 18-8 steels, particularly Type 301. This steel has a higher carbon content than Type 304 and, therefore, a greater susceptibility to intergranular corrosion after exposure at 800 to $1500 \mathrm{~F}$. The properties of this alloy are otherwise nearly identical to those of Type 304 .

\subsection{COMMERCIAL DESIGNATIONS}

AISI Type 305 austenitic stainless steel, 18-8 FS stainless steel, 18-12 steel, 30305.

Available Forme, Specifications

\begin{tabular}{|l|c|c|}
\hline \multicolumn{1}{|c|}{ Form } & AMS & ASTM \\
\hline $\begin{array}{l}\text { Sheet, strip and plate } \\
\text { Bar and billet }\end{array}$ & $5514 \mathrm{~A}$ & $\mathrm{~A}-167, \mathrm{~A}-240$ \\
Tube & & $\mathrm{A}-276, \mathrm{~A}-314$ \\
$\begin{array}{l}\text { Forging } \\
\text { Wire; rope and safety } \\
\text { Wire, riveting and cold } \\
\text { heading }\end{array}$ & $5685 \mathrm{D}$ & $\mathrm{A}-473$ \\
$\begin{array}{l}\text { Wire, cold Finished or } \\
\text { cold drown rounds } \\
\text { and shapes }\end{array}$ & $5686 \mathrm{~A}-492$ \\
Wire, weaving & & $\mathrm{A}-493$ \\
& & $\mathrm{~A}-580$ \\
\hline
\end{tabular}

This steel is available in plate, sheet, strip, bar, shapes billet, forging and wires. Plate, sheet, strip, bar, shapes and wires may be obtained in hot finished and annealed, cold finished and annealed and cold worked conditions. 


\subsection{CHEMICAL COMPOSITION (\%)}

\begin{tabular}{|c|c|c|c|c|c|c|}
\hline Source & & & & 6.10 & $\overline{\operatorname{Ref}}$ & \\
\hline Alloy & \multicolumn{6}{|c|}{$\mathrm{Fe}-18 \mathrm{Cr}-12 \mathrm{Ni}$ (Type 305} \\
\hline Form & \multicolumn{2}{|c|}{ Plate, sheet, Strip } & \multicolumn{2}{|c|}{ Wire, safety } & \multirow{2}{*}{\multicolumn{2}{|c|}{ Wire, riveting }} \\
\hline & \multicolumn{2}{|c|}{\begin{tabular}{|l} 
Percent \\
\end{tabular}} & \multicolumn{2}{|c|}{ Percent } & & \\
\hline & $\min$. & $\max$. & $\min :$ & $\max$. & $\min$. & $\max$. \\
\hline \multirow{10}{*}{$\begin{array}{l}\text { Carbon } \\
\text { Manganese } \\
\text { Phosphorous } \\
\text { Sulfur } \\
\text { Silicon } \\
\text { Chromium } \\
\text { Nickel } \\
\text { Copper } \\
\text { Molybdenum } \\
\text { Iron }\end{array}$} & - & 0.12 & - & 0.08 & - & 0.08 \\
\hline & - & 2.00 & - & 2.00 & - & 2.00 \\
\hline & - & 0.040 & - & 0.040 & - & 0.040 \\
\hline & - & 0.030 & - & 0.030 & - & 0.030 \\
\hline & - & 1.00 & - & 1.00 & - & 1.00 \\
\hline & 17.00 & 19.00 & 17.00 & 19.00 & 17.00 & 19.00 \\
\hline & 10.00 & 13.00 & 10.00 & 13.00 & 10.00 & 13.00 \\
\hline & - & 0.50 & - & 0.50 & - & 0.50 \\
\hline & & 0.50 & & 0.50 & - & 0.50 \\
\hline & \multicolumn{2}{|c|}{ Balance } & \multicolumn{2}{|c|}{ Balance } & \multicolumn{2}{|c|}{ Balance } \\
\hline
\end{tabular}

\begin{tabular}{|c|c|c|c|c|}
\hline Source & \multicolumn{4}{|c|}{ Ref. 6.12 to 6.21} \\
\hline Alloy & \multicolumn{4}{|c|}{$\mathrm{Fe}-18 \mathrm{Cr}-12 \mathrm{Ni}$ (Type 305) } \\
\hline Form & \multicolumn{2}{|c|}{$\begin{array}{l}\text { Sheet, strip and plate: } \\
\text { bar and billet; tube; } \\
\text { forging; and wire }\end{array}$} & \multicolumn{2}{|c|}{$\begin{array}{l}\text { Bar and wire for cold } \\
\text { heading and cold } \\
\text { forging }\end{array}$} \\
\hline \multirow{10}{*}{$\begin{array}{l}\text { Carbon } \\
\text { Manganese } \\
\text { Phosphorous } \\
\text { Sulfur } \\
\text { Silicon } \\
\text { Chromium } \\
\text { Nickel } \\
\text { Iron }\end{array}$} & \multicolumn{2}{|c|}{ Percent } & \multicolumn{2}{|c|}{ Percent } \\
\hline & minimum & maximum & minimum & maximum \\
\hline & - & 0.12 & - & .0 .12 \\
\hline & - & 2.00 & - & 2.00 \\
\hline & - & 0.045 & - & 0.045 \\
\hline & - & 0.030 & - & 0.030 \\
\hline & - & 1.00 & - & 1.00 \\
\hline & 17.00 & 19.00 & 17.00 & 19.00 \\
\hline & 10.00 & 13.00 & 11.00 & 13.00 \\
\hline & \multicolumn{2}{|c|}{ Balance } & \multicolumn{2}{|c|}{ Balance } \\
\hline
\end{tabular}


1.4 ROOM TEMPERATURE PROPERTIES

1.4.1 Mechanical Properties

Tensile ultimate strength annealed

FTU psi

\begin{tabular}{|c|c|c|c|}
\hline annealed & 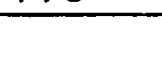 & 85,000 & \\
\hline$\frac{\text { Tensile yield strength }}{\text { annealed }}$ & FTY & 36,500 & psi \\
\hline Compressive yield strength & FCY & psi & \\
\hline Sheer strength & FSU & psi & \\
\hline Ultimate bearing strength & FBRU & psi & \\
\hline Elongation & e & $\%$ & \\
\hline Reduction of Area & RA & $\%$ & \\
\hline Modules of Elasticity & E & $10^{6} \mathrm{psi}$ & \\
\hline $28^{(2)}$ & & & \\
\hline Modules of Rigidity & $10^{6} \mathrm{psi}$ & & \\
\hline Compressive Modules of Elastic ity & & Ec & $10^{6} \mathrm{psi}$ \\
\hline Hardness & & RB & \\
\hline
\end{tabular}




\subsubsection{Physical Properties}

\begin{tabular}{llll} 
Density & $\rho$ & \multicolumn{1}{c}{$\mathrm{lb} / \mathrm{in}^{3}$} \\
\hline & 0.29 & \\
Specific Heat & ${ }^{\mathrm{c}}$ & $\mathrm{BTU} / \mathrm{lb}{ }^{\circ} \mathrm{F}$ \\
\hline & 0.12
\end{tabular}

$\frac{\text { Thermal Conductivity }}{\mathrm{BTU} / \mathrm{hr} / \mathrm{F} / \mathrm{st}}$

Coefficient of Thermal Expansion $\gamma \quad{ }_{F}{ }^{-1}$

$$
\begin{aligned}
& 9.6 \times 10^{-6}\left(32-212^{\circ} \mathrm{F}\right) \\
& 9.3 \times 10^{-6} \cdot\left(32-572^{\circ} \mathrm{F}\right)
\end{aligned}
$$

$\frac{\text { Electrical Resistivity } \quad \Omega \quad \text { Microhm-cm }}{72^{(2)}}$

\subsubsection{Magnetic Properties}

This steel is non-magnetic in the annealed condition. It becomes slightly magnetic when severely cold worked as a result of transformation of austenite to ferrite (martensite)(this effect is less pronounced than for the other 18-8 grades).

\subsubsection{Chemical Properties}

Excellent atmosphere resistance. General corrosion resistance of this steel is similar to that of Type 304.

This steel is only slightly embrittled by high pressure $(10,000 \mathrm{psi})$ gaseous hydrogen environment.

\subsubsection{Nuclear Properties}

The effect of neutron irradiation on room temperature tensile properties of annealed bar is given in the following table: 


\begin{tabular}{|l|c|c|}
\hline Source & \multicolumn{2}{|c|}{ Ref, 6.4 } \\
\hline Alloy & Fe-18 CR-12 Ni (Type 305) \\
\hline Form & \multicolumn{2}{|c|}{ Bar } \\
\hline Condition & \multicolumn{2}{|c|}{ Annealed } \\
\hline & Unirradiated & Irradiated* \\
\cline { 2 - 3 } $\mathrm{F}_{\text {tu }}-\mathrm{ksi}$ & 98.0 & 103.6 \\
$\mathrm{~F}_{\text {ty }}-\mathrm{ksi}$ & 32.1 & 71.4 \\
\hline *Irradiation $-3.9 \times 10^{19} \mathrm{n} / \mathrm{cm}^{2}$ of $80 \mathrm{C}$ \\
\hline
\end{tabular}




\subsection{SPECIAL CONSIDERATIONS}

When chromium-nickel steels containing more than about $0.04 \mathrm{C}$ are subjected to annealing at temperatures of 1850 to $2050 \mathrm{~F}$ and are then reheated to or held at temperatures of approximately 800 to 1600F, chromium carbides will precipitate at the austenite boundaries (Ref. 6.5, pg. 24). This intergranular precipitation or sensitization promotes intergranular corrosion cracking and reduces corrosion resistance of these steels. Heat treatment and welding conditions should be chosen to ovoid carbide formation. Additions of columbium and titanium are used to stabilize these alloys and reduce intergranular corrosion sensitivity.

\subsection{PROCESSING}

\section{Surface Treatment}

See ASTM A 380-57 entitled "Recommended Practice/or Descaling and Cleaning Stainless Steel Surfaces."

Thermal Treatment

Anneal or solution heat treat. 1850 to $2000 \mathrm{~F}, 1 / 2$ to 1 hour per inch thickness, 2 hour minimum for plate, air cool or quench, depending on section size. Cooling to $800 \mathrm{~F}$ max imum should be within 3 minutes.

This steel can be hardened only by cold working, and th is to a lesser degree than the other steels of the 18-8 family. 


\subsection{JOINING}

Thermal properties of this steel should be considered in the design of welded equipment and welding fixtures to minimize warpage or distortion at the weld. Welding rods of similar chemical analysis, or with slightly higher chromium-nickel content, as the base metal should be used. The use of fluxes, coated rods and inert gas shields is recommended.

\subsection{FORMING}

General. Forming of this steel differs in various respects from that of Types 301 and 302, but it is similar to that of Type 304. Because of its lower strain hardening, Type 305 requires less power and requires fewer intermediate anneals in multi-stage forming. Its stretch forming ability, however, is lower than that of Types 301 and 302, because of its lower elongation.

Deep drawing of this steel is usually performed with the same or less reduction in the first draw than that of Types 301 and 302 , but without intermediate anneals before the second, and possibly further draws. The reductions in these draws should be considerably lower than those possible for Type 301 which needs annealing after each draw. Type 305 also has a greater tendency to become th in at sharp radii than Types 301 and 302 . Annealing is necessary if the hardness exceeds 35 to $40 \mathrm{R}_{\mathrm{c}}$.

Type 305 is preferred for spinning applications because of its lower strain hardening. The surface should be kept clean and free from foreign particles during spinning.

\subsection{MACHINABILITY}

Machining and Grinding. All 300 series austenitic stainless steels can be machined. Use of high speed or carbide tools, positive feed, and abundant flow of coolant or lubricant is recommended. 


\subsection{MECHANICAL PROPERTIES}

\subsection{SHORT TERM}

\subsubsection{Static}

Figure 2.1-1 Effect of Cold Rolling on Tensile Properties

Figure 2.1-2. Effect of Cold Drawing on Tensile Properties of Coiled Bar

Figure 2.1-3 Effect of Cold Rolling on Hardness of Various 18-8 Stainless Steel Sheets

Table 2.1-1 Mechanical Properties in Various Environments

Table 2.1-2 Range of Tensile Properties and Hardness for Various Tempers and Diameters of Cold Draw Wire 

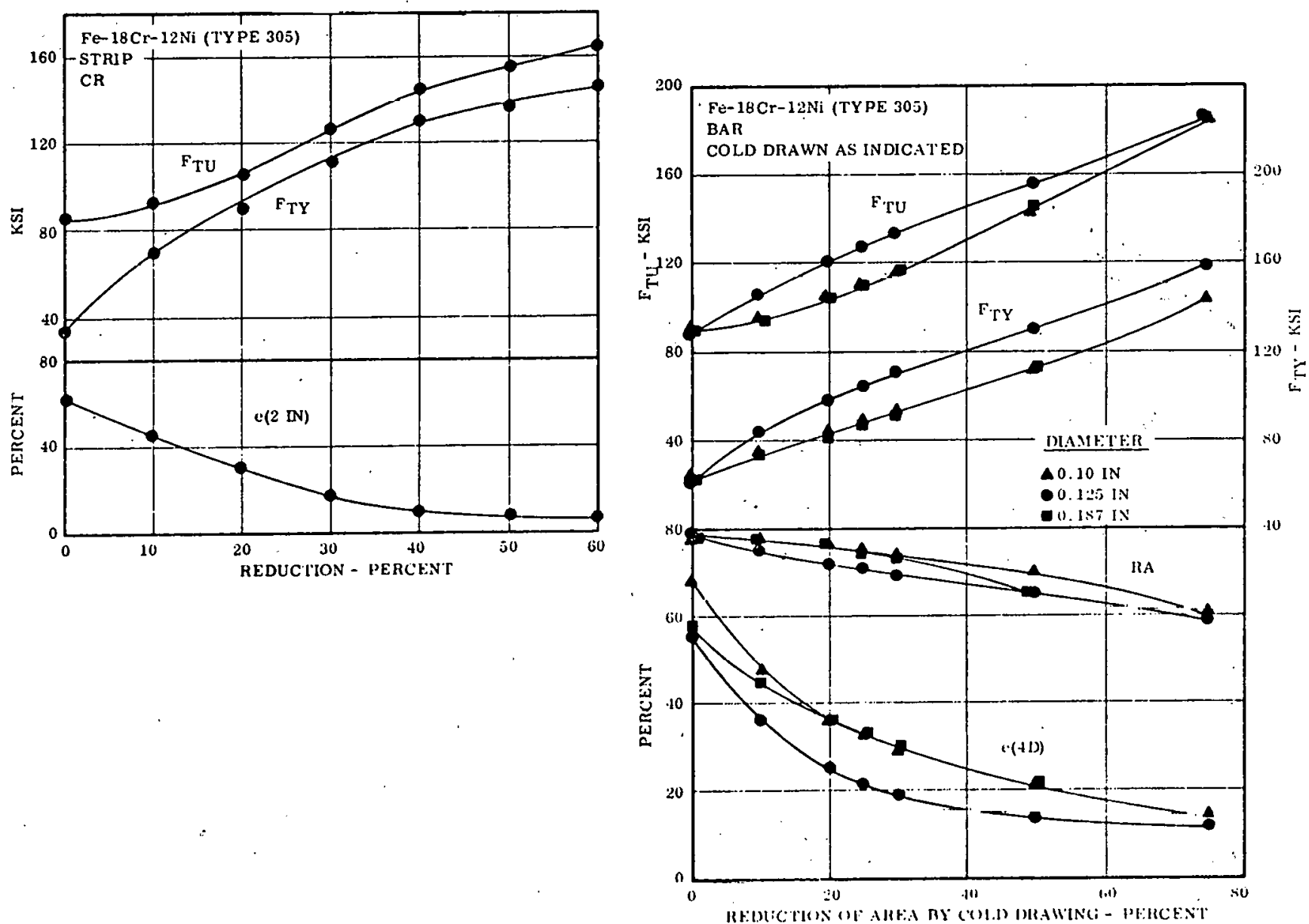

EFFECT OF COLD ROLLING

EFFECT OF COLD DRAWING ON TENSILE PROPERTIES OF STRIP. ON TENSILE PROPERTIES OF BAR.

Figure 2.1-1 


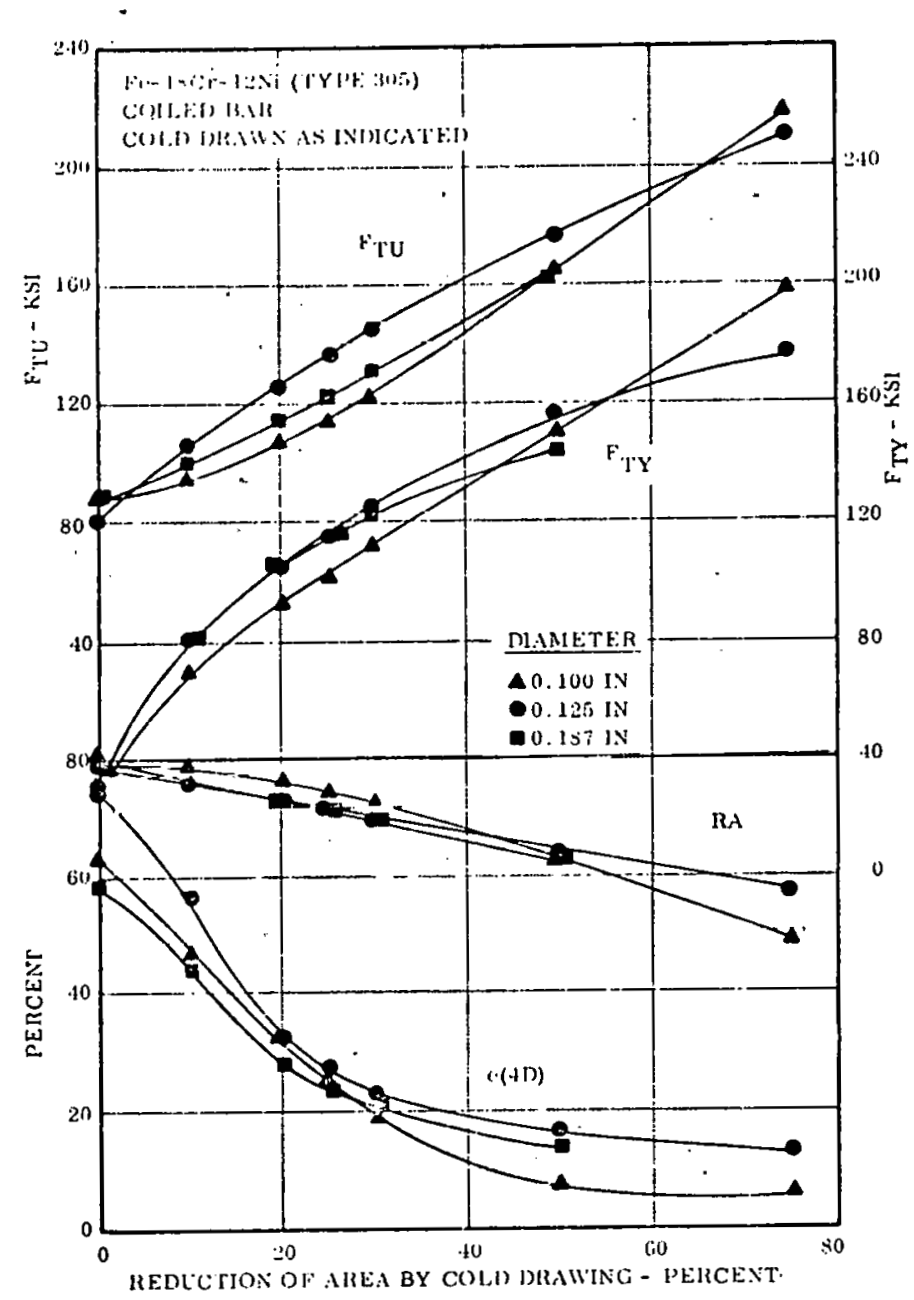

EFFECT OF COLD DRAWING ON TENSILE PROPERTIES OF COILED BAR.

Figure 2.1-2

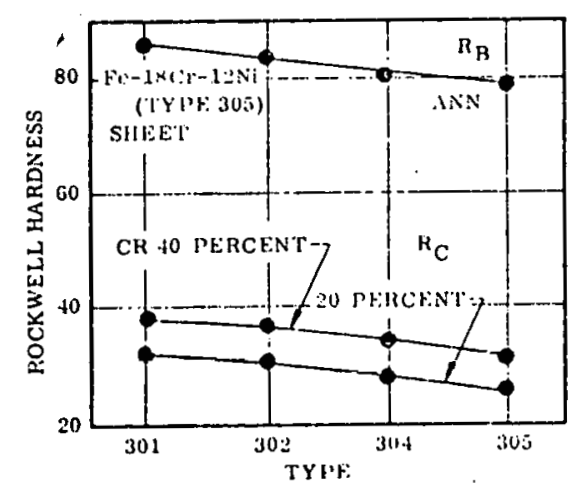

\section{EFFECT OF COLD ROLLING ON} HARDNESS OF.VARIOUS 18-8 STAINLESS STEEL SHEETS.

Figure 2.1-3 
TABLE 2.1-1

MECHANICAL PROPERTIES IN VARIOUS ENVIRONMENTS

\begin{tabular}{|c|c|c|}
\hline Source & \multicolumn{2}{|l|}{ Ref. 6.6} \\
\hline Alloy & \multicolumn{2}{|c|}{$\mathrm{Fe}-18 \mathrm{Cr}-12 \mathrm{Ni}\left(\mathrm{T}_{y p e} 305\right)$} \\
\hline Condition & \multicolumn{2}{|c|}{ Anneal + Cold Drawn } \\
\hline Environment & 10,000 psi He${ }^{*}$ & $10,000 \mathrm{psi} \mathrm{H}_{2}$ \\
\hline$F_{t u}-k s i$ & $90^{*}$ & 87 \\
\hline$F_{\text {ty }}-k s i$ & 51 & - \\
\hline$e(1.25 \mathrm{in})$ - percent & 63 & 65 \\
\hline RA - percent & 78 & 75 \\
\hline $\begin{array}{l}\text { Notch Strength - ksi } \\
\left(K_{f} 8.6\right)\end{array}$ & $165^{*}$ & 147 \\
\hline RA (Notchied Bar) - percent & 19 & 17 \\
\hline $\begin{array}{l}\text { *He was contam lnated with } \\
\text { those for tests in ambient a } \\
\text { for effect of } 10,000 \text { psi pres }\end{array}$ & $\begin{array}{l}\text { Strength rep } \\
\text { inus } 10 \text { ski to }\end{array}$ & nsate \\
\hline
\end{tabular}


TABLE 2.1-2

RANGE OF TENSILE PROPERTIES AND HARDNESS FOR VARIOUS TEMPERS AND DIAMETERS OF COLD DRAW WIRE

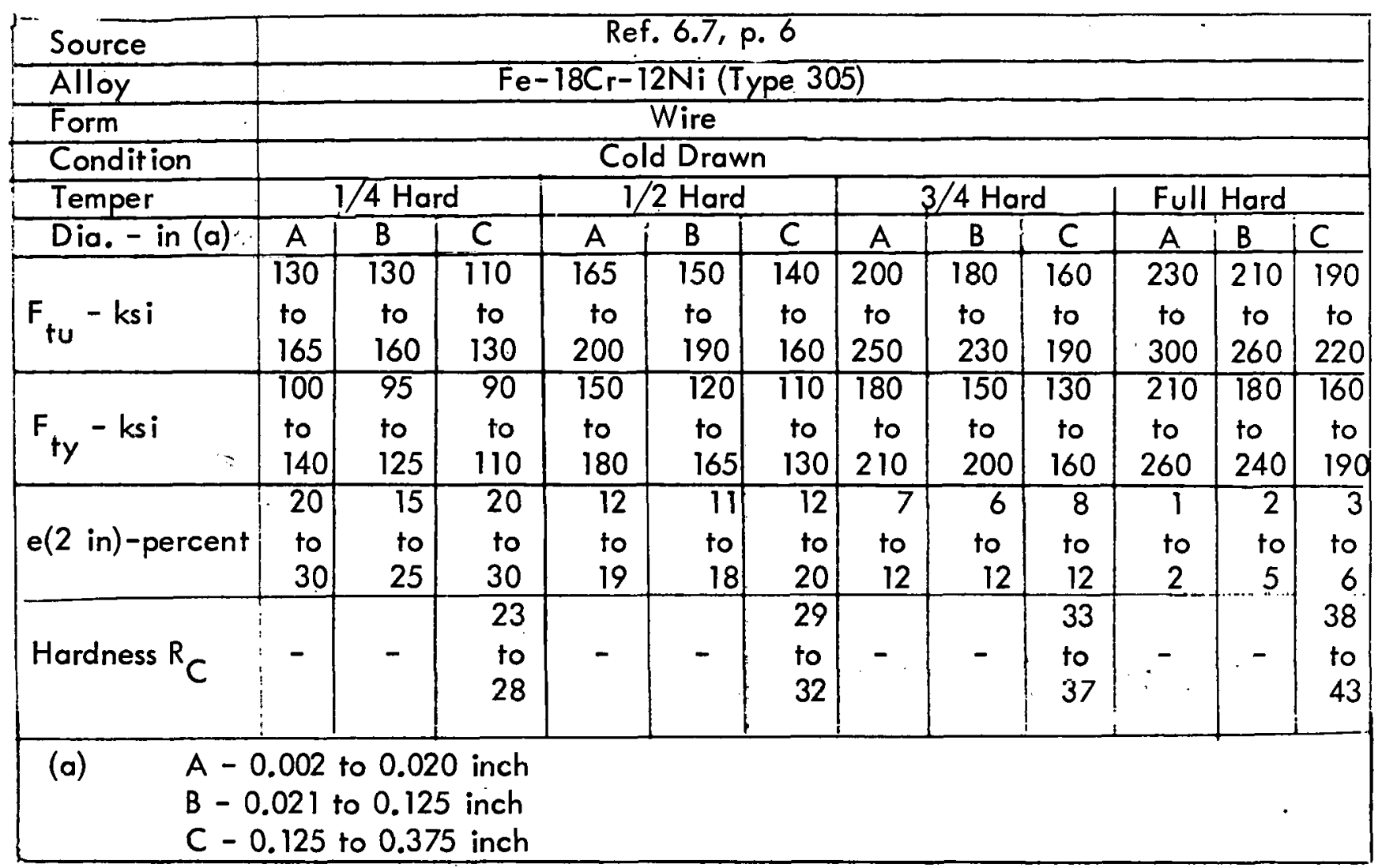




\subsubsection{Dynamic}


2.2 LONG TERM

$3-14$ 


\subsection{CYCLIC LOAD PROPERTIES}

Figure 2.3-1 Fatigue Crack Growth in Annealed Sheet

Figure 2.3-2 Fatigue Crack Growth in Cold Rolled Sheet 


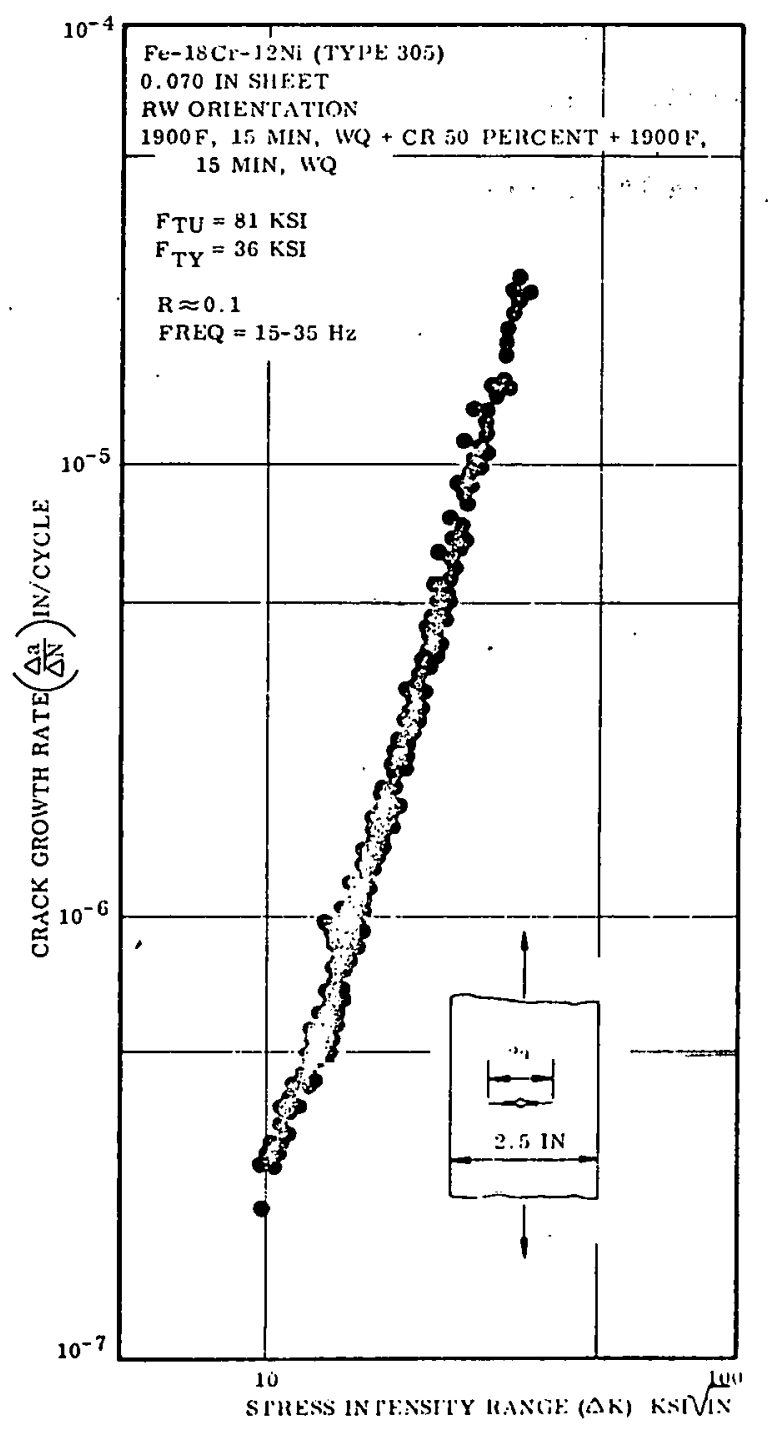

FATIGUE CRACK GROWTH IN ANNEALED SHEET (LONGITUDINAL OR RW ORIENTATION)

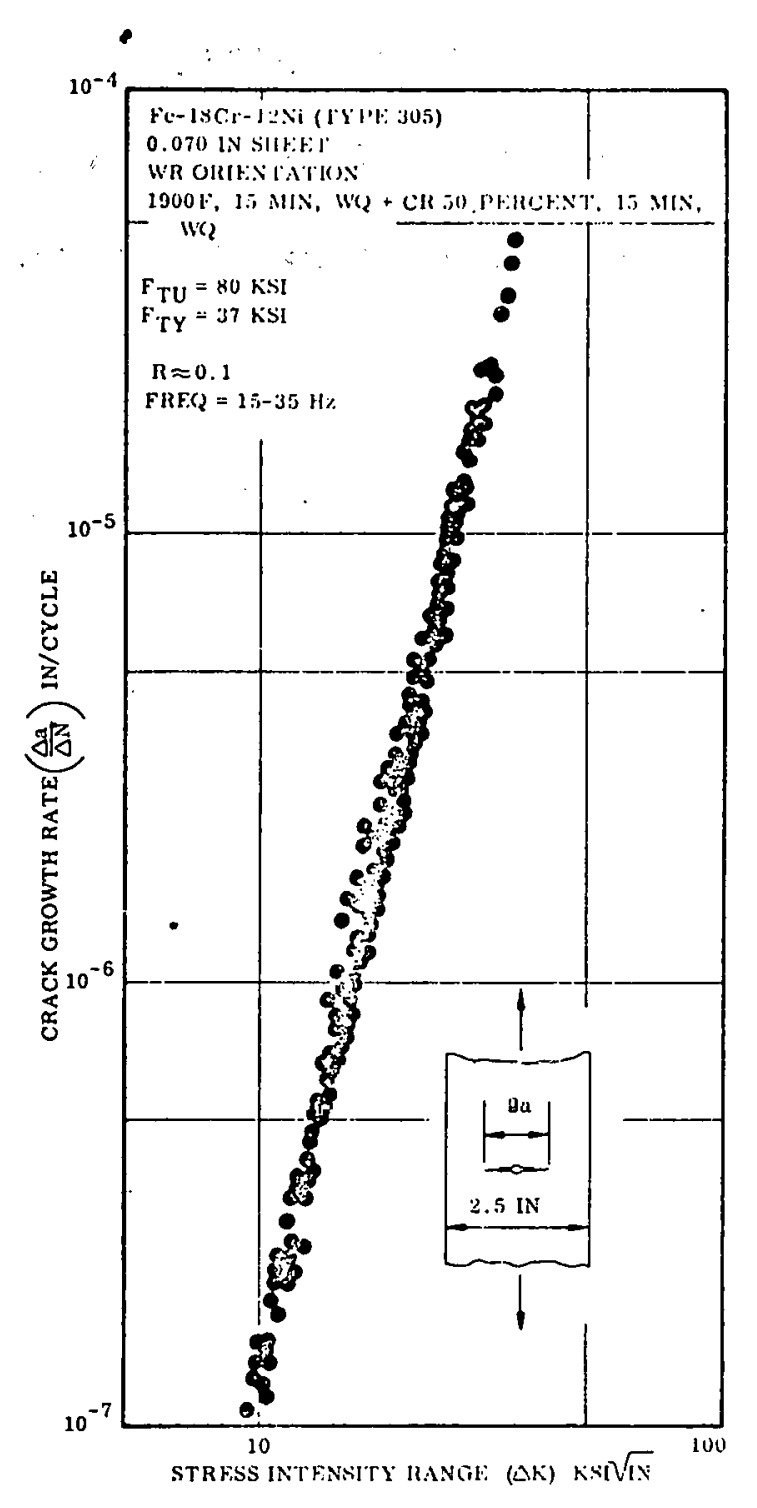

FATIGUE CRACK GROWTH

IN ANNEALED SHEET (TRANSVERSE OF WR ORIENTATION)

Figure 2.3-1 


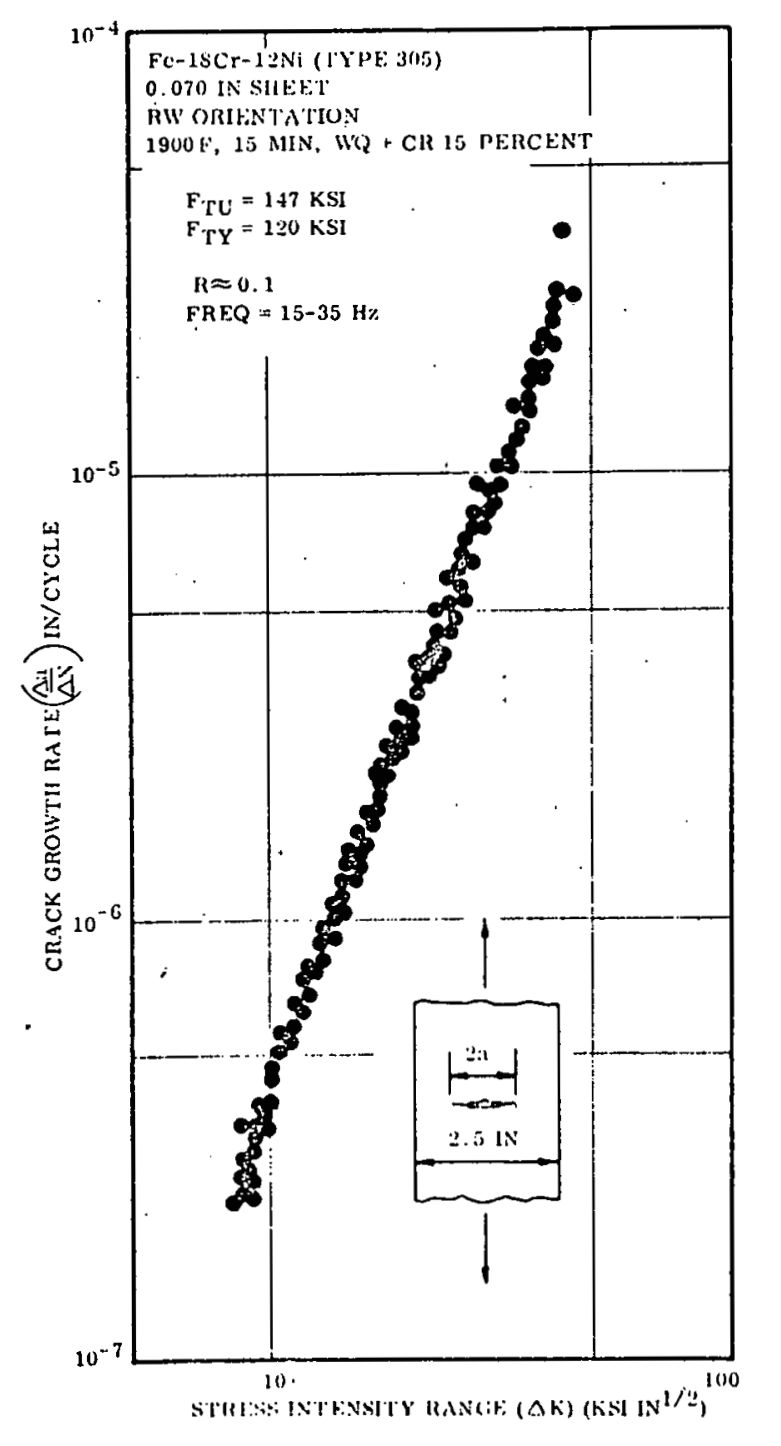

FATIGUE CRACK GROWTH IN COLD ROLLED SHEET (LONGITUDINAL OR RW ORIENTATION)

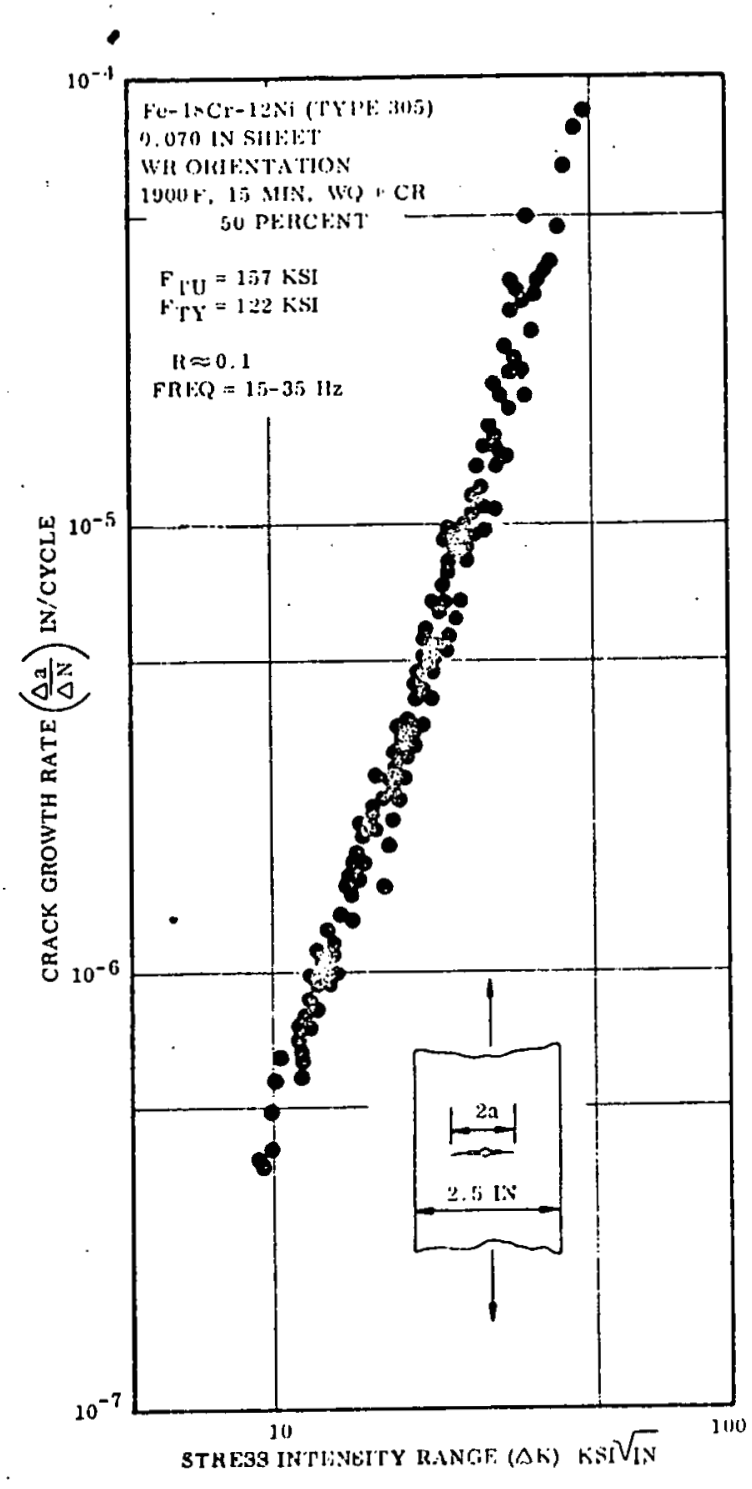

FATIGUE CRACK GROWTH IN COLD ROLLED SHEET (TRANSVERSE OR WR ORIENTATION)

Figure 2.3-2 


\subsection{PHYSICAL PROPERTIES}

3. 1 THERMAL PROPERTIES

Table 3.1-1 Thermal Expansion 
TABLE 3.1-1

THERMAL EXPANSION

\begin{tabular}{|c|c|}
\hline Source & Ref. 6.5 \\
\hline Alloy & $\mathrm{Fe}-18 \mathrm{Cr}_{\mathrm{r}}-12 \mathrm{Ni}$ (Type 305) \\
\hline $\begin{array}{c}\text { Temperature Range } \\
\mathrm{F}\end{array}$ & $\begin{array}{c}\text { Mean Coefficient } \mathrm{f} \text { Ther- } \\
\text { mal Expansion } 10^{-6} \mathrm{in} / \mathrm{in} / \mathrm{F}\end{array}$ \\
\hline $32-212$ & 9.6 \\
$32-600$ & 9.9 \\
$32-1000$ & 10.2 \\
$32-1200$ & 10.4 \\
\hline
\end{tabular}


3.2 ELECTRICAL/MAGNETIC PROPERTIES

Table 3.2-1 Effects of Cold Reduction on Permeability at Various

Temperatures at $\mathrm{H}=200$. Oersteds

Figure 3.2-1 Effect of Cold Reduction on Permeability of Cold Rolled Strip 
TABLE 3.2-1

EFFECT OF COLD REDUCTION ON PERMEABILITY AT

VARIOUS TEMPERATURES AT $\mathrm{H}=200$ OERSTEDS

\begin{tabular}{|c|c|c|}
\hline Source & \multirow{2}{*}{\multicolumn{2}{|c|}{$\frac{\text { Ref, } 6.8}{\mathrm{Fe}-18 \mathrm{Cr}_{\mathrm{r}}-12 \mathrm{Ni} \text { (Type 305)* }}$}} \\
\hline Alloy & & \\
\hline Condition & \multicolumn{2}{|l|}{ Anneal + Cold Reduced } \\
\hline Percent Cold Reduction & Temperature Range $-\mathrm{F}$ & $\begin{array}{l}\text { Permeability at } \\
\mathrm{H}=200 \text { Oersteds }\end{array}$ \\
\hline $\begin{array}{r}0 \\
25\end{array}$ & $\begin{array}{l}20-100 \\
20-300\end{array}$ & $\begin{array}{l}1.003 \\
1.005 \\
1025\end{array}$ \\
\hline $\begin{array}{l}\frac{50}{{ }^{*} \mathrm{C} 1.069, \mathrm{Mn} 0.94, \mathrm{PO} 0} \\
\mathrm{Ni} 11.65\end{array}$ & $\frac{20-8 / 1}{50.013, \mathrm{Si} 0.52, \mathrm{Cr} ! 7.19}$ & 1.023 \\
\hline
\end{tabular}

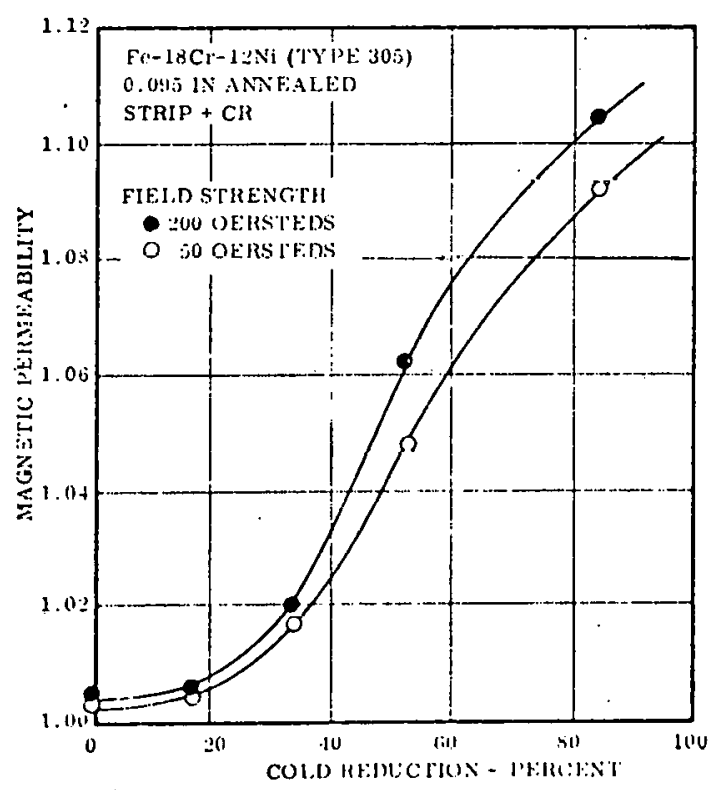

Figure 3.2-1 EFFECT OF COLD REDUCTION ON PERMEABILITY OF COLD ROLLED STRIP. 
4.0 CHEMICAL PROPERTIES 


\subsection{NUCLEAR PROPERTIES}

Effect of neutron irradiation on room temperature tensile properties of annealed bar, see Section 1.4.5. 


\subsection{REFERENCES}

6.1 Aerospace Structural Metals Handbook, Vol. 1, AFML-TR-68-115, Mechanical Properties Data Center, Air Force Materials Information Center, Belform Stulen,Inc., Traverse City, Michigan, 49684 (1972 - latest revision, 1975).

6.2 J. E. Hauck, ed., 1974 Materials Selector, Materials Engineering, Vol. 78, No. 4, Mid-September 1973, p. 46, (Reinhold Publishing Co. - Stanford, Connecticut).

6.3 H. E. Chandler, ed., Metal Progress - Databook 1975, Vol. 108, No. 1, Mid-June 1975, p. 205, (American Society for Metals - Metal Park, Ohio).

6.4 Wilson, J. C. and Berggren, R. G.,"Effects of Neutron Irradiation in Șteel," Proc. ASTM, 55 (1955), p. 689.

6.5 Republic Steel Corporation, "Republic ENDURO Stainless Steel - ChromiumNickel Types, 300 Series," Adv. 1124R4-15M-3-66 (1966).

6.6 Walter, R. J., Jewett, R. P., and Chandler, W. T., "On the Mechanism of Hydrogen-Environment Embrittlement of Iron and Nickel-base Alloys," Materials Science and Engineering, 3 (1969//0), pp. $98-110$.

6.7 Chromium-Nickel Stainless Steel Data, Section 1, Bulletin A, International Nickel Co., N. Y. (1963).

6.8 Kess, L., Allegheny Ludlum Industries, "Personal Communication to W. F. Brown, Jr.," September 18, 1970.

6.9 AMS 5514A, Plate, Sheet and Strip $-18 \mathrm{Cr}-11 \mathrm{Ni}$, Deep Drawing and Spinning (February 15, 1952).

6.10 AMS 5685D, Wire, Safety $-18 \mathrm{Cr}-11.5 \mathrm{Ni}$, Solution Heat Treated (May 1, 1968).

6.11 AMS 5686A, Wire, Riveting - 18CR-11Ni, Solution Heat Treated (February 15, 1952).

6.12 ASTM A167-63, Standard Specification for Corrosion-Resisting ChromiumNickel Steel Plate, Sheet, and Strip (September 30, 1963).

ASTM A240-67, Standard Specification for Chromium and ChromiumNickel Stainless Steel Plate, Sheet, and Strip for Fusion-Welded Unfired Pressure Vessels (December 12, 1967). 


\section{REFERENCES (Continued)}

6.14 ASTM A276-67, Standard Specification for Stainless and Heat-Resisting Steel Bars and Shapes (December 12, 1967).

6.15 ASTM A314-63, Standard Specification for Stainless and Heat-Resisting Steel Billets and Bars for Forging (September 30, 1963).

6.16 ASTM A473-63, Standard Specification for Stainless and Heat-Resisting Steel Forgings (September 30, 1963).

6.17 ASTM A249-65, Standard Specification for Welded Austenitic Stainless Steel Boiler, Superheater, Heat-Exchanger, and Condenser Tubes (August 31, 1965).

6.18 ASTM A478-67, Standard Specification for Chromium-Nickel Stainless and Heat-Resisting Steel Weaving Wires (March 6, 1967).

6.19 ASTM A492-67, Standard Specification for Stainless and Heat-Resisting Steel Rope Wire (March 6, 1967).

6.20 ASTM A493-68, Standard Specification for Stainless and Heat-Resisting Steel for Cold Heading and Cold Forging - Bar and Wire (February 14, 1968).

6.21 ASTM A580-67, Standard Specification for Stainless and Heat-Resisting Steel Wire (December 12, 1967). 
SECTION 4.0

316 STAINLESS STEEL 


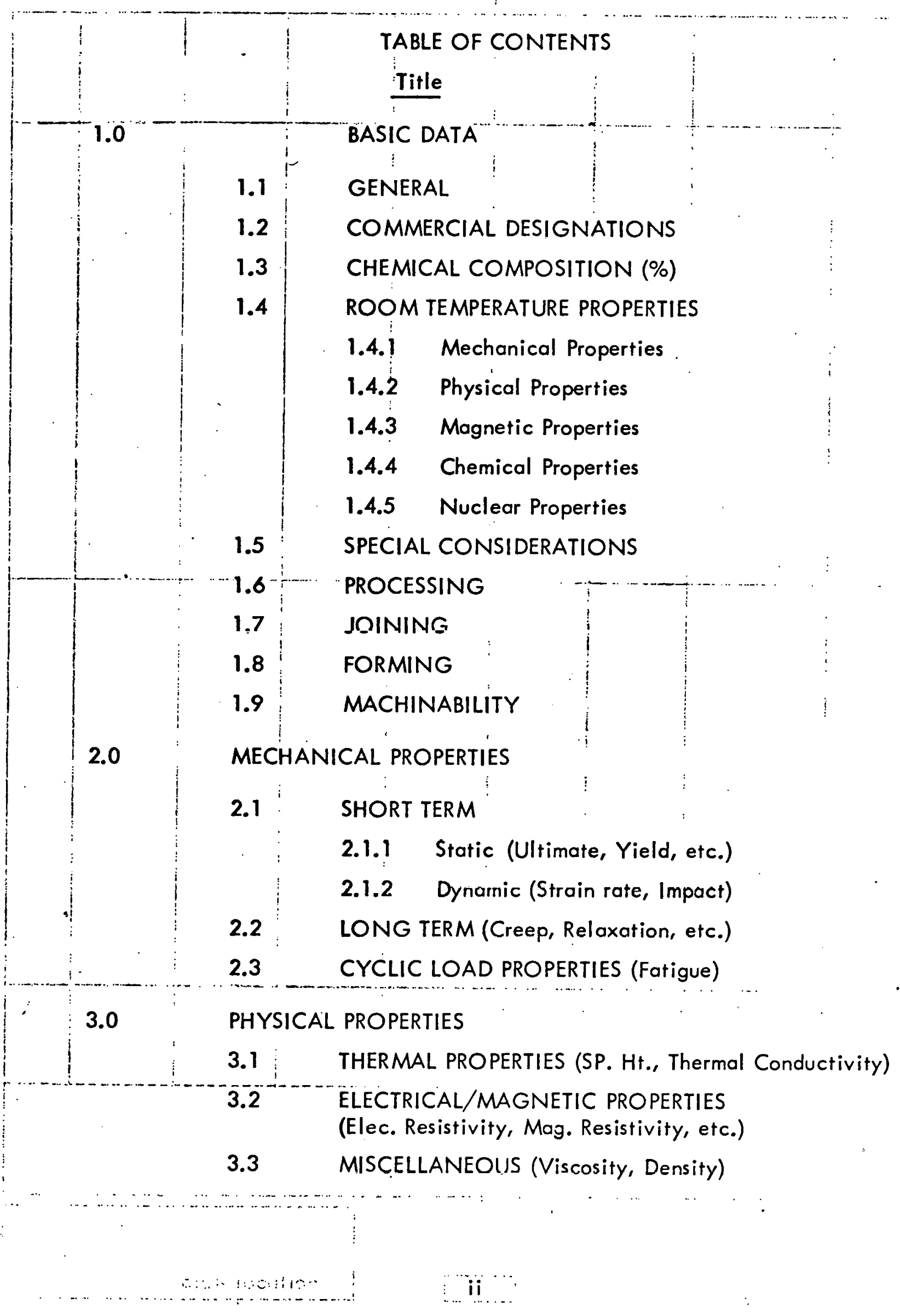




$\begin{array}{cc}4.0 & \text { CHEMICAL PROPERTIES } \\ 5.0 & \text { NUCLEAR PROPERTIES } \\ 6.0 & \vdots \\ & \text { REFERENCES }\end{array}$




\section{I.0 BASIC DATA}

\subsection{GENERAL}

AISI 316 is an austenitic stainless steel of the 18-8 class, the chemistry having been modified by the addition of molybdenum. As a result, th is alloy has a corrosion resistance superior to that of other austenitic steel grades for certain applications.

The property values given in Section 1.4 are averages, exept for ultimate tensile strength, tensile yield strength, elongation, and reduction of area, which are $80 \%$ of average.

\subsection{COMMERCIAL DESIGNATIONS}

Wrought: $18-8$ Mo Stainless Steel

Cast: CF3M, CF8M, CFI2M (A. C. I. Designations)

Available Forms, Specifications, Suppliers

\begin{tabular}{|c|c|}
\hline Forms and Conditions & Specifications \\
\hline Sheet, Strip and Plate & $\begin{array}{l}M I L-S-5059 \\
Q Q-S-766 \\
\text { AMS 5524 }\end{array}$ \\
\hline Bar, Forgings, Forging Stock & $\begin{array}{l}\text { MIL-S-7720 } \\
\text { QQ-S-763 } \\
\text { AMS 5648 } \\
\end{array}$ \\
\hline Spring Wire & $Q Q-W-423$ \\
\hline Tubing, Seamless & AMS 5573 \\
\hline
\end{tabular}

Suppliers

Allegheny Ludlum Steel Co.

Crucible Steel Co,

Republic Steel Co.

U. S. Steel Co. 


\subsection{CHEMICAL COMPOSITION (\%)}

$\begin{array}{lcccccccc}\text { ELEMENT } & \mathrm{C} & \mathrm{Mn} & \mathrm{Si} & \mathrm{P} & \mathrm{S} & \mathrm{Cr} & \mathrm{Ni} & \mathrm{Mo} \\ \text { MAXIMUM } & 0.08 & 2.00 & 1.00 & .045 & .030 & 18.0 & 14.0 & 3.00 \\ \text { MINIMUM } & - & - & - & - & - & 16.0 & 10.0 & 2.00\end{array}$

I.4 ROOM TEMPERATURE PROPERTIES

(Average Unless Otherwise Noted)

1.4.1 Mechanical Properties

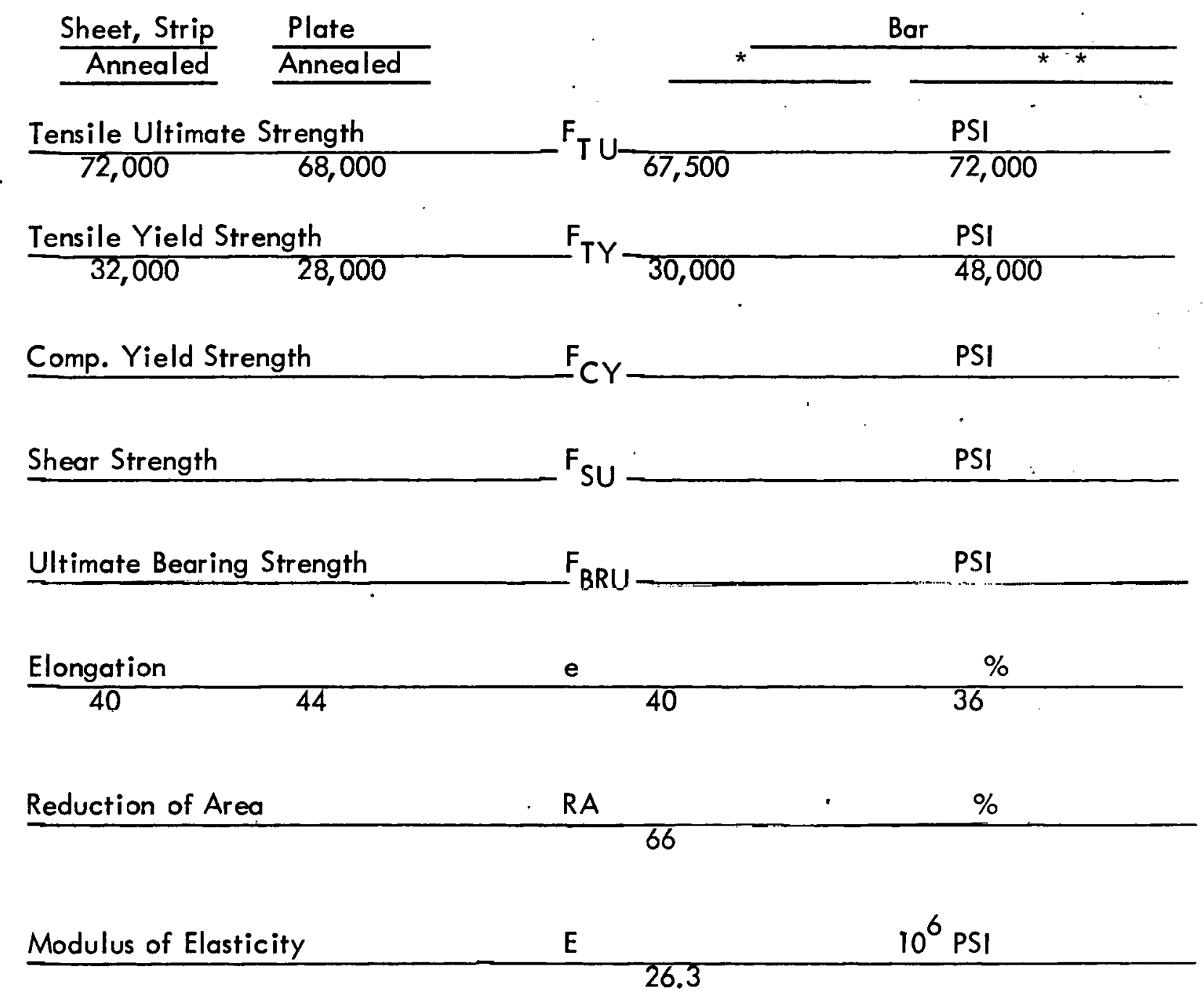




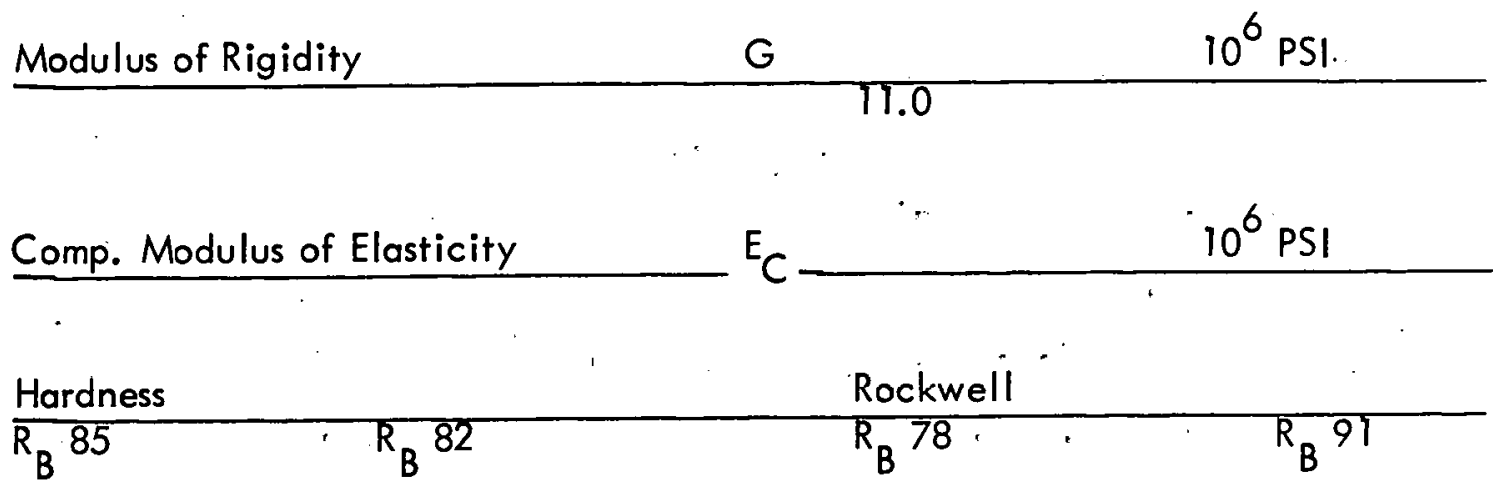

Plastic Bending Modulus

${ }^{*}$ Cold Worked $13 \%$ Before Annealing

**Cold Drawn, 1-in. Dia.

\subsubsection{Physical Properties}

$\begin{array}{lll}\text { Density } & \rho & \mathrm{LB} / \mathrm{IN} \mathrm{N}^{3} \\ & 0.288\end{array}$

Specific Heat

C

BTU/LB $-{ }^{\circ} \mathrm{F}$

10.5

\begin{tabular}{lll} 
Thermal Conductivity & $k$ & $\frac{\mathrm{BTU}-\mathrm{FT}}{\mathrm{HR}-\mathrm{FT}^{2}-{ }^{\circ} \mathrm{F}}$ \\
\hline & 7.87 & $\frac{10^{6} \mathrm{IN} .}{1 \mathrm{~N} .{ }^{\circ} \mathrm{F}}$ \\
\hline Mean Coeff. Lin. Thermal Expans. & $\alpha$ & \\
\hline Electrical Resistivity & 8.3 & \\
\hline & $\mathrm{R}$ & MICROHM $-\mathrm{IN}$. \\
\hline
\end{tabular}

\subsubsection{Magnetic Properties}

Non-magnetic in annealed condition. Formation of ferrite in severely cold worked material will result in very small increase in permeability. 


\subsubsection{Chemical Properties}

Superior corrosion resistance, when compared to other austenitic stainless steels, on exposure to sea water and conditions where particles are deposited on surface.

Oxidation resistance good for continuous service up to $1600^{\circ} \mathrm{F}$ and for intermittent service up to $1500^{\circ} \mathrm{F}$. Scales severely at approximately $1650^{\circ} \mathrm{F}$. This temperature varies with type of atmosphere and other conditions.

\subsubsection{Nuclear Properties}

Austenitic stainless steels retain their high impact strength after irradiation.

\subsection{SPECIAL CONSIDERATIONS}

\section{Service Limits}

$-423^{\circ} \mathrm{F}$ to $1600^{\circ} \mathrm{F}$ (Continuous)

$-423^{\circ} \mathrm{F}$ to $1500^{\circ} \mathrm{F}$ (Intermittent)

\subsection{PROCESSING}

\section{Surface Treatment}

Scale may be removed by a solution of 15 to 20 percent nitric acid and 1 to 3 percent hydrofluoric acid at 120 to $140 \mathrm{~F}$, for 20 to 30 minutes. Scale removal is more easily accomplished when parts have been heated in air.

Chlorinated hydrocarbon cleaners, such as trichloroethylene, should be avoided since they may contribute to stress-corrosion cracking.

\section{Thermal Treatment}

Avoid prolonged heating at temperatures from $800^{\circ}$ to $1600^{\circ} \mathrm{F}$. All heating should be conducted in air or inert atmospheres such as helium, argon, or dissociated ammonia. 


\section{Annealing}

Wrought Products:

$1850-2150^{\circ} \mathrm{F}$, air cool or quench depending on section size. $1950^{\circ} \mathrm{F}$ minimum for sheet.

Casting:

$1950-2100^{\circ} \mathrm{F}$, air cool or quench. Use low side of temperature range for $\mathrm{CF}-8 \mathrm{M}$;

High side (above $2000^{\circ} \mathrm{F}$ ) for CF-12M.

Stabilizing for High-Temperature Service

$1625-1675^{\circ} \mathrm{F}, 2-4 \mathrm{hr}$. furnace or air cool

Stress Relief

$400-750^{\circ} \mathrm{F}, \mathrm{l} / 2$ to $2 \mathrm{hr}$

Alloy can only be hardened by cold work.

\subsection{JOINING}

May be welded by conventional techniques. Oxyacetylene welding is not advisable for castings because of possible impairment of corrosion resistance due to carbon pick-up and possibility of sensitization.

Welding

For parent metal welded to itself, select one of the following weld rods:

AISI 316/MIL-R 503I, CL. 4

AISI 316 ELC/MIL-R-5031, CL. 17

ER 316/ASTM-A-371, CL. ER316

ER 316L/ASTM-A-37I, CL. ER316L

\subsection{FORMING}

Forming should be done at room or forging temperatures, but not in the carbide precipitation range. 
1.9 MACHINABILITY

About $50 \%$ of that of mild carbon steels. 


\subsection{MECHANICAL PROPERTIES \\ (As Function of Temperature)}

\section{I SHORT TERM}

\subsubsection{Static}

Figure 2. 1-1

Figure 2. 1-2

Figure 2.1-3

Figure 2. 1-4

Figure 2.1-5

Figure 2.1-6

Figure 2.1-7

Figure 2.1-8
Ultimate Tensile Strength (Minimum Expected)

Yield Strength (Minimum Expected)

Total Elongation (Minimum Expected)

Impact Strength Rockwell Hardness

Uniform Elongation (Minimum Expected)

Poisson's Ratio

Young's Modulus

Shear Modulus 


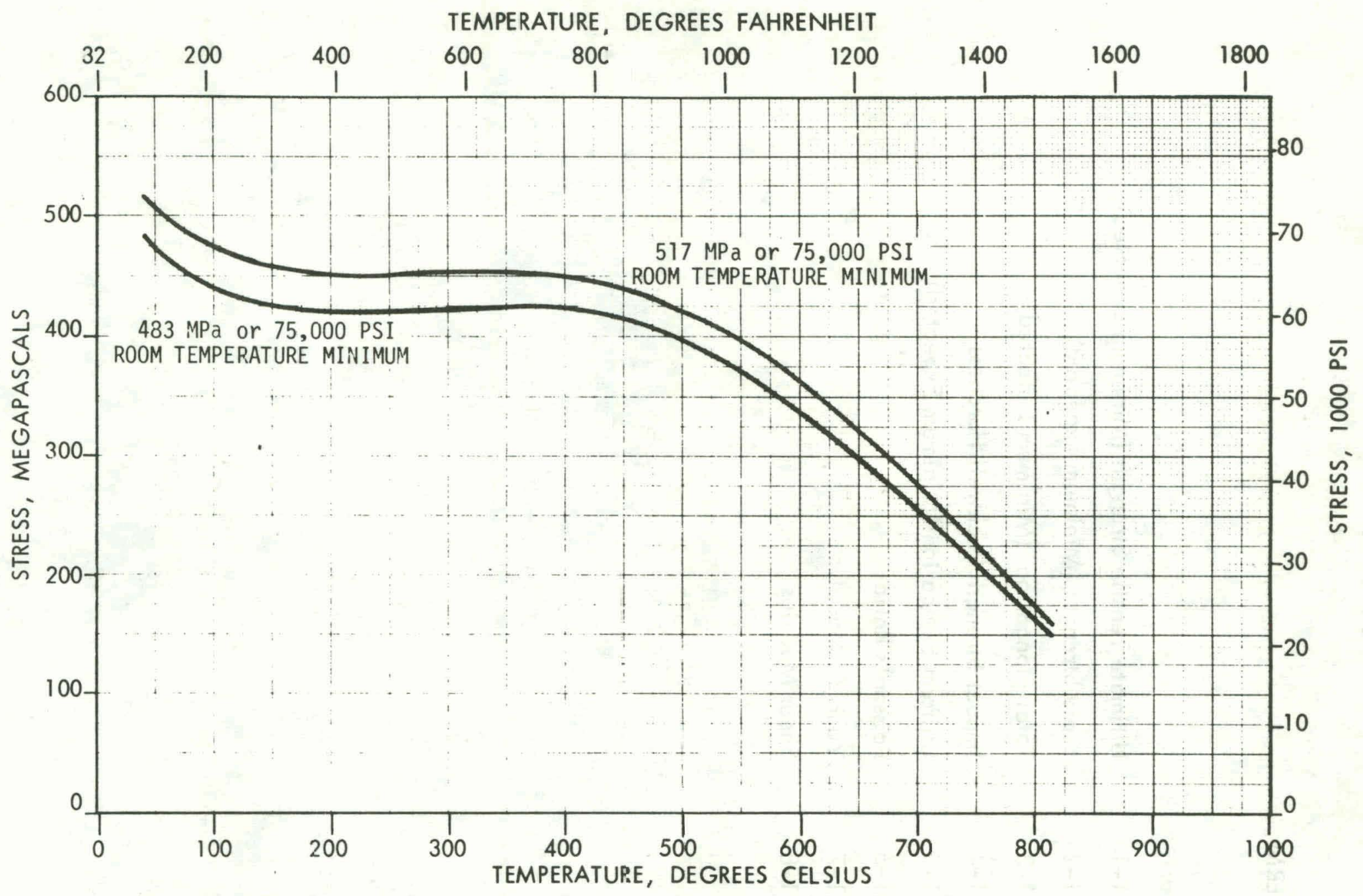




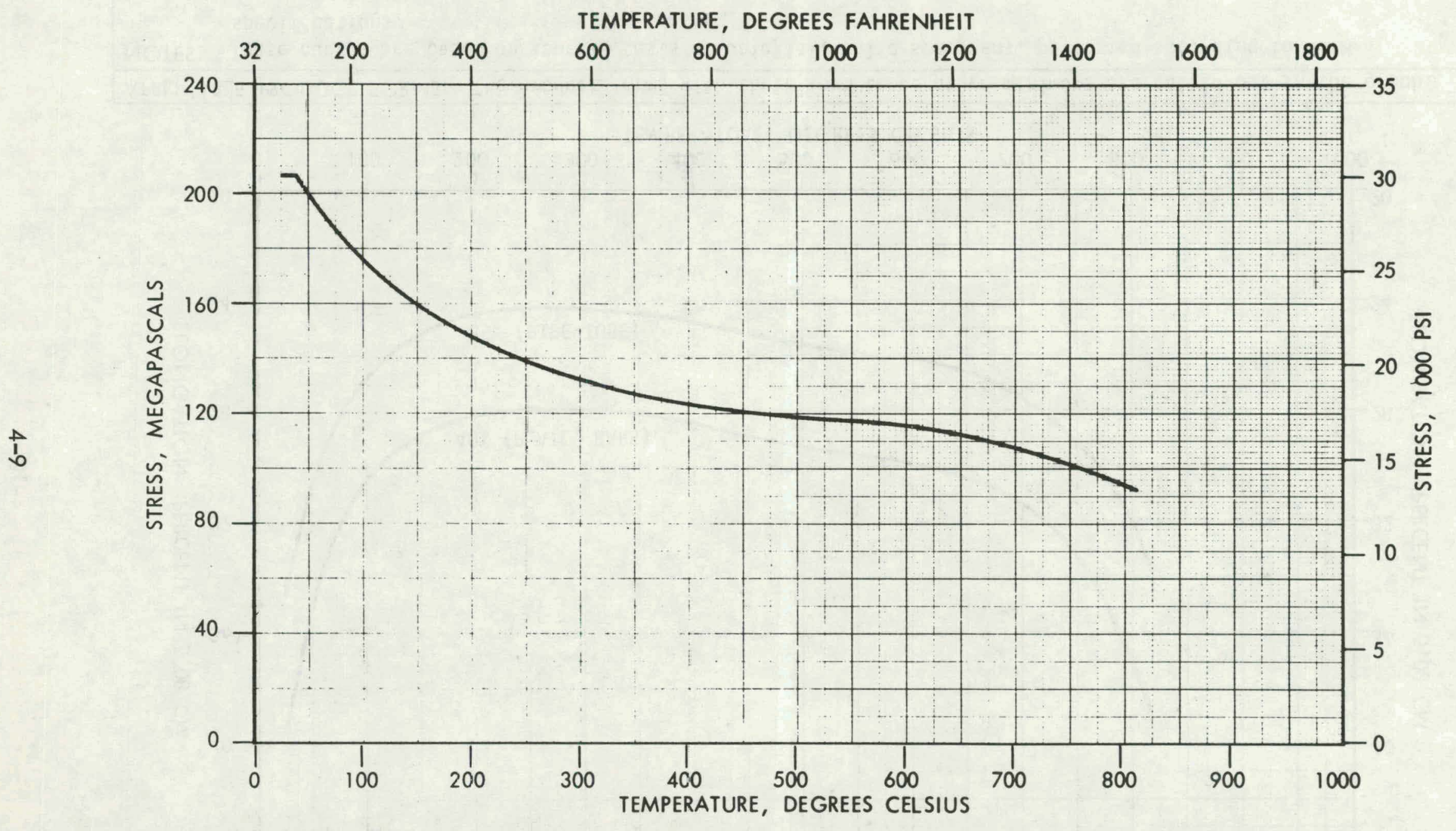

APPLICABLE PRODUCT FORMS: All product forms (except fuel clad).

NOTES: Use of these values above 538 Deg C (10cio Deg F) is limited to material with carbon content greater than or equal to 0.04 percent. This curve was normalized to a room temperature minimum of $207 \mathrm{MPa}(30,000 \mathrm{PSI})$.

Figure 2.1-2 Yield Strength (Minimum Expected) 


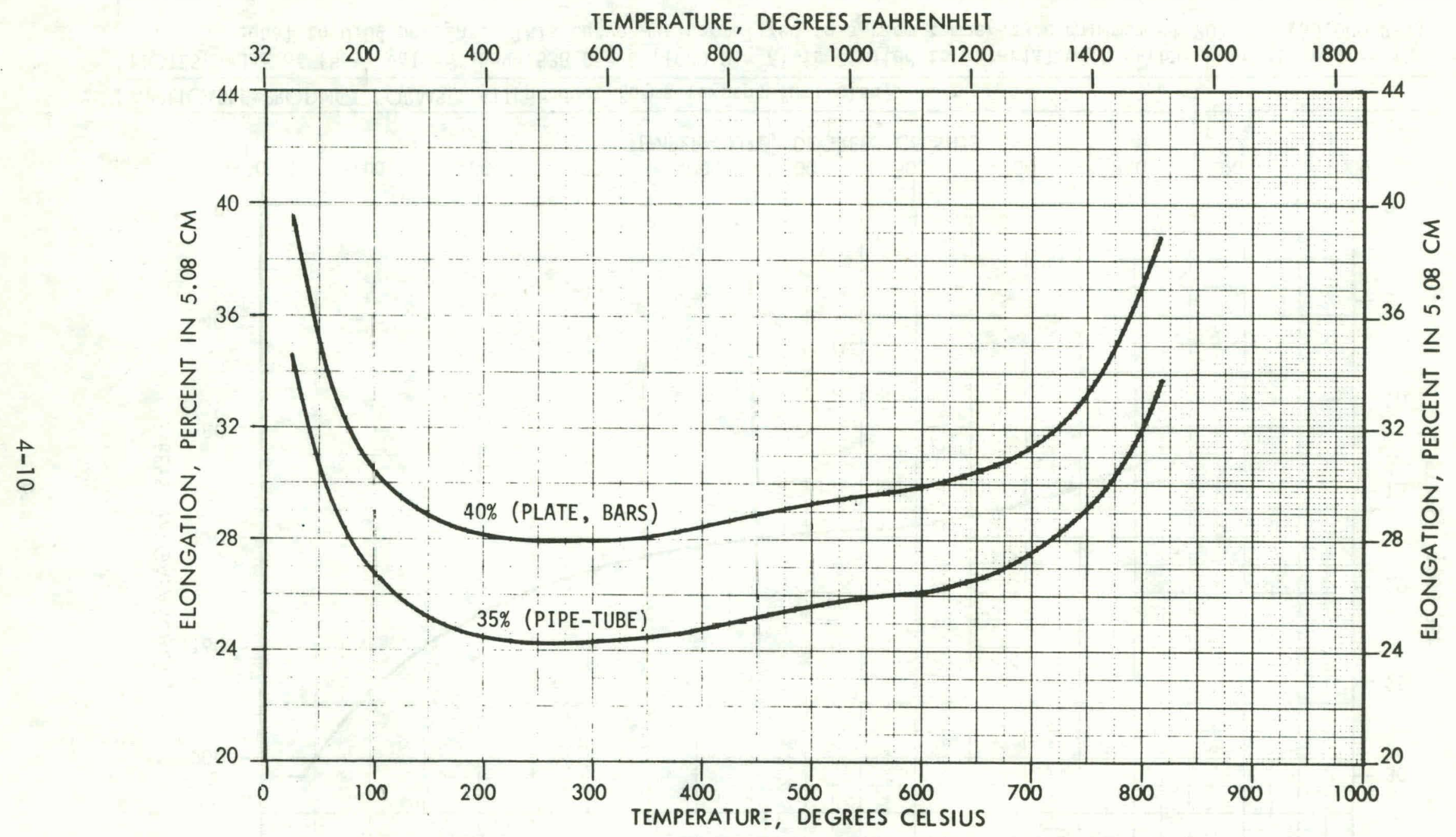

APPLICABLE PRODUCT FORMS: The product forms with their room temperature minimums are called out in the graph. NOTES: These curves are based on tensile tests of uniaxial, solid specimens, performed according to ASTM specificatiors. 

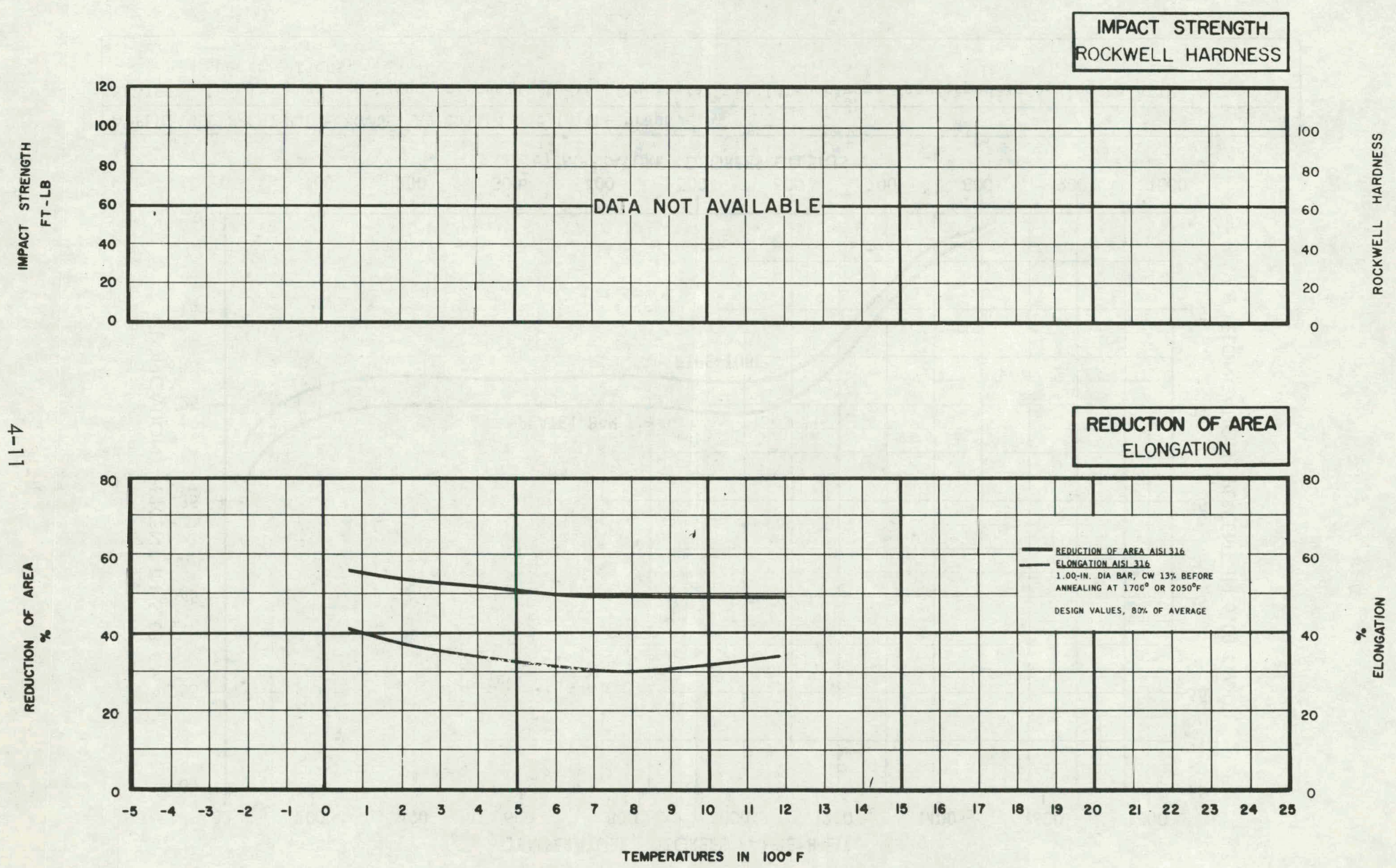

Figure 2.1-4 Impass Strength Rockwell Hardness 


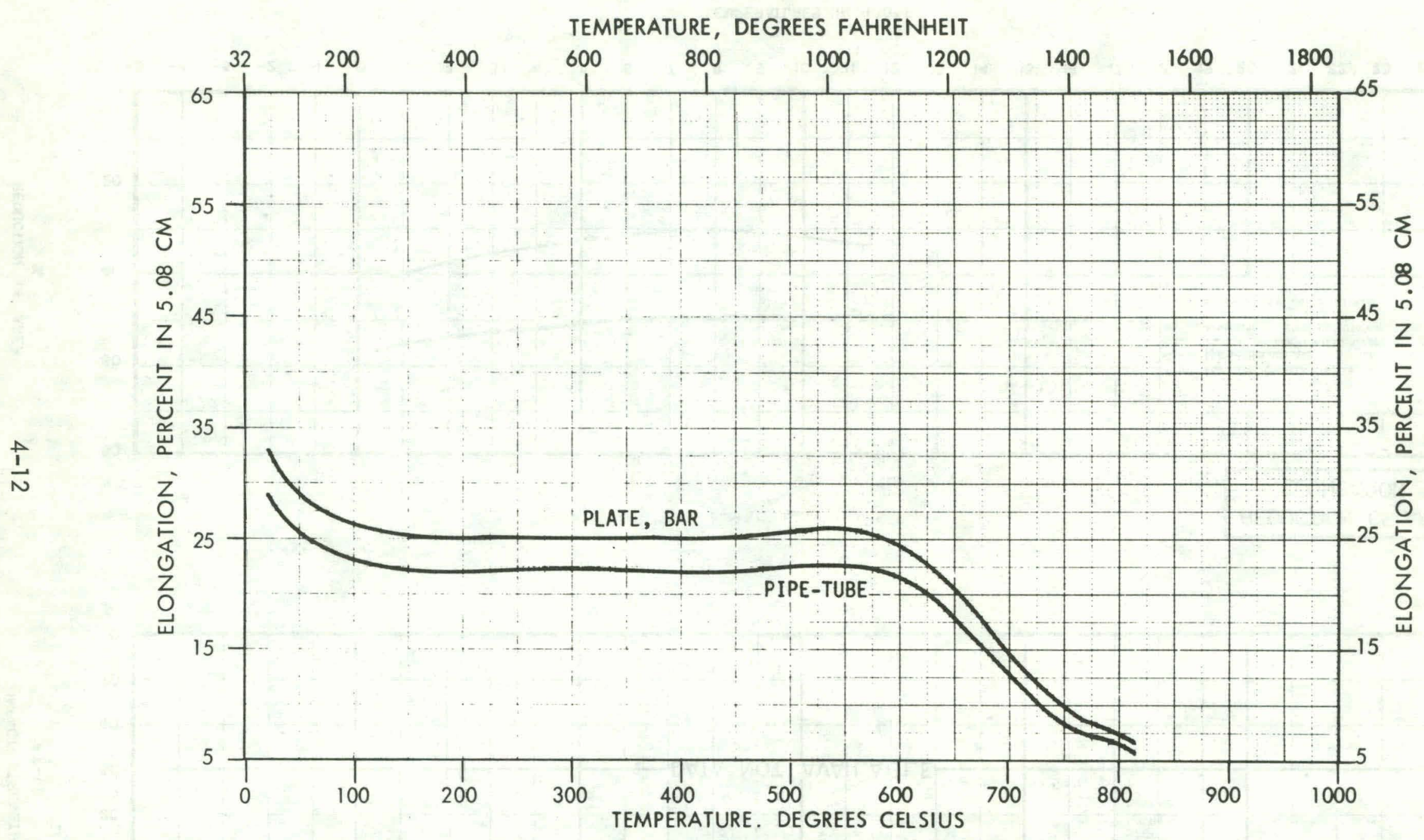

APPLICABLE PRODUCT FORMS: As called out in the graph.

NOTES: These curves are based on tensile tests of uniaxial, solid specimens, performed according to ASTM specifications.

Figure 2.1-5 Uniform Elongation (Minimum Expected) 


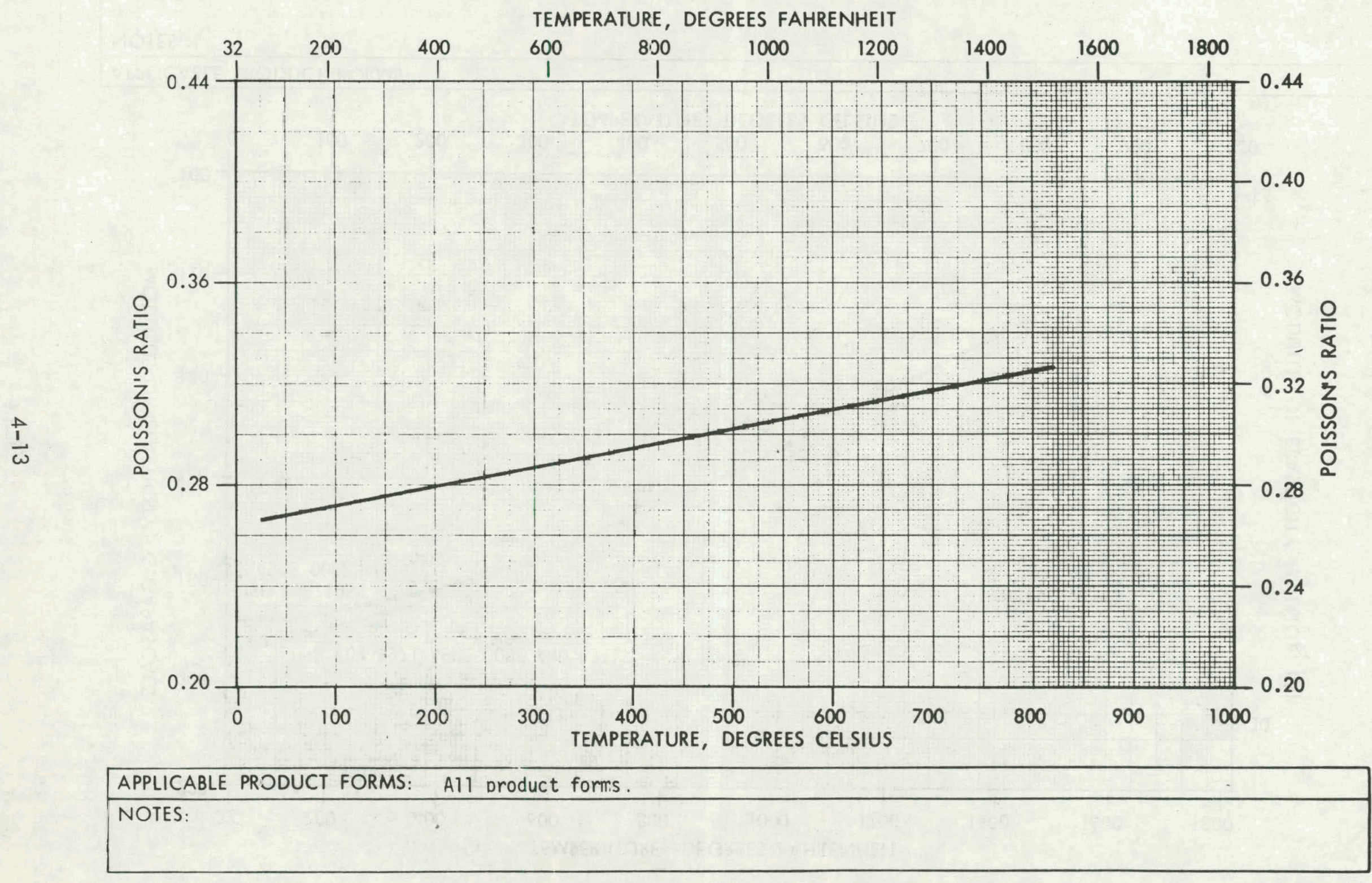

Figure 2.1-6 Poisson's Ratio 
TEMPERATURE, DEGREES FAHRENHEIT

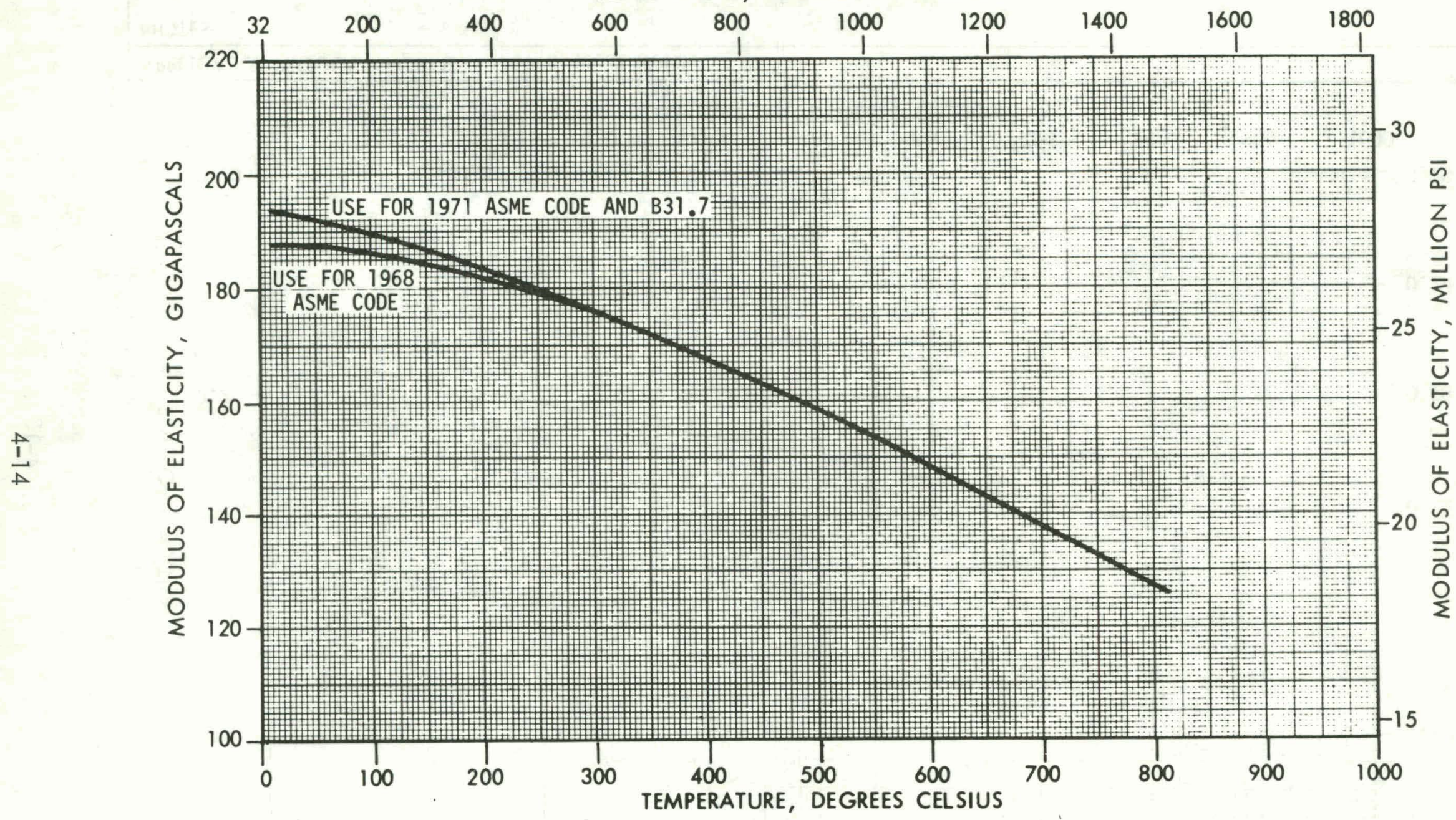

APPLICABLE PRODUCT FORMS:

NOTES:

Figure 2.1-7 Young's Modulus 


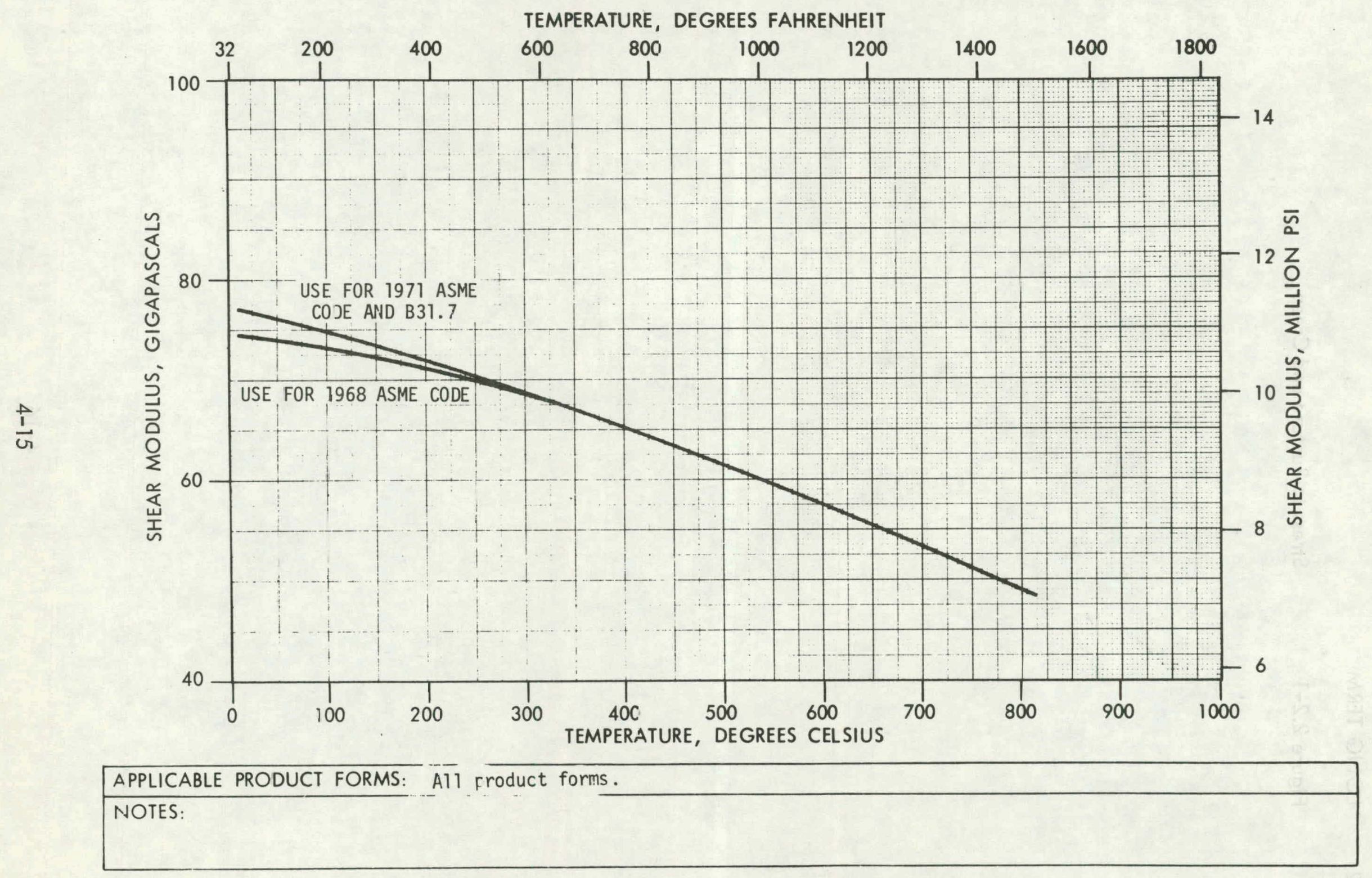

Figure 2.1-8 Shear Modulus 
2.2 LONG TERM

Figure 2.2

4-16 


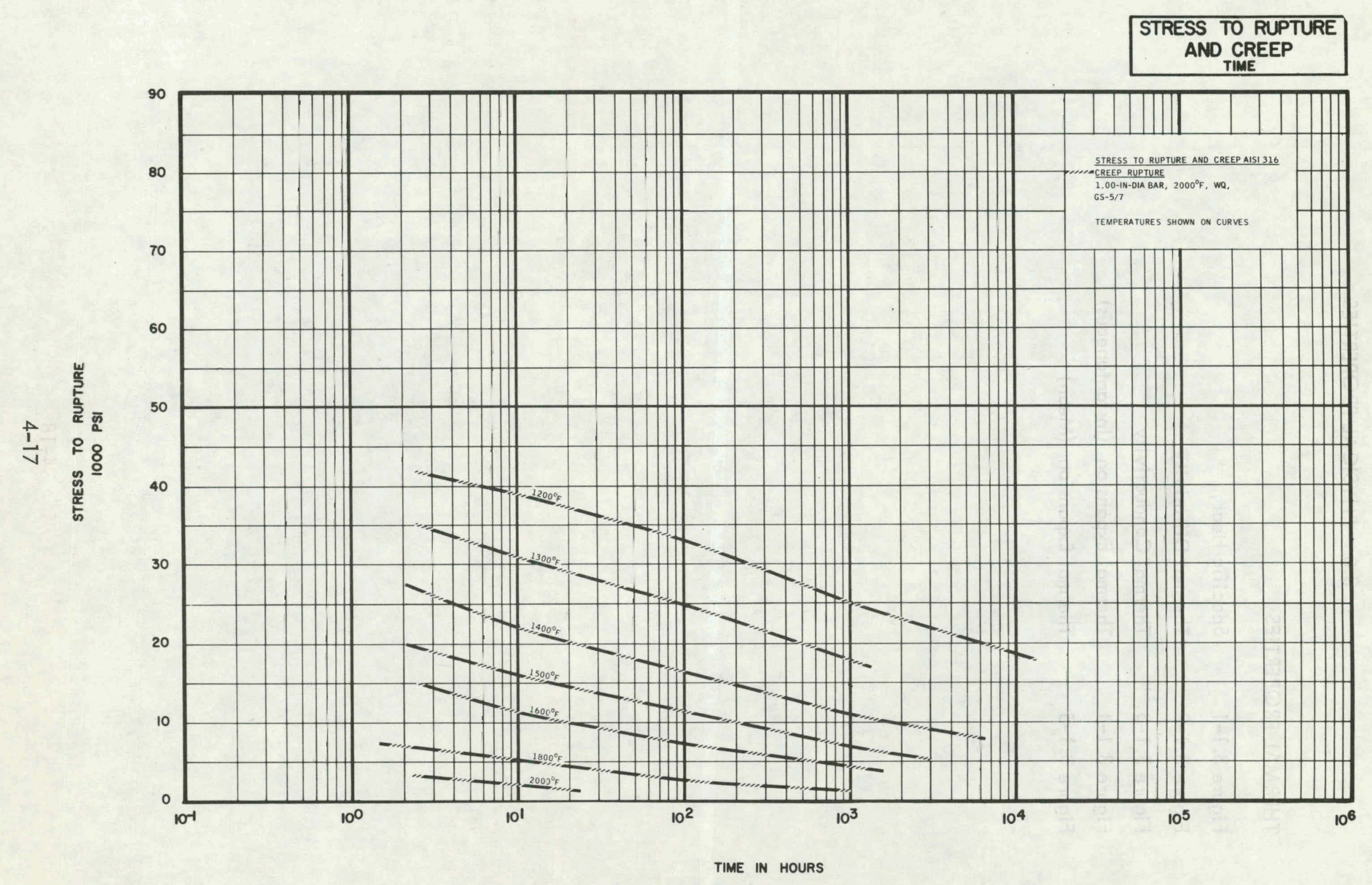

Figure 2.2-1 Stress to Rupture and Creep 


\subsection{PHYSICAL PROPERTIES}

3.1 THERMAL PROPERTIES

Figure 3.1-1 Specific Heat

Figure 3.1-2 Thermal Diffusivity

Figure 3.1-3 Thermal Conductivity

Figure 3.1-4 Thermal Expansion (Instantaneous)

Figure 3.1-5 Thermal Expansion (Mean) 


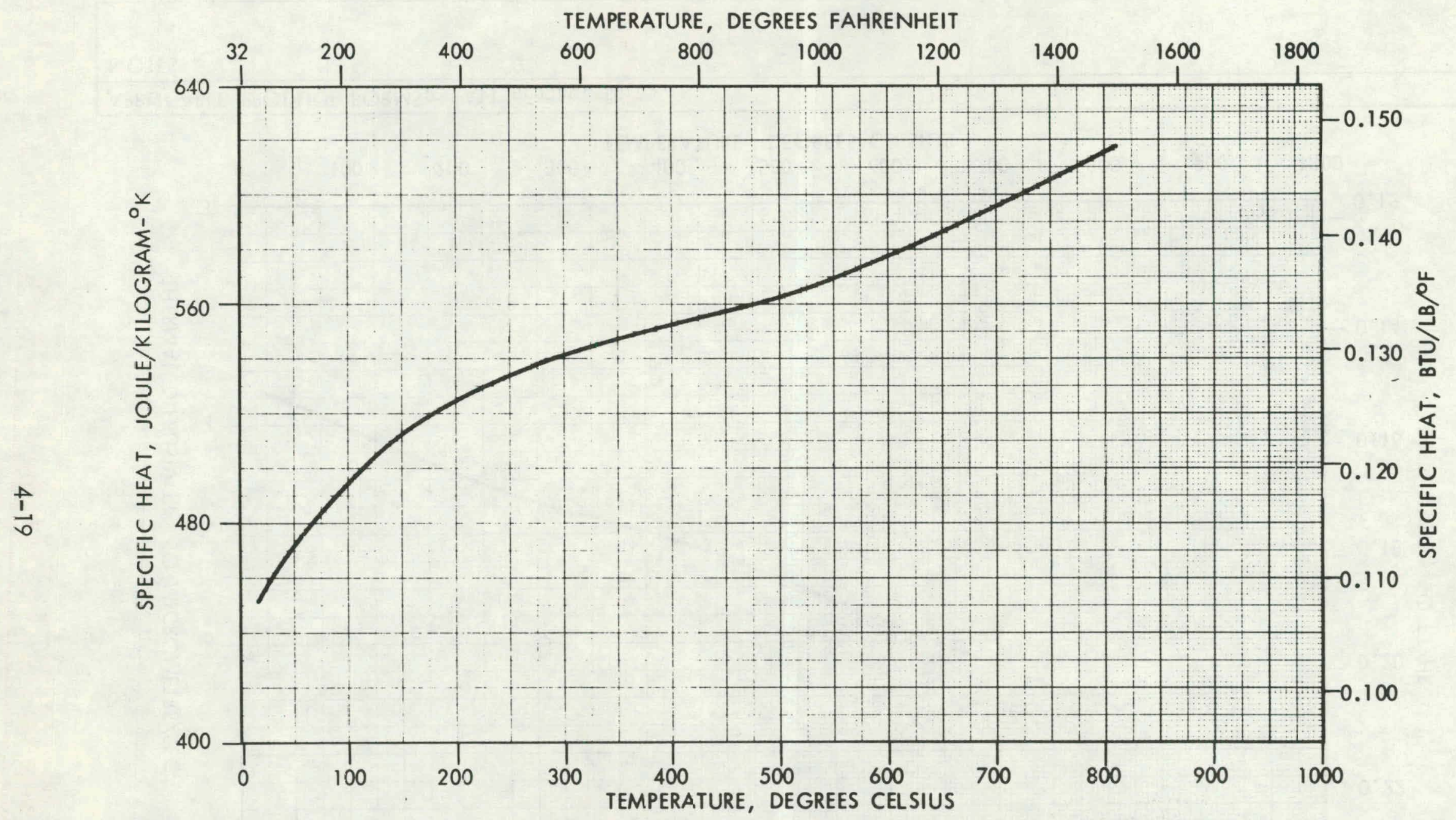

APPLICABLE PRODUCT FORMS: A11 product forms. NOTES: 


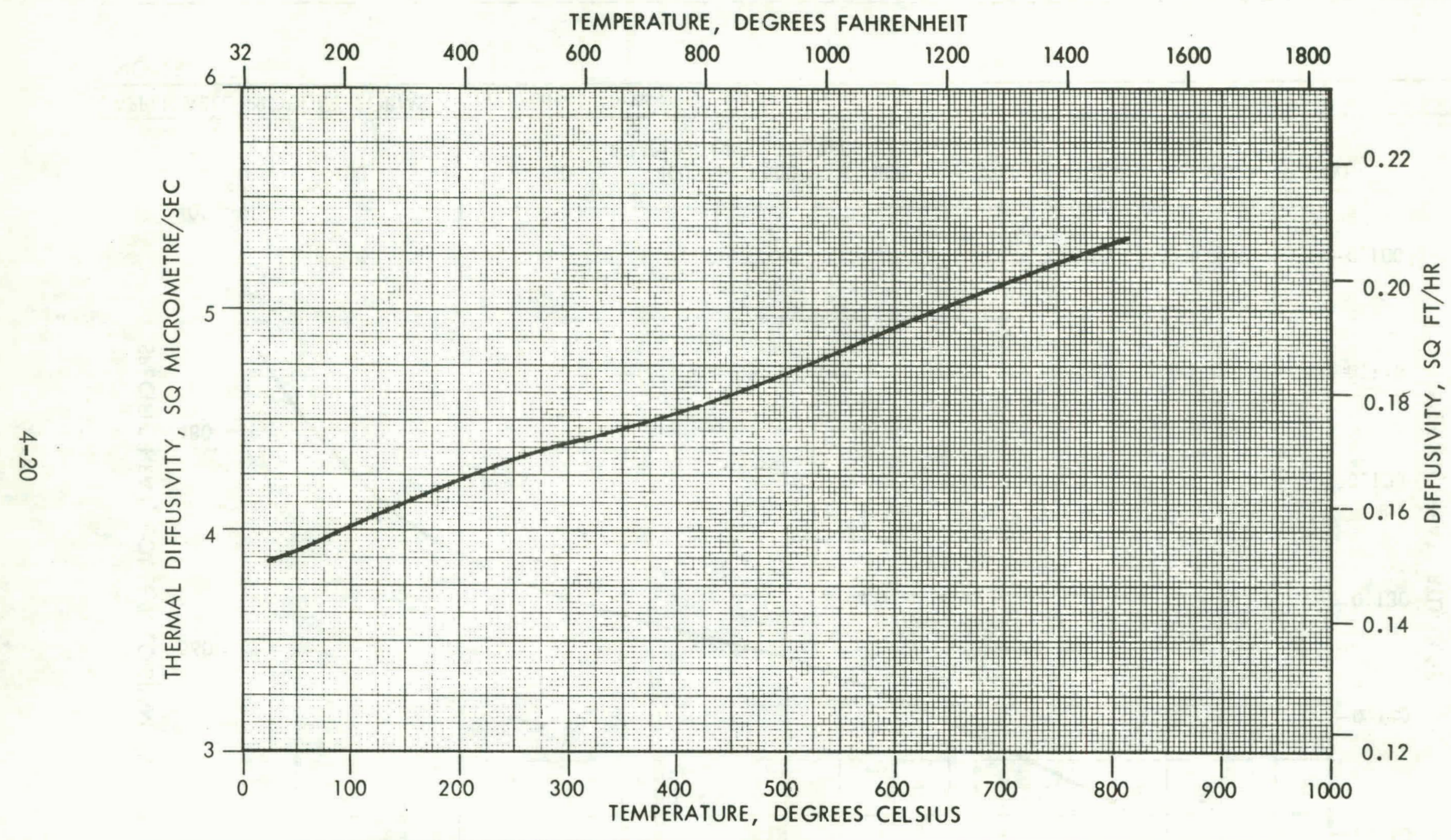

APPLICABLE PRODUCT FORMS: A11 product forms. NOTES:

Figure 3.1-2 Thermal Diffusivity 


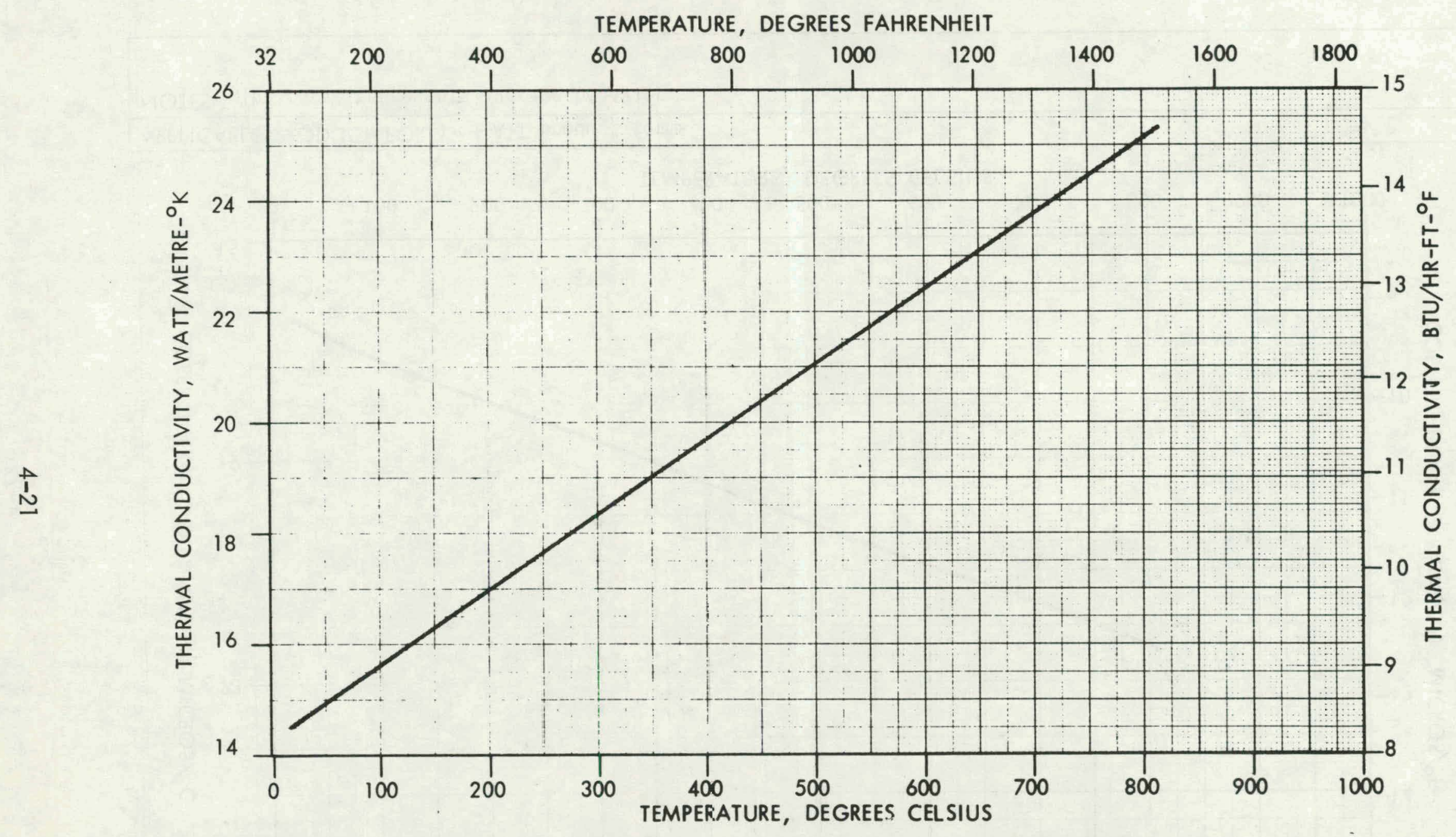

APPLICABLE PRODUCT FORMS: All product forms. NOTES: 


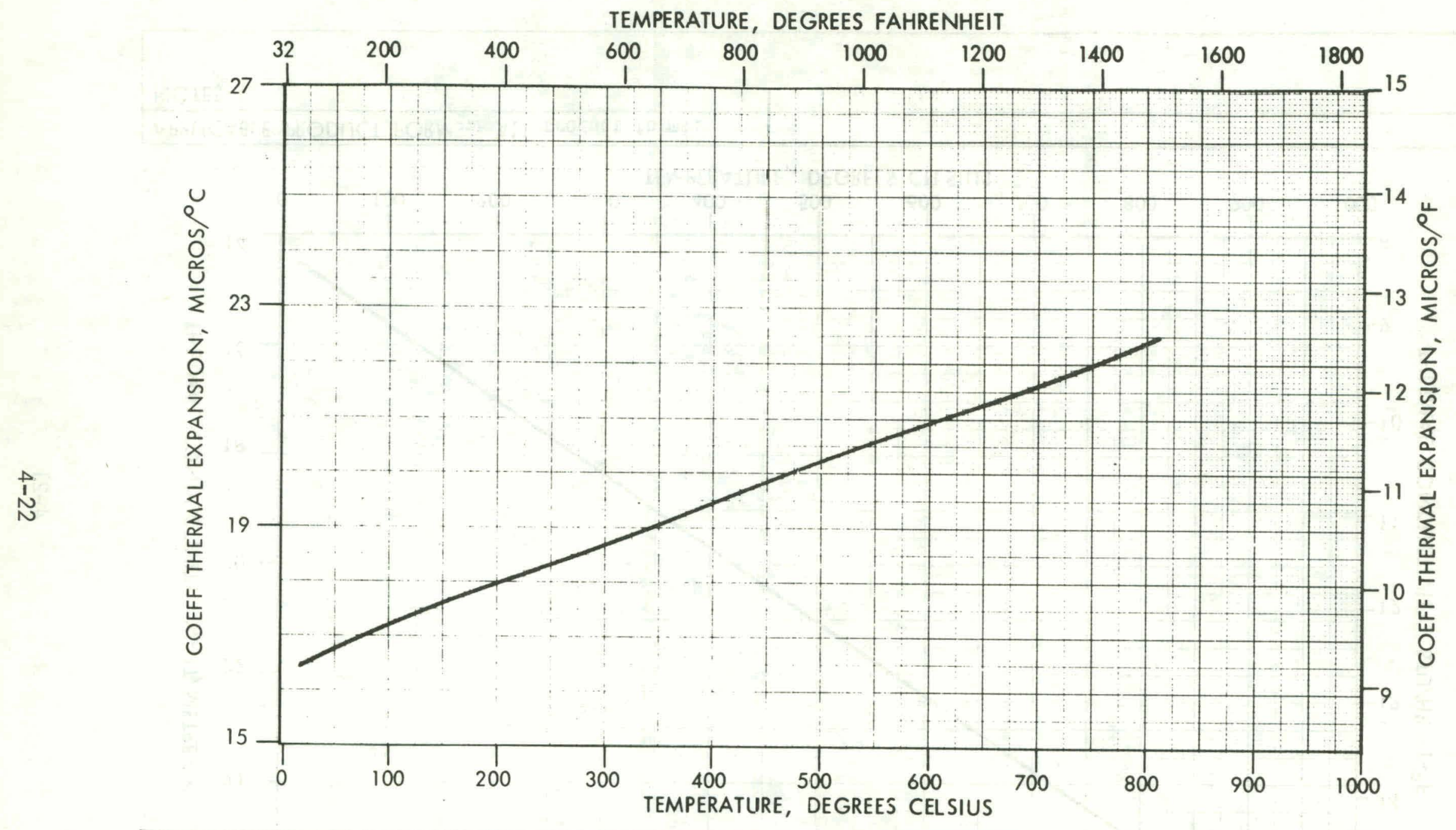

APPLICABLE PRODUCT FORMS: A11 product forms.

NOTES: The curve represents average hehavior. 


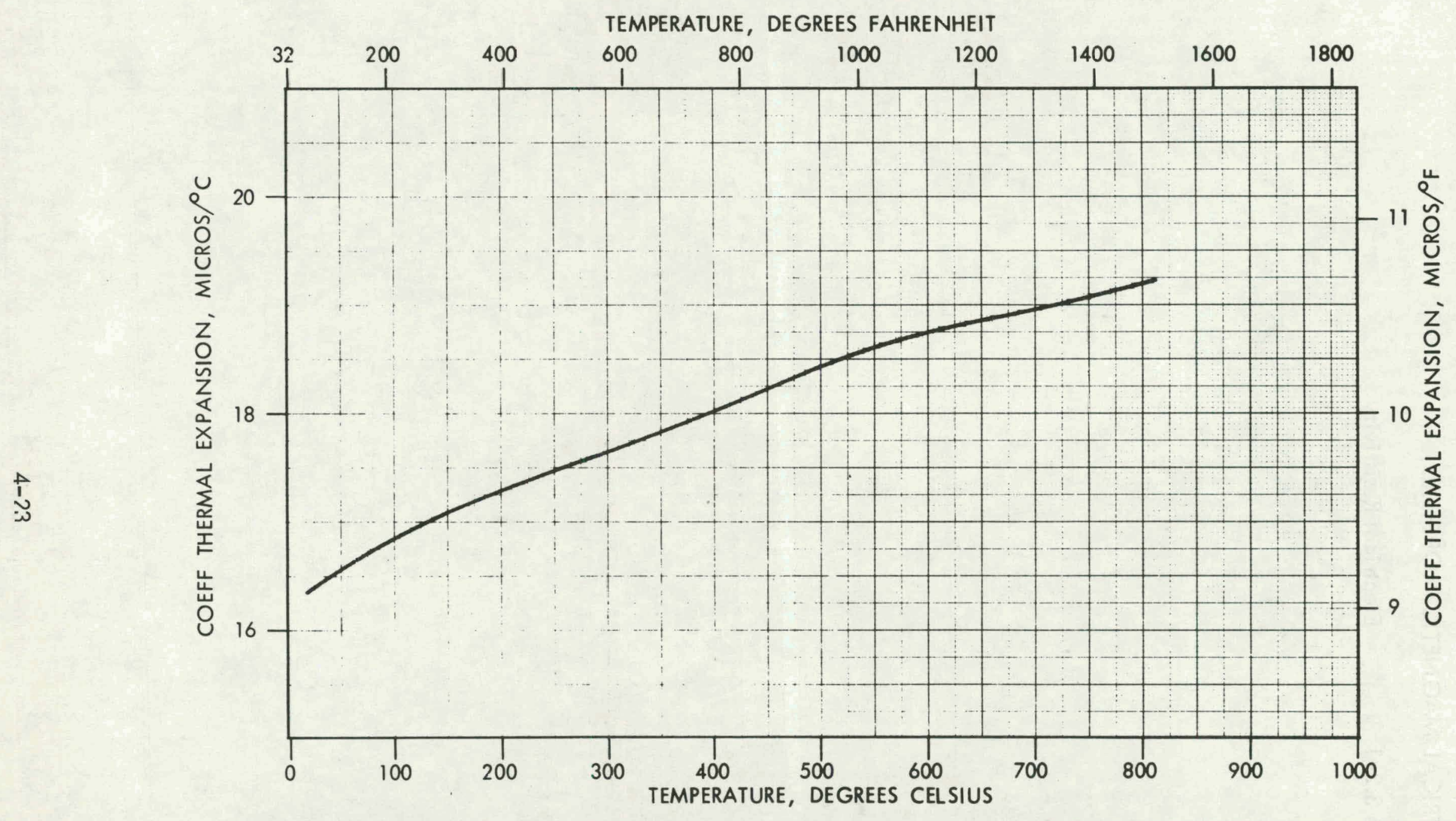

APPLICABLE PRODUCT FORMS: Al1 product forms .

NOTES. The curve represents average behavior. 
3.2 ELECTRICALMAGNETIC PROPERTIES

Figure 3.2 Electrical Resistivity 


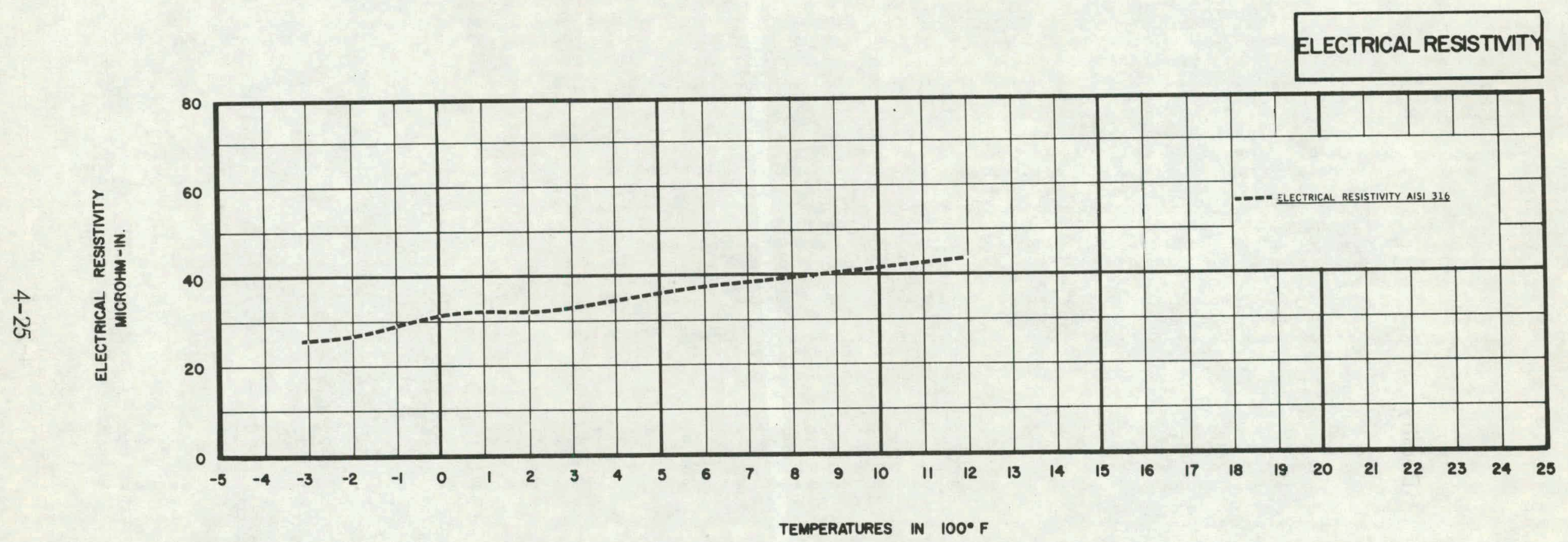

Figure 3.2-1 Electrical Resistivity 


\subsection{MISCELLANEOUS}

Figure 3.3-1

Density 


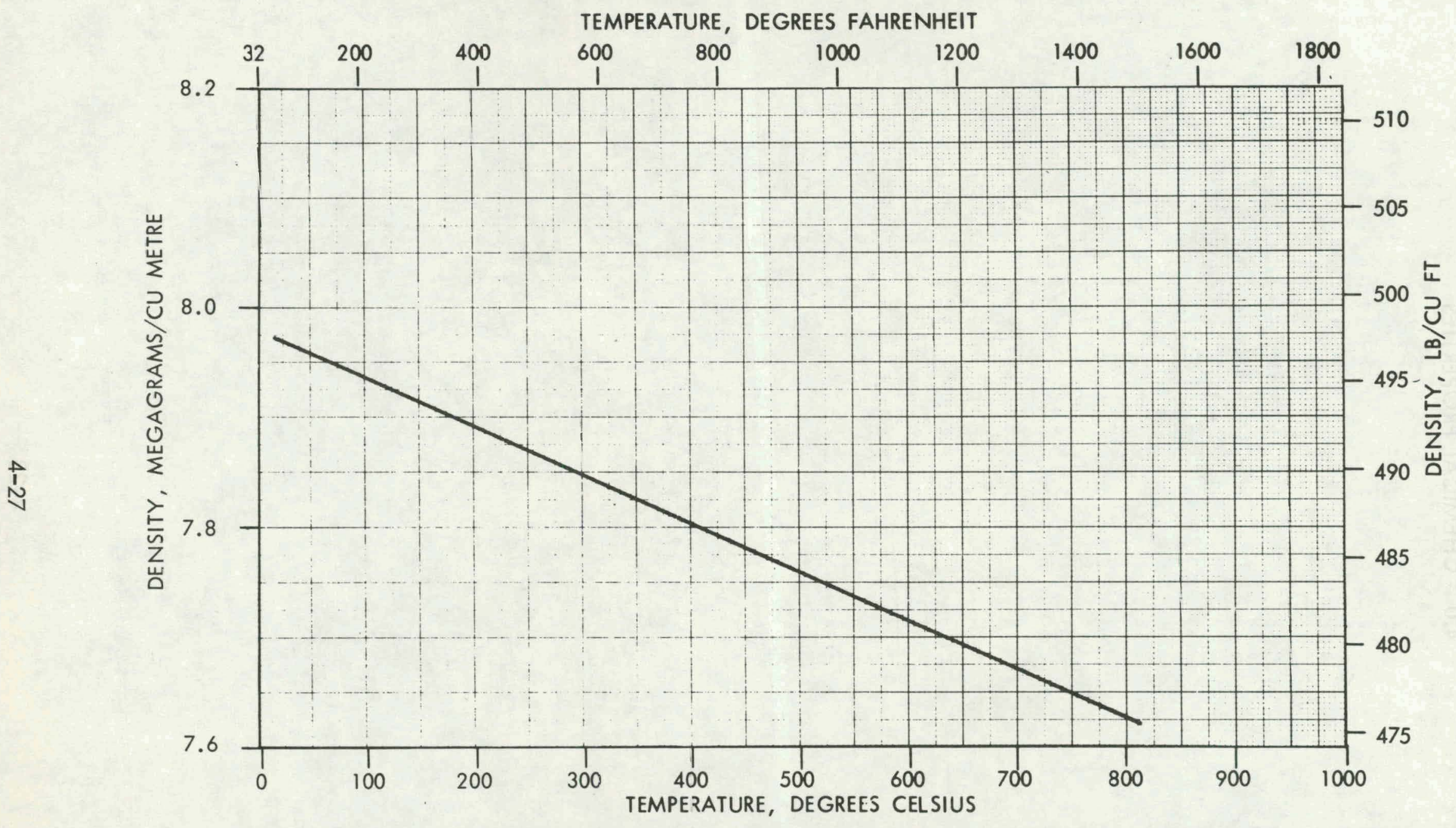

APPLICABLE PRODUCT FORMS: A11 product forms. NOTES: 


\subsection{CHEMICAL PROPERTIES}


5.0 NUCLEAR PROPERTIES 


\subsection{REFERENCES}

6.1 Simmons, W. F. and Cross, H. C. "The Elevated Temperature Properties of Stainless Steels," ASTM STP-124, 1952.

6.2 Simmons, W. F. and Van Echo, J. A., "The Elevated Temperature Properties of Stainless Steels," ASTM DS5-SI, 1965.

6.3 Aerospace Structural Metals Handbook, Syracuse University Institute, AFSC Project 7381, Contract AF 33 (616) - 7792, through Supplement of March 1964.

6.4 Lucks, C. F. and Deem, H. W., "Thermal Properties of Thirteen Metals," ASTM Special Technical Publication No. 227, 1958.

6.5 R. L. Lowell, K. A. Warren, et al, Cryogenic Properties of Reactor Materials, unpublished report, NBS.

6.6 Mechanical and Physical Properties of the Austenitic Chromium-Nickel Stainless Steels at Sub-Zero Temperatures.

6.7 "Piping Design Guide for LMFBR Sodium Piping," SAN-78I-I, C. F. Braun, February I, 1971. 
SECTION 5.0

NITRONIC 33 (ARMCO 18-3 Mn STAINLESS STEEL) 


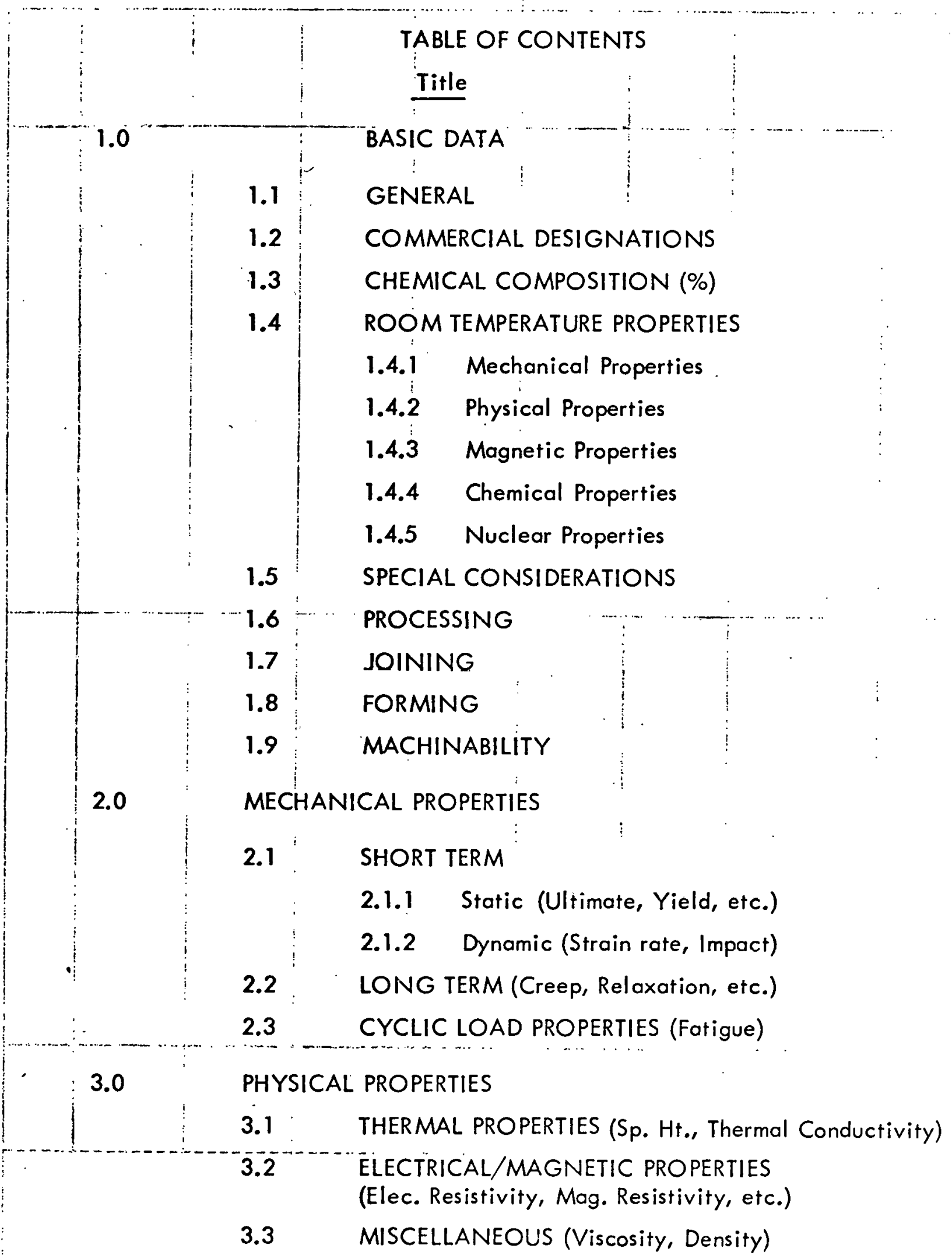


4.0 CHEMICAL PROPERTIES

5.0 NUCLEAR PROPERTIES

6.0 REFERENCES 


\subsection{BASIC DATA}

\subsection{GENERAL INFORMATION}

Nitronic 33 (Armco 18-3 Mn) is a low-nickel austenitic stainless steel that is better than Type 304 stainless steel in at least five specific areas: (1) Yield strength in the annealed condition is approximately twice that of Type-304; (2) Resistance to stress corrosion cracking is better than Type $304 ;$ (3) Low magnetic permeability is retained even after severe cold working; (4) Strength and ductility at cryogenic temperatures are excellent; and (5) Resistance to wear and galling is much superior to the standard austen itic sta inless steels.

\subsection{COMMERCIAL DESIGNATION}

Armco Nitronic 33 is the new name for Armco 18-3 Mn stainless steel.

Armco 18-3 Mn plate, sheet and strip ore listed in ASTM Specifications A 240 and A 412 (denoted as Grade XM 29). Application for ASTM specification coverage for Armco 18-3 Mn tubing is currently being processed.

\subsubsection{Available Forms}

Armco 18-3 Mn Stainless Steel is available in sheet, strip, plate, tubing and extrusions. All products are supplied to standard AISI tolerances.

$\%$

Carbon Manganese

Phosphorus

Sulfur
0.05 max.

$11.50 / 14.50$

0.060 max.

0.030 max.
$\%$

Silicon

Chromium

Nickel

1.00 max. $17.00 / 19.00$

$2.25 / 3.75$

Nitrogen

$0.20 / 0.40$ 
1.4 ROOM TEMPERATURE PROPERTIES (MIN. ACCEPTABLE)

1.4.1 Mechanical Properties

\begin{tabular}{|c|c|}
\hline$\frac{\frac{\text { Sheet }}{\text { Tensile Ultimate }} \stackrel{\text { Plate }}{\text { Strength }}}{105,000(723) 100,000(689)}$ & Psi (M Pa) \\
\hline$\frac{\text { Tensile Yield Strength }}{55,000(379) \quad 55,000}(0.2 \%)$ & Psi (M Pa) \\
\hline Elongation & Percent \\
\hline
\end{tabular}

Reduction of Area

Percent

Modulus of Elasticity

$\underline{10^{\mathrm{C}} \mathrm{Psi}}$

Hordness

100 max. 100 max.

Rockwell B

1.4.2 Physical Properties

\begin{tabular}{lcc} 
Density at $75^{\circ} \mathrm{F}$ & $\rho$ & $\mathrm{lb} / \mathrm{in}^{3}\left(\mathrm{~g} / \mathrm{cm}^{3}\right)$ \\
\hline
\end{tabular}

Specific Heat

c $\quad \mathrm{BTU} / \mathrm{H}-{ }^{\circ} \mathrm{F}$ 


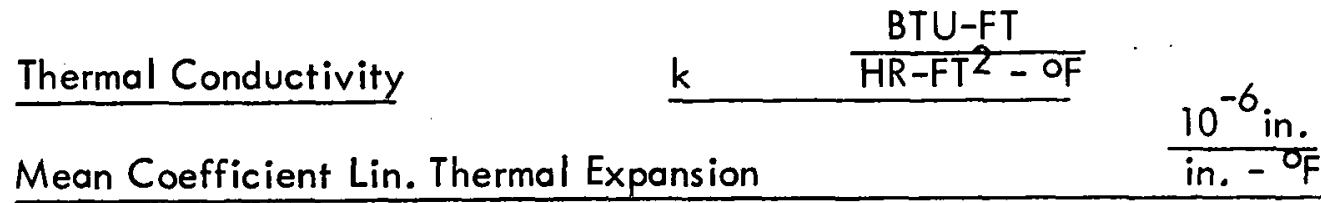

Electrical Resistivity

$\frac{R \quad \text { Microhm }-\mathrm{cm}}{70}$

\subsubsection{Magnetic Properties}

Armco 18-3 Mn Stainless Steel provides very low magnetic permeability even after severe cold working. When cold reduced as much as $70 \%$, its permeability remains below 1.02 .

TABLE VII

MAGNETIC PERMEABIL ITY

ASTM A 324-64, METHOD 4

\begin{tabular}{|c|c|c|}
\hline \% Cold Reduction & $\mathrm{H}=500$ & $\mathrm{H}=1000$ \\
\hline 0 & 1.0014 & 1.0013 \\
10 & 1.0013 & 1.0012 \\
20 & 1.0015 & 1.0011 \\
30 & 1.0010 & 1.0013 \\
40 & 1.0015 & 1.0013 \\
50 & 1.0011 & 1.0012 \\
60 & 1.0012 & 1.0012 \\
70 & 1.009 & 1.0013 \\
\hline
\end{tabular}


1.4.4 Chemical Properties

1.4.5 Nuclear Properties

1.5 SPECIAL CONSIDERATIONS

"!

1.6 PROCESSING 
1.7 JOINING

See reference 6.4.

1.8 FORMING

1.9 MACHINABILITY 
2.0 MECHANICAL PROPERTIES

\section{1 SHORT TERM}

Figure 2.1-1 Typical Stress-Strain Curve

Table 2.1-1 Short Time Elevated-Temperature Properties

3.0 PHYSICAL PROPERTIES

3.1 THERMAL PROPERTIES

Table 3.1-1 Thermal Conductivity

Table 3.1-2 Thermal Contraction

Table 3.1-3 Thermal Expansion

3.2 ELECTRICAL/MAGNETIC PROPERTIES

Table 3.2-1 Electrical Resistivity of Elevated Temperature

3,3 MISCELLANEOUS 
Typical engineering stress-strain curves for Armco 18-3 Mn and Type 304 bar stock (tested in tension) are shown in Figure 2.1-1. The modulus of elasticity (Young's Modulus) of $18-3 \mathrm{Mn}$ is $28.8 \times 10^{6} \mathrm{psi}\left(0.199 \times 10^{6} \mathrm{MPa}\right)$, and Poisson's ratio is 0.295 , both measured at room temperature.

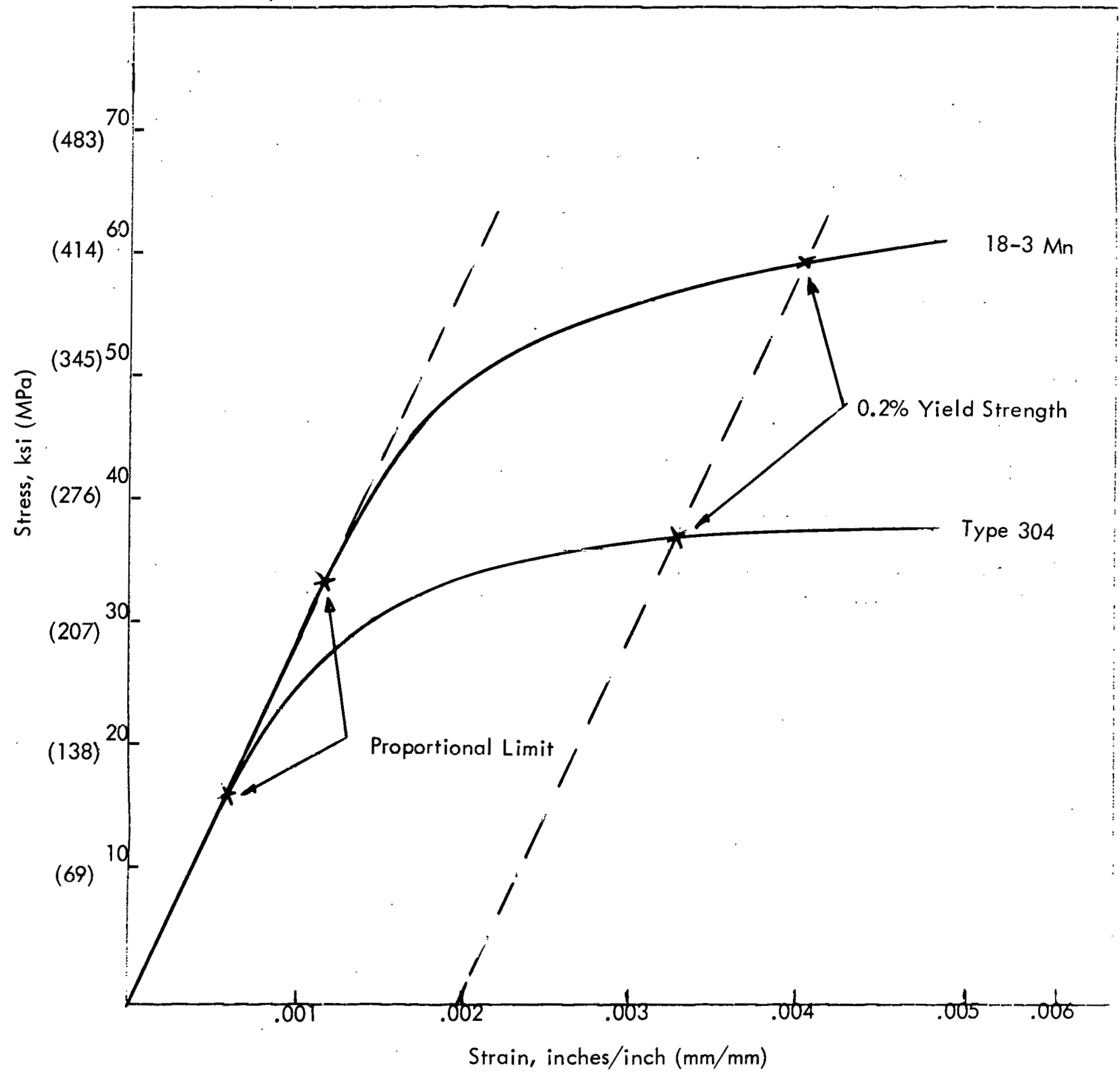

Figure 2.1-1 - Typical Engineering Stress-Strain Curves Obtained in Tension on Ear Samples (Longitudinal Direction) 
TABLE 2.1-1

SHORT-TIME ELEVATED-TEMPERATURE PROPERTIES*

\begin{tabular}{|cc|c|c|c|}
\hline \multicolumn{2}{|c|}{$\begin{array}{c}\text { Temperature } \\
F(C)\end{array}$} & $\begin{array}{c}\text { UTS } \\
\text { ksi }(\mathrm{MPa})\end{array}$ & $\begin{array}{c}0.2 \% \mathrm{YS} \\
\mathrm{ksi}(\mathrm{MPa})\end{array}$ & $\begin{array}{c}\text { Elong } \\
\text { \% in 2" }(50 \mathrm{~mm})\end{array}$ \\
\hline 75 & $(24)$ & $117(807)$ & $72(496)$ & 49 \\
200 & $(93)$ & $106(731)$ & $59(407)$ & 40 \\
400 & $(204)$ & $93(641)$ & $45(310)$ & 44 \\
600 & $(315)$ & $91(627)$ & $39(269)$ & 49 \\
800 & $(426)$ & $82(565)$ & $36(248)$ & 39 \\
\hline
\end{tabular}

Data represent average of eight tests consisting of duplicate longitudinal and transverse tests on each of two heats; both are $0.050 "(1.25 \mathrm{~mm})$ thick annealed sheet samples. 


\subsection{PHYSICAL PROPERTIES}

\begin{tabular}{|ccc|}
\hline \multicolumn{3}{|c|}{ MAGNETIC PERMEABILITY OF NITRONIC 33 } \\
\hline & \multicolumn{2}{c|}{ Permeability } \\
\hline Cold Reduction \% & $\frac{H=500}{1.0014}$ & $\frac{H=1000}{1.0013}$ \\
\hline 0 & 1.0013 & 1.0012 \\
10 & 1.0015 & 1.0011 \\
20 & 1.0010 & 1.0013 \\
30 & 1.0015 & 1.0013 \\
40 & 1.0011 & 1.0012 \\
50 & 1.0012 & 1.0012 \\
60 & 1.0009 & 1.0013 \\
70 &. & \\
\hline
\end{tabular}

3.1 THERMAL PROPERTIES

3.2 ELECTRICAL/MAGNETIC PROPERTIES

3.3 MISCELLANEOUS 
TABLE 3.1-1

THERMAL CONDUCTIVITY AT ELEVATED TEMPERATURES

\begin{tabular}{|c|c|c|c|}
\hline \multicolumn{2}{|c|}{ Temperature } & \multicolumn{2}{|c|}{ Therma Conductivity } \\
\hline $\bar{F}$ & (C) & $\mathrm{Brv} / \mathrm{hr} / \mathrm{ft}$ & $(\mathrm{W} / \mathrm{m} \mathrm{K})$ \\
\hline 212 & $(100)$ & 110 & $(15.8)$ \\
\hline 392 & (200) & 120 & (17.3) \\
\hline 572 & (300) & 131 & (18.9) \\
\hline 752 & $(400)$ & 141 & (20.4) \\
\hline 932 & (500) & 152 & (21.9) \\
\hline 1112 & (600) & 162 & (23.4) \\
\hline 1292 & (700) & 172 & $(24.8)$ \\
\hline 1472 & $(800)$ & 181 & $(26.1)$ \\
\hline
\end{tabular}

TABLE 3.1-2

THERMAL CONTRACTION AT SUB-ZERO TEMPERATURES

\begin{tabular}{|c|c|c|c|c|c|}
\hline \multicolumn{4}{|c|}{ Temperature Range } & \multicolumn{2}{|c|}{ Coefficient of Linear Contraction } \\
\hline & & (o) & & $L / L$ per 0 & L $\mathrm{L}$ per o C \\
\hline $\begin{array}{l}68 \text { to } \\
68 \text { to } \\
68 \text { to } \\
68 \text { to } \\
68 \text { to } \\
68 \text { to } \\
68 \text { to }\end{array}$ & $\begin{array}{r}0 \\
-50 \\
-100 \\
-150 \\
-200 \\
-250 \\
-300\end{array}$ & $\begin{array}{l}(20 \text { tc } \\
(20 \text { tc } \\
(20 \text { tc } \\
(20 \text { tc } \\
(20 \text { tc } \\
(20 \text { tc } \\
(20 \text { tc }\end{array}$ & $\begin{array}{r}-18) \\
-46) \\
-73) \\
-101) \\
-129) \\
-157) \\
-184)\end{array}$ & $\begin{array}{l}8.33 \times 10^{-6} \\
8.31 \times 10^{-6} \\
8.07 \times 10^{-6} \\
7.61 \times 10^{-6} \\
7.05 \times 10^{-6} \\
6.45 \times 10^{-6} \\
5.86 \times 10^{-6}\end{array}$ & $\begin{array}{l}\left(15.00 \times 10^{-6}\right) \\
\left(14.96 \times 10^{-6}\right) \\
\left(14.53 \times 10^{-6}\right) \\
\left(13.70 \times 10^{-6}\right) \\
\left(12.69 \times 10^{-6}\right) \\
\left(11.61 \times 10^{-6}\right) \\
\left(10.55 \times 10^{-6}\right)\end{array}$ \\
\hline
\end{tabular}


TABLE 3.1-3

THERMAL EXPANSION

\begin{tabular}{|cc|cc|}
\hline & Temperature Range & \multicolumn{2}{|c|}{$\begin{array}{c}\text { Coefficient of } \\
\text { Linear Expansion } \\
\text { in/in/oF }\end{array}$} \\
\hline $78-200$ & $(\mathrm{C})$ & $8.93 \times 10^{-6}$ & $\left(16.07 \times 10^{\circ} \mathrm{O}\right)$ \\
$78-400$ & $(25-93)$ & $9.7 \times 10^{-6}$ & $\left(16.51 \times 10^{-6}\right)$ \\
$78-600$ & $(25-316)$ & $9.68 \times 10^{-6}$ & $\left(17.42 \times 10^{-6}\right)$ \\
$78-800$ & $(25-427)$ & $10.07 \times 10^{-6}$ & $\left(18.13 \times 10^{-6}\right)$ \\
$78-1000$ & $(25-538)$ & $10.43 \times 10^{-6}$ & $\left(18.77 \times 10^{-6}\right)$ \\
$78-1200$ & $(25-649)$ & $10.84 \times 10^{-6}$ & $\left(19.51 \times 10^{-6}\right)$ \\
$78-1400$ & $(25-760)$ & $11.15 \times 10^{-6}$ & $\left(20.07 \times 10^{-6}\right)$ \\
$78-1600$ & $(25-871)$ & $11.40 \times 10^{-6}$ & $\left(20.52 \times 10^{-6}\right)$ \\
$78-1800$ & $(25-981)$ & $11.69 \times 10^{-6}$ & $\left(21.04 \times 10^{-6}\right)$ \\
\hline
\end{tabular}

TABLE 3.2-1

ELECTRICAL RESISTIVITY AT ELEVATED TEMPERATURES

\begin{tabular}{|c|c|c|}
\hline \multicolumn{2}{|c|}{ Temperature } & $\begin{array}{c}\text { Resistivity } \\
\text { Microhm - cm }\end{array}$ \\
\hline 77 & $(25)$ & 74 \\
212 & $(100)$ & 80 \\
392 & $(200)$ & 87 \\
572 & $(300)$ & 94 \\
752 & $(400)$ & 100 \\
932 & $(500)$ & 106 \\
1112 & $(600)$ & 111 \\
1292 & $(700)$ & 115 \\
1472 & $(800)$ & 119 \\
\hline
\end{tabular}


4.0 CHEMICAL PROPERTIES 
5.0 NUCLEAR PROPERTIES

$5-13$ 


\subsection{REFERENCES}

6.1 Armco Steel Corporation, Product Data Bullentin S53, Advanced Materials Division, LA-3173.

6.2 : Alloy Digest

6.3 Austenitic Stainless with Unusual Mechanical and Corrosion Properties, E. E. Denhard and R. H. Espy, Supervisory Research Engineers, Advanced Materials Division, Armco Steel Corporation, (Presented at Petroleum Mechanical Engineering with Underwater Technology Conference, September, 1971 - Houston, Texas.

6.4 Evaluation of Nitronic 33 Stainless Steel for TFTR Device Application, J. M. Wells and M. W. Hagadorn, Westinghouse Research Report 75-8C3-WETWO-R 1, Sept. 1975. 
SECTION 6.0

MOLYBDENUM 


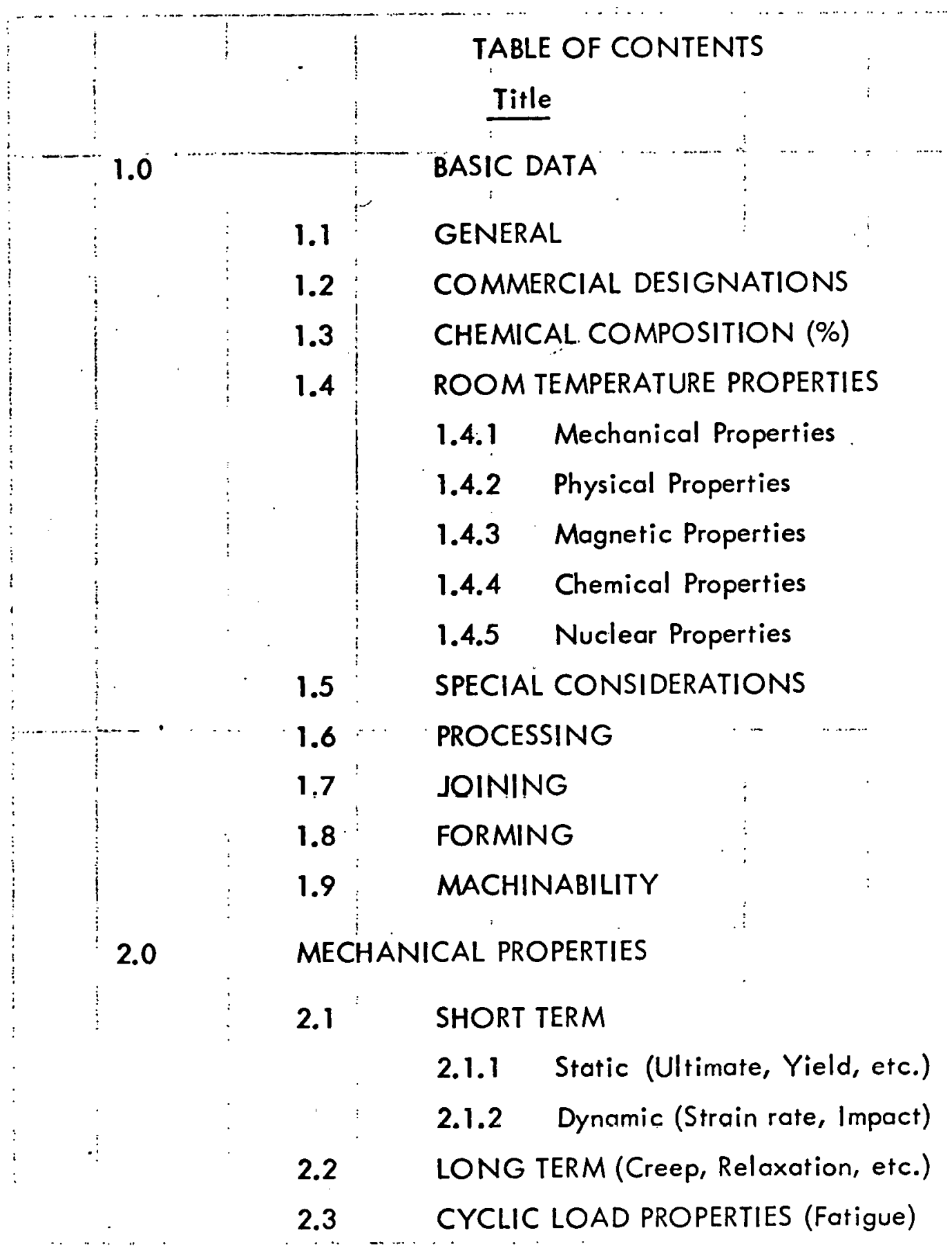

3.0 PHYSICAL PROPERTIES

3.1 THERMAL PROPERTIES (Sp. Ht., Thermal Conductivity)

3.2 ELECTRICAL/MAGNETIC PROPERTIES (Elec. Resistivity, Mag. Resistivity, etc.)

3.3 MISCELLANEOUS (Viscosity, Density) 
4.0 CHEMICAL PROPERTIES

5.0 NUCLEAR PROPERTIES

6.0 REFERENCES 


\subsection{BASIC DATA}

\section{$1.1 \quad$ GENERAL}

Molybdenum is a body-centered-cubic refractory metal possessing sèveral attractive mechanical and thermal properties. Among its advantages are high melting temperature, low density, high modulus of elasticity, excellent strength-to-density ration at elevated temperatures achievable with small alloy additions, and high thermal and electrical conductivity. It has low thermal expansion and relatively low thermal-neutron capture cross-section. It is limited by its poor oxidation resistance at elevated temperatures, poor low temperature ductility, and weld brittleness.

May be made by powder metallurgy techniques and consumable electrode arc casting processes.

\subsection{COMMERCIAL DESIGNATIONS}

Molybdenum, commercially pure.

AVAILABLE FORMS, SPECIFICATIONS, SUPPLIERS

Forms and Condiliurs

Forgings, stress-relieved

Billets

Sheet, Strip, Plate

and Foil

Bar, Rod and Wire

Tube
Specifications

ASTM B -384

ASTM B-385

ASTM B-386

ASTM B-387

WANL-PDS-30032 
1.3 CHEMICAL COMPOSITION (\%)

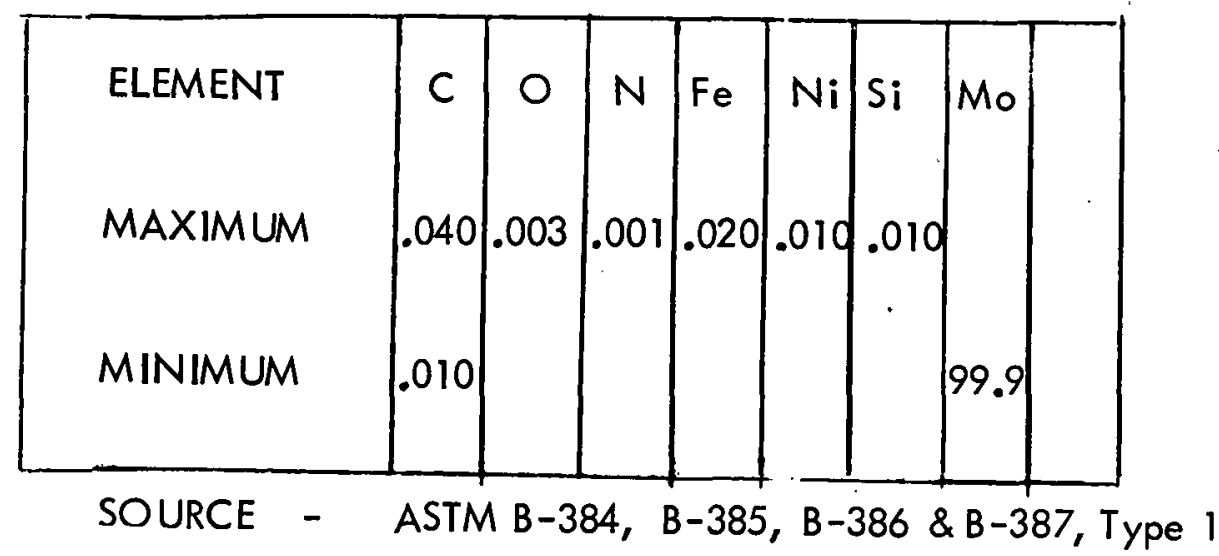

1.4 ROOM TEMPERATURE PROPERTIES

The room temperature properties in Section 1.4 are averages, except for ultimate tensile strength, tensile yield strength, elongation, and reduction of area, which are $80 \%$ of gverage.

\subsubsection{Mechanical Properties}

Ductile-brittle transition temperature is near room temperature for most forms.

\begin{tabular}{cccc}
$\begin{array}{ccc}\text { AS ROLLED } \\
\text { Tensile Ultimate Strength }\end{array}$ & $\begin{array}{c}\text { STRESS } \\
\text { RELIEVED }\end{array}$ & RECRYSTALLIZED \\
\hline Bar 81,000 & 72,000 & 54,000 & PSI \\
Tensile Yield Strength & $F_{\text {TY }}$ & & PSI \\
\hline Bar 63,000 & 66,000 & 44,000 &
\end{tabular}


$\mathrm{F}_{\mathrm{CY}} \longrightarrow \mathrm{PSI}$

$\begin{array}{ccc} & 83,000 & - \\ \text { Ultimate Shear Strength } & F_{\text {SU }} & \\ - & 83,000 & \end{array}$

Shear Yield Strength

FSY

PSI

Ultimate Bearing Strength

$F_{B R U}$

PSI

$\mathrm{e} / \mathrm{D}=1.5$

$\therefore \quad e / D=2.0$.

Yield Bearing Strength

$F_{\text {BRY }}$

PSI

$$
\text { e } / D=1.5
$$

$e / D=2.0$

\section{Elongation}

e

$\%$

32

33

33

Reduction of Area

RA

$\%$

49

55

30

Modulus of Elasticity

E

$10^{6} \cdot \mathrm{PSI}$

Dynamic

46.4

Static

47.2

Comp. Modulus of Elasticity

$\mathrm{E}_{\mathrm{C}}$

$10^{6} \mathrm{PSI}$ 
Modulus of Rigidity

G

$10^{6} \mathrm{PSI}$

Poisson's Ratio

Hardness

Plastic Bending Modulus

\subsubsection{Physical Properties}

\begin{tabular}{lcc} 
Density & $\rho$ & $\mathrm{LB} / \mathrm{IN}^{3}$ \\
\hline $\begin{array}{ll}\text { Specific Heat } \\
\text { Thermal Conductivity }\end{array}$ & $\mathrm{C}$ & $\mathrm{BTU} / \mathrm{LB}-{ }^{\circ} \mathrm{F}$ \\
\hline
\end{tabular}

\section{8}

$\frac{\text { Mean Coeff. Lin. Thermal Expans. a }}{\left(70-212^{\circ} \mathrm{F}\right)}$

Electrical Resistivity $R$ MICROHM - IN.

\section{2}

\subsubsection{Magnetic Properties}

Paramagnetic 
Melting Temperature

$4730^{\circ} \mathrm{F}$

Phase Changes

None

\subsubsection{Chemical Properties}

Compatibility:

Reacts with ozygen; alloys with fungsten, vanadium, titanium, tantalum, columbium, and chromium in solid solution and iron, nickel, cobalt, aluminum, and silicon, forming a second phase of relatively low alloy content.

\section{Corrosion Resistance:}

High resistance to mineral acids and liquid metals, and to water. Corroded by fused alkal is and molten oxidiz ing salts.

\section{Oxidation Resistance:}

$\mathrm{MoO}_{3}$ begins to sublime above $932^{\circ} \mathrm{F}$ and at $1110^{\circ} \mathrm{F}$ volatilization is significant. Existence of molten $\mathrm{MOO}_{3}$ above $1472^{\circ} \mathrm{F}$ leads to catastroph ic oxidation in ordinary atmospheres.

\subsubsection{Nuclear Properties}

Irradiation in general increases tensile yield strength and ductile-brittle transition temperature. 


\subsection{SPECIAL CONSIDERATIONS}

Service temperature maximum is $1000^{\circ} \mathrm{F}$ in oxygen bearing environment.

Must be coated for use in oxidizing atmosphere above $1000^{\circ} \mathrm{F}$.

\subsection{PROCESSING}

\section{Surface Treatment:}

Treated by several methods for oxidation resistance and resistance to gall and wear. Clean and degrease by conventional methods.

\section{Thermal Treatment:}

$$
\begin{aligned}
& \text { Stress relief temperature }=1600-1800^{\circ} \mathrm{F} \\
& \text { Annealing temperature }=1800-2200^{\circ} \mathrm{F}
\end{aligned}
$$

\subsection{JOINING}

Can be welded to itself and any material with which it readily alloys by arc, electric resistance, percussion, flash, or electron beam techniques. Welds are of generally poor quality; cart (weld) zone is normally brittle at room temperature. Can also be brazed and mechanically joined. Arc fusion welds of powder, metallurgical grades, generally are brittle and exhibit weld cracking in heavy section joints.

\subsection{FORMING}

In itial breakdown of the ingot is usually by extruding, with forging at $2150^{\circ}$ to $2350^{\circ} \mathrm{F}$, and fin ishing at $1500^{\circ}$ to $1700^{\circ} \mathrm{F}$.

Can also be formed by spinning, shearing, stamping, punching, hydro forming, bending, and drawing. 


\subsection{MACHINABILITY}

Best with uniform fibrous grain produced with normal amounts of working.

General machinability is comparable to austenitic stainless steels. 


\subsection{MECHANICAL PROPERTIES \\ (As function of temperature)}

\subsection{SHORT TERM}

\subsubsection{Static}

Figure 2.1-1 Tensile Strength

Figure 2.1-2 Impact Strength Rockwell Hardness

Figure 2.1-3 Modulus of Elasticity

Figure 2.1-4 Poisson's Ratio

Figure 2.1-5 Compressive Strength 


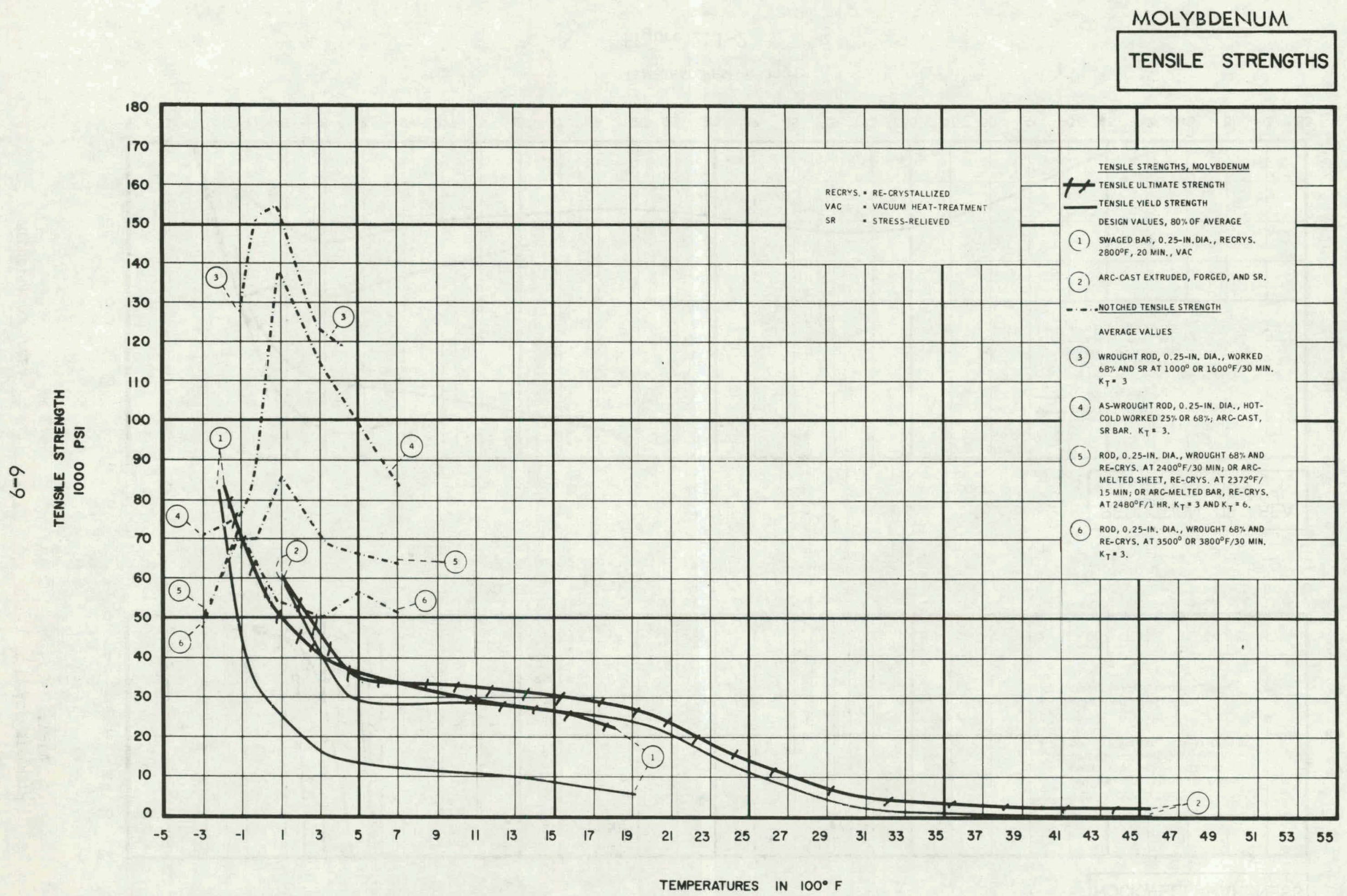

Figure 2.1-1 
MOLYBDENUM
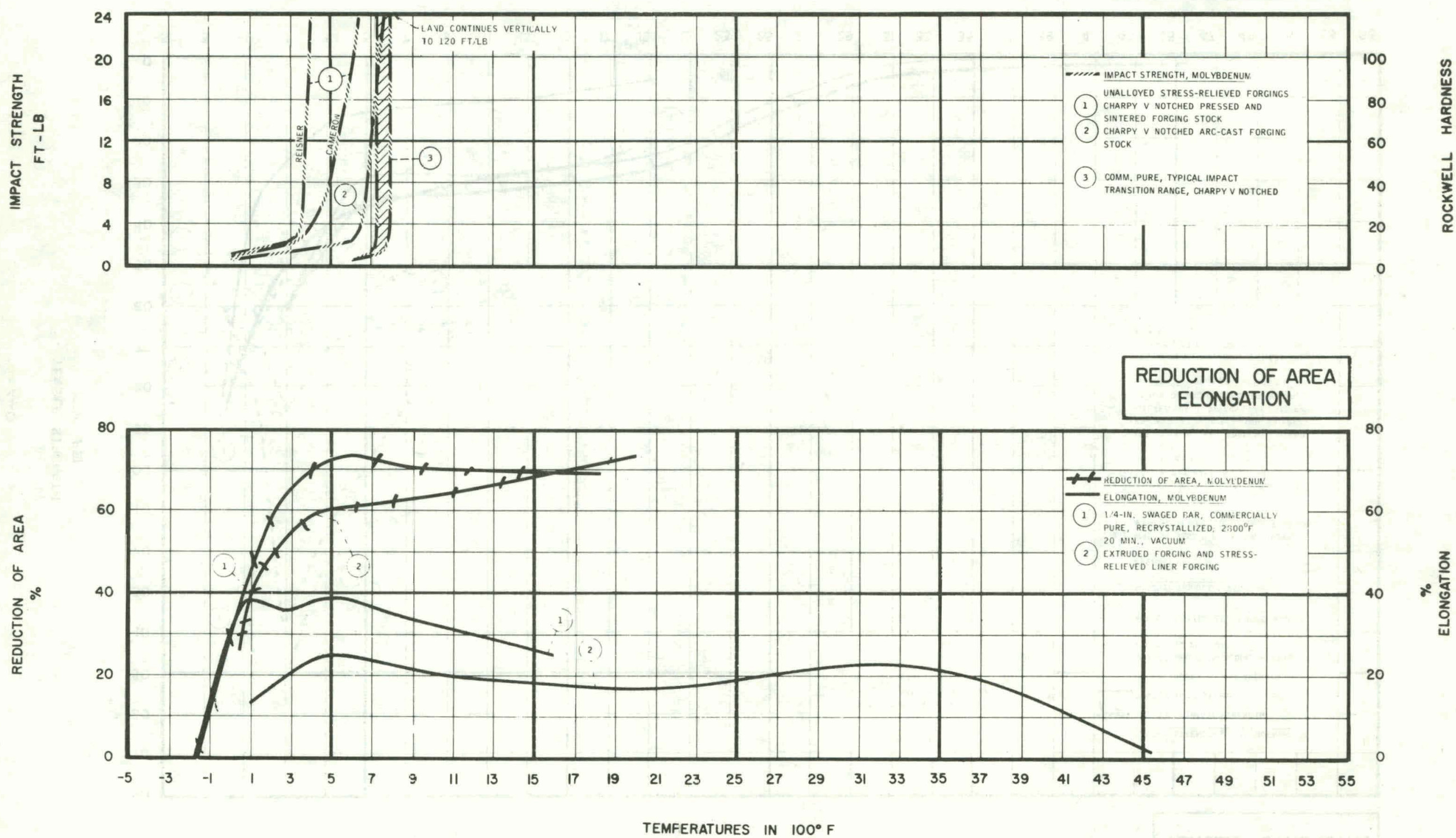

Figure 2.1-2 


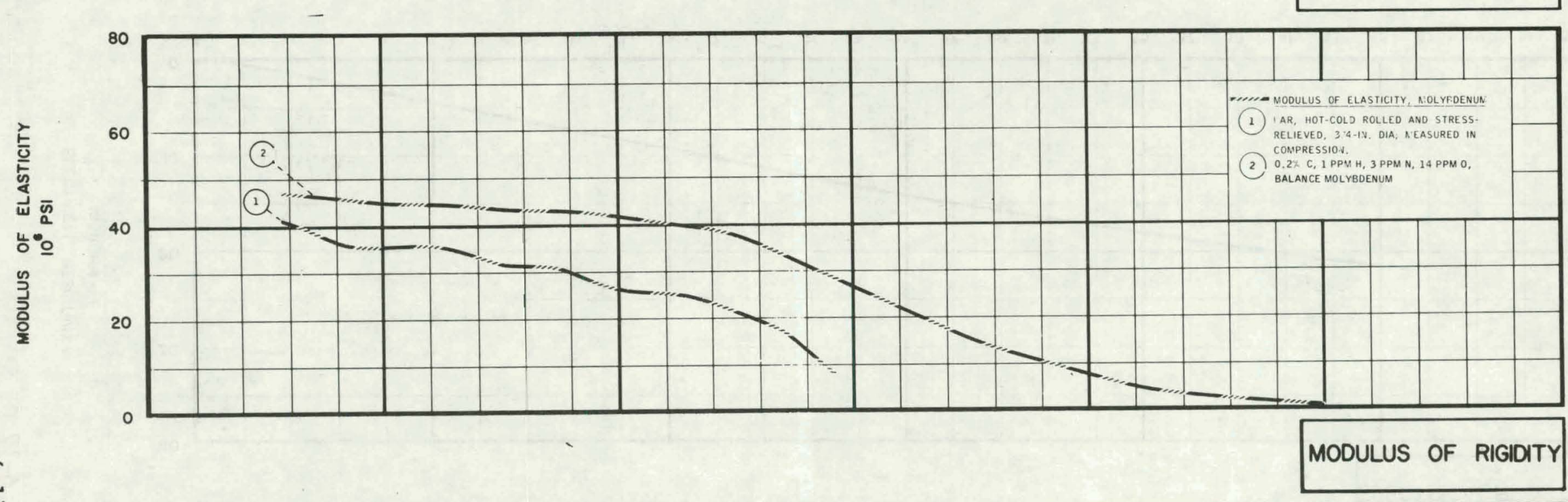

ב

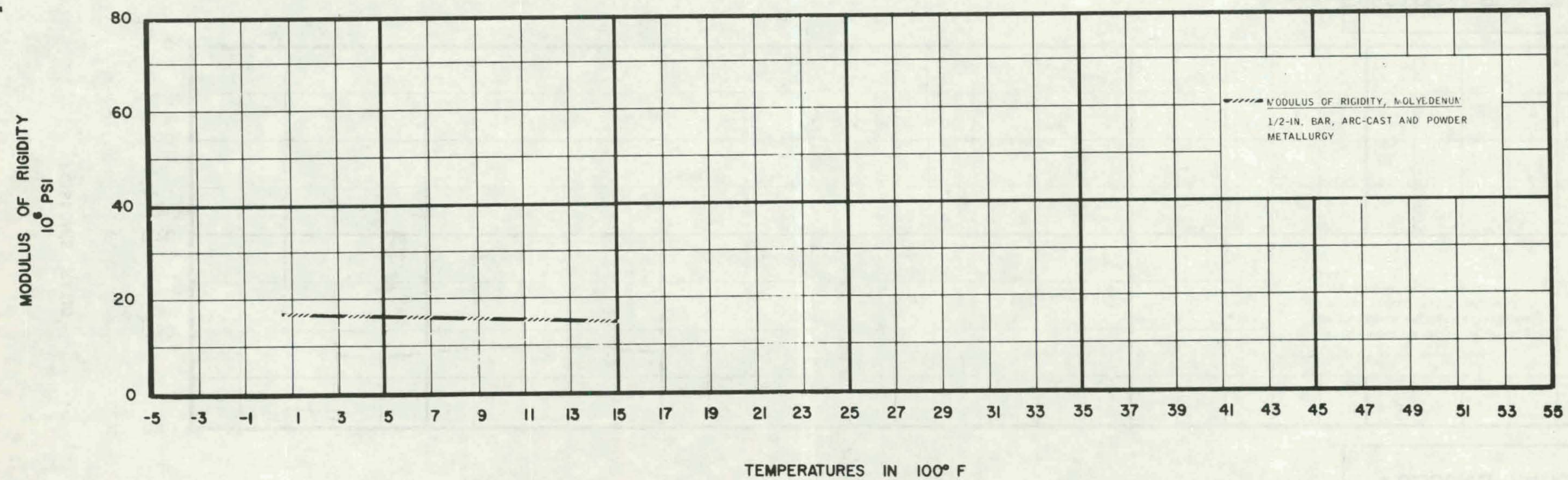

Figure 2. 1-3 


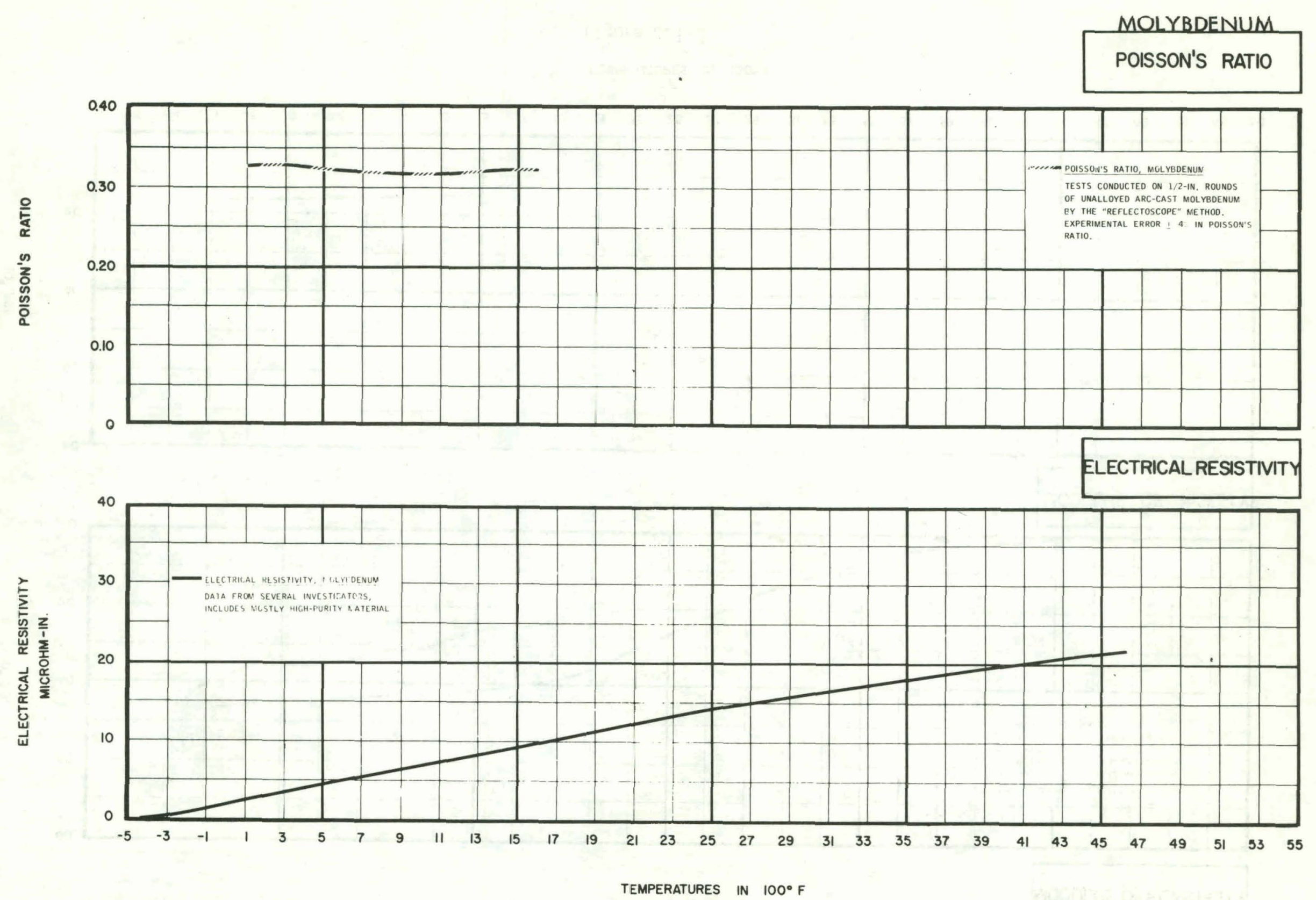

Figure 2.1-4 
MOLYBDENUM

STRENGTHS

COMPRESSIVE, SHEAR, BEARING

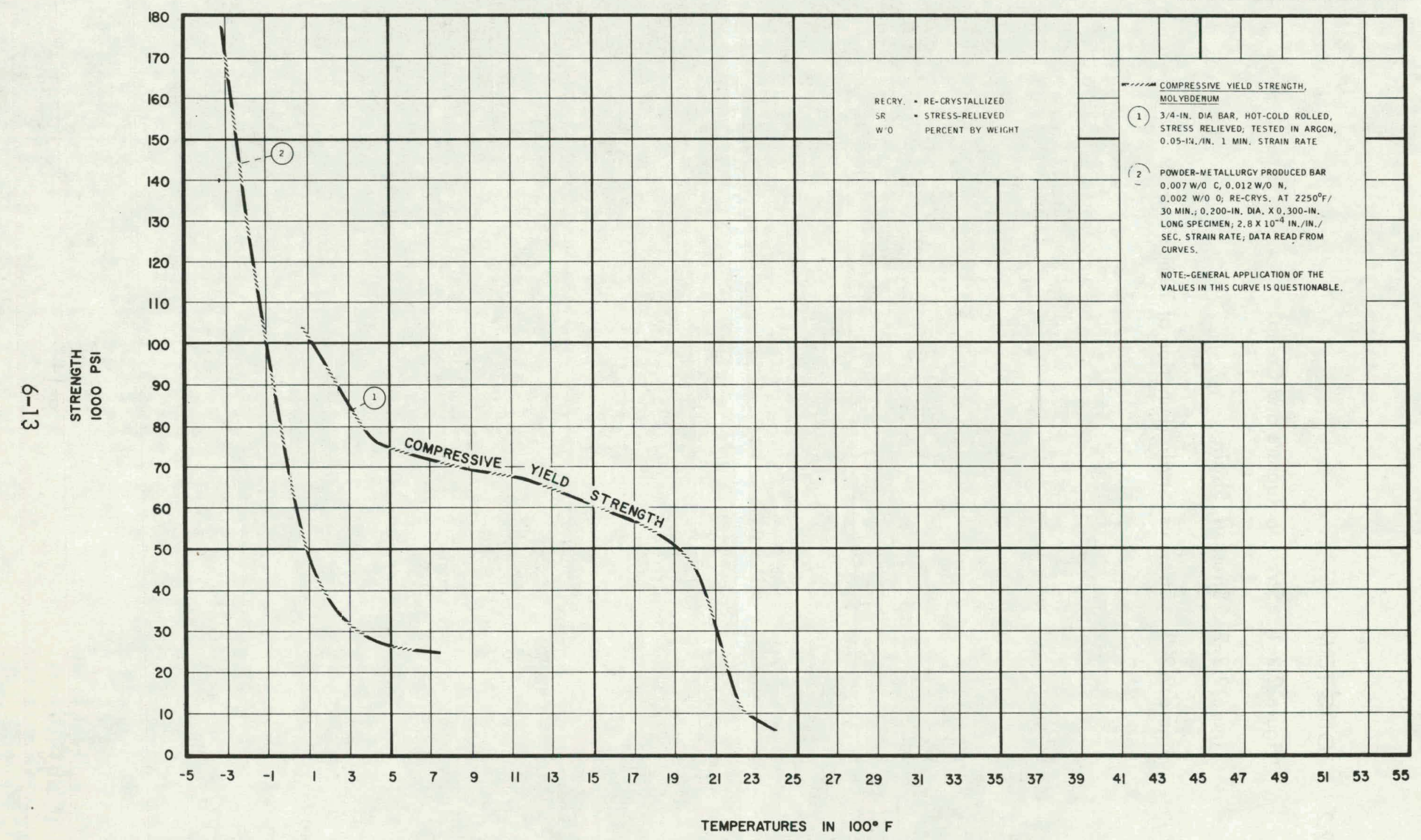

Figure 2.1-5 
2.2 LONG TERM

Figure 2.2-1 Stress to Rupture and Creep

Figure 2.2-2 Stress vs. Strain

Figure 2.2-3 Stress vs. Strain (Function of Temperatures) 


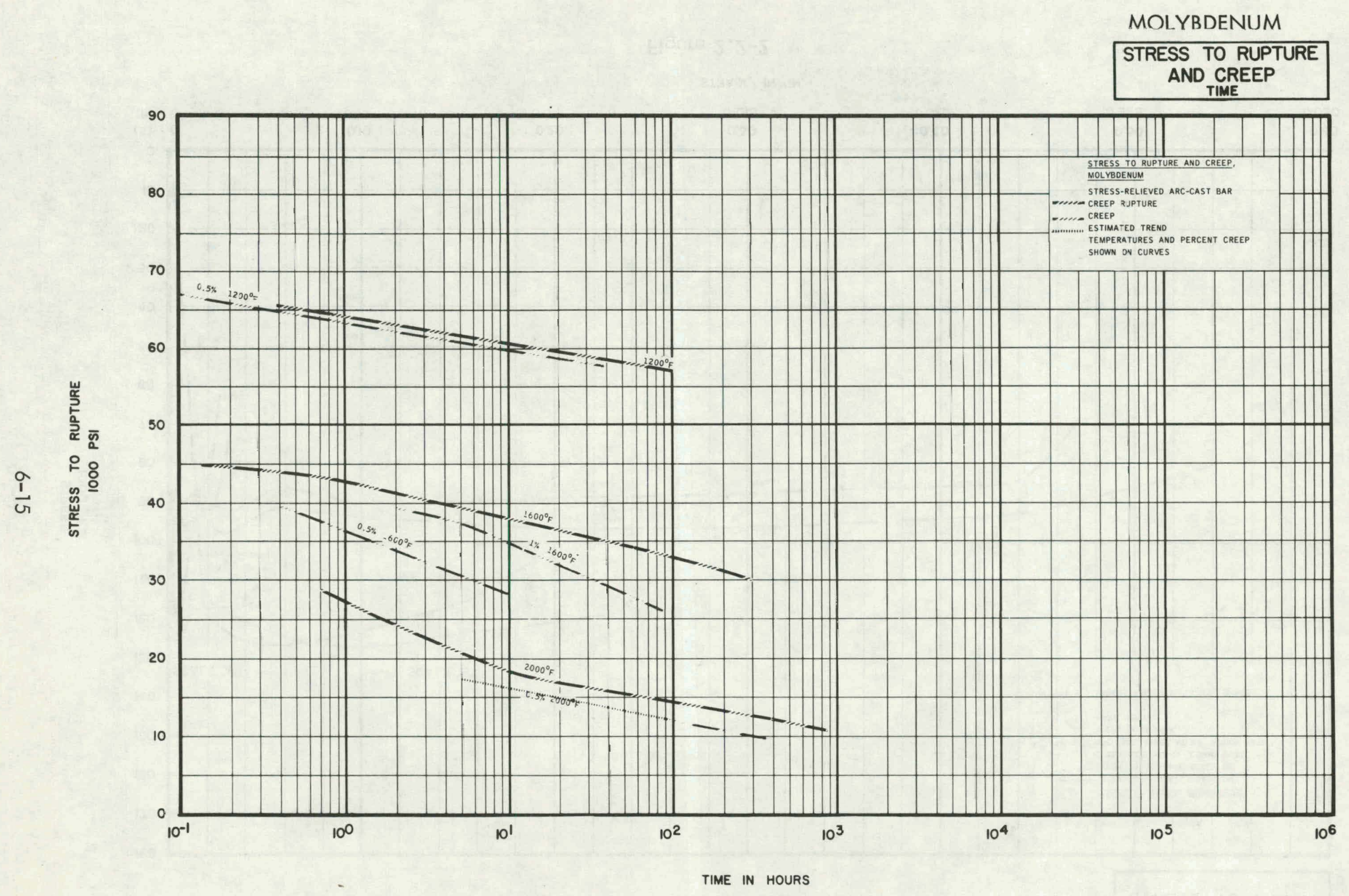

Figure 2.2-1 


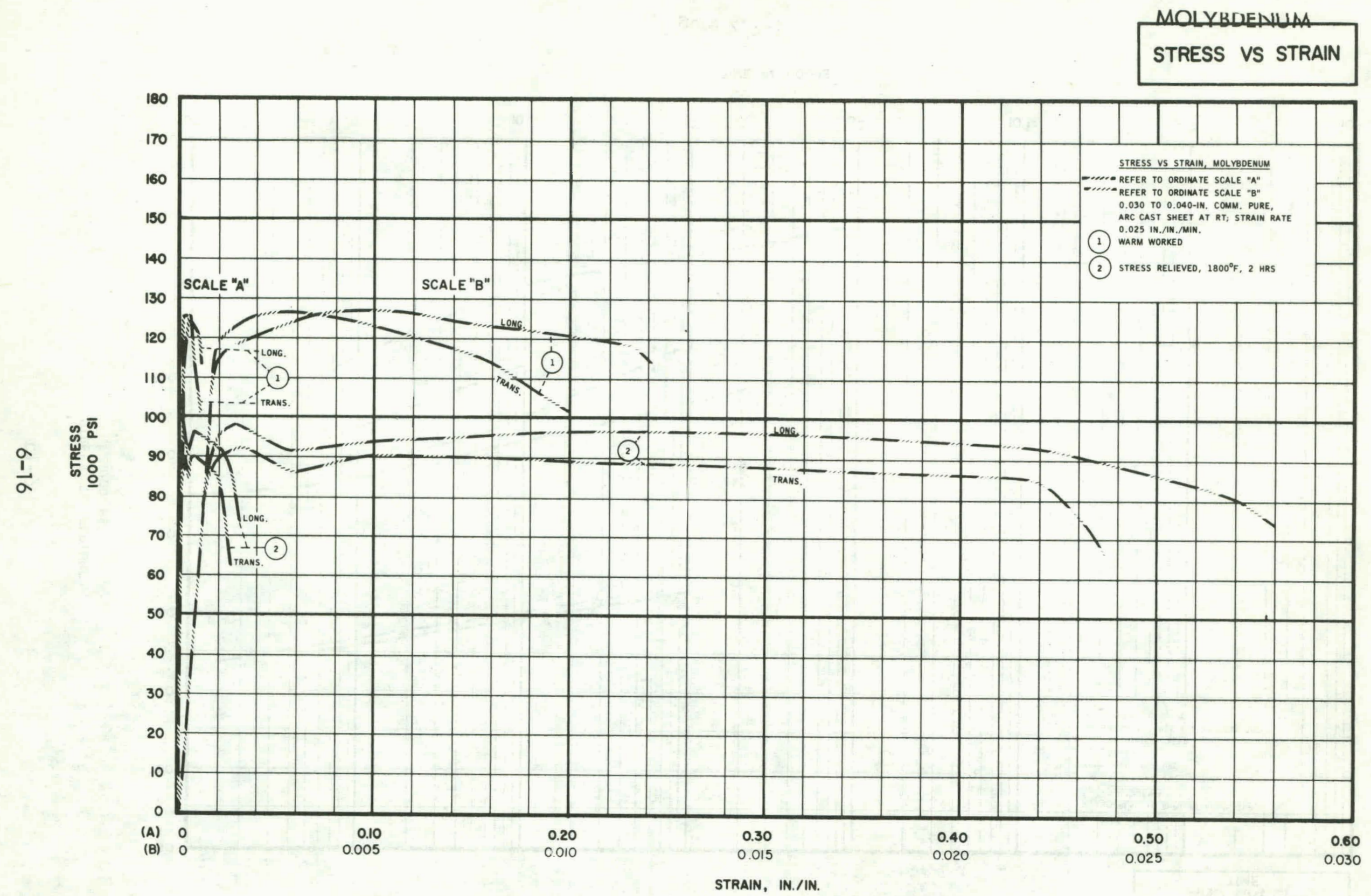

Figure 2.2-2 


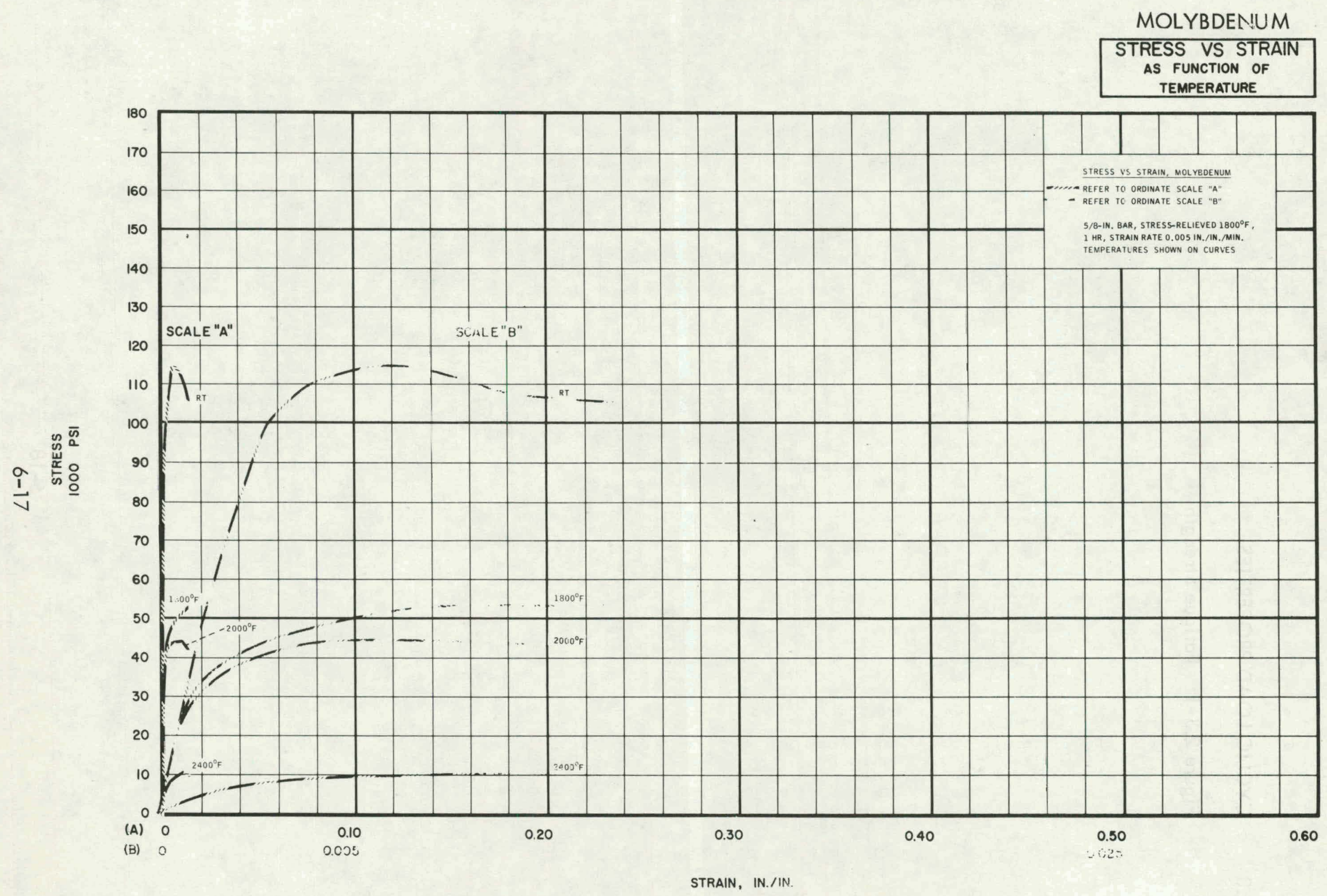

Figure 2.2-3 


\subsection{CYCLIC LOAD PROPERTIES}

Figure 2.3-1 Fatigue Strength 


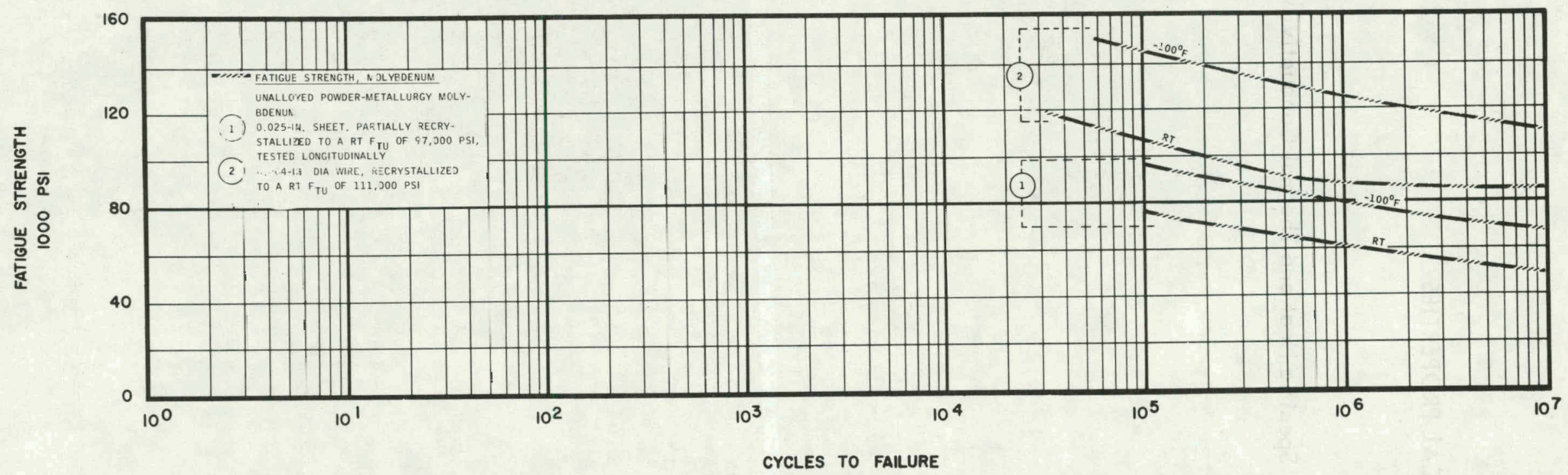

$\frac{a}{0}$

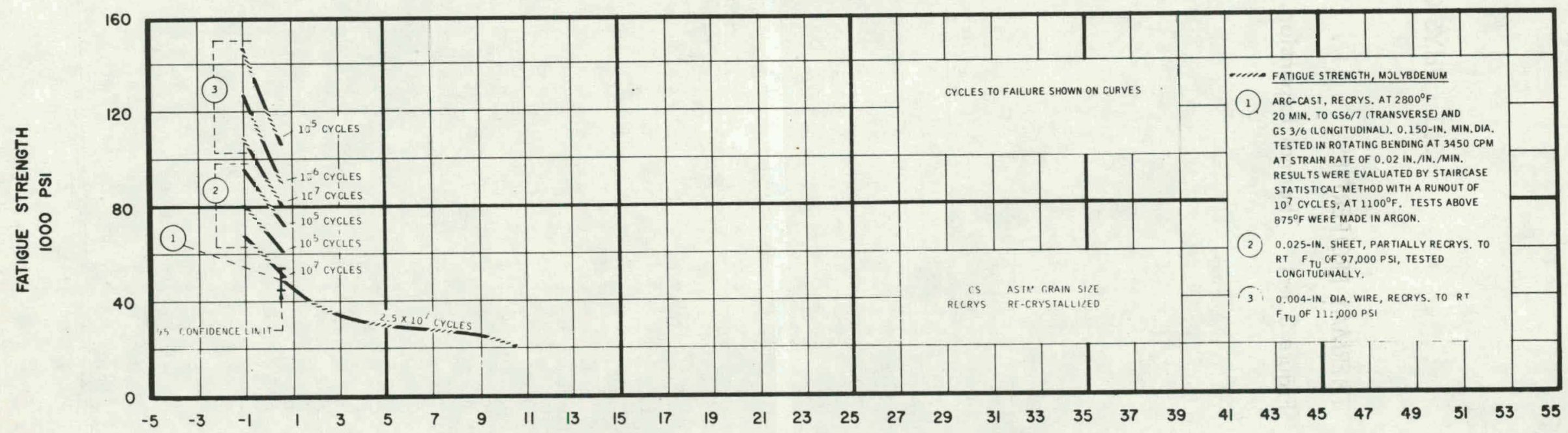

TEMPERATURES IN $100^{\circ} \mathrm{F}$

Figure 2.3-1 


\subsection{PHYSICAL PROPERTIES}

\subsection{THERMAL PROPERTIES}

Figure 3.1-1 Thermal Expansion, Specific Heat and Thermal Conductivity 


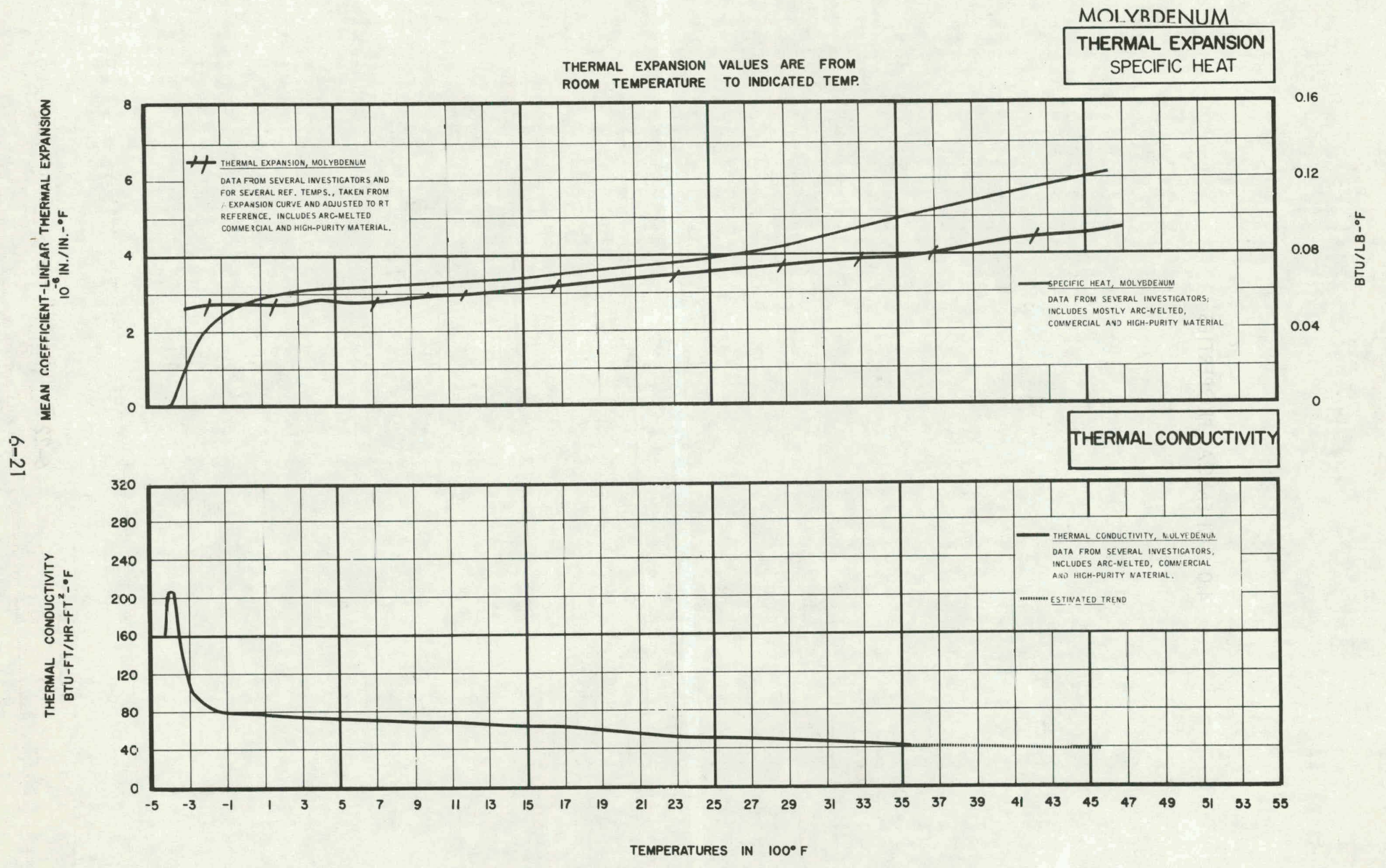

Figure 3.1-1 


\subsection{CHEMICAL PROPERTIES}


5.0 NUCLEAR PROPERTIES 


\subsection{REFERENCES}

6. I Aerospace Structural Metals Handbook, Syracuse University Institute.

6.2 Determination of the Tensile Properties Up to $4500^{\circ} \mathrm{F}$ and the Impact DuctileBrittle Transition Temperature of Unalloyed Molybdenum Forgings - Southern Research Institute Report 5376 - 1364 XII, June 1962.

6.3 Further Investigations of Notch Sensitivity of Refractory Metals, Battelle Report ASD-TDR-62-1004, December 1962.

6.4 Arc-Cast Molybdenum and its Alloys, Universal - Cyclops Steel Corp, 1962.

6.5 The Engineering Properties of Molybdenum and Molybdenum Alloys, DMIC (Report 189, Battelle, September 1963.

6.6 Molybdenum Metal Climax Molybdenum Co, 1960.

6.7 R. B. Stewart and V. J. Johnson (editors) "A Compendium of the Properties of Materials at Low Temperatures, "WADD-TR-60-56, Part 4, Cryogenic Engineering Laboratory, NBS, Boulder, Colo., December 1961.

6.8 A. Goldsmith, et al, Armour Research Foundation, Handbook of Thermophysical Properties of Solid Materials, Vol. 1-5, Rev. ed. NAOC TR-58-476, The Macmillan Co., N. Y., 1960. 
SECTION 7.0

MOLYBDENUM ALLOY - TZM 


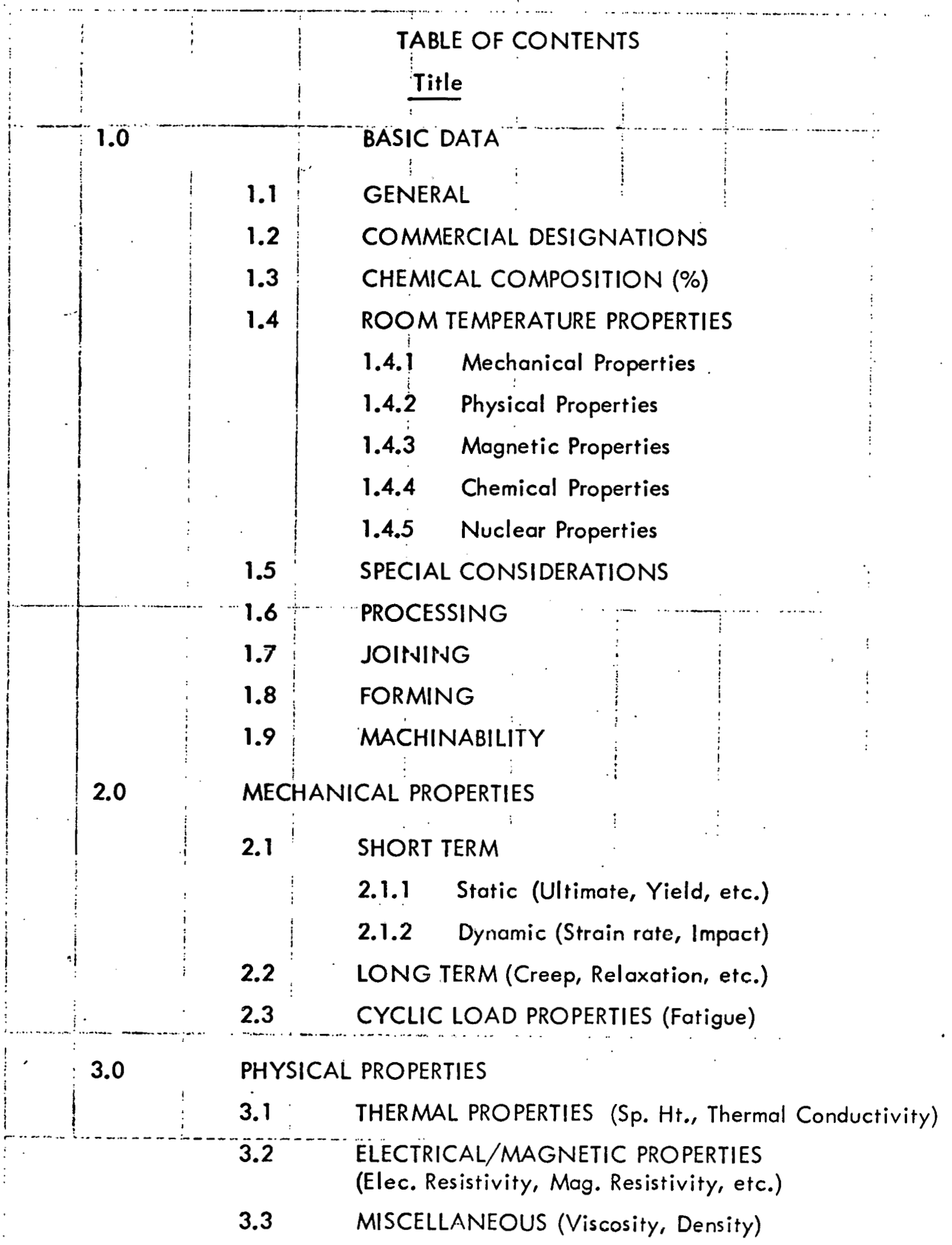




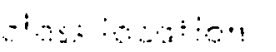

4.0 CHEMICAL PROPERTIES

5.0

NUCLEAR PROPERTIES

6.0

REFERENCES 


\section{LIST OF FIGURES}

$\underline{\text { Figure }}$

2. 1-1

2.2-1

2. 2-2

2.2-3

$3.1-1$
Title

Tensile Strength

Stress to Rupture and Creep (Temperature) ' : .

Stress to Rupture and Creep (Time)

Stress vs. Strain (Temperature)

Thermal Expansion and Conductivity 


\subsection{BASIC DATA}

\subsection{GENERAL}

TZM Molybdenum alloy is superior in stress-rupture strength and recrystallization temperature to either pure molybdenum or the $0.5 \mathrm{Ti}$ - Molybdenum alloy. Fabricability and machinability are at least equal to Mo or Mo-0.5 Ti.

Values of $F_{T U}$ and $F_{T Y}$ are $95 \%$ confidence values.

\section{I.2 COMMERCIAL DESIGNATIONS}

Molybdenum - 0.5.titanium - 0.08 zirconium alloy.

Available Forms, Specifications, Suppliers

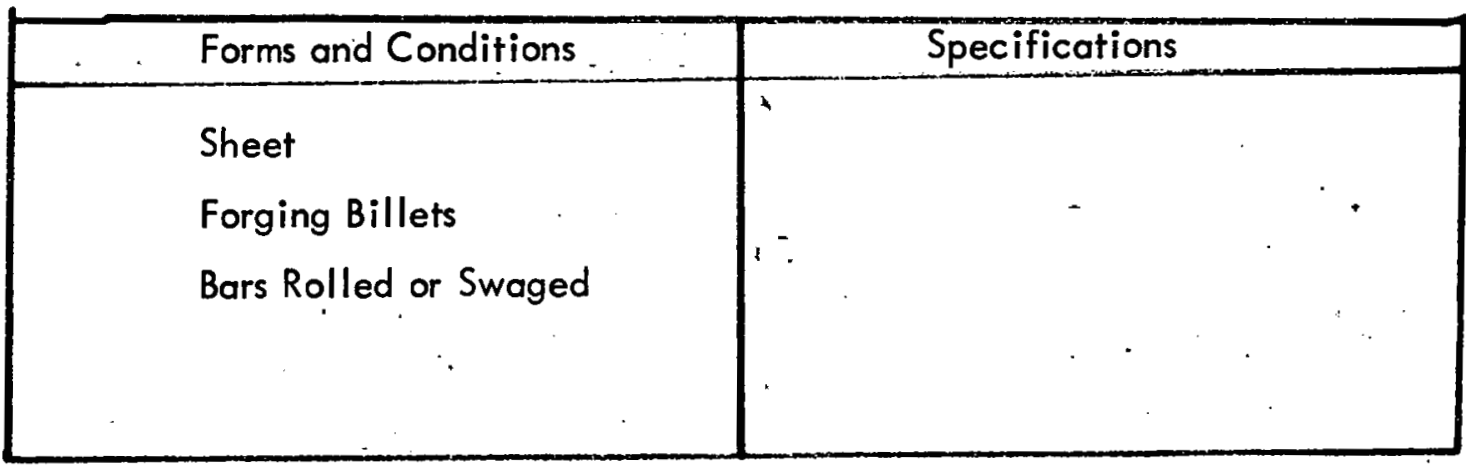

Suppliers

1.3 CHEMICAL COMPOSITION (\%)

$\begin{array}{lcccccccc}\text { ELEMENT } & \mathrm{C} & \mathrm{Ti} & \mathrm{Zr} & \mathrm{H} & \mathrm{Fe} & \mathrm{Ni} & \mathrm{N} & \mathrm{O} \\ \text { MAXIMUM } & 0.04 & 0.55 & 0.12 & .0005 & .010 & .002 & .002 & .0025 \\ \text { MINIMUM } & 0.01 & 0.04 & 0.06 & - & - & - & -\end{array}$


1.4 ROOM TEMPERATURE PROPERTIES

(Average Unless Otherwise Noted)

1.4.1 Mechanical Properties

$$
\text { Sheet, Annealed }
$$

Tensile Ultimate Strength

F TU:

PSI

$$
120,000 \text {. }
$$

Tensile Yield Strength

$$
\text { FYY } \frac{}{101,000}
$$

Comp. Yield Strength

${ }^{F_{C Y}}$

PSI

Shear Strength $\mathrm{F}_{\mathrm{SU}}$

PSI

Ultimate Bearing Strength

$F_{\text {BRU }}$

PSI

Elongation

e

$\%$

16.0

Reduction of Area

RA \%

55.0

Modulus of Elasticity

E $10^{6}$ PSI

(Static)

45.0

Modulus of Rigidity

G.

$10^{6} \mathrm{PSI}$

Comp. Modulus of Elasticity

${ }^{E} \mathrm{C}$

$10^{6} \mathrm{PSI}$

Plastic Bending Modulus

\subsubsection{Physical Properties}

Density

$\begin{array}{ll}\mathrm{LB} / \mathrm{IN}^{3} & 0.369\end{array}$

$\mathrm{g} / \mathrm{cc}$ 


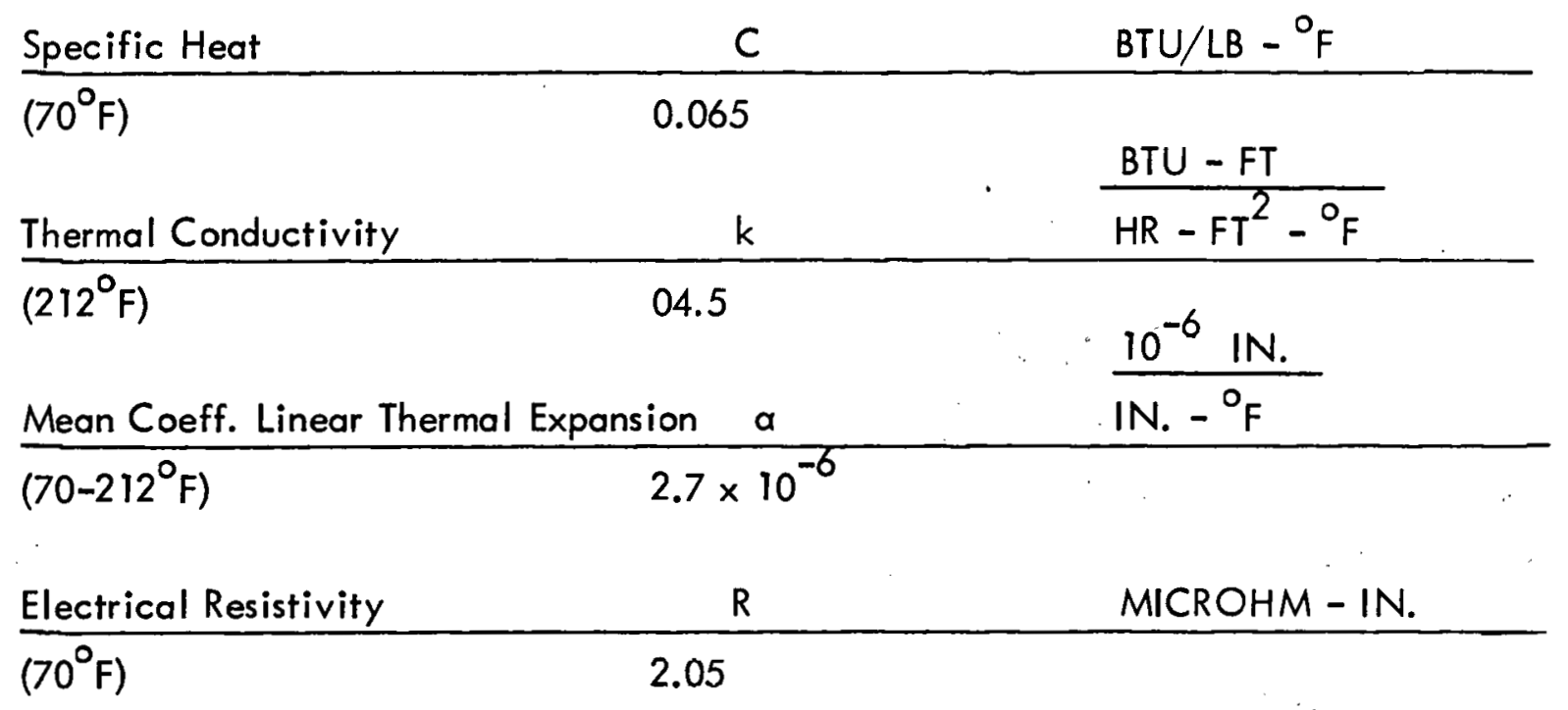

\subsubsection{Magnetic Properties}

Paramagnetic.

\subsubsection{Chemical Properties}

This alloy needs protection against oxidation at temperatures above $1000^{\circ} \mathrm{F}$, unless the exposure times are very short, or the atmosphere is neutral, reducing, or vacuum. Prolonged exposure to hydrogen above $1000^{\circ} \mathrm{F}$ can lead to internal hydriding of alloying elements $\mathrm{Zr}$ and Ti.

\subsubsection{Nuclear Properties}

\subsection{SPECIAL CONSIDERATIONS}

\section{Service Limits}

RT to $1000^{\circ} \mathrm{F}$ in oxidizing atmosphere without surface protection.

1.6 PROCESSING 
Recrystallization temperature depends on the extent of prior reduction.

Annealing and stress relief must be performed in a neutral or reducing atmosphere.

Stress Relief: $\quad 2100^{\circ}-2400^{\circ} \mathrm{F} ., 15 \mathrm{~min} .-1 \mathrm{hr}$.

Annealing: $\quad 2500^{\circ} \mathrm{F}-2550^{\circ} \mathrm{F}$., Hold $\mathrm{l} \mathrm{hr}$.

This alloy is not hardenable by heat treatment. It can be hardened by mechanical deformation at temperatures below the recrystallization temperature.

\subsection{JOINING}

This alloy can be fusion and resistance welded. A preheating to $400^{\circ}$ to $600^{\circ} \mathrm{F}$ is recommended. Through chemical cleaning on electroplating of surface to be joined is essential. Fusion welding should be done in an atmosphere free of nitrogen and oxygen.

\subsection{FORMING}

Sheet and wire up to 0.020 inch in stress relieved condition can be cold formed by conventional methods. Materials thicker than 0.020 in. should be heated at $200^{\circ}$ to $1000^{\circ} \mathrm{F}$, depending on section thickness. Preheating of tool is recommended.

Forging, starting temperatures is $2350^{\circ} \mathrm{F}$ maximum, and finishing temperature is $1900^{\circ} \mathrm{F}$ minimum. A GUIDE TO FABRICATION TEMPERATURE FOR TZM ALLOY

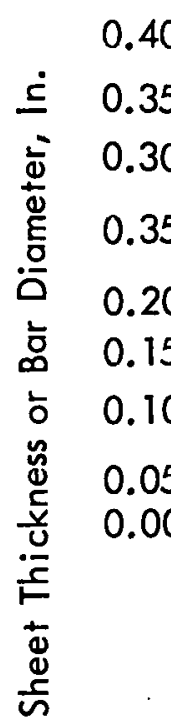

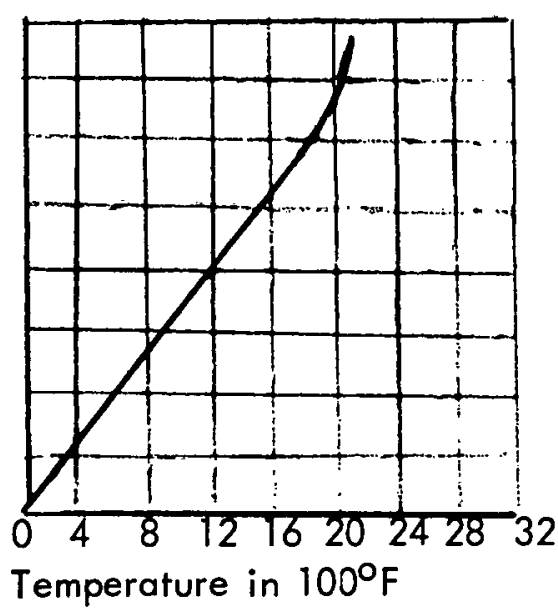




\subsection{MACHINABILITY}

The use of high-speed steel and carbide tools is recommended for this alloy.

Shearing and punching can be performed at $200^{\circ}$ to $1000^{\circ} \mathrm{F}$. 


\subsection{MECHANICAL PROPERTIES}

\subsection{SHORT TERM}

\subsubsection{Static}

Figure 2.1-1

Tensile Strength

\subsection{LONG TERM}

Figure 2.2-1

Stress to Rupture and Creep (Temperature)

Figure 2.2-2

Stress to Rupture and Creep (Time)

Figure 2.2-3

Stress vs. Strain (Temperature) 


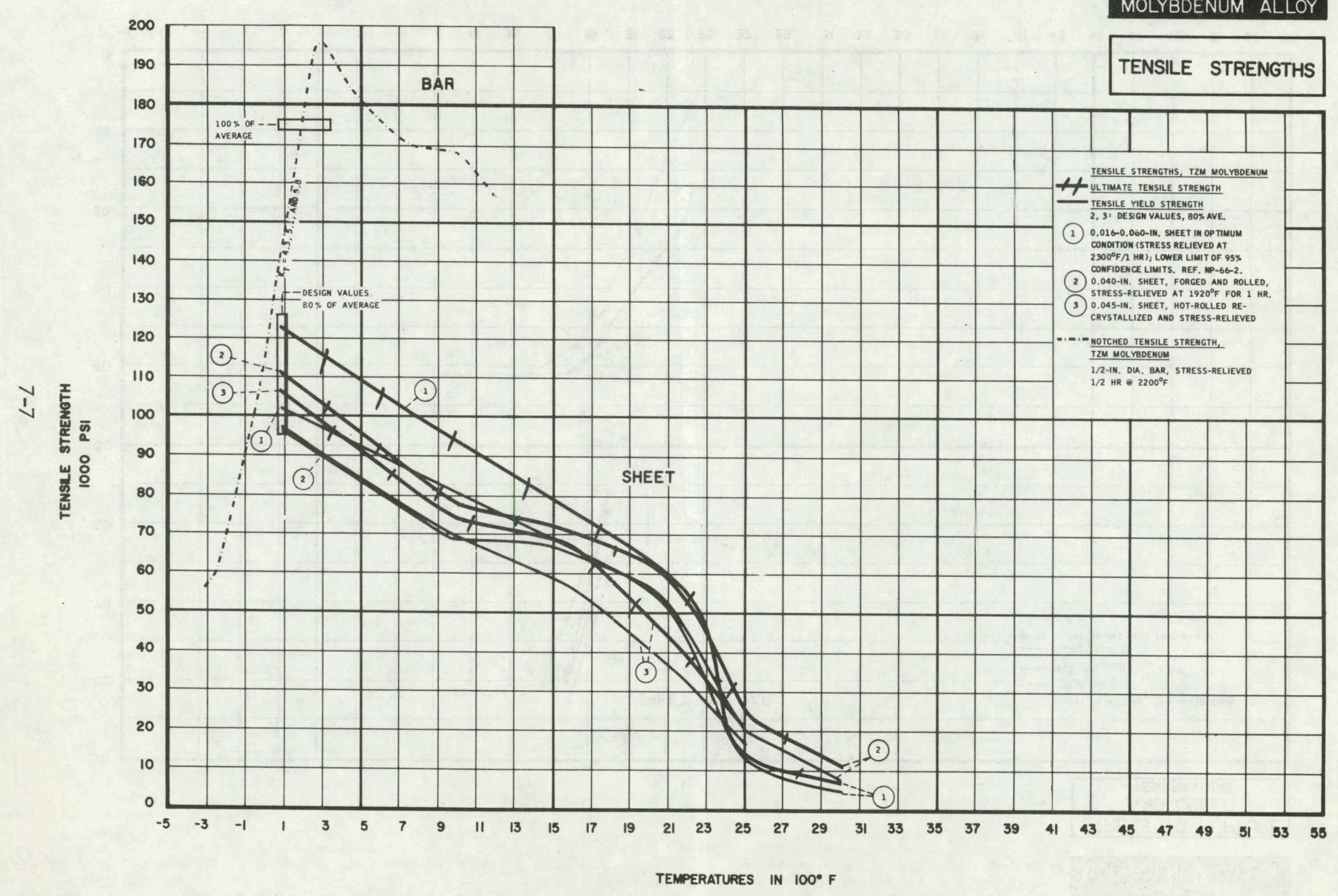

Figure 2.1-1 Tensile Strength 


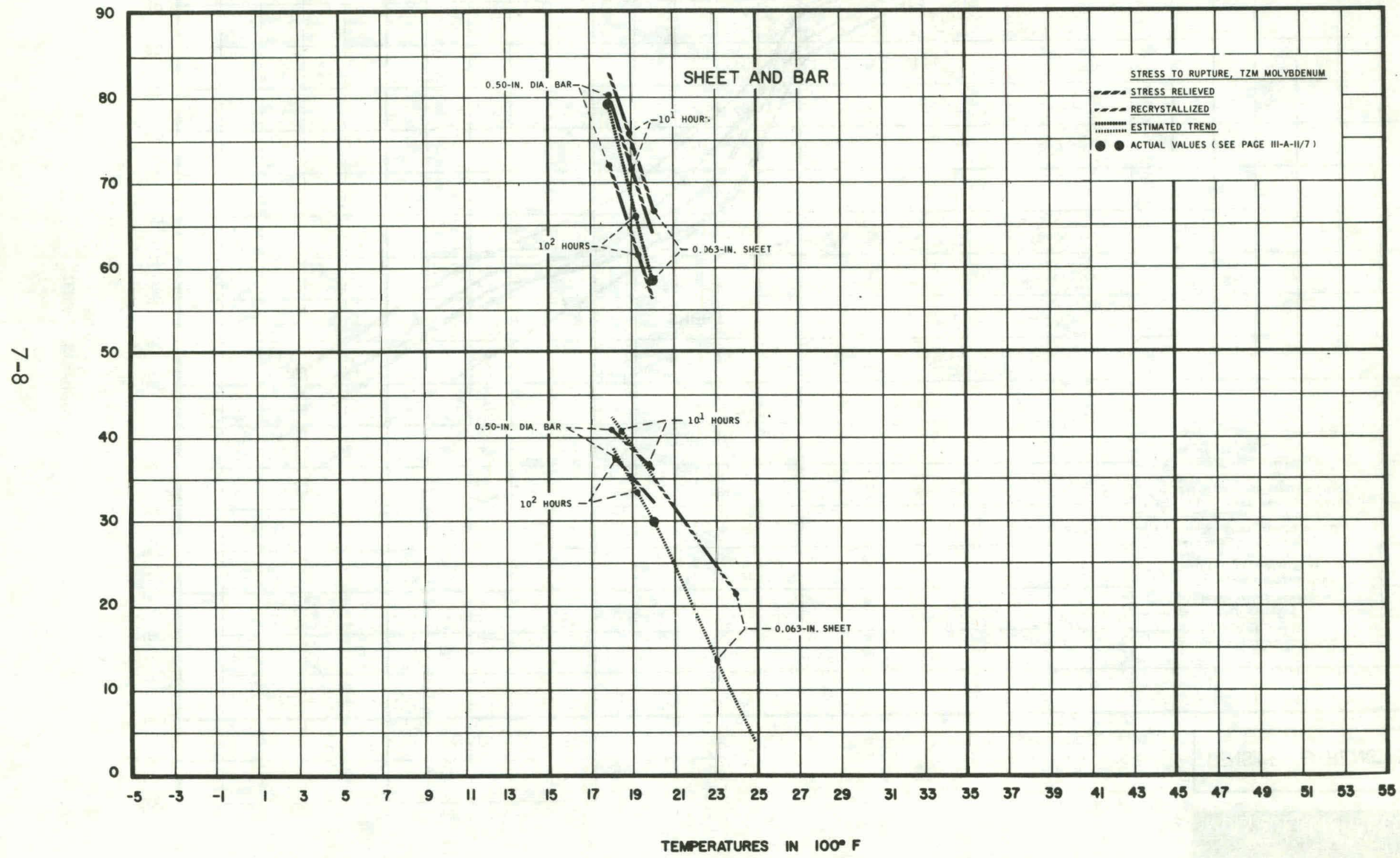

Figure 2.2-1 Stress to Rupture and Creep (Temperałure) 


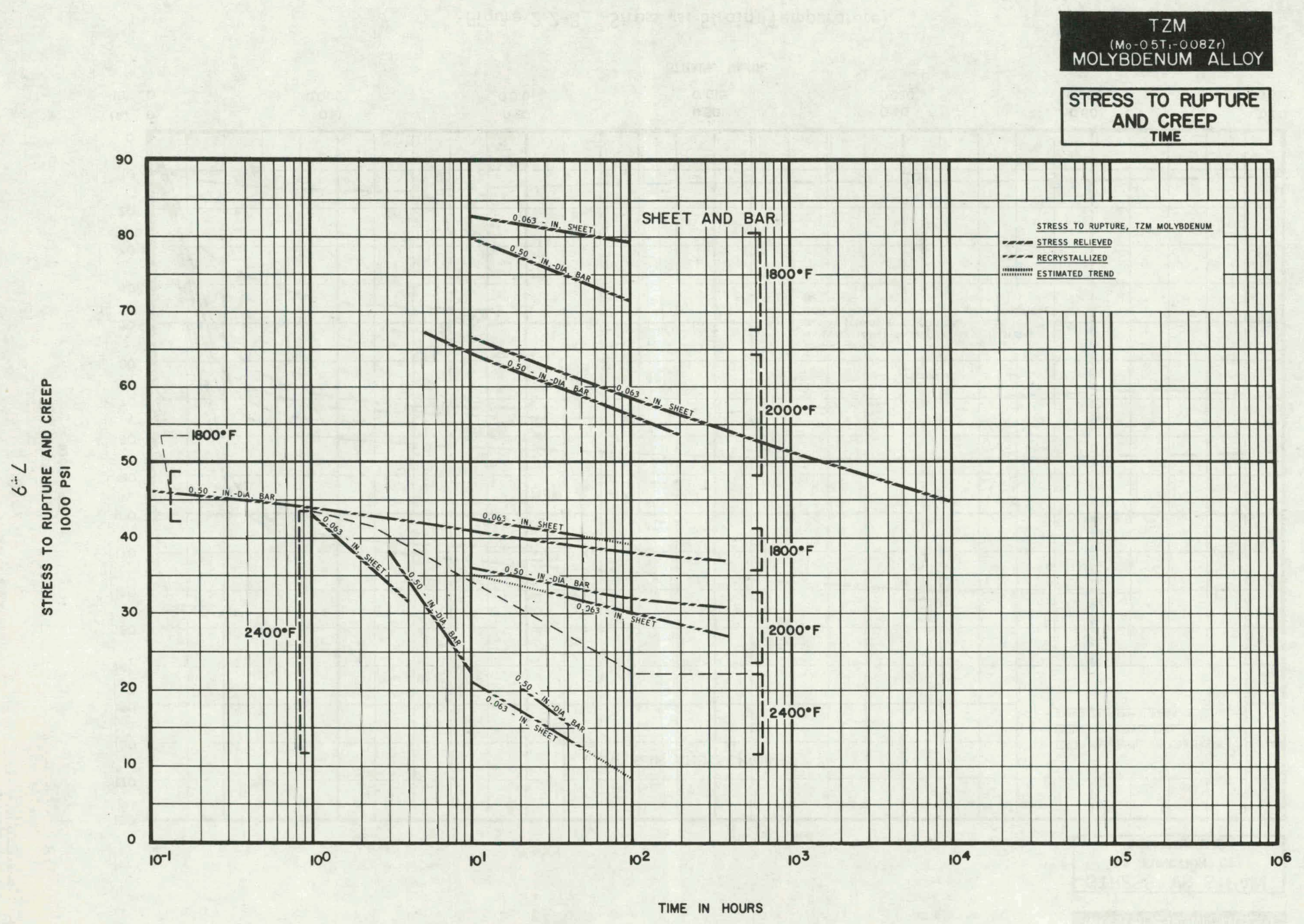

Figure 2.2-2 Stress to Rupture and Creep (Time) 
TEMPERATURE

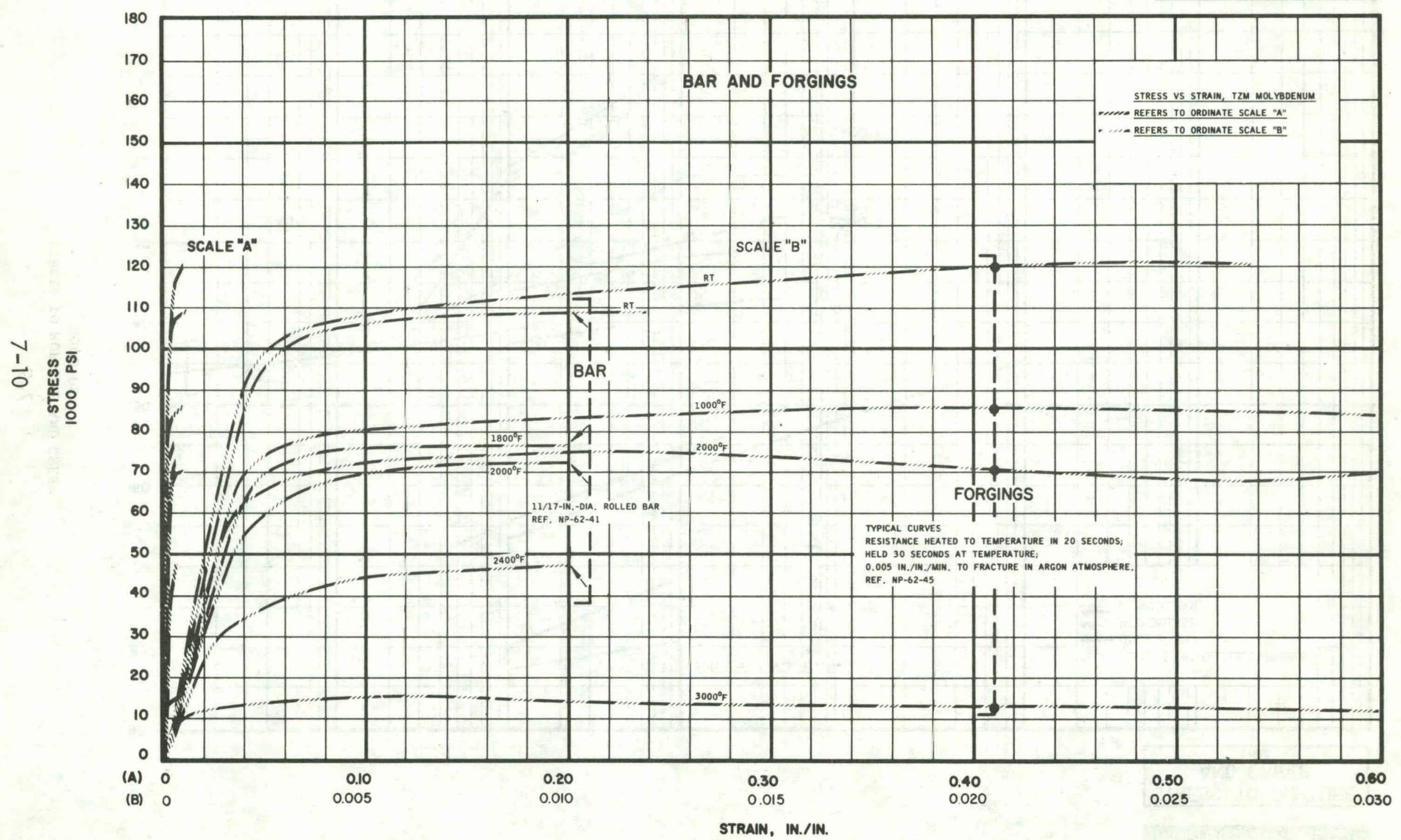

Figure 2.2-3 Stress vs. Strain (Temperature) 


\subsection{PHYSICAL PROPERTIES}

\section{I THERMAL PROPERTIES}

Figure $3.1-1$

Thermal Expansion and Conductivity

$7-11$ 
THERMAL EXPANSION VALUES ARE FROM

THERMAL EXPANSION

SPECIFIC HEAT

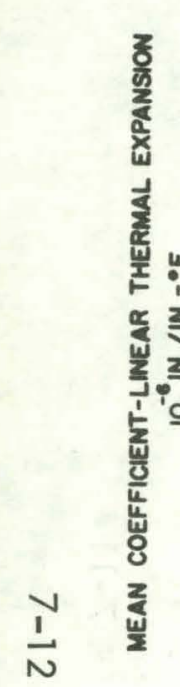

ROOM TEMPERATURE TO INDICATED TEMP

HEAT

2
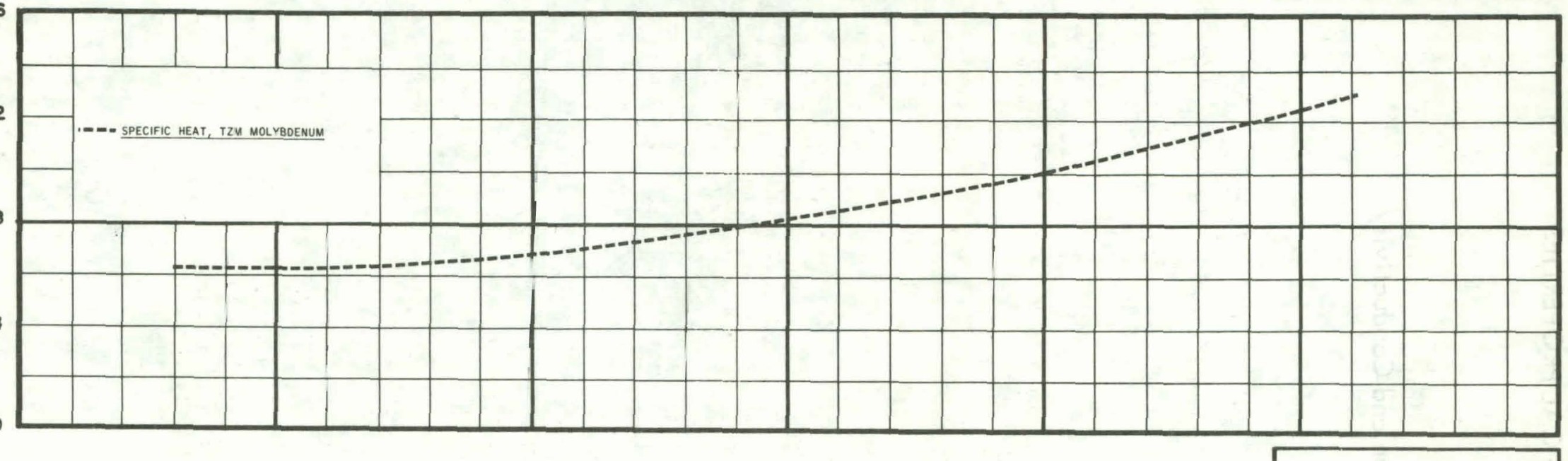

THERMAL CONDUCTIVITY

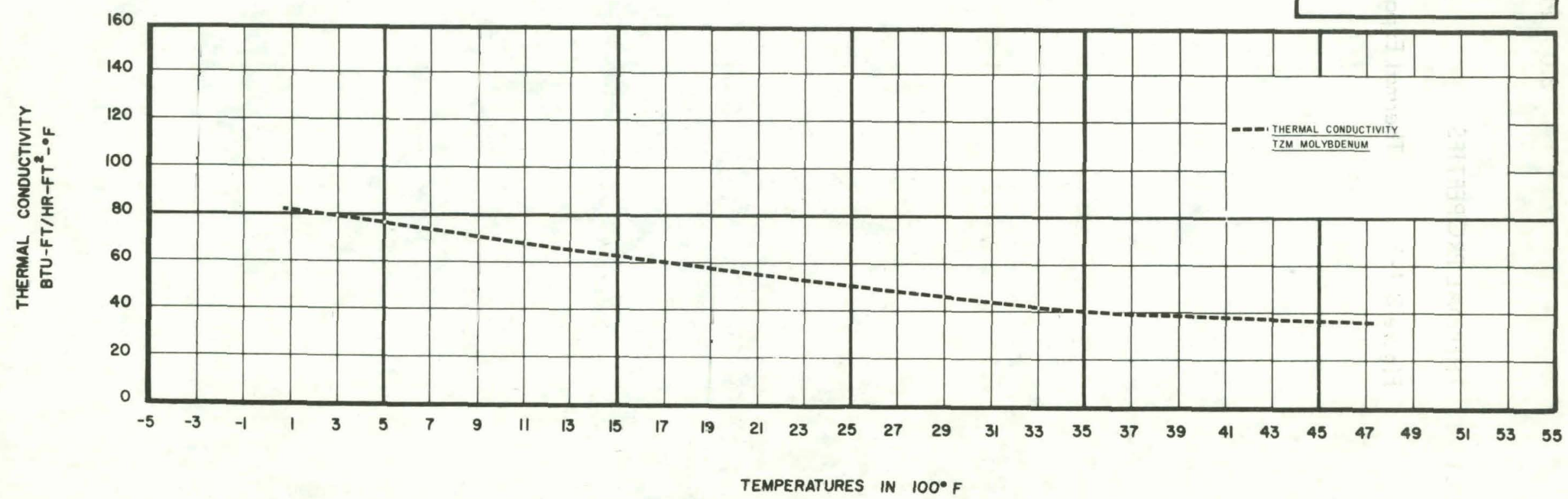

Figure 3.1-1 Thermal Expansion and Conductivity 
4.0 CHEMICAL PROPERTIES

$7-13$ 
5.0 NUCLEAR PROPERTIES

$7-14$ 


\subsection{REFERENCES}

6.1 Mechanical Properties of Tungsten and TZM Sheet Produced in the Refractory Metal Sheet Rolling Program, Part II, Contract N600 (19)-59530, Southern Research Institute, 17 Jan. 1966.

6.2 The Engineering Properties of Molybdenum and Molybdenum Alloys, DMIC Report, Battelle, September, 1963.

6.3 Mechanical Properties of Various Metals, Unpublished WANL. Test Data.

6.4 Aerospace Structural Metals Handbook, Syracuse University Imstitute.

6.5 TZM Development Data, Climax Molybdenum Company, January, 1962.

6.6 Determination of the Tensile Properties up to $4500^{\circ} \mathrm{F}$ and the Impact Ductile Brittle Transite Temperature of Unalloyed Molybdenum Forgings - Southern Research Institute Report 5376 - 1364 - XII, June, 1962. 
SECTION 8.0

TUNGSTEN 


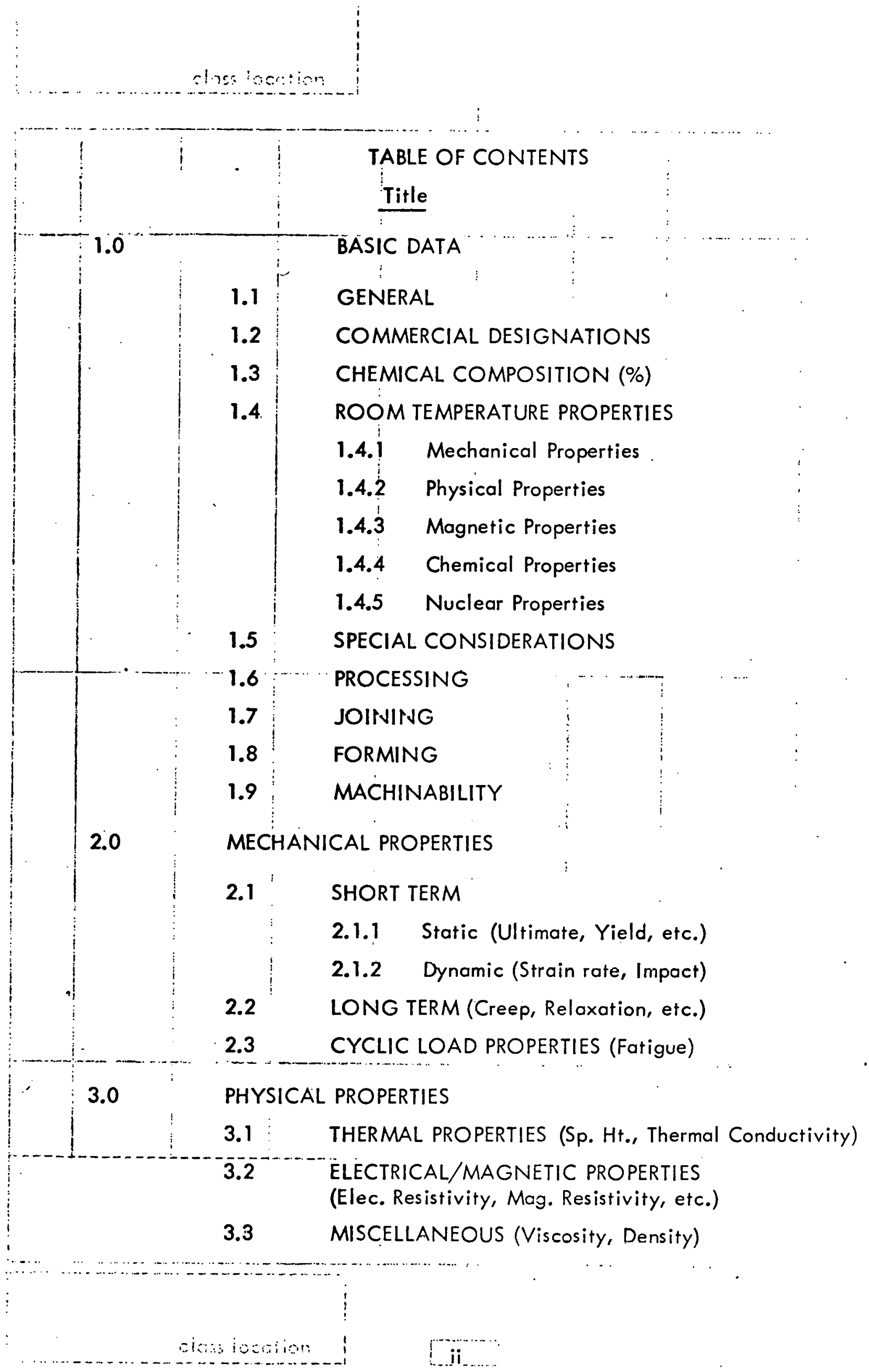


4.0 CHEMICAL PROPERTIES

5.0 NUCLEAR PROPERTIES

6.0 REFERENCES 


\section{LIST OF FIGURES}

\begin{tabular}{ll}
$\frac{\text { Figure }}{2.1-1}$ & \multicolumn{1}{c}{ Title } \\
$2.1-2$ & Tensile Strengths \\
$2.1-3$ & Tensile Strengths \\
$2.1-4$ & Reduction of Area \\
$2.1-5$ & Modulus of Elasticity \\
$3.1-1$ & Thermal Expansion and Conductivity \\
$3.2-1$ & Electrical Resistivity
\end{tabular}




\subsection{BASIC DATA}

\section{I GENERAL}

Tungsten is a refractory metal with the highest melting temperature of all of the metals. It is, however, extremely brittle at room temperature, with a ductile-brittle transition temperature of $350^{\circ} \mathrm{F}$ in the wrought condition and $600^{\circ} \mathrm{F}$ in the annealed condition. It has body-centered cubic structure, although other forms may exist.

Typical uses are as an alloying element, for electrical and electronic applications, missile and space applications, weights and counter balances in static or dynamic balancing, highspeed rotating inertia members, radiation shielding, and vibration damping.

\subsection{COMMERCIAL DESIGNATIONS}

Commercially pure tungsten, or commercially pure nonsag tungsten.

Available Forms, Specifications, Suppliers

\begin{tabular}{|l|l|}
\hline Forms \& Conditions & - Specifications \\
\hline Powder & MIL-T-13827 \\
\hline Wire & MIL-T-14507 \\
& ASTM-F-288, AMS-7726, \\
\hline Rod & MIL-T-14507 \\
\hline Billet, Bar, Rod, and Preforms & ASTM-B-410 \\
\hline Plate, Sheet, Strip, and Foil & AMS-7898 \\
& AMS-7899 \\
\hline $\begin{array}{l}\text { High Density, Sintered or Hot- } \\
\text { Pressed }\end{array}$ & MIL-T-2 1014 \\
\hline
\end{tabular}




\begin{tabular}{|l|c|c|c|}
\hline ELEMENT & $W$ & $\mathrm{O}_{2}$ & $\mathrm{~N}_{2}$ \\
\hline MAXIMUM & $99-99.99 \%$ Pure & .005 & .010 \\
\hline MINIMUM & - & - & - \\
\hline
\end{tabular}

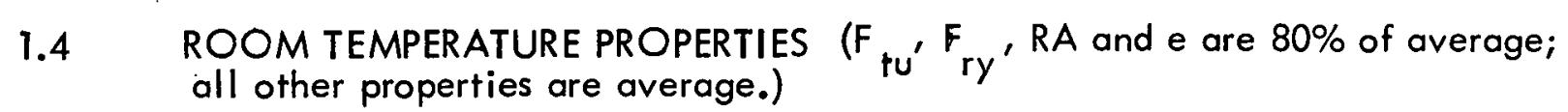

1.4.1 Mechanical Properties

Tensile Ultimate Strength

Comm. Pure Sheet, 0.062-in. SR

F TU

166,000

Tensile Yield Strength

F TY

PSI

Comp. Yield Strength

$\mathrm{F}_{\mathrm{CY}}$

PSI

200,000

Ultimate Shear Strength

$\mathrm{F}_{\mathrm{SU}}$

PSI

Shear Yield Strength

$F_{S Y}$

PSI

Ultimate Bearing Strength

$F_{B R U}$

PSI

$\mathrm{e} / \mathrm{D}=1.5$

$e / D=2.0$

Bearing Yield Strength

$F_{\text {BRY }}$

PSI

$\mathrm{e} / \mathrm{D}=1.5$

$e / D=2.0$ 
e

Longitudinal

Transverse
2.4

0.1

$\%$

$<1$

Sheet, SR

RA

Reduction of Area

Modulus of Elasticity

E

$10^{6} \mathrm{PSI}$

Sheet, 0.062-In.

RT

$1830^{\circ} \mathrm{F}$

58.5

93

Comp. Modulus of Elasticity

${ }^{F}$ C

$10^{6} \mathrm{PSI}$

Poisson's Ratio

Hardness

Sheet, SR.

45

Recrystallized

36

\subsubsection{Physical Properties}

Plate. Sheet

Density

$\rho$

$\begin{array}{lcc}\mathrm{LB} / \mathrm{IN}^{3} & 0.697 & 0.689 \\ \mathrm{~g} / \mathrm{cc} & 19.3 & 19.07\end{array}$

Specific Heat

C

$B T U / L B-{ }^{\circ} \mathrm{F}$

0.033

Thermal Conductivity

k

$\frac{B T U \div F T}{H R-F T^{2}-{ }^{\circ} F}$

RT

$932^{\circ} \mathrm{F}$ 


\begin{tabular}{lll} 
Mean Coeff. Lin. Thermal Expan. & $\alpha$ & $\frac{10^{-6}-\mathrm{IN}}{1 \mathrm{~N} .-{ }^{\circ} \mathrm{F}}$ \\
\hline RT to $1000^{\circ} \mathrm{F}$ & 2.45 & \\
RT to $3600^{\circ} \mathrm{F}$ & 3.0 & \\
Electrical Resistivity & $\mathrm{R}$ & MICROHM-IN.
\end{tabular}

\subsubsection{Magnetic Properties}

\section{Paramagnetic}

\subsubsection{Chemical Properties}

Resists attack by most dilute acids and alkalies at $R T$, slightly attacked by concentrated acids. Rapidly attacked by hot concentrated acids and alkalies.

Regins to oxide in air at red heat.

Can be used in vacuum and hydrogen to temperatures well above $2000^{\circ} \mathrm{F}$.

\subsubsection{Nuclear Properties}

Few data are available on the effects of radiation on tungsten after exposures of $1 \times 10^{19}$ NVT (slow) and $5 \times 10^{19} \mathrm{NVT}$ (fast) neutrons. No significant change in the hardness was observed, but the electrical resistivity decreased nearly $40 \%$. The density decreased $0.1-0.15 \%$. After similar exposure a decrease of approximately $22 \%$ was observed in the tenslle strength of tungsten wire. However, the samples were brittle and difficult to align in the tensile grips and the decrease may not be entirely related to the radiation damage.

\subsection{SPECIAL CONSIDERATIONS}

Service Limits 
In air to $300^{\circ} \mathrm{F}$

In vacuum to $3000^{\circ} \mathrm{F}$

In hydrogen to $4500^{\circ} \mathrm{F}$

Do not use in oxidizing atmospheres

\subsection{PROCESSING}

\section{Surface Treatment}

After forming, either "cold" or "hot", in reducing atmospheres, degrease by conventional methods.

After heating in oxidizing atmosphere, remove oxide coating by momentary immersion in molten caustic soda, followed by drainage, cooling, and rinsing in hot running water.

\section{Thermal Treatment}

Sinter in vacuum or hydrogen at $3600^{\circ}$ to $5400^{\circ} \mathrm{F}$.

Stress-relief anneal from $1100^{\circ}$ to $1850^{\circ} \mathrm{F}$, depending on time, purity, and degree of cold work.

Heating above $750^{\circ} \mathrm{F}$ should be done in argon, hydrogen, or dissociated ammonia, except for very thin sheet or wire, protective atmosphere should be used above $500^{\circ} \mathrm{F}$.

\subsection{JOINING}

Welding not recommended because of brittleness. Furnace brazing satisfactory below $3000^{\circ} \mathrm{F}$.

\subsection{FORMING}

Initial forging, rolling, or swaging of sintered compacts at $2900^{\circ}$ to $3300^{\circ} \mathrm{F}$.

Working temperature ranges from $750^{\circ} \mathrm{F}$ in the "cold" ranige to $3000^{\circ} \mathrm{F}$ in the "hot" range.

Work must be done quickly to overcome the high thermal conductivity and low specific heat. 
Use minimum bend radius of $2 \mathrm{~T}$.

\subsection{MACHINABILITY}

High density forms, whether produced by severe sintering or by mechanical working, are the most difficult of all metals to machine, although they are machinable with proper techniques and tools, fairly good speeds and feeds are recommended; generally, no lubricant is required.

Below $90 \%$ of theoretical density, ease of chip removal is inversely related to density. In the extreme of commercial availability, approximately $70 \%$ of theoretical density, chips can be removed as readily as from ferritic cast iron. 


\subsection{MECHANICAL PROPERTIES \\ (As Function of Temperature)}

\subsection{SHORT TERM}

\subsubsection{Static}

Figure 2. 1-1

Figure 2. 1-2

Figure 2. 1-3

Figure 2. 1-4

Figure 2.1-5
Tensile Strengths

Tensile Strengths

Tensile Strengths

Reduction of Area

Modulus of Elasticity 


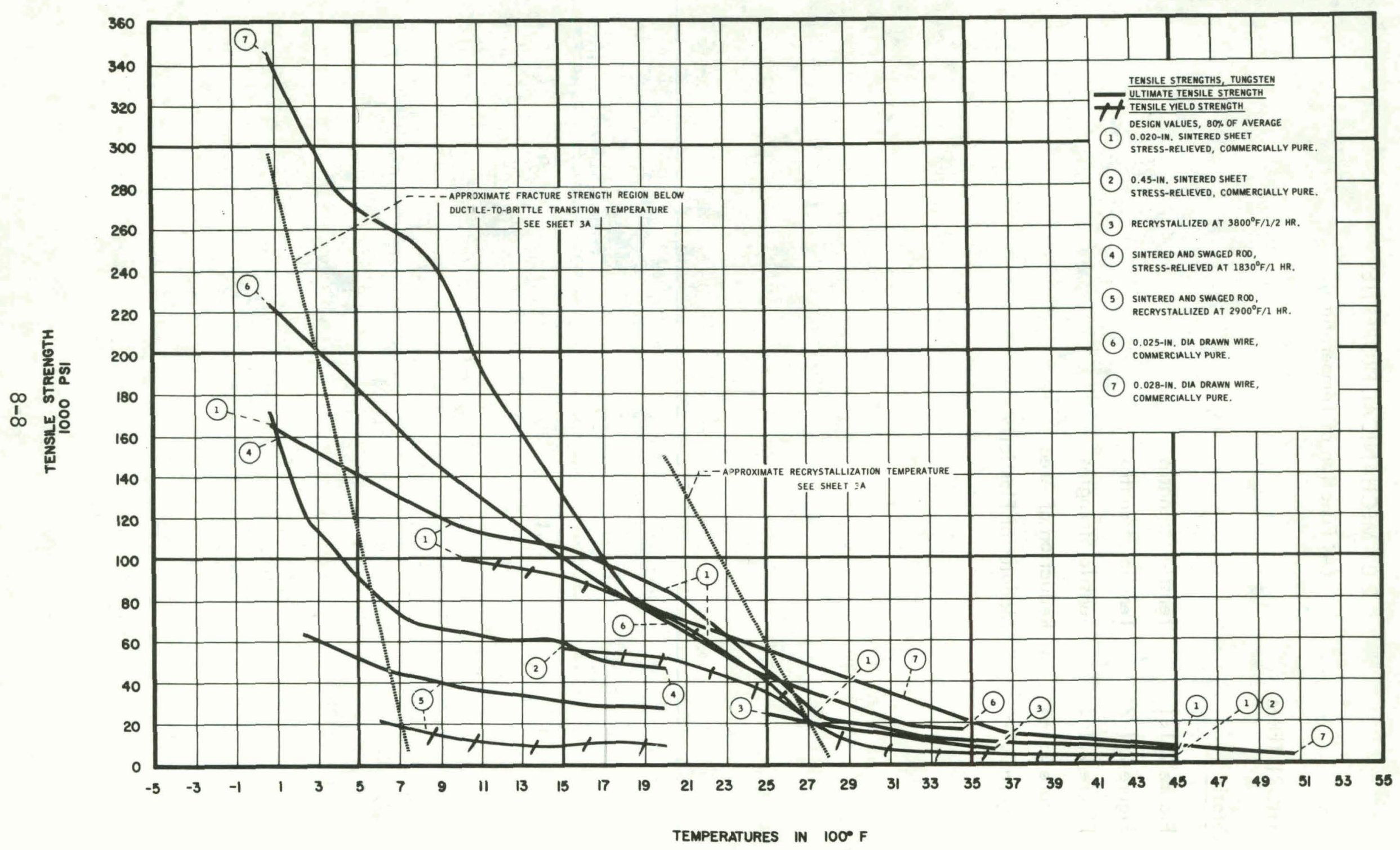

Figure 2.1-1 Tensile Strengths 


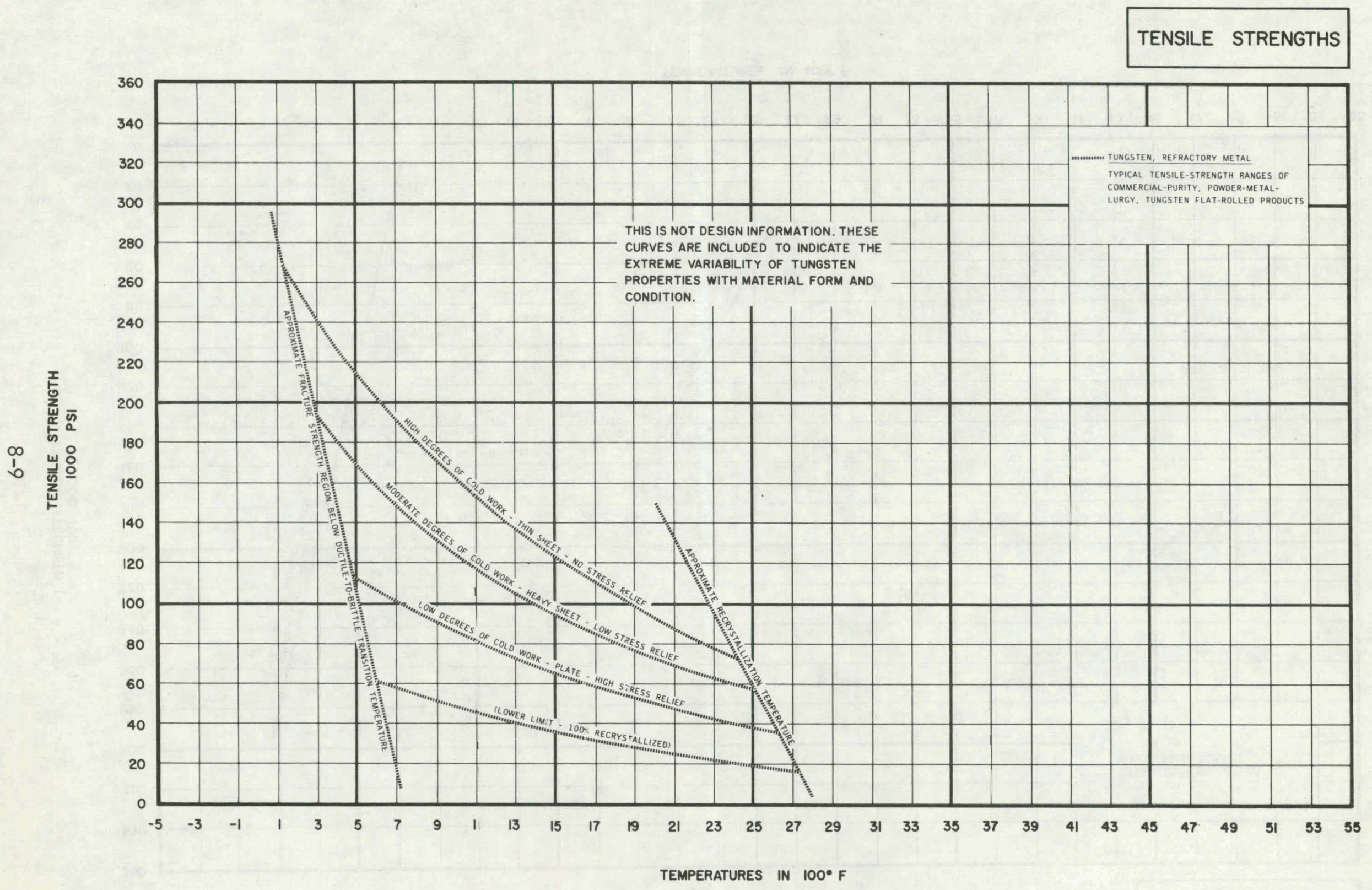

Figure 2.1-2 Tensile Strengths 
TENSILE STRENGTHS

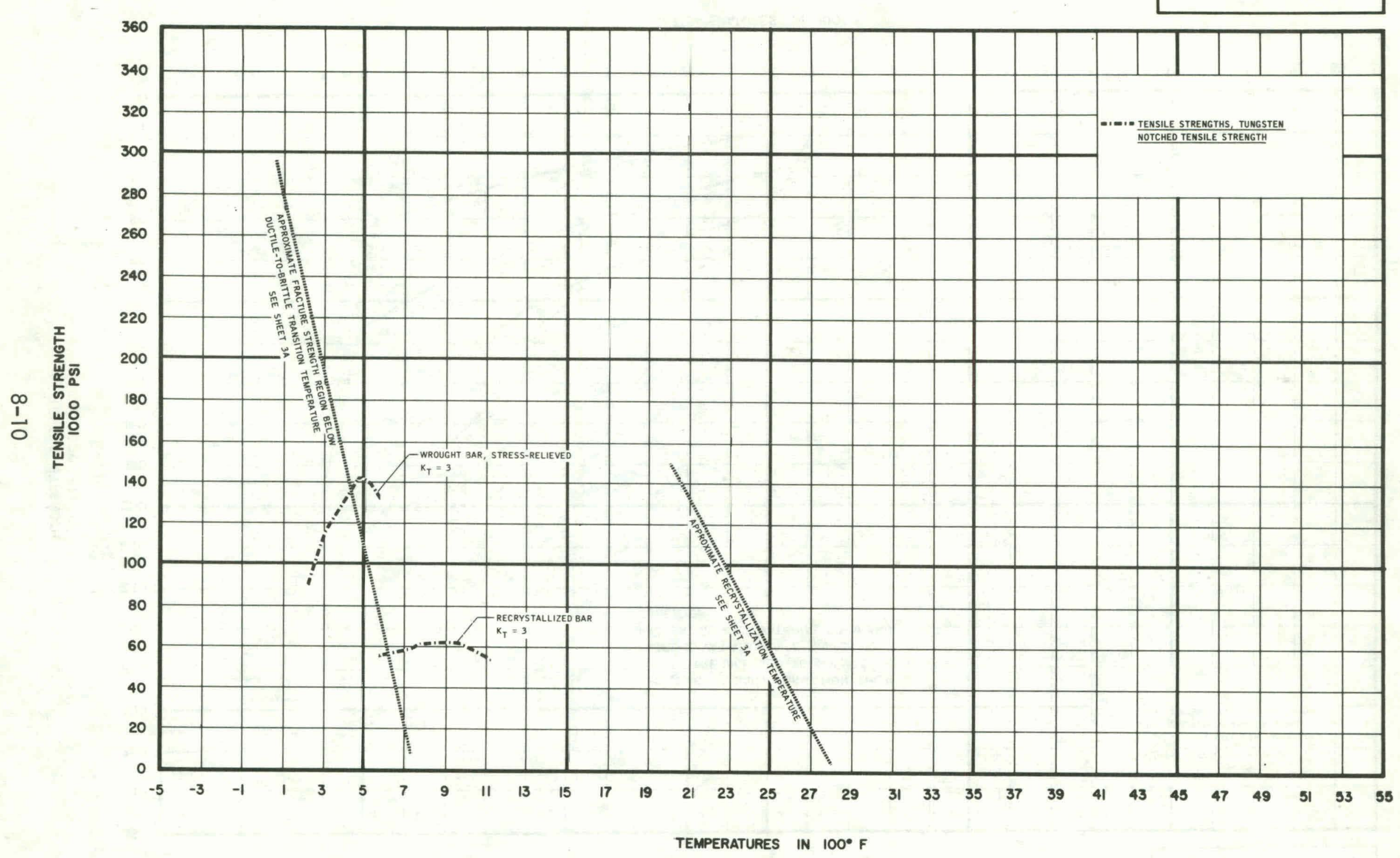

Figure 2. "-3 Tensile Strengths 


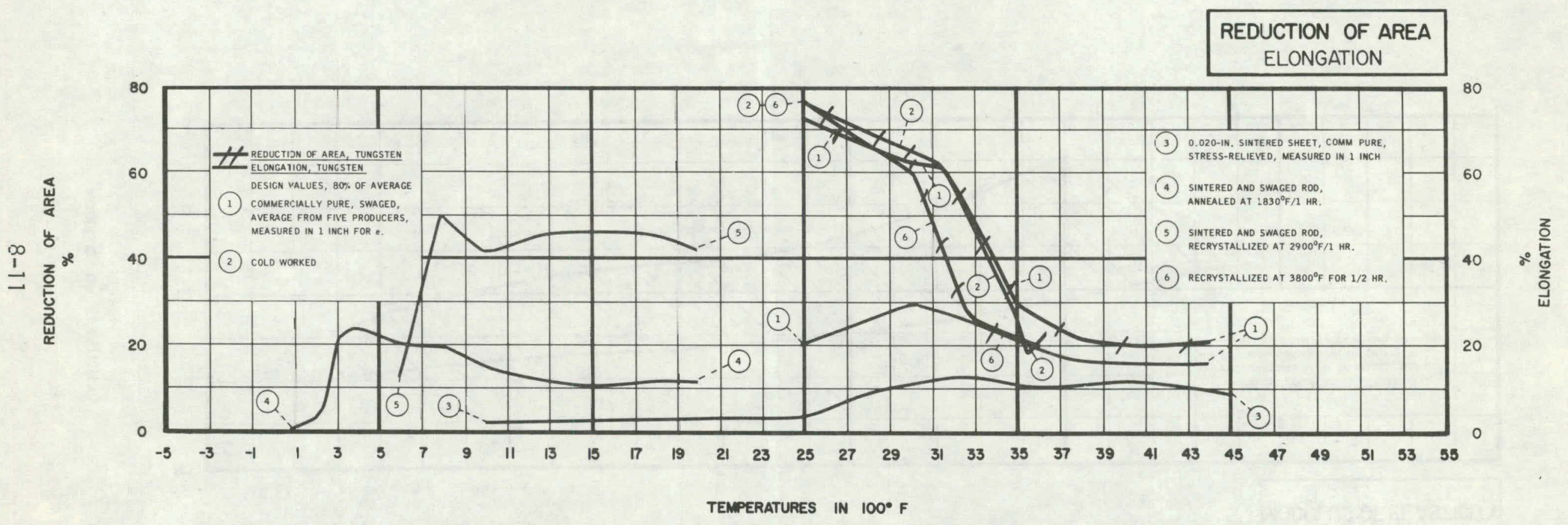

Figure 2.1-4 Reduction of Area 


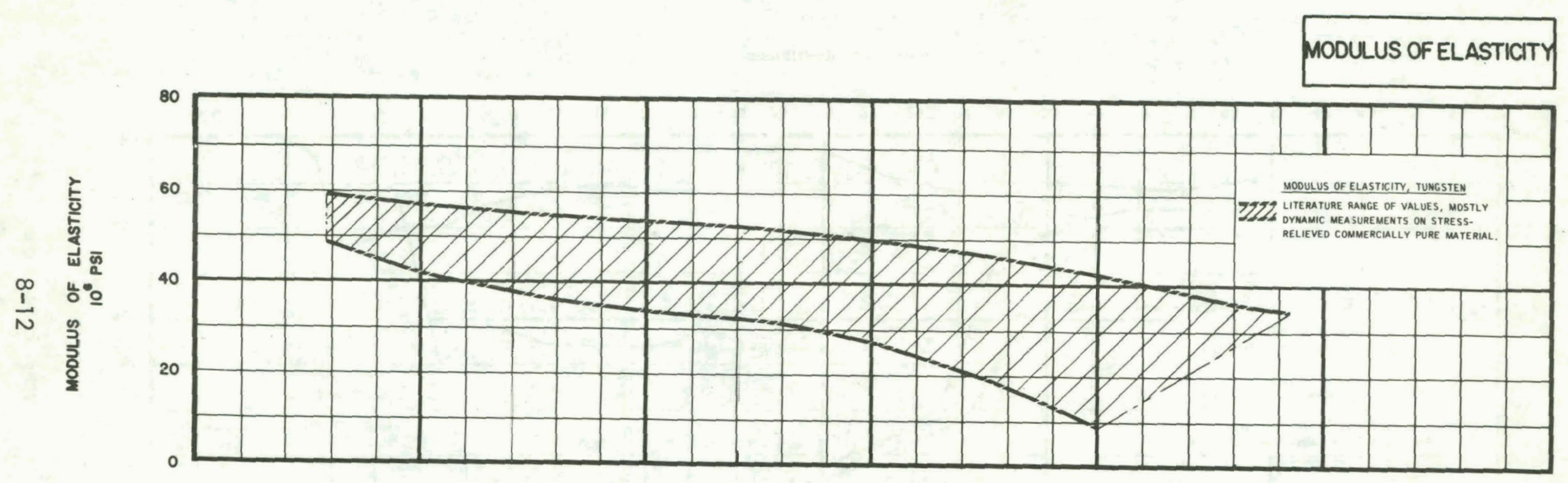

Figure 2.1-5 Modulus of Elasticity 
3.0 PHYSICAL PROPERTIES

\subsection{THERMAL PROPERTIES}

Figure 3.1-1 Thermal Expansion and Conductivity 


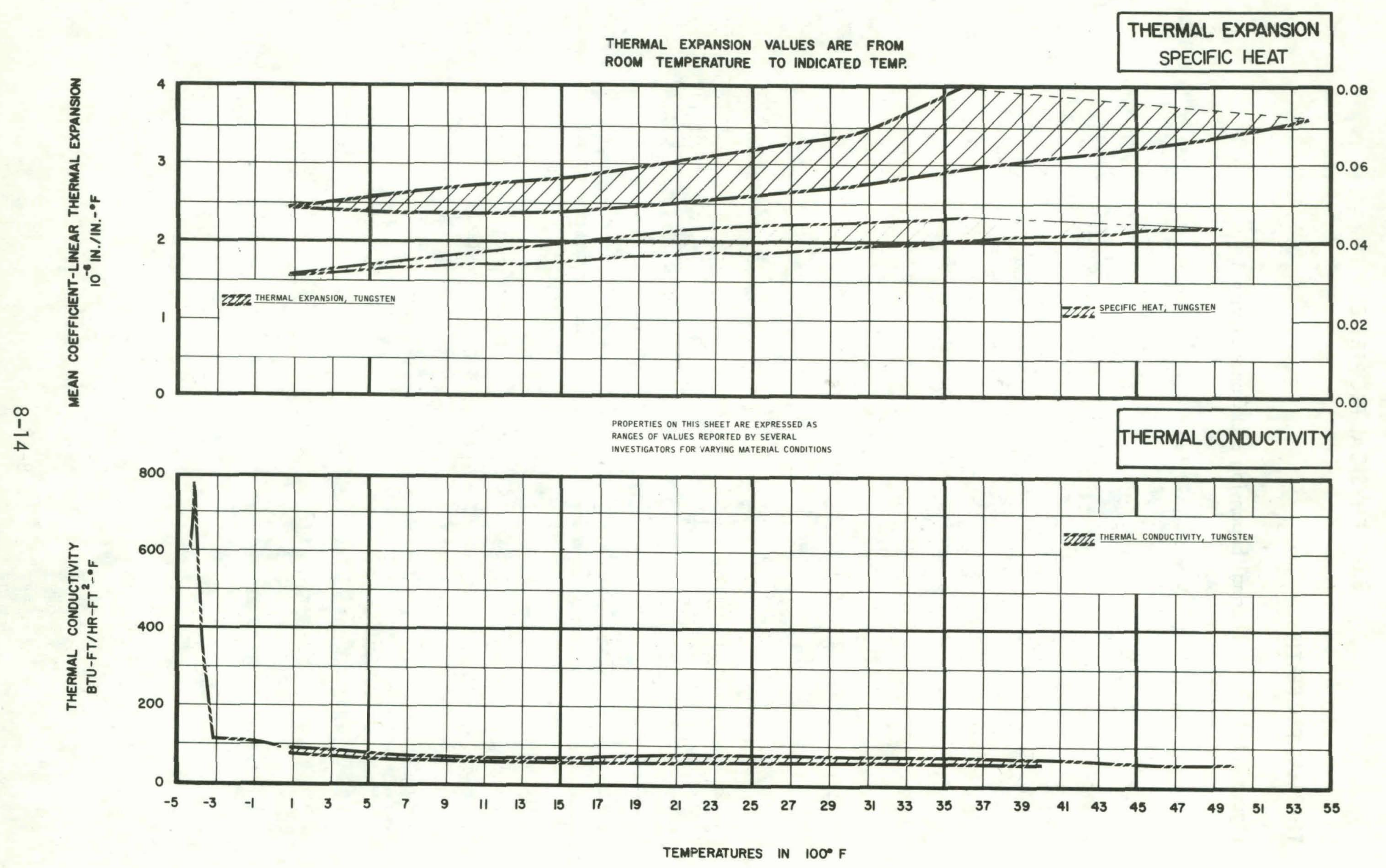

Figure 3.1-1 Thermal Expansion and Conductivity 
3.2 ELECTRICAL/MAGNETIC PROPERTIES

Figure 3.2-1 Electrical Resistivity 


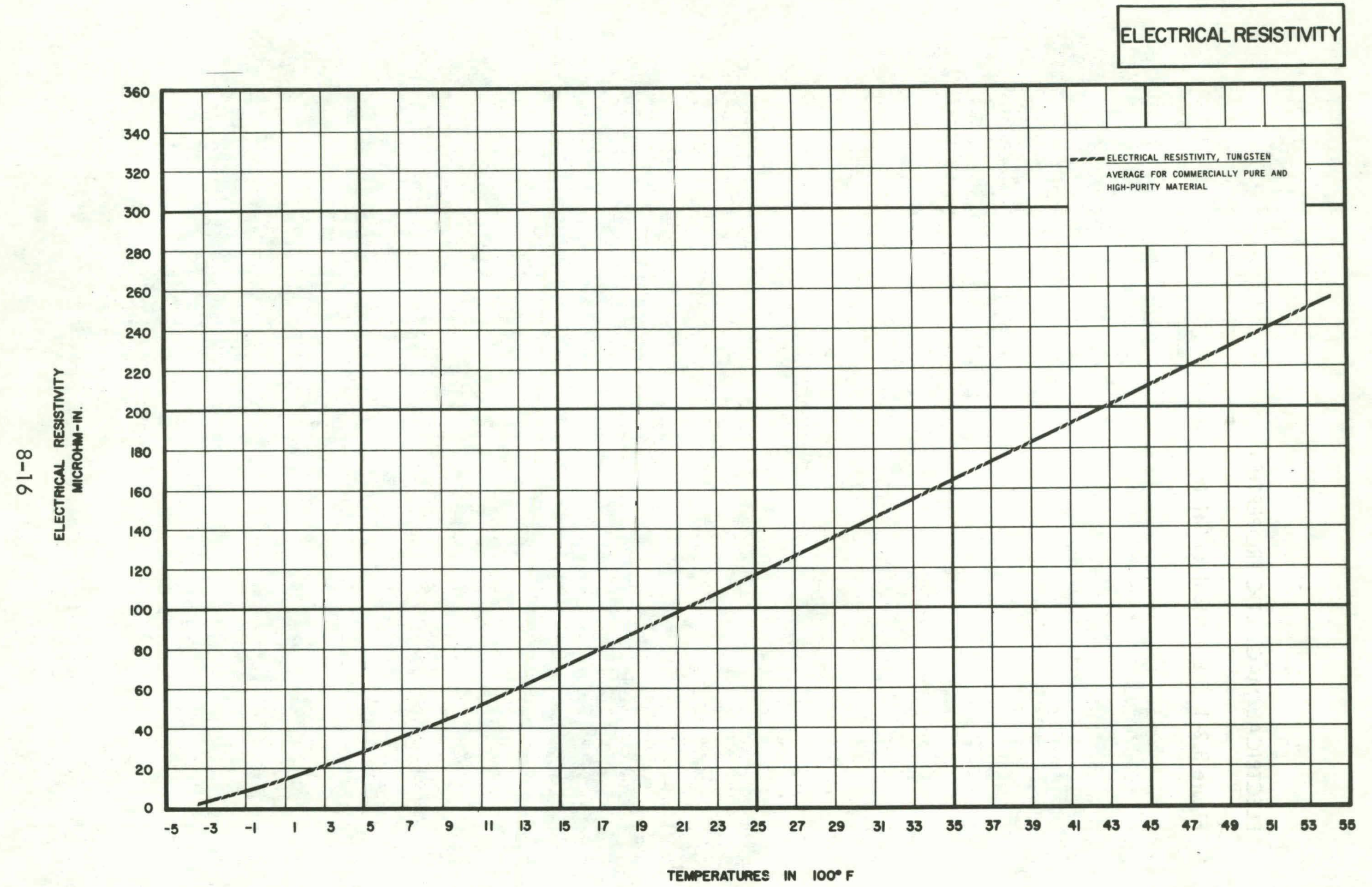

Figure 3.2-1 Electrical Resistivity 
4.0 CHEMICAL PROPERTIES 
5.0 NUCLEAR PROPERTIES

$8-18$ 


\subsection{REFERENCES}

6.1 F. F. Schmidt and H. R. Ogden, "Engineering Properties of Tungsten and Tungsten Alloys," DMIC Report 191, Battelle, September, 1963.

6.2 T. W. Pugh, "Tensile and Creep Properties of Tungsten at Elevated Temperatures," ASTM Proceedings, Vol. 57, 1957.

6.3 "Typical Properties of Tungsten, Tantalum, Molybdenum, and Columbium," Fansteel Metallurgical Co., 1960.

6.4 A. S. Ingram, et al, "Notch Sensitivity of Refractory Metals, "Battelle Report, WADD-TR -60-258, September, 1960.

6.5 P. E. Armstrong and H. C. Brown, "Dynamic Young's Modulus Measurements above $1000^{\circ} \mathrm{C}$ on Some Pure Polycrystalline Metals and Commercial Graphites," Trans. of Metallurgical Society of AIME, Vol. 230, August, 1964.

6.6 Aerospace Structural Metals Handbook, Syracuse University Institute.

6.7 A. Goldsmith, et al, Armour Research Foundation, Handbook of Thermophysical Properties of Solid Materials, Volume 1-5, Rev. Ed. WADC-TR-58-476, The Maxmillan Co., N. Y., 1961.

6.8 Sylvania Tungsten, Chemical and Metallurgical Division, Sylvania, Towanda, N. Y. 1962.

6.9 W. D. Wood and H. W. Deem, Thermal Properties of High Temperature Materials Battelle Report RSIC-202 (for U. S. Army Missile Command, Redstone Arsenal), June, 1964. 
SECTION 9.0

\section{OXYGEN-FREE COPPER}




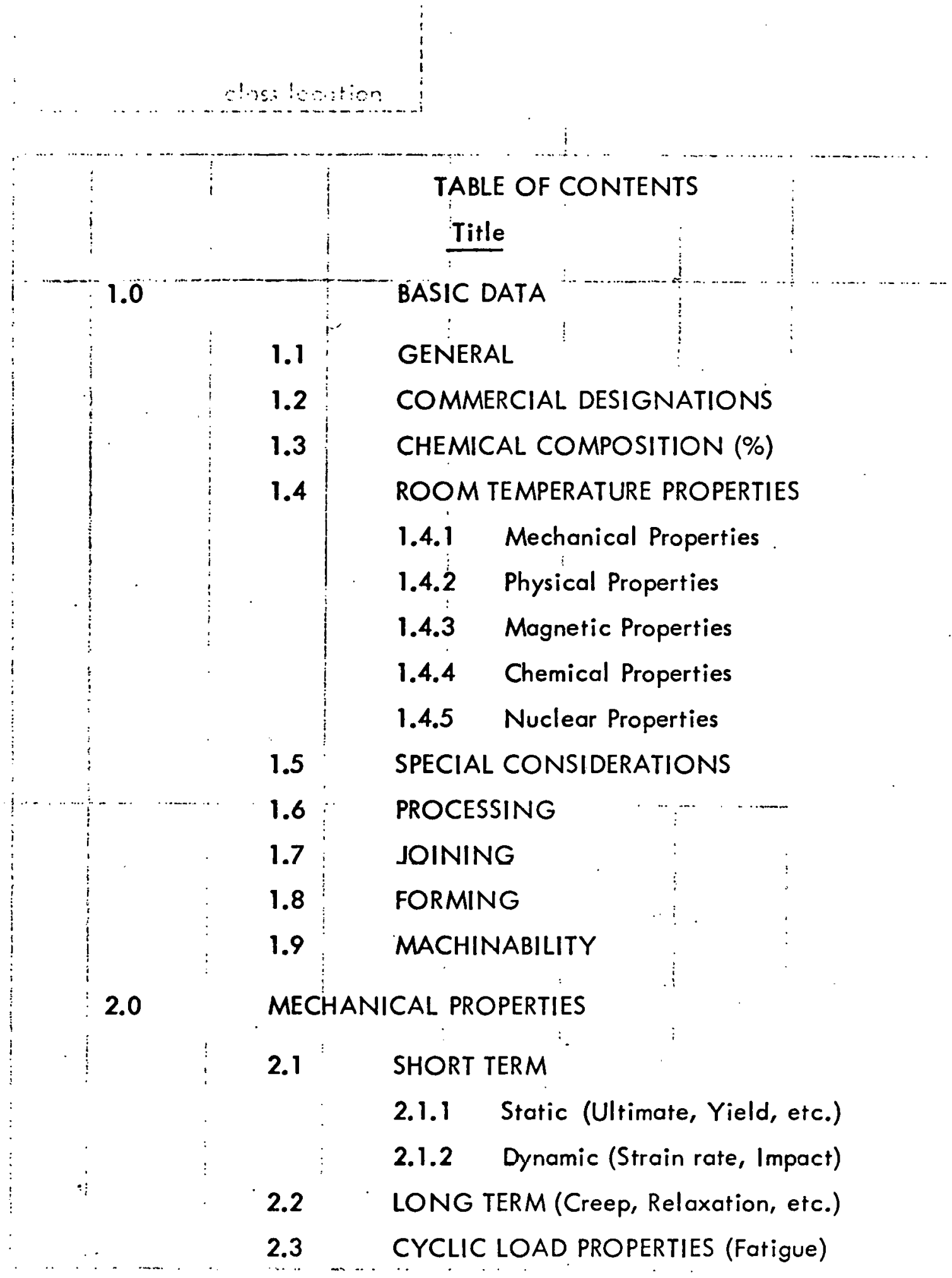

3.0 PHYSICAL PROPERTIES

3.1 THERMAL PROPERTIES (Sp. Ht., Thermal Conductivity)

3.2 ELECTRICAL/MAGNETIC PROPERTIES (Elec. Resistivity, Mag. Resistivity, etc.)

3.3 MISCELLANEOUS (Viscosity, Density) 
4.0 CHEMICAL' PROPERTIES

5.0 NUCLEAR PROPERTIES

6.0 REFERENCES 


\subsection{BASIC DATA}

\subsection{GENERAL}

The outstanding characteristics of OFHC copper are its very high thermal and electrical conductivity, and its resistance to corrosion. It can be formed by most of the methods used for working metals. It is not resistant to sulfur compounds nor to high temerpature oxidation. It does resist most atmospheres, ground waters, and sea water. It can be joined by pressure welding and gas-shielded arc welding. It can be soft soldered or silver brazed.

\subsection{COMMERCIAL DESIGNATIONS}

Oxygen free copper O.F copper, and O.F.H. copper, oxygen-free with Silver.

Available Forms, Specifications, Suppliers

\begin{tabular}{|c|c|}
\hline FORMS AND CONDITIONS & SPECIFICATIONS \\
\hline Flat Products & $\begin{array}{l}\text { QQ-C-576 } \\
\text { ASTM-B8, B 124, B133, B152 (OF), } \\
\text { B } 187 \text { (OF), B272 }\end{array}$ \\
\hline Rods and Bars & $\begin{array}{l}Q Q-C-502 \\
\text { ASTM-B } 12-(O F), B 4 \dot{9}, B 124, \\
-B 133, \text { B } 187 \text { (OF) }\end{array}$ \\
\hline Wire & $\begin{array}{l}\text { ASTM-B 1-B2-B3-B33, } \\
\text { B189-B298 }\end{array}$ \\
\hline Tube & $\begin{array}{l}\text { ASTM-B-13 (OF), B68 (OF), } \\
\text { B75 (OF), B88, B } 111, \text { B } 188 \\
(O F), B 280\end{array}$ \\
\hline Pipe & ASTM B42 (OF), B188 (OF) \\
\hline Shapes & ASTM-B 124, -B 133, -Bp87 (OF) \\
\hline Welding Rods & $\begin{array}{l}\text { QQ-R -571 CLASS FSRCu-1, } \\
\text { MIL-R-19631 TYPE } \\
\text { FS-RCu-1 }\end{array}$ \\
\hline
\end{tabular}


1.3 CHEMICAL COMPOSITION (\%)

ELEMENT

MAXIMUM

MINIMUM

SOURCE ASTM B-170
Cu

99.95

\subsection{ROOM TEMPERATURE PROPERTIES}

(These properties are averages except for tensile ultimate strength, tensile yield strength, elongation, and reduction of area, which are $82 \%$ of average.)

\subsubsection{Mechanical Properties}

Tensile Ultimate Strength $F_{T}$

Annealed

$$
\begin{aligned}
& 1 / 4 \text { Hard } \\
& 1 / 2 \text { Hard }
\end{aligned}
$$

Hard

Spring Temper

TU

PSI

25,000

30,000

33,000

40,000

44,000

Tensile Yield Strength $\quad F_{\text {TY }}$

PSI

Anneal

$1 / 4$ Hard

$1 / 2$ Hard

Hard

Spring Temper

8,000

24,000

26,000

36,000

40,000

Comp. Yield Strength

$\mathrm{F}_{\mathrm{CY}}$

PSI

Shear Strength

$F_{\text {SU }}$

PSI

Ultimate Bearing Strength $F_{B R U}$

PSI 
Elongation

Annealed

1/4 Hard

I/2 Hard

Hard

Spring Temper

Reduction of Area

RA

E

G

6.4 e

$45 \%$

$25 \%$

$14 \%$

$6 \%$

$4 \%$
Modulus of Rigidity

Modulus of Elasticity $\quad 10^{6} \mathrm{PSI}$

17

G $\quad 10^{6}$ PSI

Comp. Modulus of Electicity $E_{C}$

$10^{6} \mathrm{PSI}$

\subsubsection{Physical Properties}

\begin{tabular}{lll}
\hline Density & $\rho$ & LB/IN \\
\hline & $0.321-0.323$
\end{tabular}

Specific Gravity

$$
8.89-8.94
$$

Specific Heat

c.

$B T U / L B-{ }^{\circ} F$

0.092

Thermal Conductivity

k

$\frac{B T U-F T}{H R-F T^{2}-F}$

\section{6}

Mean Coeff. Lin. Thermal Expans.

$10^{-6} \mathrm{IN}$.

$\left(32-572^{\circ} \mathrm{F}\right)$
Electrical Resistivity

$9.3-9.8$

IN. - OF

\subsection{4}




\subsubsection{Magnetic Properties}

Non-Magnetic

Melting Range

$$
1949^{\circ} \text { to } 1981^{\circ} \mathrm{F}
$$

Phase Changes

None

Thermal Conductivity VS. Temperature .

\begin{tabular}{|c|c|c|}
\hline $\begin{array}{l}\text { Temperature } \\
\text { (OF) }\end{array}$ & k & $\frac{B T U-F T}{H R-F T^{2}-O F}$ \\
\hline-100 & & 240 \\
\hline 68 & & 226 \\
\hline 900 & & 192 \\
\hline
\end{tabular}

Emissivity VS. Temperature

Between $1800^{\circ} \mathrm{F}$ and $2100^{\circ} \mathrm{F}$ Emissivity is:

$15 \%$ of Black Body for $7000 \&$ Light

$25 \%$ of Black Body for $6000 \AA$ Light

$37 \%$ of Black Body for 5500 \& Light

$45 \%$ of Black Body for $5000 \&$ Light

\subsubsection{Chemical Properties}

Copper resists the atmosphere, all notural fresh and salt waters, and alkaline solutions, except those containing ammonium ions. It also resists most organic compounds and all acids except the strong oxidizing acids, such as nitric and concentrated sulfuric acid when boiling. It resists hydrofluoric acid but not hydrochloric acid. It does resist dry chlorine but not wet chlorine. 


\subsubsection{Nuclear Properties}

TBD

\subsection{SPECIAL CONSIDERATIONS}

\section{Service Limits}

Copper is not very strong and the strengthening effect of cold working is removed by annealing at $500^{\circ} \mathrm{F}$.

Copper is not resistant to hydrochloric acid, nor is it resistant to wet chlorine. It is not resistant to nitric acid, cold or hot, nor is it resistant to boiling concentrated sulfuric acid. It does not resist high temperature oxidation. Silver is added to copper to increase the softening temperature and the creep resistance. It does not have a marked effect on the electrical conductivity giving a decrease in conductivity of about $0.15 \%$ for each ounce-per-ton of silver.

\section{I.6 PROCESSING}

\section{Surface Treatment}

As a general rule, no surface treatments are applied to copper.

Thermal Treatment

The only thermal treatment effective on copper is annealing after cold working. This reaches completion at temperatures as low as $500^{\circ} \mathrm{F}$.

\subsection{JOINING}

Copper can be joined with the silver brazing alloys, and with the lead tin solders. It can be welded by pressure welding and arc welded using the gas-shielded arc processes. See Reference 3. 


\subsection{FORMING}

Copper is very ductile and can be worked by any desired hot or cold method. Cold reductions of as much as $90 \%$ can be accomplished between anneals. See Reference 4 .

\subsection{MACHINABILITY}

Copper machines poorly, having a machinability index $20 \%$ of free-cutting brass. 


\subsection{MECHANICAL PROPERTIES}

2.1 SHORT TERM

2.1.1 Static

TENSILE PROPERTIES

Figure 2.1.1-1 Effect of Temperature on Drawing on Room

Temperature Tensile Strength of OFHC

Copper

Figure 2.1.1-2 Tensile Strengths

Figure 2.1.1-3 True Stress, Strain, Set and Offset

Corresponding to Various Yield Strength

Criteria

Table 2.1.1-1 Comparative Tensile Properties

Table 2.1.1-2 Effect of Cold Work on Tensile Properties

Table 2.1.1-3 Values of Stress, Strain, Set, and Offset

Corresponding to Various Yield Strength

Criteria (Ref. 6.9)

MODULUS OF ELASTICITY

Figure 2.1.1-4 Modulus of Flnsticity

HARDNESS

Figure 2.1.1-5 Effect of Cold Rolling on Indentation Hardness, Tensile Strength and Elongation of Oxygen

Free Copper

Table 2.1.1-4 Vickers Pyramid Hardness

Table 2.1.1-5 Hardness Data 


\section{ANNEALING, SOFTENING, RECRYSTALLIZATION}

Figure 2.1.1-6 Softening Curves for OFHC copper with Various Amounts of Cold Work

Figure 2.1.1-7 Tensile Strength of OFHC Copper as a

Function of Percent Reduction and

Temperature on Progressive Annealing

Figure 2.1.1-8 ' Uniform Elongation of OFHC Copper as a Function of Percent Reduction and Temperature of Exposure Reached on Progressive Annealing

Table 2.1.1-6 Softening Temperatures of OFHC Copper 


\section{TENSILE PROPERTIES}

Jenkins and Digges (6.6) give the tensile strength and yield strength of OFHC Copper as follows:
Tensile Strength Annealed
31,900 psi
Tensile Strength Cold-drawn $40 \%$
51,100 psi
Yield Strength Annealed
12,200 psi
Yield Strength Cold-drawn 40\%
50,300 psi

TABLE 2.1.1-1

Comparative Tensile Properties

\begin{tabular}{|l|l|c|}
\hline \multicolumn{1}{|c|}{ Property } & \multicolumn{2}{c|}{ lnitial Condition } \\
\cline { 1 - 3 } Cold Drawn \\
\hline Average grain diameter $\mathrm{mm}$ & Annealed & 40 Per Cent Reduction \\
\hline Average hardness Rockwell $\mathrm{F}$ & 0.025 & - \\
\hline Tensile strength $1000 \mathrm{psi}$ & 34 & 86 \\
\hline Yield strength 1000 psi & 31.9 & 51.1 \\
\hline Per cent elongation in 2 inches at maximum load & 39 & 50.3 \\
\hline Per cent elongation in 2 inches at fracture & 51 & 0.7 \\
\hline Reduction of area at maximum load & 28 & 11 \\
\hline Reduction of area at fracture & 88 & 0.68 \\
\hline
\end{tabular}

Tensile tests at a rate of extension approximately $1 \%$ per-minute. 
Benson, McKeown, and Mends (6.7) presented data showing the effect of cold-working on tensile properties of various coppers. The data with tons per square inch converted to psi are in Table 2.1.1.2.

TABLE 2.1.1-2

Effect of Cold Work on Tensile Properties

\begin{tabular}{|c|c|c|c|c|}
\hline \multirow{2}{*}{ Copper } & \multicolumn{4}{|c|}{ Cold Work Per Cent Reduction } \\
\hline & 0 & 10 & 25 & 50 \\
\hline & \multicolumn{4}{|c|}{$0.1 \%$ pre-strained (psi) } \\
\hline $\mathrm{OFHC}$ & 6720 & 30,200 & 39,000 & 47,500 \\
\hline Electrolytic tough pitch & 5152 & 29,800 & 38,750 & 45,900 \\
\hline OFHC + silver & 6272 & 30,250 & 37,630 & 47,250 \\
\hline $\begin{array}{l}\text { Electrolytic tough pitch } \\
+ \text { silver }\end{array}$ & 6720 & 30,700 & 39,200 & 47,700 \\
\hline & \multicolumn{4}{|c|}{ ultimate tensile strength (psi) } \\
\hline OFHC & 32,250 & 35,170 & 44,130 & 47,490 \\
\hline Electrolytic tough pitch & 30,900 & 36,500 & 45,250 & 45,920 \\
\hline OFHC + silver & 31,580 & 34,950 & 44,800 & 47,260 \\
\hline \multirow[t]{2}{*}{$\begin{array}{l}\text { Electrolytic tough pitch } \\
+ \text { silver }\end{array}$} & 32,480 & 35,840 & 45,000 & 47,700 \\
\hline & \multicolumn{4}{|c|}{ elongation (per cent) } \\
\hline OFHC & 72 & 44 & 23 & 12 \\
\hline Electrolytic tough pitch & 68 & 41 & 19 & 14 \\
\hline OFHC + silver & 72 & 52 & 15 & 13 \\
\hline $\begin{array}{l}\text { Electrolytic tough pitch } \\
+ \text { silver }\end{array}$ & 65 & 46 & 19 & 12 \\
\hline
\end{tabular}


According to Ellis and Greiner (6.8), there is a marked difference in the tensile properties of OFHC copper when drawn at different temperatures. Figure 2.1.1-3 is adapted from their paper.

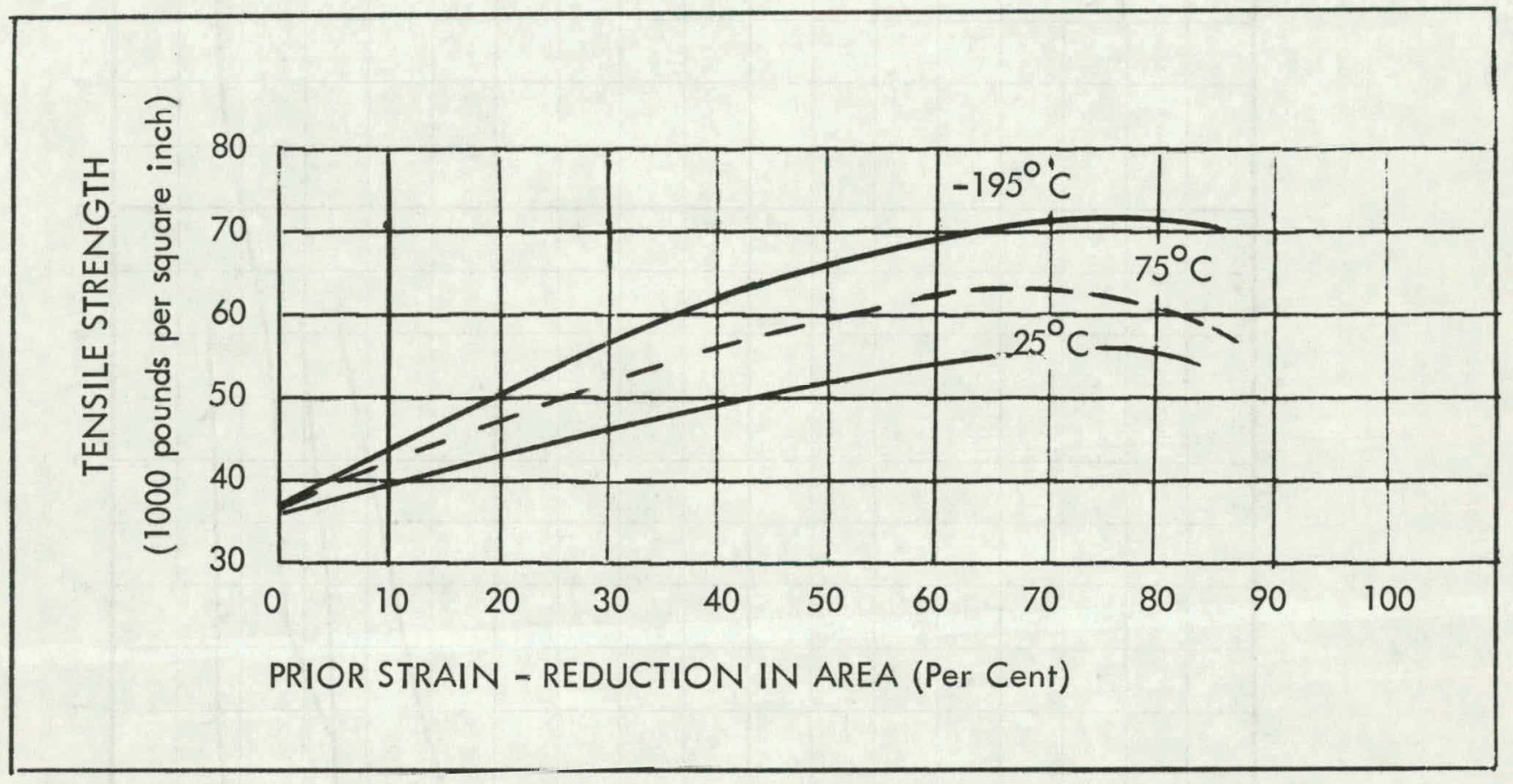

Figure 2.1.1-1 Effect of temperature of drawing on room-temperature tensile strength of OFHC copper.

After Ellis and Greiner (Ref. 6.8). 


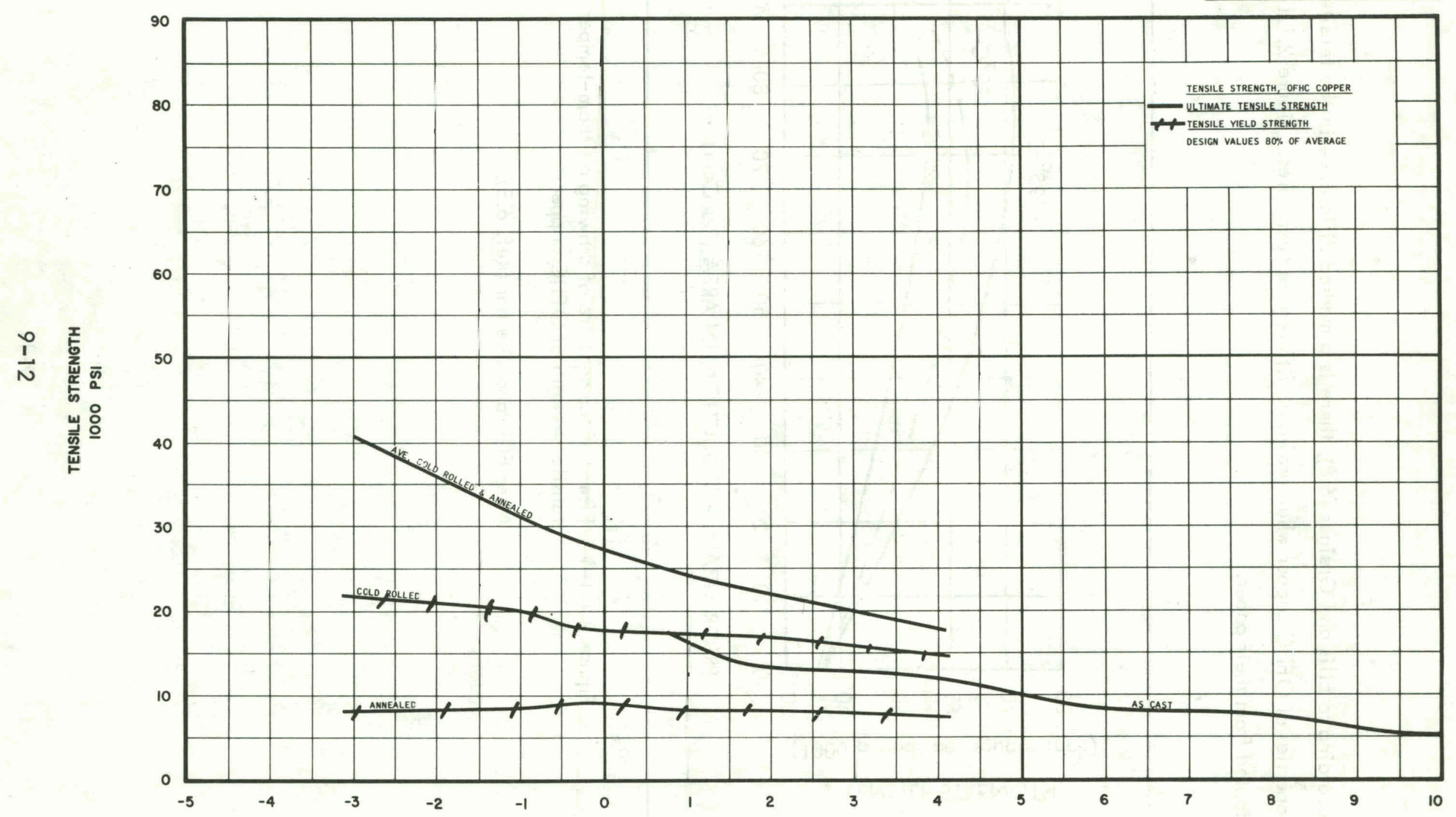

Figure 2.1.1-2 
TABLE 2.1.1-3

Values of Stress, Strain, Set, and Offset Corresponding to Various Yield Strength Criteria (Ref. 6.9)

\begin{tabular}{|c|c|c|c|c|c|c|c|c|}
\hline \multirow{2}{*}{ Copper } & \multicolumn{3}{|c|}{$\begin{array}{l}0.5 \text { Per Cent } \\
\text { Total Strain }\end{array}$} & \multirow{2}{*}{ 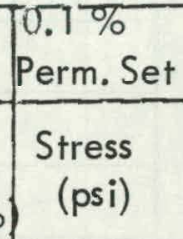 } & \multicolumn{2}{|c|}{0.1 Per Cent Offset } & \multicolumn{2}{|c|}{ 0.2 Per Cent Offset } \\
\hline & $\begin{array}{l}\text { Stress } \\
\text { (psi) }\end{array}$ & $\begin{array}{l}\text { Offset } \\
(\%)\end{array}$ & $\begin{array}{l}\text { Res. } \\
\text { Perm. } \\
\text { Set(\% }\end{array}$ & & $\begin{array}{l}\text { Stress } \\
\text { (psi) }\end{array}$ & $\begin{array}{c}\text { Total } \\
\text { Strain(\%) }\end{array}$ & $\begin{array}{r}\text { Stress } \\
(p s i)\end{array}$ & $\begin{array}{c}\text { Total } \\
\text { Strain (\%) }\end{array}$ \\
\hline Electrolytic & 46,000 & 0.229 & 0.212 & 44,300 & 43,700 & 0.359 & 45,700 & 0.473 \\
\hline OFHC & 47,000 & 0.232 & 0.222 & 45,000 & 44,600 & 0.355 & 46,800 & 0.468 \\
\hline
\end{tabular}

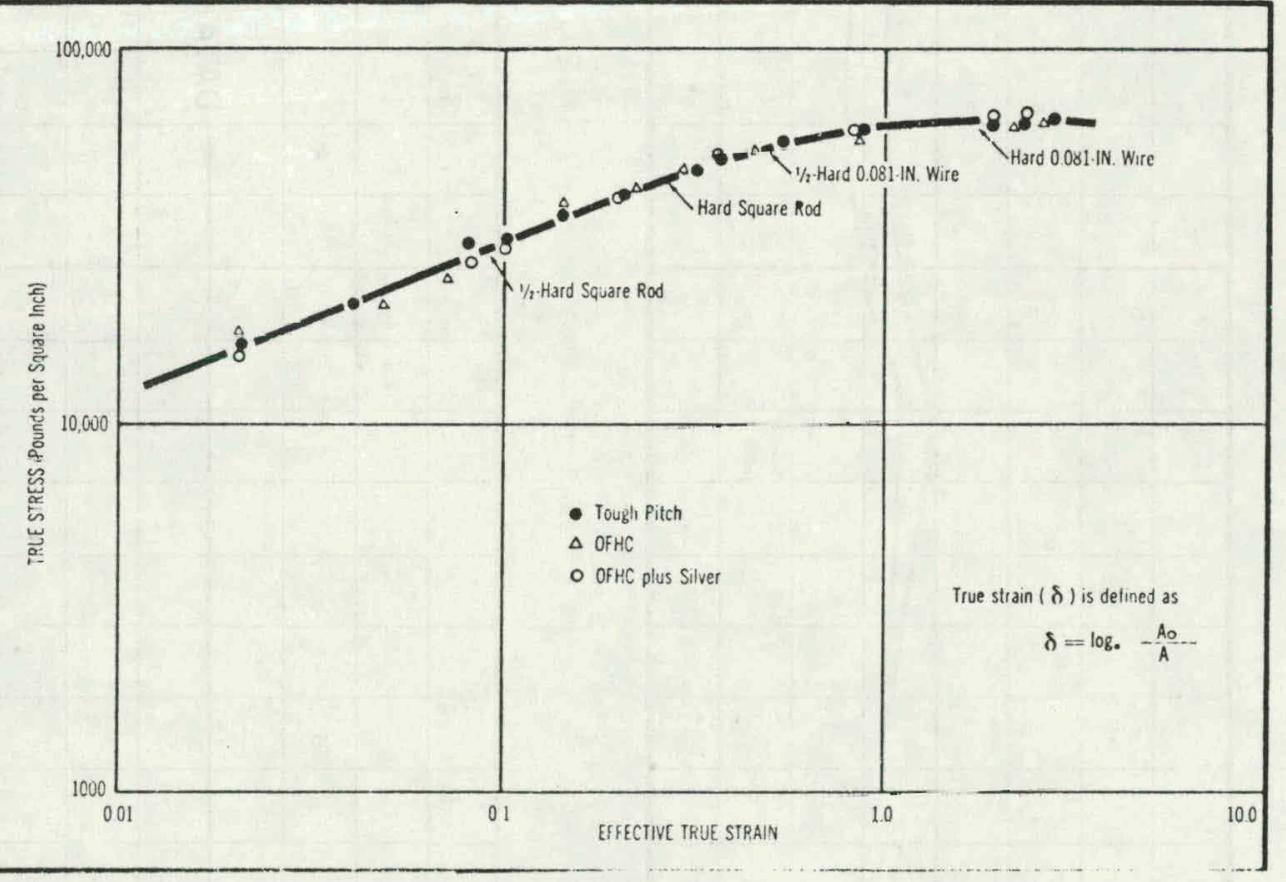

Figure 2.1.1-3 True stress-strain curves for tough pitch, OFHC, and OFHC plus silver (Ref. 6.10). 
MODULUS OF ELASTICITY
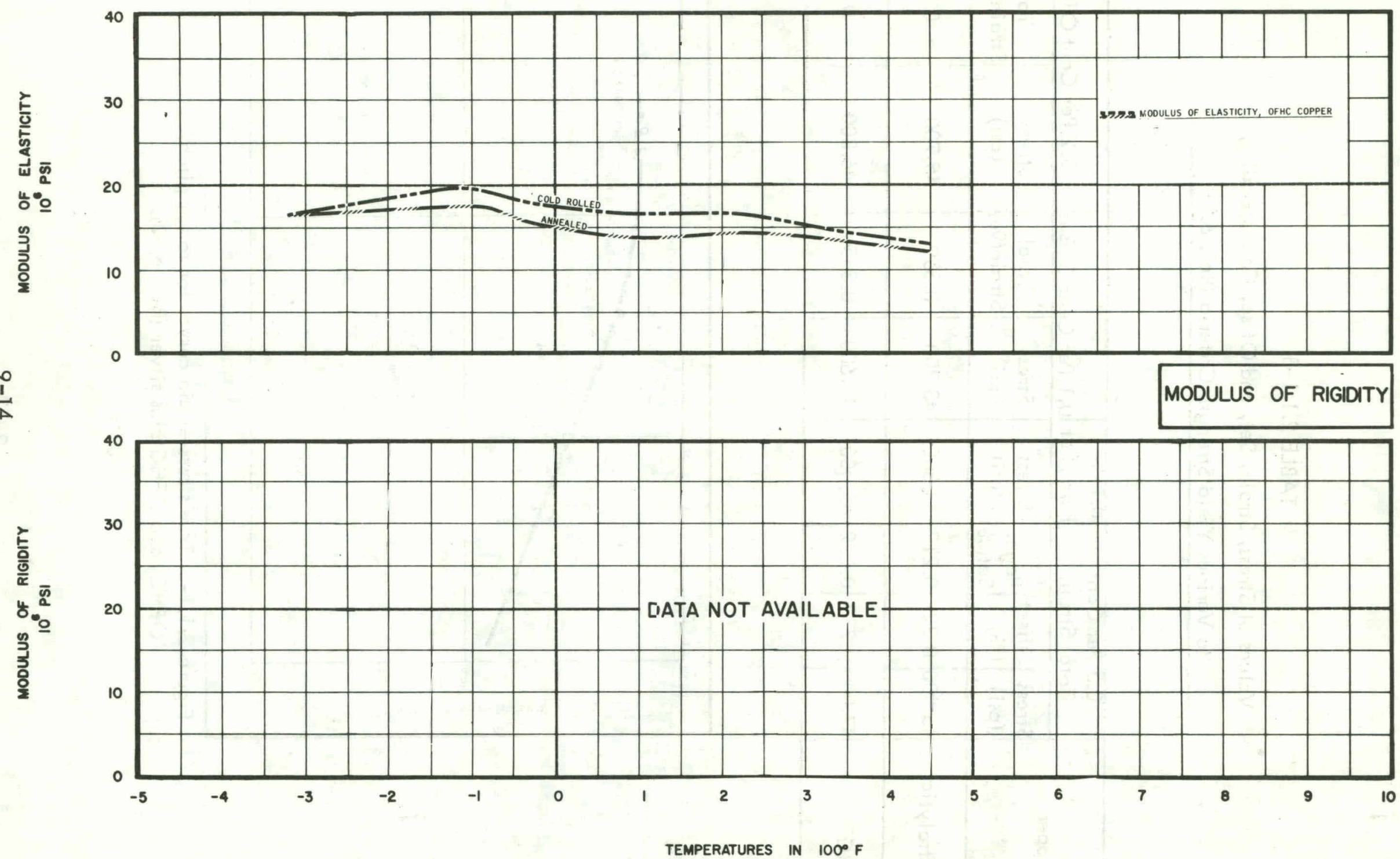

Figure 2.1.1-4 


\section{HARDNESS}

Table 2.1.1-5 is a compilation of hardness data chiefly from the literature.

The authors of Ref. 6.7 determined the effect of cold-working on hardness. The data are in Table $2.1 .1-4$.

Thompson (6.12) studied the indentation hardness (and other properties) as affected by colding-working. The direct work reduction (by rolling) was taken far beyond commercial practice. Figure 2,1.1-5 is adapted from the paper.

'TABLE 2.1.1-4

Vickers Pyramid Hardness

\begin{tabular}{|c|c|c|c|c|}
\hline $\begin{array}{c}\text { Cold Work } \\
\text { (per cent) }\end{array}$ & $\begin{array}{l}\text { Tough } \\
\text { Pitch }\end{array}$ & OFHC & $\begin{array}{l}\text { Tough Pitch } \\
+ \text { Silver }\end{array}$ & $\begin{array}{l}\text { OFHC } \\
+ \text { Silver }\end{array}$ \\
\hline 0 & 48.0 & 36.8 & 44.9 & 37.8 \\
\hline 10 & 94.1 & 91.0 & 96.1 & 93.4 \\
\hline 25 & 105.0 & 101.3 & 105.0 & 101.0 \\
\hline 50 & 114.5 & 111.0 & 116.0 & 115.0 \\
\hline
\end{tabular}


TABLE 2.1.1-5

Hardness Data

\begin{tabular}{|c|c|c|c|c|c|c|c|c|}
\hline \multirow[b]{2}{*}{ Copper } & \multirow[b]{2}{*}{ Shape } & \multirow{2}{*}{$\begin{array}{c}\text { Per Cent } \\
\text { Work Re- } \\
\text { duction } \\
\end{array}$} & \multicolumn{2}{|c|}{ Annealed } & \multirow[b]{2}{*}{ Method } & \multicolumn{2}{|c|}{ Hardness } & \multirow[b]{2}{*}{ Reference } \\
\hline & & & $\begin{array}{l}\text { Time } \\
\text { (hrs) }\end{array}$ & $\begin{array}{c}\text { Temperature } \\
\left({ }^{\circ} \mathrm{C}\right)\end{array}$ & & Ann. & Hard & \\
\hline \multirow{2}{*}{ OFHC } & Wire & 75 & 2 & 300 & $R_{F}$ & 30 & 89 & + \\
\hline & Rod & 40 & & & & & & \\
\hline OFHC & Rod & 50 & 1 & 600 & V & 40 & 114 & \multirow{3}{*}{3} \\
\hline $\begin{array}{l}\text { Tough Pitch } \\
\text { No. } 1\end{array}$ & Rod & 50 & 1 & 600 & V & 40 & 116 & \\
\hline $\begin{array}{l}\text { Tough Pitch } \\
\text { No. } 2\end{array}$ & Rod & 50 & 1 & 600 & V & 40 & 115 & \\
\hline OFHC & $\begin{array}{l}\text { Annealed } \\
\text { Rod }\end{array}$ & & & & $H_{B}$ & 42 & & \multirow{6}{*}{66} \\
\hline OFHC & $\begin{array}{l}\text { Annealed } \\
\text { Rod }\end{array}$ & & & & V & 51 & & \\
\hline OFHC & $\begin{array}{l}\text { Annealed } \\
\text { Rod }\end{array}$ & 15 & & & $H_{B}$ & & 82 & \\
\hline OFHC & $\begin{array}{l}\text { Annealed } \\
\text { Rod }\end{array}$ & 15 & & & V & & 90 & \\
\hline OFHC & $\begin{array}{l}\text { Annealed } \\
\text { Rod }\end{array}$ & 30 & & & $H_{B}$ & & 96 & \\
\hline OFHC & $\begin{array}{l}\text { Annealed } \\
\text { Rod }\end{array}$ & 30 & & & V & & 106 & \\
\hline OFHC & Strip & 0 & & & V & 37 & & \multirow{8}{*}{7} \\
\hline OFHC & Strip & 10 & & & V & & 91 & \\
\hline OFHC & Strip & 25 & & & V & & 101 & \\
\hline OFHC & Strip & 50 & & & $\mathrm{~V}$ & & 111 & \\
\hline $\mathrm{OFHC}+\mathrm{Ag}$ & Strip & 0 & & & V & 38 & & \\
\hline $\mathrm{OFHC}+\mathrm{Ag}$ & Strip & 10 & & & V & & 93 & \\
\hline $\mathrm{OFHC}+\mathrm{Ag}$ & Strip & 25 & & & V & & 102 & \\
\hline $\mathrm{OFHC}+\mathrm{Ag}$ & Strip & 50 & & & V & & 115 & \\
\hline Tough Pitch & Strip & 0 & & & $\mathrm{~V}$ & 48 & & \multirow{4}{*}{7} \\
\hline Tough Pitch & Strip & 10 & & & V & & 94 & \\
\hline Tough Pitch & Strip & 25 & & & V & & 105 & \\
\hline Tough Pitch & Strip & 50 & & & V & & 115 & \\
\hline
\end{tabular}


TABLE 2.1.1-5 (Continued)

Hardness Data

\begin{tabular}{|c|c|c|c|c|c|c|c|c|}
\hline \multirow[b]{2}{*}{ Copper } & \multirow[b]{2}{*}{ Shape } & \multirow{2}{*}{$\begin{array}{l}\text { Per Cent } \\
\text { Work Re- } \\
\text { duction }\end{array}$} & \multicolumn{2}{|c|}{ Annealed } & \multirow[b]{2}{*}{ Method } & \multicolumn{2}{|c|}{ Hardness } & \multirow[b]{2}{*}{ Reference } \\
\hline & & & \begin{tabular}{|c|} 
Time \\
(hrs)
\end{tabular} & $\begin{array}{c}\text { Temperature } \\
\left({ }^{\circ} \mathrm{C}\right)\end{array}$ & & Ann. & Hard & \\
\hline Tough Pitch $+\mathrm{Ag}$ & Strip $^{*}$ & 0 & & & V & 45 & & \multirow{4}{*}{7} \\
\hline Tough Pitch $+\mathrm{Ag}$ & Strip ${ }^{*}$ & 10 & & & V & & 96 & \\
\hline Tough Pitch $+\mathrm{Ag}$ & Strip ${ }^{*}$ & 25 & & & V & & 105 & \\
\hline Tough Pitch $+\mathrm{Ag}$ & Strip ${ }^{*}$ & 50 & & & V & & 116 & \\
\hline OFHC & Tubing & 62.5 & & & $R_{F}$ & & 94 & \multirow{6}{*}{15} \\
\hline OFHC & Tubing & 90.5 & & & $R_{F}$ & & 96 & \\
\hline $\begin{array}{l}\text { OFHC +P } \\
\text { (Amphos) }\end{array}$ & Tubing & 62.5 & & & $R_{F}$ & & 96 & \\
\hline $\begin{array}{l}\text { OFHC + P } \\
\text { (Amphos) }\end{array}$ & Tubing & 90.5 & & & $R_{F}$ & & 96.5 & \\
\hline $\begin{array}{l}\text { Phosphorus- } \\
\text { Deoxidized }\end{array}$ & Tubing & 62.5 & & & $R_{F}$ & & 96 & \\
\hline $\begin{array}{l}\text { Phosphorus- } \\
\text { Deoxidized }\end{array}$ & Tubing & 90.5 & & & $R_{F}$ & & 98.5 & \\
\hline
\end{tabular}

${ }^{x} .1-1 / 4$ inch $\times 0.1$ inch $\quad R_{F}=$ Rockwell $F \quad V=$ Vịckers $\quad$ Data from AMCO Laboratory 
THICKNESS (Inches)

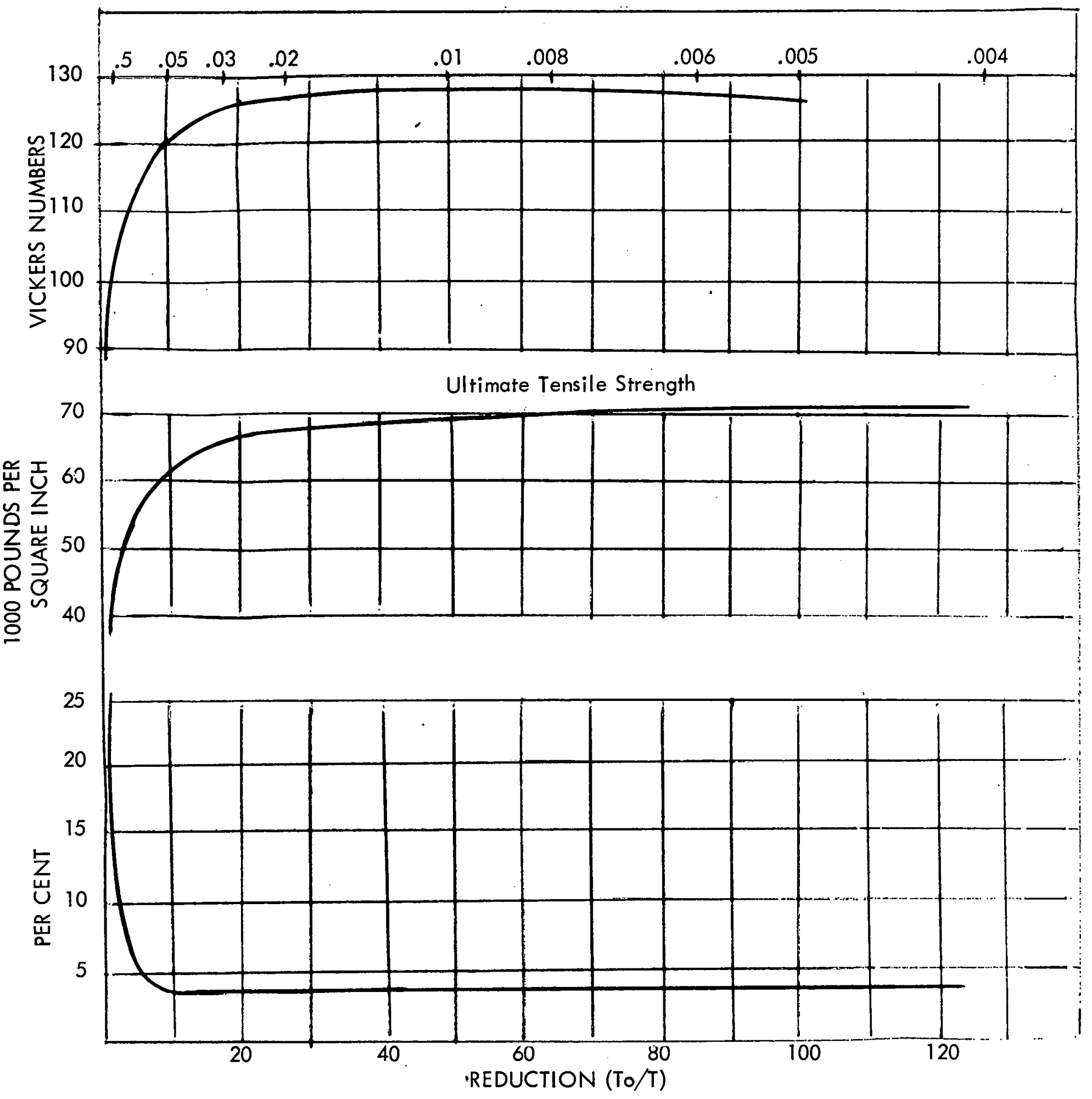

Figure 2.1.1-5 Effect of cold rolling on indentation hardness, tensile strength, and elongation of oxygen-free copper. 


\section{ANNEALING, SOFTENING, RECRYSTALLIZATION}

The response of copper to thermal treatment depends not only on its composition but to a considerable extent on the prior treatment. Thus, for a proper presentation of the results of heating tests, the history of the specimens should be given.

Since 1957, the impurity content of OFHC copper has been materially decreased. This means less contaminating material in solid solution, and therefore softening takes place at a lower temperature than before.

According to tests made by $A M C O$, the softening temperatures for recent production of OFHC coppers are as shown in Table 2.1.1-6.

TABLE 2.1.1-6

Softening Temperatures of OFHC Copper ;

\begin{tabular}{|l|c|c|c|}
\hline Copper & Cold Work & $\begin{array}{c}\text { Penultimate } \\
\text { Anneal }\end{array}$ & $\begin{array}{c}\text { Temperature } \\
\text { 1/2 Softened }\end{array}$ \\
\hline OFHC & 62 & $600^{\circ} \mathrm{C}$ & $225^{\circ} \mathrm{C}$ \\
\hline OFHC & 75 & $600^{\circ} \mathrm{C}$ & $218^{\circ} \mathrm{C}$ \\
\hline OFHC & 94 & $600^{\circ} \mathrm{C}$ & $208^{\circ} \mathrm{C}$ \\
\hline OFHC & 94 & $850^{\circ} \mathrm{C}$ & $240^{\circ} \mathrm{C}$ \\
\hline OFHC & 62 & $900^{\circ} \mathrm{C}$ & $250^{\circ} \mathrm{C}$ \\
\hline Amphos (OFHC $+\mathrm{P})$ & 94 & $600^{\circ} \mathrm{C}$ & $296^{\circ} \mathrm{C}$ \\
\hline $\begin{array}{l}\text { Electrolytic } \\
\text { tough pitch }\end{array}$ & 94 & $600^{\circ} \mathrm{C}$ & $218^{\circ} \mathrm{C}$ \\
\hline
\end{tabular}

For comparison 


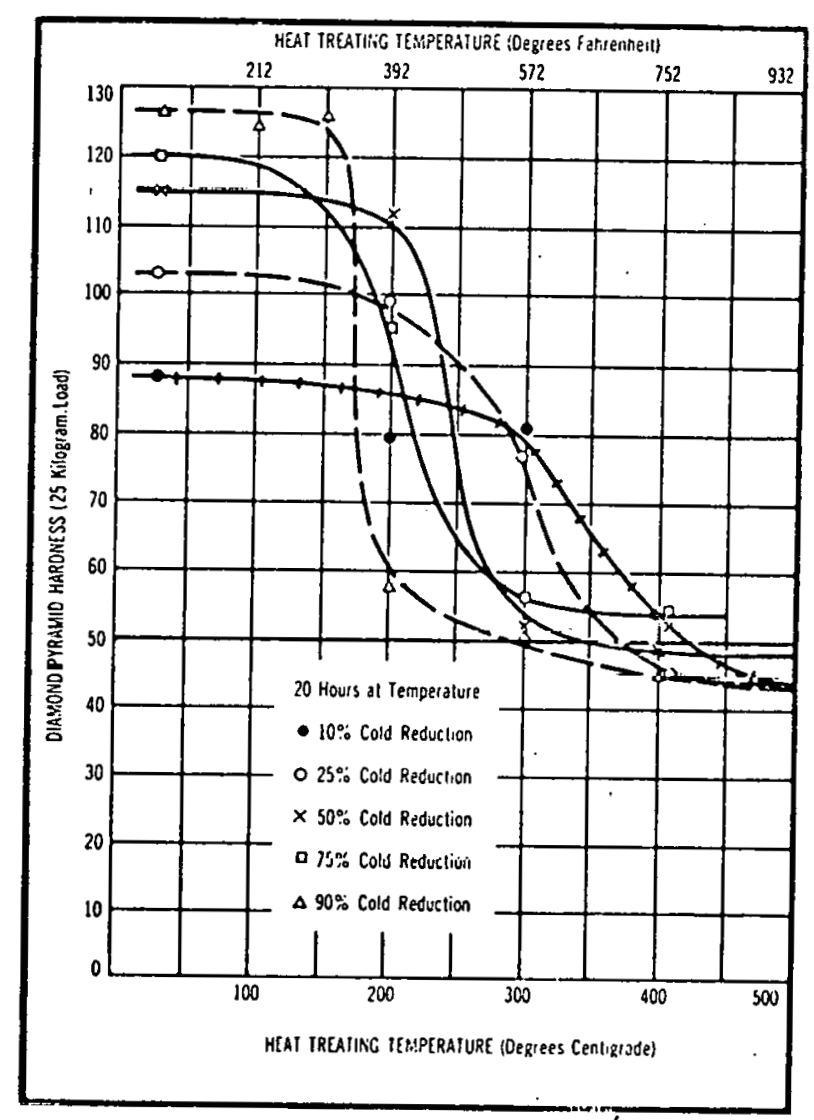

Figure 2.1.1-6 Softening curves for OFHC copper with various amounts of cold work. 
Yokelson and Balicki (6.29) made an extensive study of the work-hardening and reannealing characteristics of five brands of high-conductivity copper, including OFHC, in order to establish the relative thermal stability in the work-hardened condition. They used the spectrosopically pure copper as a standard.

Figure 2.1.1-7 and 2.1.1-8 are reproduced from this paper. They are space-diagrams showing the interrelationship between work-hardening, temperature of anneal, and tensile strength for OFHC copper. 


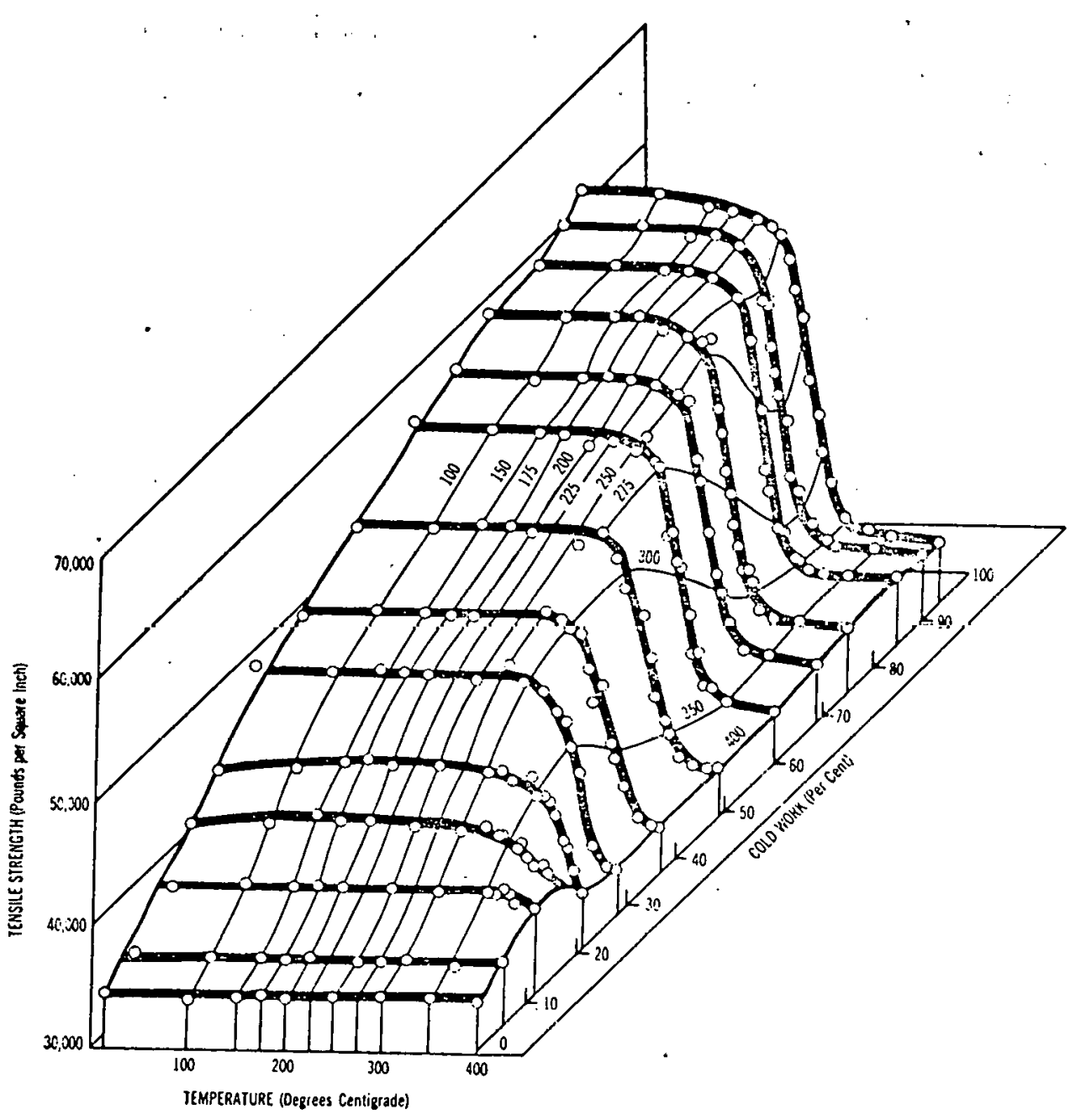

Figure 2.1.1-7 Tensile strength of OFHC copper as a function of per cent reduction and temperature on progressive reannealing. Heating rate employed, $10.8^{\circ} \mathrm{C}$ per minute. 


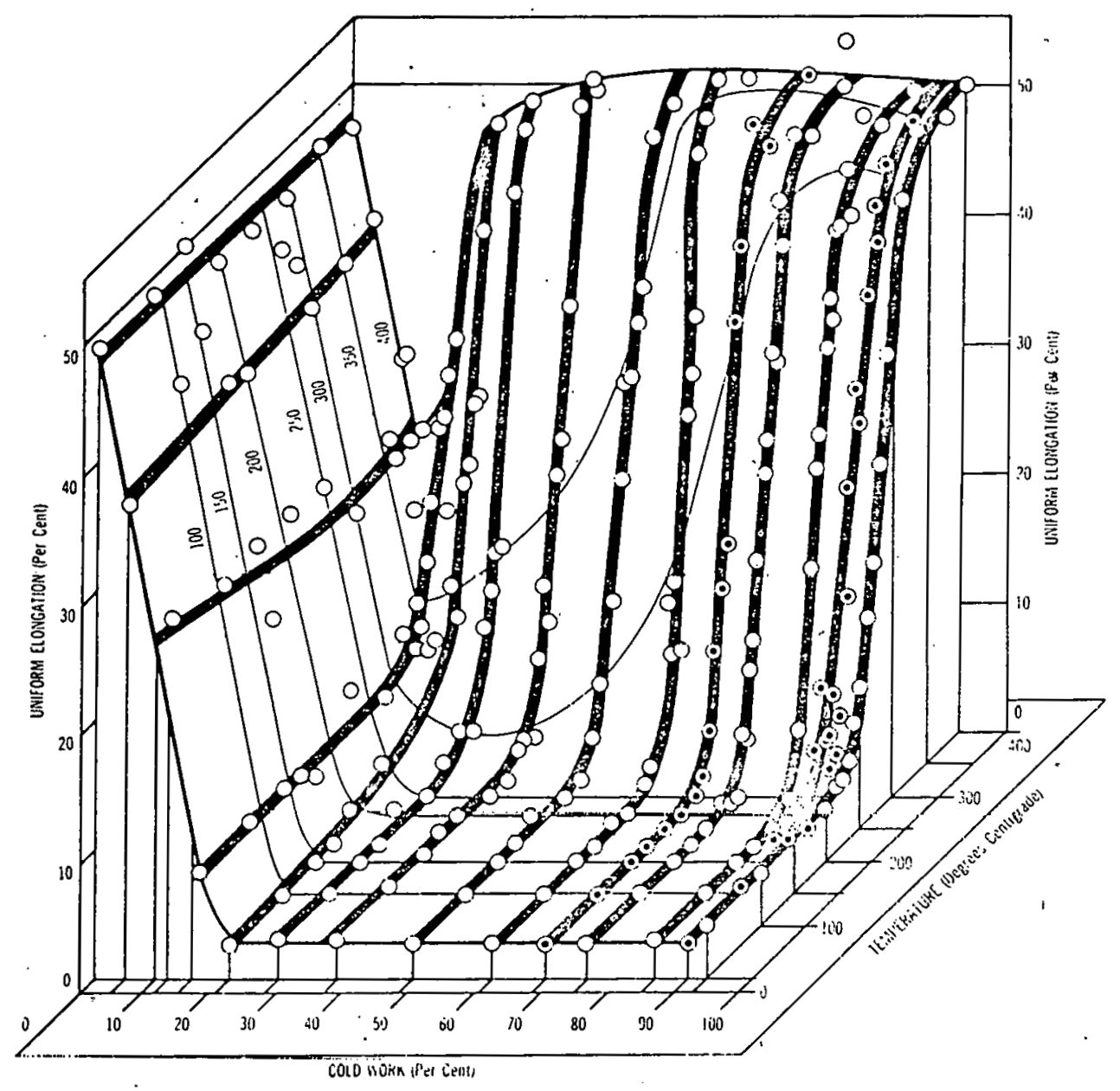

Figure 2.1.1-8 Uniform elongation of OFHC copper as a function of per cent reduction and temperature of exposure reached on progressive reannealing. Heating rate employed, $10.8^{\circ} \mathrm{C}$ per minute. 


\subsubsection{Dynamic}

IMPACT

Figure 2.1.2-1

Figure 2.1.2-2

Table 2.1.2-1
Impact Strength and Reduction of Area

Impact versus Hardness

Impact Test Data 
IMPACT STRENGTH

ROCKWELL HARDNESS

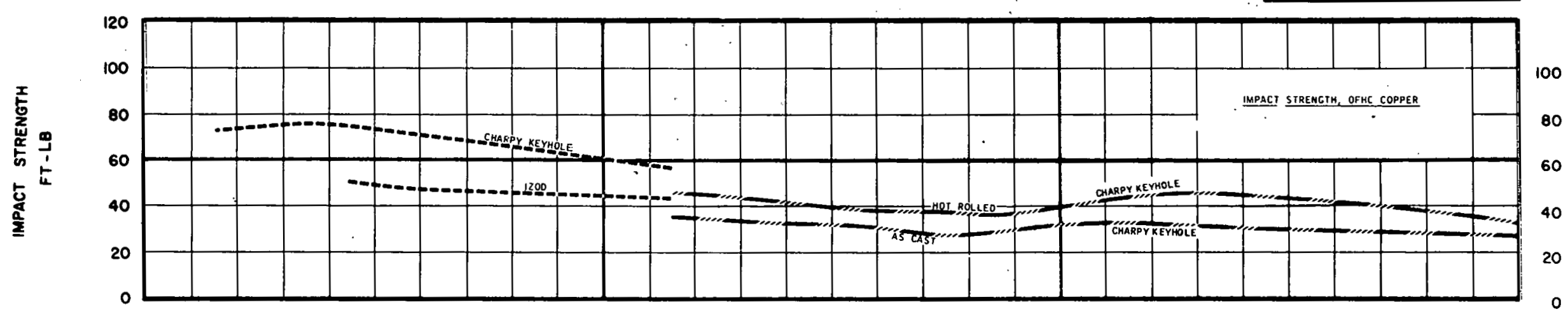

نั

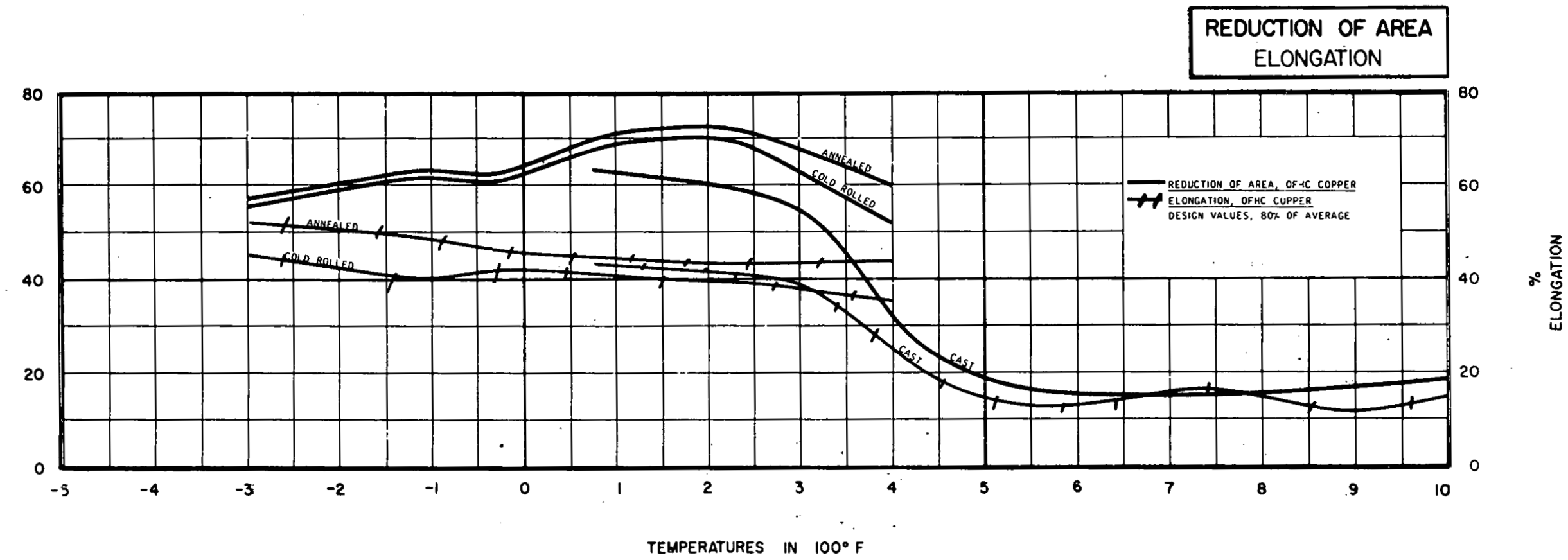

Figure 2.1.2-1 


\section{IMPACT STRENGTH}

In all test results found in the literature, the data show a marked superiority for the oxygen-free copper in impact strength. According to Lorig, Dahle, and Roberts (6.12), the addition of phosphorus to OFHC copper causes no change in the impact strength at room temperature and only a small increase at higher temperatures.

Miller (6.13) mentions two papers in which the adverse effect of oxygen on impact strength of copper is indicated.

Rolle, in his discussion of Corson (6.14), cites data showing that phosphorus-deoxidized copper, though stronger than tough pitch, is not quite up to the strength of OFHC copper. It is interesting to note that, according to Rolle, hardening the OFHC and phosphorus-deoxidized coppers results in higher impact values, whereas for the tough-pitch (oxygen-bearing) copper the reverse is indicated.

The data submitted by Rolle are based on the following conditions:

All tests were made on 1/2-inch-square rods of wrought copper. All annealing was at $450^{\circ} \mathrm{C}$ for 60 minutes, in air. The phosphorized copper that was tested was produced by deoxidation with phosphorus. Impact data are averages of 15 determinations. Two sets of tests were made. Rolle's data are presented in Table 2.1.11 and Figure 2.1.11. 
TABLE 2.1.2-1

Impact Test Data

\begin{tabular}{|l|c|c|c|c|c|}
\hline Copper & $\begin{array}{c}\text { Tensile } \\
\text { Strength } \\
\text { (psi) }\end{array}$ & $\begin{array}{c}\text { Reduc- } \\
\text { tion } \\
\text { of area } \\
\text { (per cent) }\end{array}$ & $\begin{array}{c}\text { Elonga- } \\
\text { tion } \\
\text { in } 8 \text { in. } \\
\text { (per cent) }\end{array}$ & $\begin{array}{c}\text { Brinell } \\
\text { Hardness }\end{array}$ & $\begin{array}{c}\text { Izod } \\
\text { Impact } \\
\mathrm{Ft}-\mathrm{Lb}\end{array}$ \\
\hline Hard OFHC & 46,000 & 89 & 5.0 & 92 & 73.2 \\
\hline Hard ETP $^{*}$ & 48,100 & 57 & 4.0 & 91 & 35.4 \\
\hline Annealed OFHC $^{*} 31,900$ & 91.9 & 43.5 & 53 & 54.1 \\
\hline Annealed ETP $^{*}$ & 32,000 & 72.5 & 43.5 & 54 & 48.5 \\
\hline Hard OFHC $^{\text {Hard Phos. }}{ }^{+}$ & 51,450 & 88.3 & 5.0 & 96 & 71.7 \\
\hline Annealed OFHC & 32,000 & 91.5 & 42.0 & 67 & 61.3 \\
\hline Annealed Phos. & 33,200 & 83.7 & 39.6 & 69 & 57.3 \\
\hline
\end{tabular}

The data for izod impact, when plotted against Brinell hardness, the two points in each case being connected with a straight line, give results as shown in Figure 2.1.2-2. 


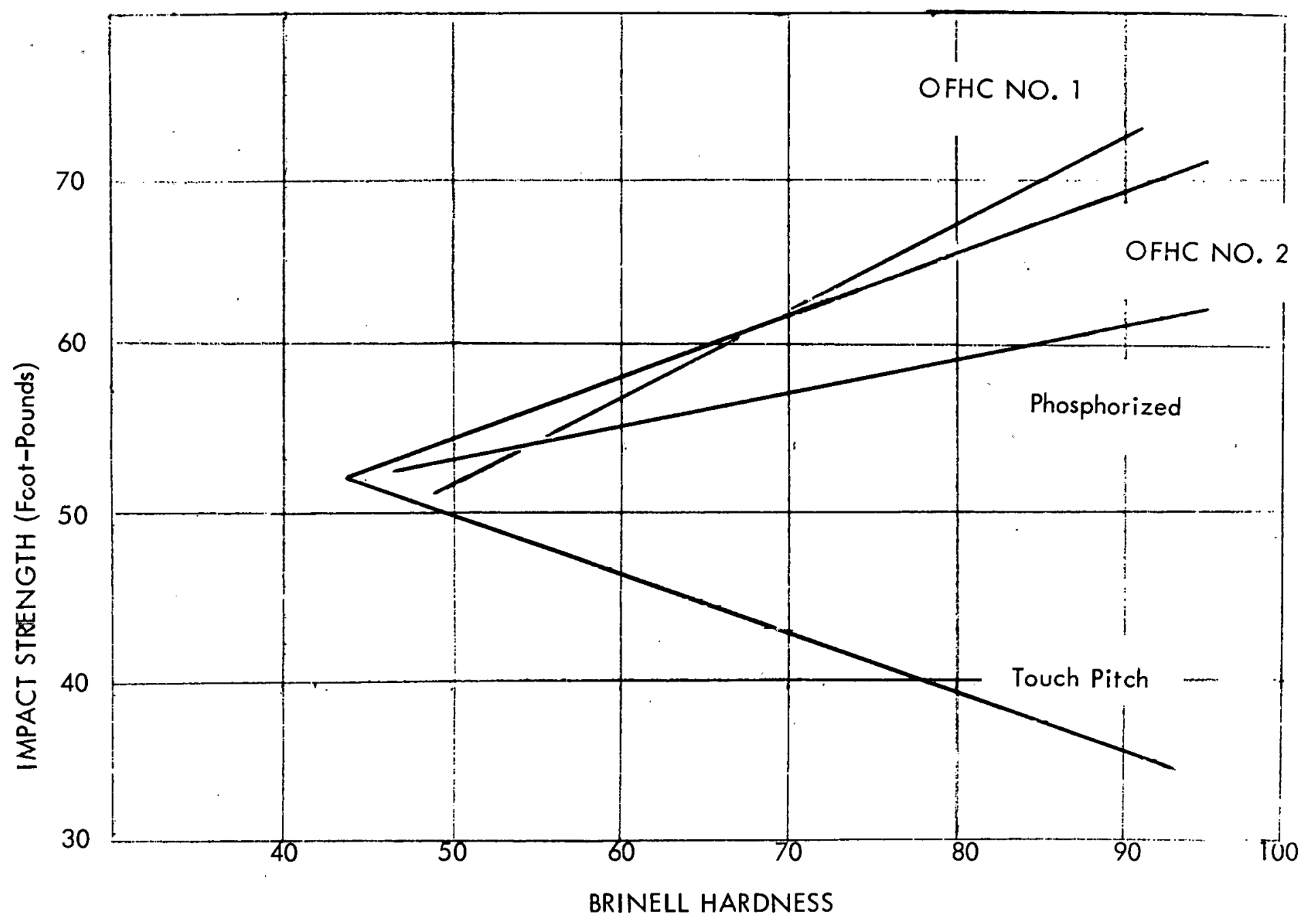

Figure 2.1.2-2 Impact Versus Hardness. 
2.2 LONG TERM

CREEP AND RUPTURE

Figure 2.2-1

Table 2.2-1

Table 2.2-2

Table 2.2-3
Temperature Stress Limits Curves for

OFHC and OFHC plus Silver, for Various

Creep Rates

Creep Stress Limits

Creep Stress Limits

Creep Stress Limits (Long Duration Tests) 


\section{CREEP AND RUPTURE}

Many papers have been published on creep and on variations on that theme in which the tests reported were made on OFHC copper.

The following references are pertinent:

Schwope, Smith, and Jackson (6.15). The following summary is from this paper:

The effect of cold work on the creep characteristics of tough-pitch and OFHC coppers, unalloyed and silver-bearing, has been determined for temperatures from $200\left(93^{\circ} \mathrm{C}\right)$ to $572^{\circ} \mathrm{C}$. The most important results are:

1. Cold work increases the creep strength of copper; however, the benefit from cold work is lost at temperatures where recrystallization is rapid. This temperature varies with the amount of cold work and the type of copper.

2. The addition of silver to either tough-pitch or OFHC copper raises the temperature at which rapid recrystallization occurs; the effect is approximately the same on both types of copper.

3. While additions of silver effectively lower the creep rate of both touch-pitch and OFHC copper as cold-worked, the silver-bearing OFHC copper has a markedly lower creep rate than comparable tough-pitch copper.

Vosskuhler (6.16). Vosskuhler devoted a great deal of time and effort to correlating the results reported in all the references he had available on the subject of creep. in copper 
and dilute copper alloys. Thus he put all the data on a common basis, permitting direct comparison. He covered also tests taken to rupture.

Tables 2.2-1, 2.2-2, and 2.2-3 are from Vosskuhler's paper, with kilograms per square mill imeter converted to pounds per square inch. In any interpretation of the data it must be noted that there was a considerable variation in the various factors involved in the tests in the various references. Among such variables are preparation, pretreatment, size and shape of specimens, and the method and conditions of the testing.

Vosskuhler assembled all the data available for OFHC and OFHC plus silver, and by plotting on double logarithmic paper derived a relationship between creep rate and temperature which he then plotted linearly. His data have been replotted to show the stresses in pounds per square inch instead of kilograms per square millimeter. The revised curves are shown in Figure 2.2-1. 
TABLE 2.2-1

Creep Stress Limits

Data from Reference 6.27

\begin{tabular}{|c|c|c|c|c|c|c|}
\hline \multirow[b]{2}{*}{ Material } & \multirow[b]{2}{*}{ Condition . } & \multicolumn{2}{|c|}{ Temperature } & \multicolumn{3}{|c|}{$\begin{array}{l}\text { Creep Stress Limit } \\
\text { (psi) }\end{array}$} \\
\hline & & ${ }^{\circ} \mathrm{C}$ & ${ }^{o} F$ & $0.001 \%$ & $\begin{array}{l}\text { Per } 1000 \mathrm{Hrs} \\
0.010 \%\end{array}$ & $0.1 \%$ \\
\hline \multirow{6}{*}{$\mathrm{Cu}+\mathrm{O}_{2}$} & Annealed & 150 & 300 & 1100 & $2950^{\circ}$ & 8000 \\
\hline & \multirow{2}{*}{$\begin{array}{l}\text { Grain } \\
0.025 \mathrm{~mm}\end{array}$} & 205 & 400 & 400 & 1300 & 4000 \\
\hline & & 260 & 500 & 100 & 350 & 1150 \\
\hline & \multirow{2}{*}{ Drawn' $84 \%$} & 150 & & 5000 & 9400 & 14700 \\
\hline & & 205 & & 1000 & 2100 & 4350 \\
\hline & Annealed & 150 & & 1600 & 3600 & 8200 \\
\hline \multirow{4}{*}{ OFHC } & \multirow{2}{*}{$\begin{array}{l}\text { Grain } \\
0.025 \mathrm{~mm}\end{array}$} & 205 & & 500 & 1500 & 4800 \\
\hline & & 260 & & 100 & 400 & 1700 \\
\hline & \multirow{2}{*}{ Drawn $84 \%$} & 150 & & & 8300 & 12800 \\
\hline & & 205 & & 570 & 1750 & 5000 \\
\hline
\end{tabular}


TABLE 2.2-2

Creep Stress Limits

Data* from Reference 6.15

\begin{tabular}{|c|c|c|c|c|c|}
\hline \multirow[b]{2}{*}{ Material } & \multirow{2}{*}{ Condition } & \multicolumn{2}{|c|}{$\begin{array}{c}\text { Creep Stress } \\
(p s i)\end{array}$} & \multirow{2}{*}{$\begin{array}{l}\text { Tensile } \\
\text { Strength } \\
\text { (psi) }\end{array}$} & \multirow[b]{2}{*}{$\begin{array}{l}\text { Elongation } \\
\text { (per cent) }\end{array}$} \\
\hline & & $\begin{array}{c}0.001 \% \\
\text { per hr }\end{array}$ & $\begin{array}{l}0.01 \% \\
\text { per hr }\end{array}$ & & \\
\hline \multirow{2}{*}{ OFHC } & Drawn 5\% & 4300 & 13,000 & 33,000 & 36 \\
\hline & Rolled 5\% & 4750 & 14,400 & 33,100 & 35 \\
\hline \multirow{4}{*}{$\begin{array}{c}\mathrm{OFHC}+\mathrm{Ag} \\
0.054 \%\end{array}$} & Rolled 5\% & 6000 & 16,100 & 33,200 & 36 \\
\hline & Rolled $20 \%$ & 2200 & 12,500 & 45,500 & 2 \\
\hline & Rolled $30 \%$ & 1850 & 11,800 & 46,750 & 3 \\
\hline & Rolled $40 \%$ & 1850 & & 50,750 & 2.5 \\
\hline $\begin{array}{c}\mathrm{OFHC}+\mathrm{Ag} \\
0.076 \% \\
\end{array}$ & Rolled 5\% & 7150 & 17,250 & 33,100 & 35 \\
\hline \multirow{4}{*}{$\begin{array}{r}\mathrm{Cu}+\mathrm{O}_{2} \\
0.063 \%\end{array}$} & Rolled $5 \%$ & 1750 & 9,700 & 36,400 & 33 \\
\hline & Rolled $20 \%$ & 1140 & 7,550 & 45,400 & 3.5 \\
\hline & Rolled $30 \%$ & 1140 & 7,850 & 48,800 & 3 \\
\hline & Rolled $40 \%$ & 715 & 6,400 & 51,000 & 2.5 \\
\hline \multirow{2}{*}{$\begin{array}{l}\mathrm{Cu}+\mathrm{O}_{2} \\
+0.072 \% \mathrm{Ag}\end{array}$} & Drawn $5 \%$ & 6400 & & 38,800 & 34 \\
\hline & Rolled 5\% & 2000 & 11,400 & 36,000 & 34 \\
\hline
\end{tabular}

*These data are for short duration tests at $300^{\circ} \mathrm{C}\left(572^{\circ} \mathrm{F}\right)$ 
TABLE 2.2-3

Creep Stress Limits

Data from Reference

6.15

(Long Duration Tests)

\begin{tabular}{|c|c|c|c|c|}
\hline \multirow{2}{*}{ Material } & \multirow{2}{*}{ Condition } & \multicolumn{2}{|c|}{ Temperature } & \multirow{2}{*}{$\begin{array}{c}\text { Creep Stress } \\
\text { Limit } \\
\text { (psi) } \\
0.01 \% \text { per } \\
1000 \mathrm{hrs}\end{array}$} \\
\hline & & ${ }^{\circ} \mathrm{C}$ & ${ }^{\circ} \mathrm{F}$ & \\
\hline \multirow{4}{*}{ OFHC } & Soft & 115 & 240 & 5,125 \\
\hline & $\begin{array}{l}\text { Hard } 84.4 \% \\
\text { Cold Worked }\end{array}$ & 115 & 240 & 26,100 \\
\hline & Soft & 150 & 300 & 3,420 \\
\hline & Hard $84.4 \%$ & 150 & 300 & 8,400 \\
\hline $\begin{array}{l}\mathrm{OFHC}+\mathrm{Ag} \\
0.051 \%\end{array}$ & Soft & 150 & 300 & 10,000 \\
\hline $\mathrm{Cu}+\mathrm{O}_{2}$ & Hard $84.4 \%$ & 100 & 212 & 20,000 \\
\hline
\end{tabular}




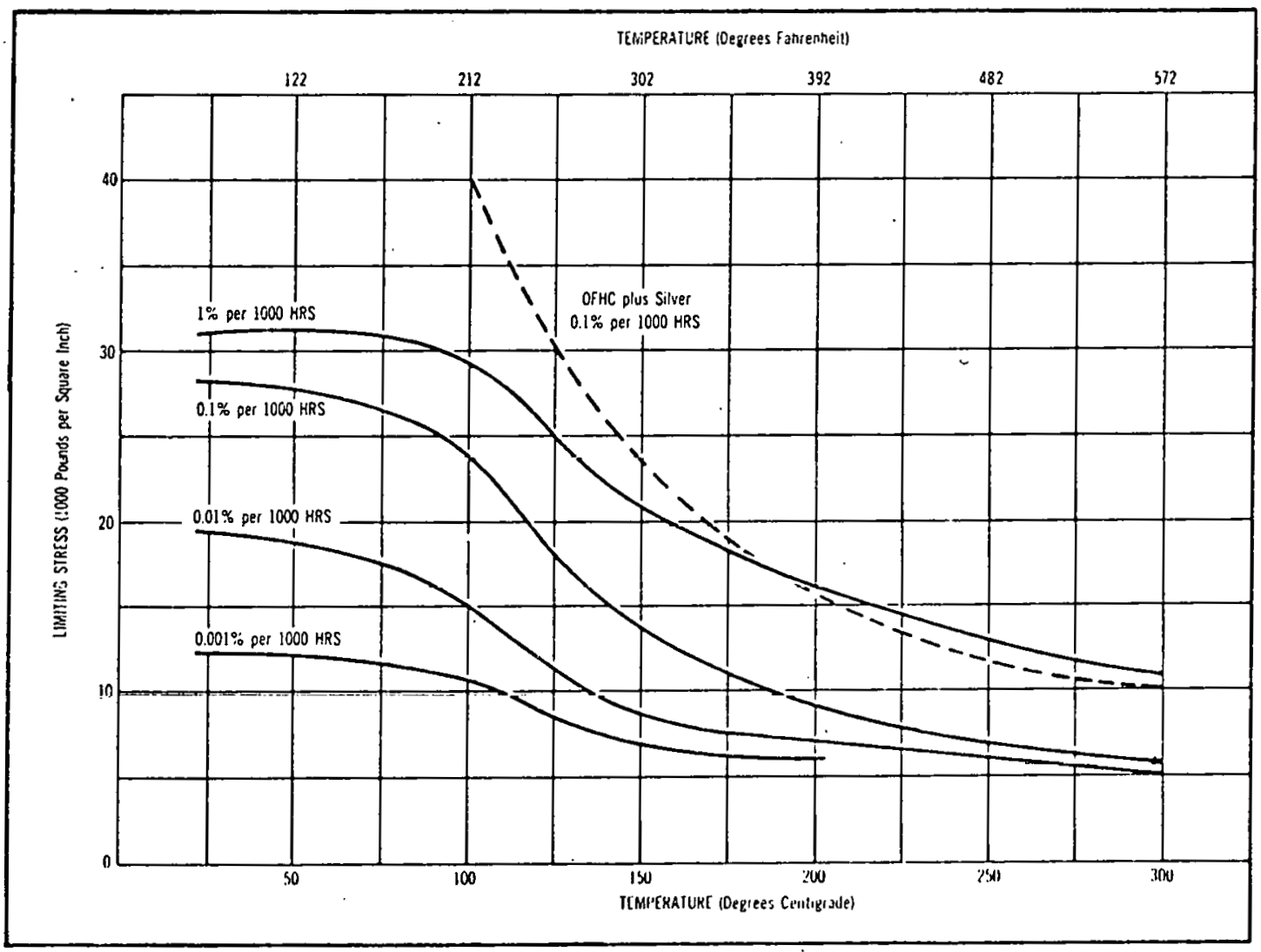

Figure 2.2-1 Temperature-stress-limit curves for OFHC and OFHC plus silver, for various creep rates. 


\subsection{CYCLIC LOAD}

FATIGUE

Figure 2.3-1

Fatigue Strength 


\section{FATIGUE}

The usual fatigue procedures do not show a marked difference between oxygen-bearing and oxygen-free coppers. Some papers report a small superiority for the OFHC brand; the real test, however, is in usage, where a, real difference in service life in favor of the oxygen-free copper has been manifested.

Anderson and Smith (6.17) tested specimens of 0.300-inch minimum diameter, machined from hard-drawn 0.500 -inch-diameter rod, at 3,500 rpm. They report as follows:

OFHC at 300 million cycles: 17,000 psi

Tough pitch at 300 million cycles: 17,000 psi

\section{EFFECT OF ATMOSPHERE}

Gough and Sopwith (6.18) found a higher fatigue strength when the "oxygen-free" copper was tested in moisture-free air or in a partial vacuum than in moist air. The difference was $6 \%$. They conclude, ". . . the effect of atmospheric corrosion-fatigure is due to the catalytic agency of water in the presence of oxygen." Compare with Bleakney (6.19) under "Creep," page 36.

OFHC extruded rod, tested in air, showed a fatigue limit of 4.35 ton/sq. in. $(9,750 \mathrm{psi})$, in $30 \times 10^{6}$ cycles. Diameter of the specimen at the thinnest section was $1 / 2 \mathrm{inch}$.

Bullen, Head, and Wood (6.20). Quoting from the synopsis:

The X-Ray tests show that when a symmetrical cycle in tension-compression is built up slowly, the grains break down on a widespread scale into disoriented elements. But when the same stress cycle is built up faster than a critical rate the disorientation is largely suppressed. The metallographic evidence indicates that there is a corresponding transition from a wide spread to a highly localized distribution of the deformation zones in the grains. 

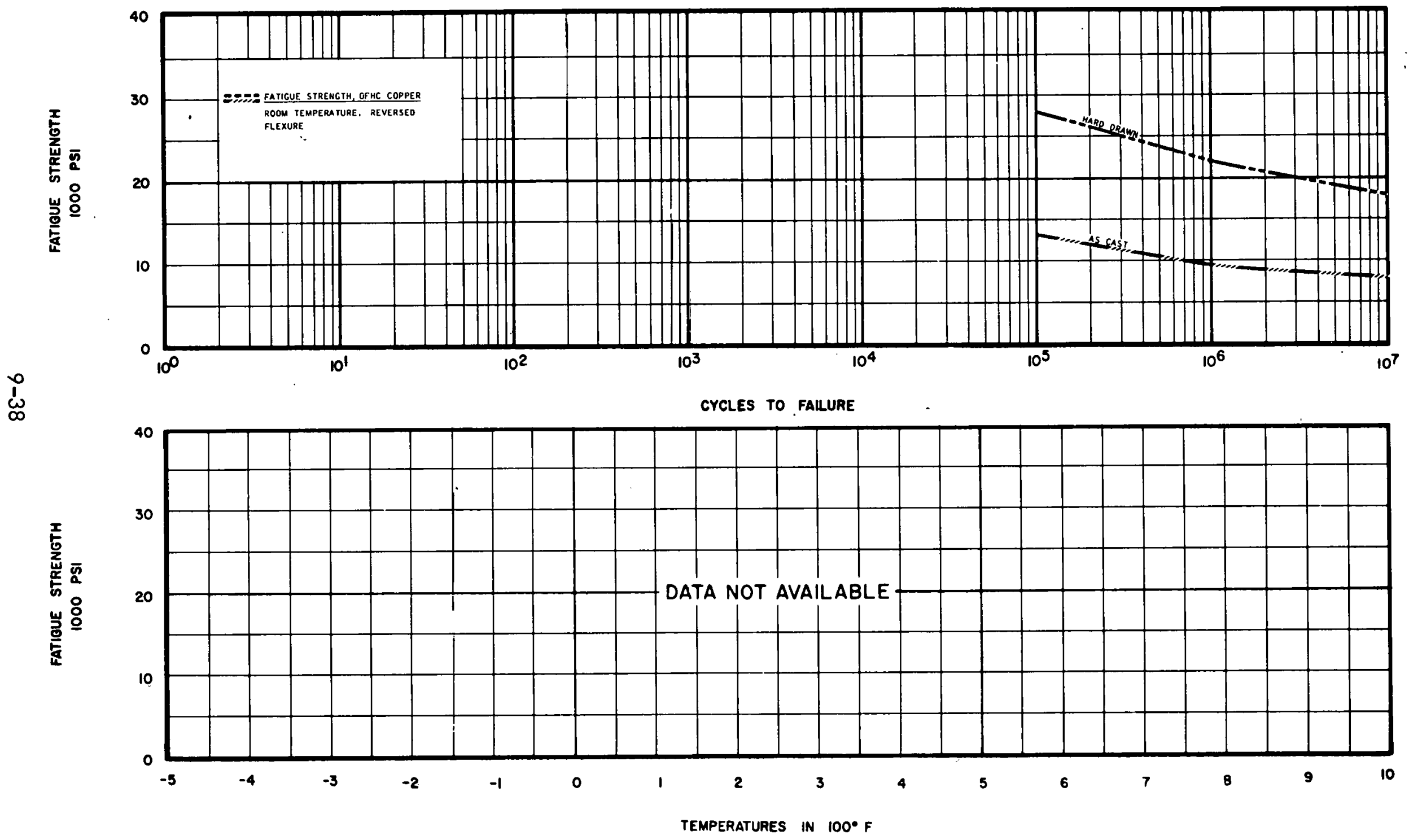

Figure 2.3-1 


\subsection{PHYSICAL PROPERTIES}

3.1 THERMAL PROPERTIES

\section{HEAT CONTENT}

Figure 3.1-1

Heat Contents of Copper

Figure 3.1-2

Specific Heat

\section{THERMAL EXPANSION}

Figure 3.1-3

Thermal Expansion of Copper as Increments of Length at $0^{\circ} \mathrm{C}$.

Figure 3.1-4

Coefficient of Thermal Expansion of Copper

VAPOR PRESSURE

Figure 3.1-5 Vapor Pressure of Copper

Table 3.1-1 Vapor Pressure of OFHC Copper

\section{ELECTRICAL RESISTIVITY}

Figure 3.1-6

How Impurities Offset Conductivity in Pure Copper

Figure 3.1-7

How Cold-Working Affects Conductivity

Figure 3.1-8

Resistivity Versus Temperature 


\section{HEAT CONTENT}

Kelley, in Bulletin 476, U. S. Bureau of Mines, gives a convenient tabulation of heat content of copper, both crystal and liquid. By conversion of the data, three systems of units were derived which were plotted in Figure 3.1-1.

The curves represent the systems as follows:

Degrees Kelvin:

Degrees Centigrade:

Degrees Fahrenheit: cal per gram mole

cal per gram

btu per pound.

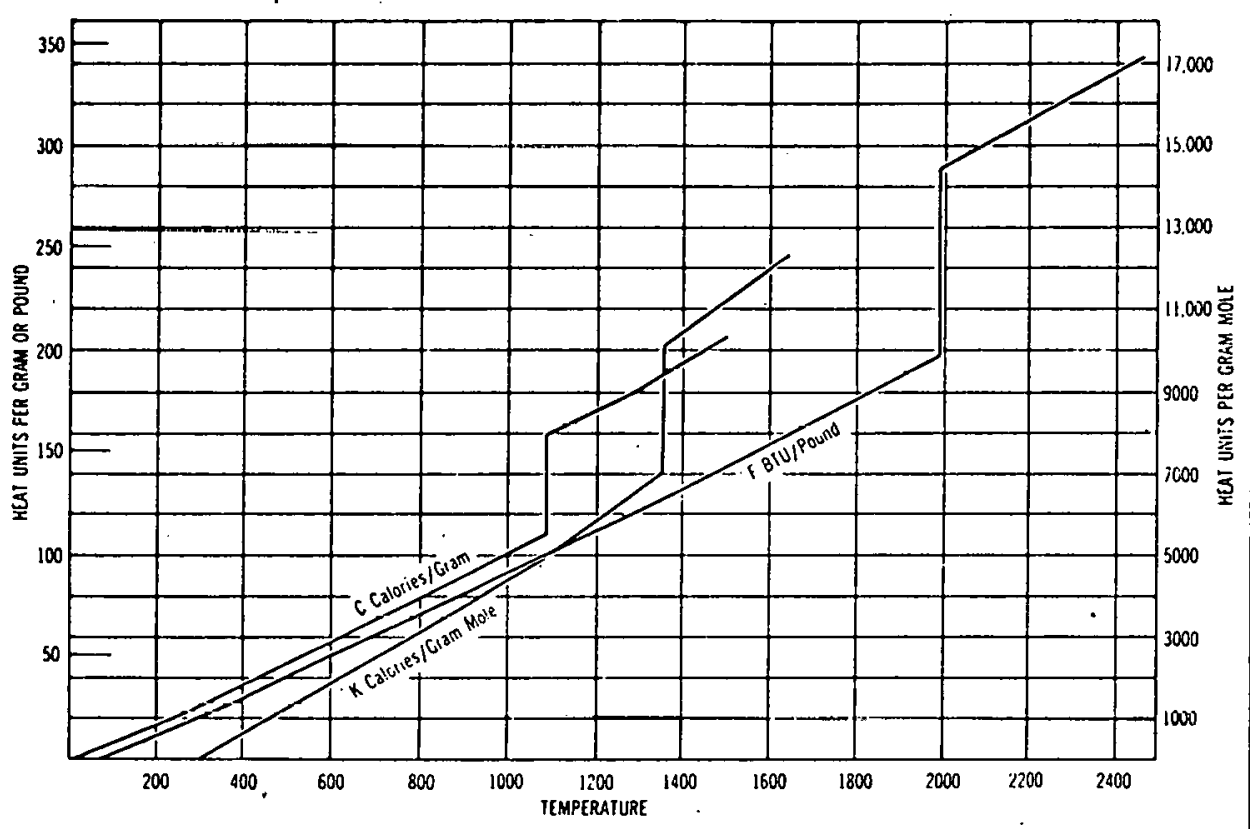

Figure 3.1-1 Heat contents of copper. After data presented by Kelley (Bulletin 476, U. S. Bureau of Mines) 

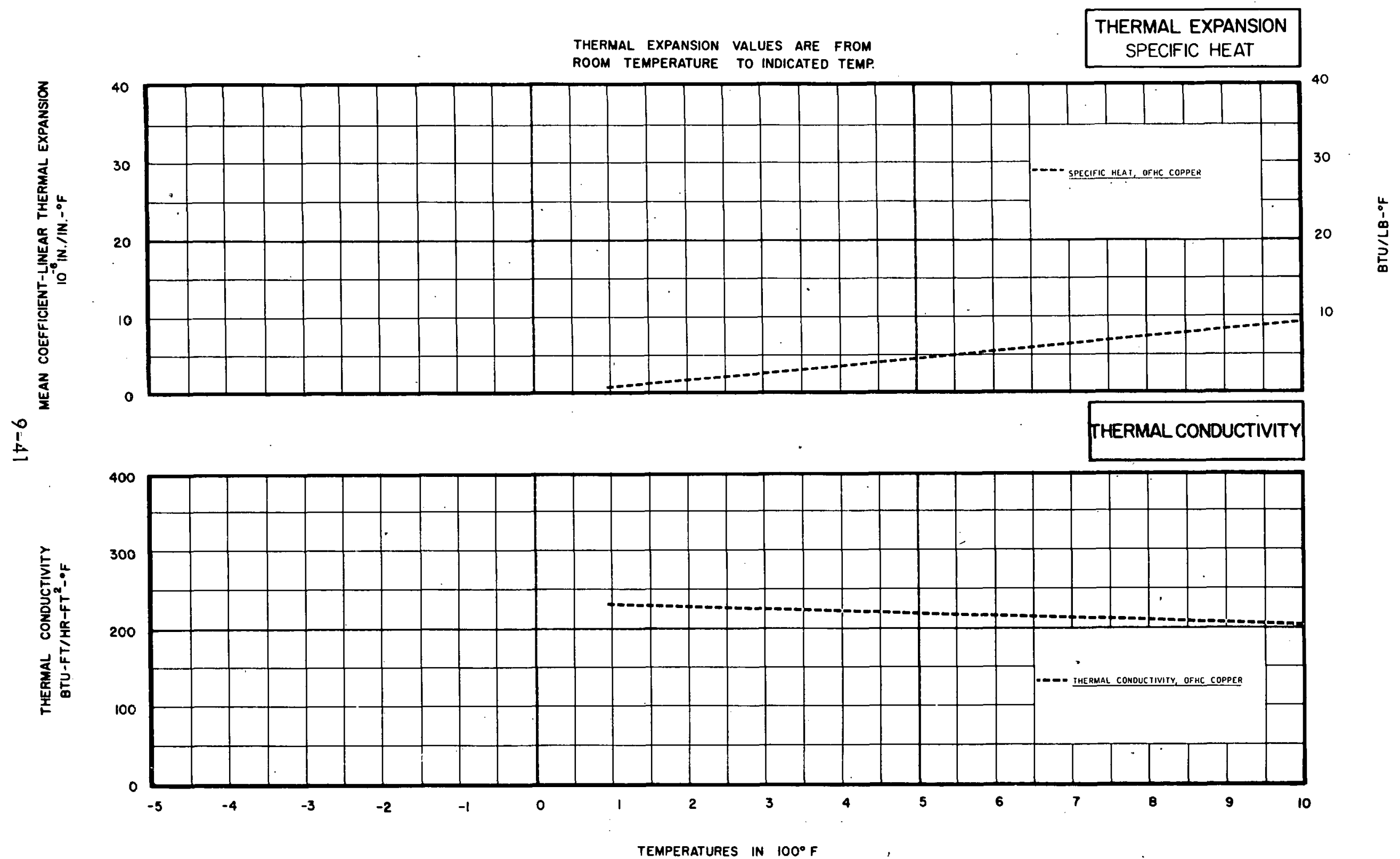

Figure 3.1-2 


\section{THERMAL EXPANSION}

Since it is probable that no essential difference exists between the thermal expansion of OFHC and the "pure" copper cited in the Metals Handbook (6.21), data calculated from the expression for the length increase with temperature have been used to plot the curve in Figure 3.1-3. The equation is as follows:

$$
L_{t}=L_{0}\left[1+\left(16.733 t+0.002626 t^{2}+0.00000091 t^{3}\right) \times 10^{-6}\right]
$$

where $L_{t}=$ length at temperature ${ }^{\circ} \mathrm{G}, L_{0}=$ length at $0^{\circ} \mathrm{C}$.

Richards (6.22) derived the following equation:

$$
L_{t}=L_{0}\left(1+1.67 \times 10^{-5}+3.8 \times 10^{-9} t^{2}+1.5 \times 10^{-12}{ }_{t}^{3}\right)
$$

He states that the expansion of the specimen from $0^{\circ} \mathrm{K}$ to the melting point is $2.78 \%$.

The only paper discovered. in the literature covering the determination of thermal expansion of OFHC copper is that of Rubin, Altman, and Johnson (6.23). The data in that article relate to temperatures from $25^{\circ} \mathrm{K}$ to $295^{\circ} \mathrm{K}\left(-248^{\circ}\right.$ to $\left.+22^{\circ} \mathrm{C}\right)$. Figure $3.1-4$ is adapted from the paper. The curve agrees well with the one in the Metals Handbook (6.21) for pure copper.

For OFHC copper for temperatures above room temperature, Wilkins and Bunn (6.24) give, per degree Centigrade from $25^{\circ} \mathrm{C}$ to $300^{\circ} \mathrm{C}, 0.0000177$, which is the same as they quote for tough-pitch and for phosphorized copper. 
TEMPERATURE (Degrees Fahrenheit)

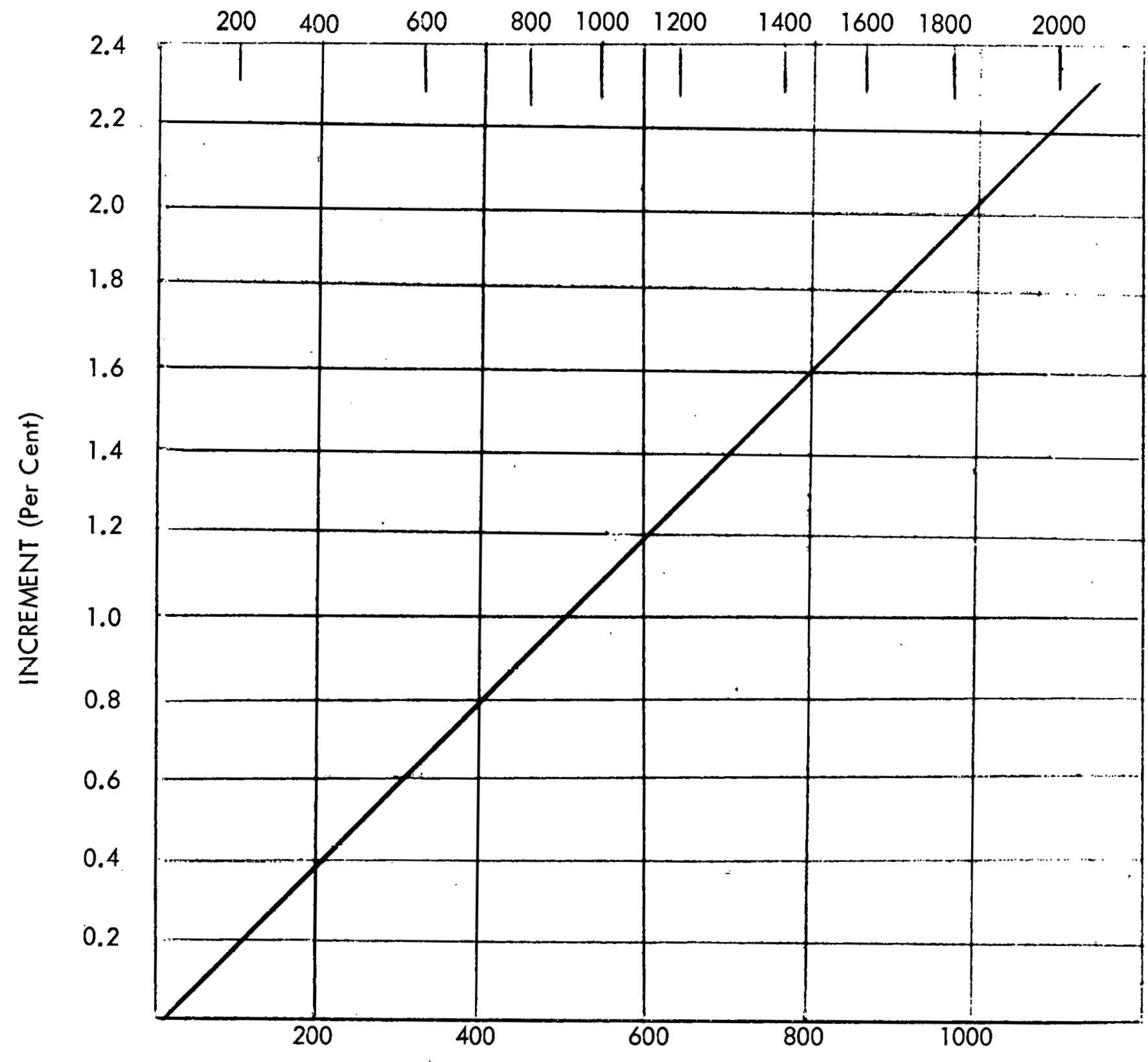

TEMPERATURE (Degrees Centigrade)

Figure 3.1-3 Thermal expansion of copper as increment of length at $0^{\circ} \mathrm{C}$. After equation for length increase with temperature in Metals Handbook (6-21) 


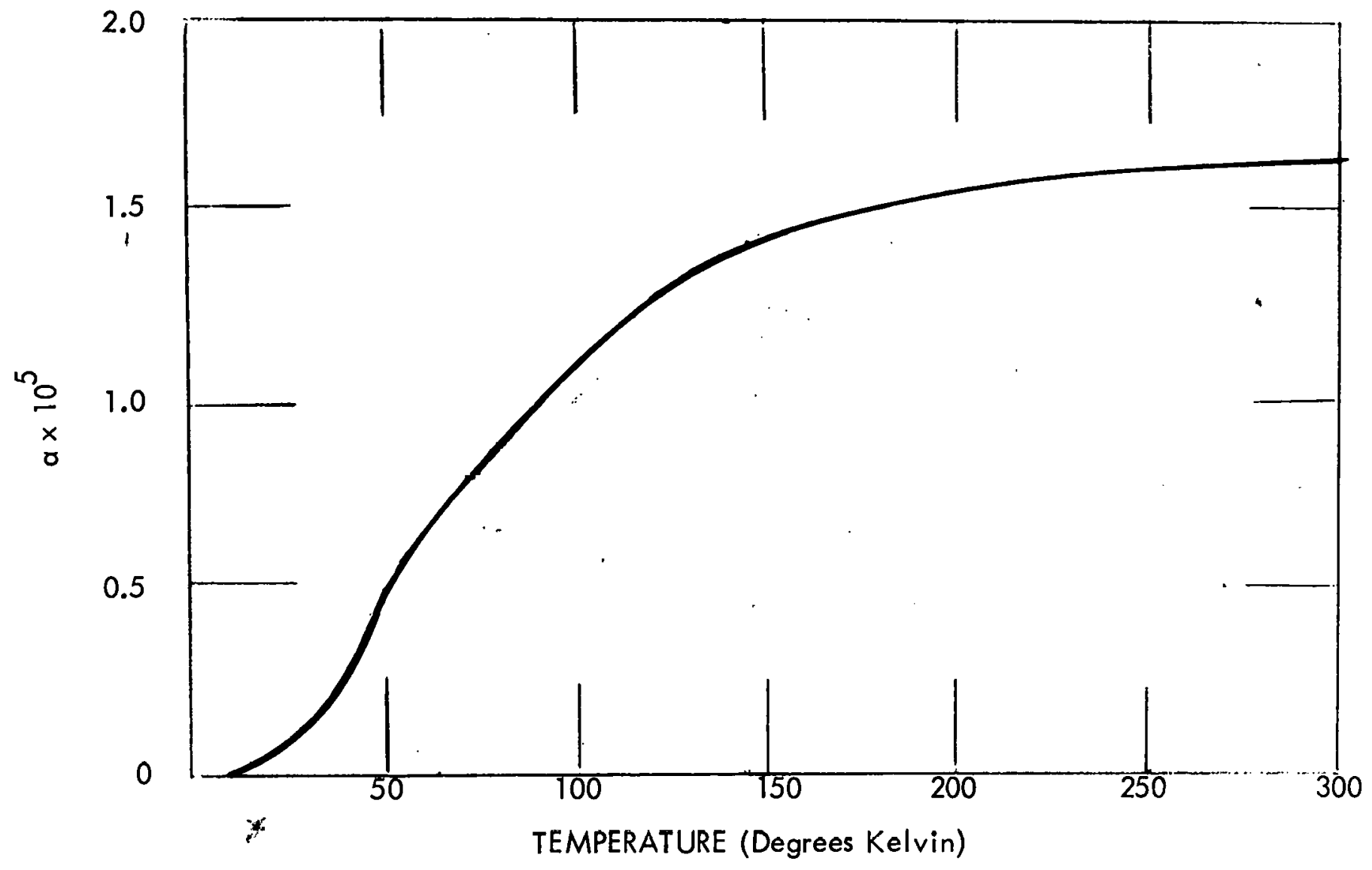

Figure 3.1-4 Coefficient of thermal expansion of copper. 
TABLE 3.1-1

Vapor Pressure of OFHC Copper

\begin{tabular}{|c|c|c|c|c|c|}
\hline & Effective & $\begin{array}{c}\text { Total } \\
\text { Teight } \\
\text { Loss } \\
\text { Time } \\
\text { Temperature } \\
\left({ }^{\circ} \mathrm{K}\right)\end{array}$ & \begin{tabular}{c} 
Effective \\
Area \\
(square \\
\hline 1143 \\
$\left(870^{\circ} \mathrm{C}\right)$
\end{tabular} & $\begin{array}{c}\text { Evaporation } \\
\text { Rate } \\
(\mathrm{g} / \mathrm{cm} /\end{array}$ & $\begin{array}{c}\text { Pressure } \\
\text { (atmospheres } \\
8\end{array}$ \\
\hline $\begin{array}{l}1159 \\
\left(886^{\circ}\right)\end{array}$ & 23,762 & 0.01949 & 33.6386 & 2.438 & 0.233 \\
\hline $\begin{array}{l}1196 \\
\left(923^{\circ} \mathrm{C}\right)\end{array}$ & 6,566 & 0.02387 & 33.7451 & 10.773 & 1.054 \\
\hline $\begin{array}{l}1241 \\
\left(968^{\circ} \mathrm{C}\right)\end{array}$ & 1,740 & 0.02261 & 33.8070 & 38.437 & 3.831 \\
\hline $\begin{array}{l}1292 \\
\left(1020^{\circ} \mathrm{C}\right)\end{array}$ & 1,947 & 0.08872 & 33.8974 & 134.428 & 13.672 \\
\hline
\end{tabular}

Edwards, Johnston, and Ditmars (6.25) used OFHC copper for determining the vapor pressures of copper. The data of Table No. III of their paper are given in Table 3.1-3.

Figure $3.1-5$ is adapted from this paper, somewhat reduced in size.

Hersh (6.26) developed the following equations for the vapor pressure of solid and liquid copper (brand not identified), respectively:

$$
\begin{aligned}
& \text { Solid } \log P_{s}=-\left(1.726 \times 10^{4}\right) / T+9.232 \\
& \text { Liquid } \log P_{1}=-\left(1.682 \times 10^{4}\right) / T+8.907 \\
& 9-45
\end{aligned}
$$




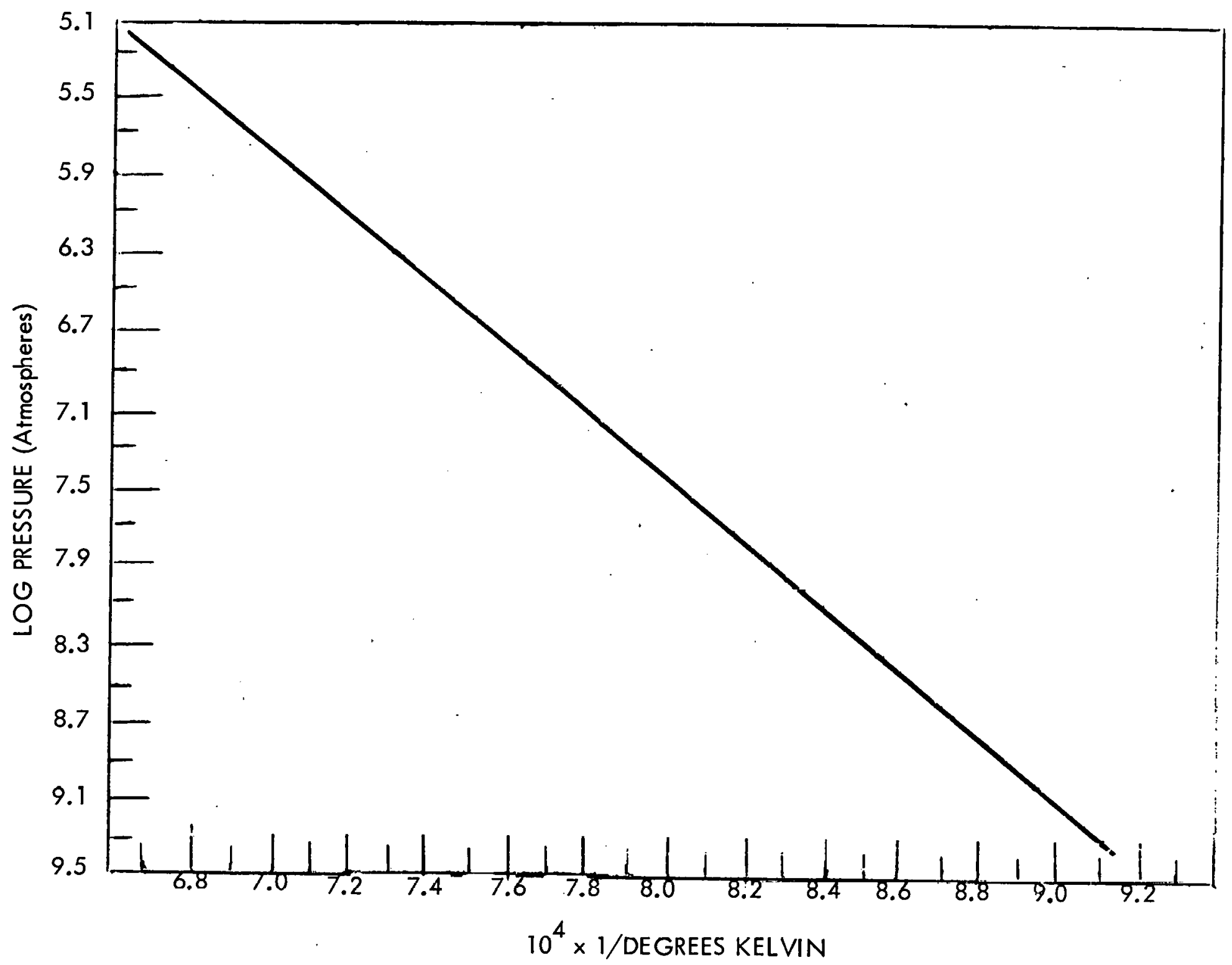

Figure 3.1-5 Vapor pressure of copper. 


\section{ELECTRICAL RESISTIVITY}

All commercial coppers are highly refined but they are all allowed a small quantity of impurities under the applicable specifications. Thus, under ASTM B4 and B5, tough pitch wirebars need have a copper content of $99.90 \%$ copper and a resistivity of 0.15328 ohmgram $/ \mathrm{m}^{2}$, equivalent to $100 \% \mathrm{IACS}$ and $1.72 \mathrm{microhm}-\mathrm{cm}$. The other 1000 parts per million content are actually not defined. The largest single fraction of the remainder will be oxygen. It serves to tie up many of the impurities as oxides and keep them out of solution. Removed from solution, the impurities have little effect on conductivity.

In the case of OFHC copper, the impurities are in solution and marked attention to the effects of various impurities has been made. There have been a number of investigations of the influence of particular elements on the conductivity of copper. All this data has influenced the care with which OFHC copper is produced and the parameters of major concern are spelled out in the certification supplied for the Certified grade of OFHC brand copper.

The effect of impurities on conductivity of OFHC brand copper is magnified by the fact that the absence of oxygen means that each impurity will have an opportunity to go into solution to the limit of its solid solubility and depress the conductivity. Some elements, like iron and phosphorus, for instance, have much greater effects than others.

Besides the impurity content, several factors influence the resistivity or conductivity of OFHC copper and other high conductivity coppers. These include the amount of cold work, and, conversely, the degree of annealing, the operating temperature and the magnetic environment at cryogenic temperatures. 


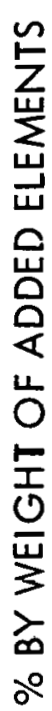

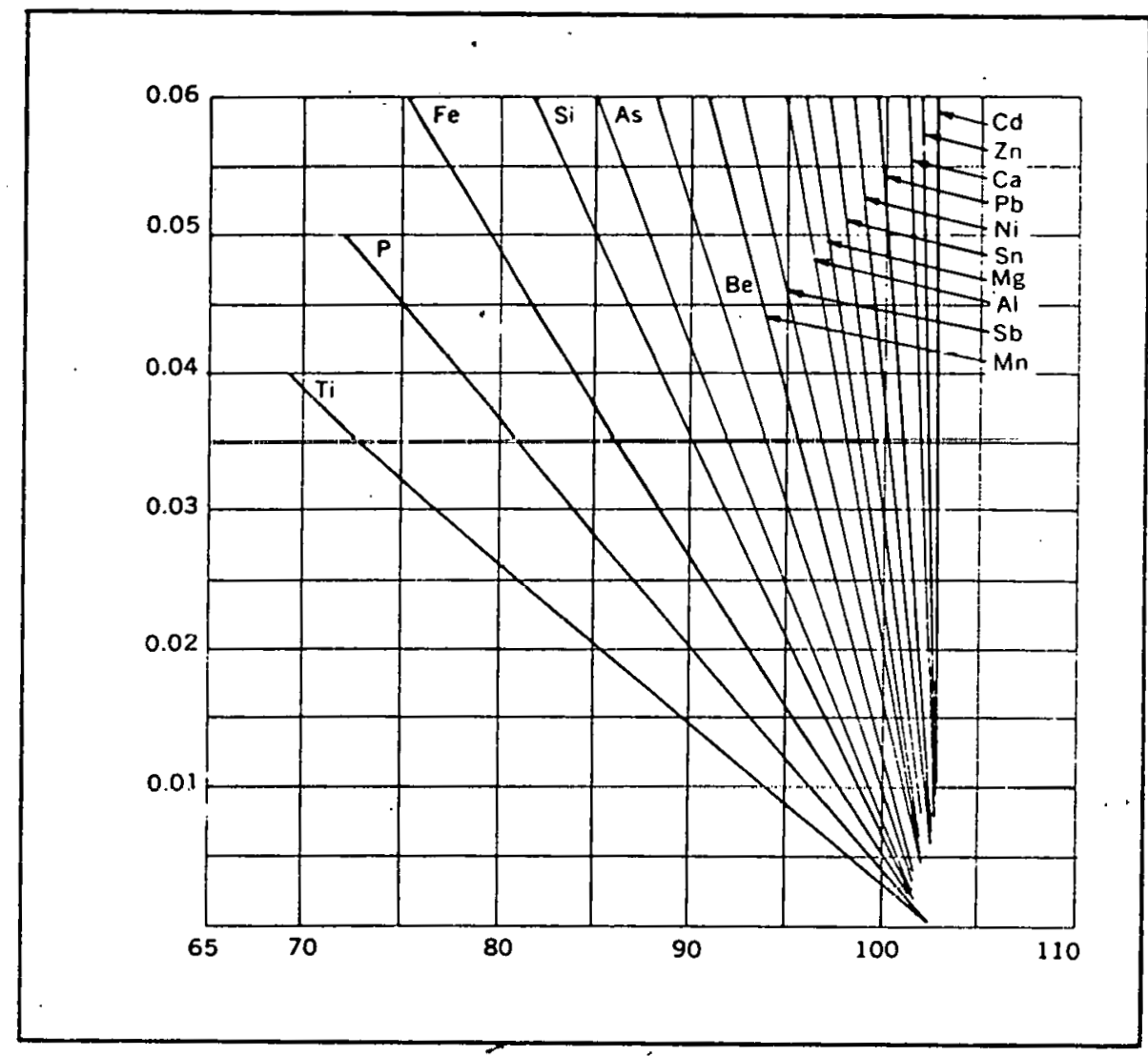

CONDUCTIVITY, \% IACS

Figure 3.1-6 How impurities affect conductivity in pure copper 


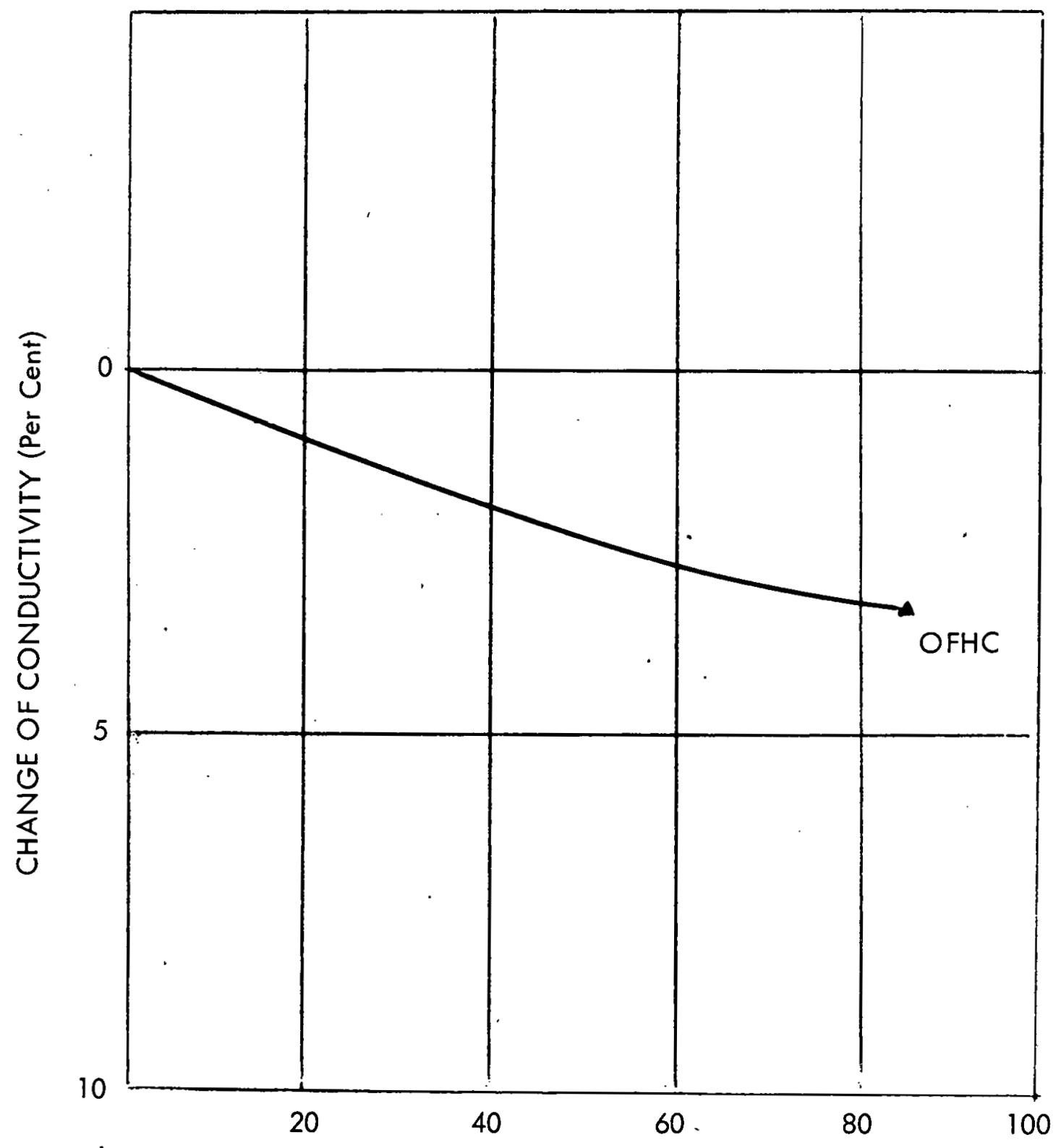

Figure 3.1-7 How cold working affects conductivity 


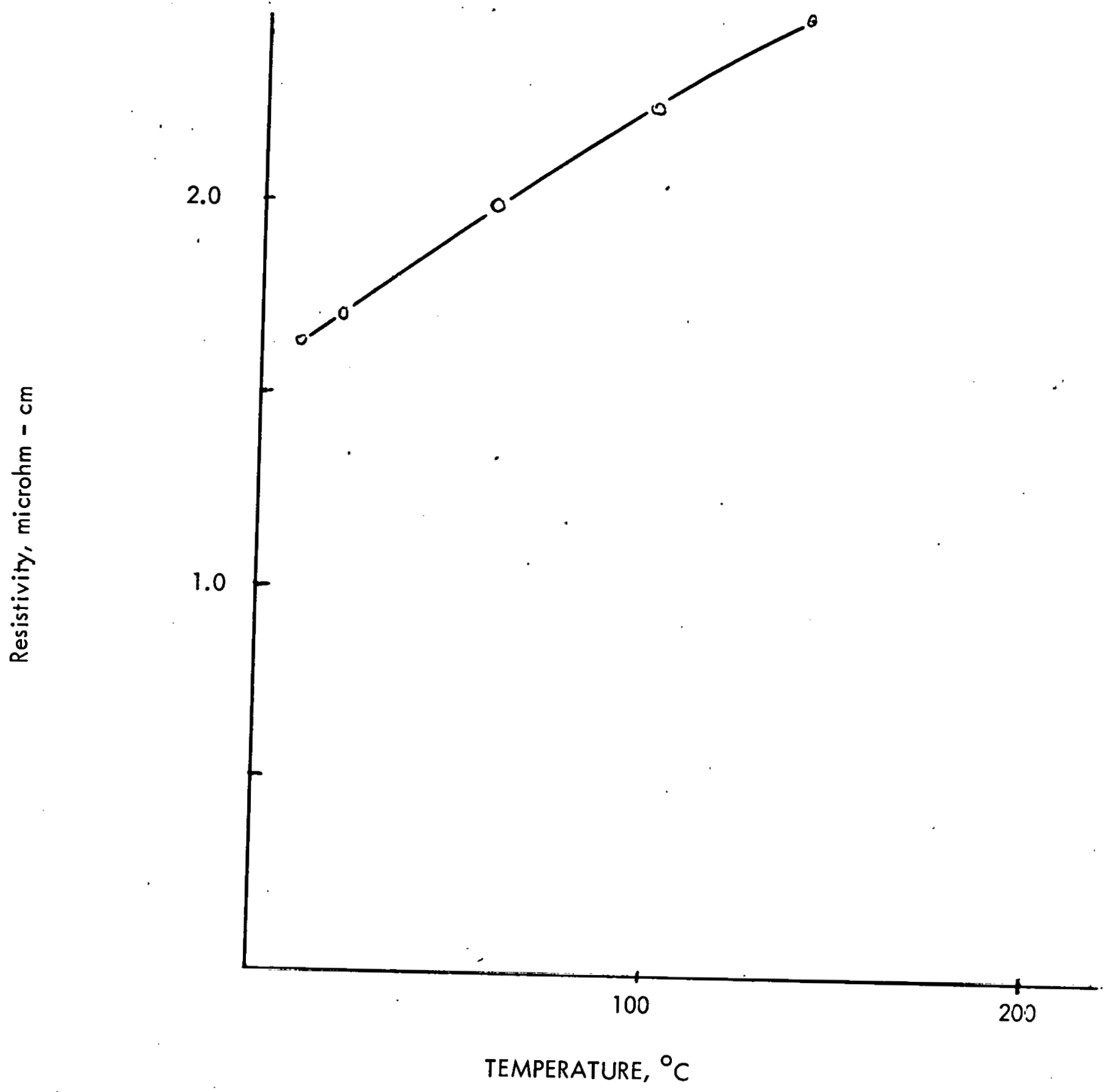

Figure 3.1-8 Resistivity Versus Temperature 


\subsection{ELECTRICAL/MAGNETIC PROPERTIES}

\section{ELECTRICAL CONDUCTIVITY}

Figure 3.2.1 Elevated Temperature Electrical Conductivity of OFHC Copper 


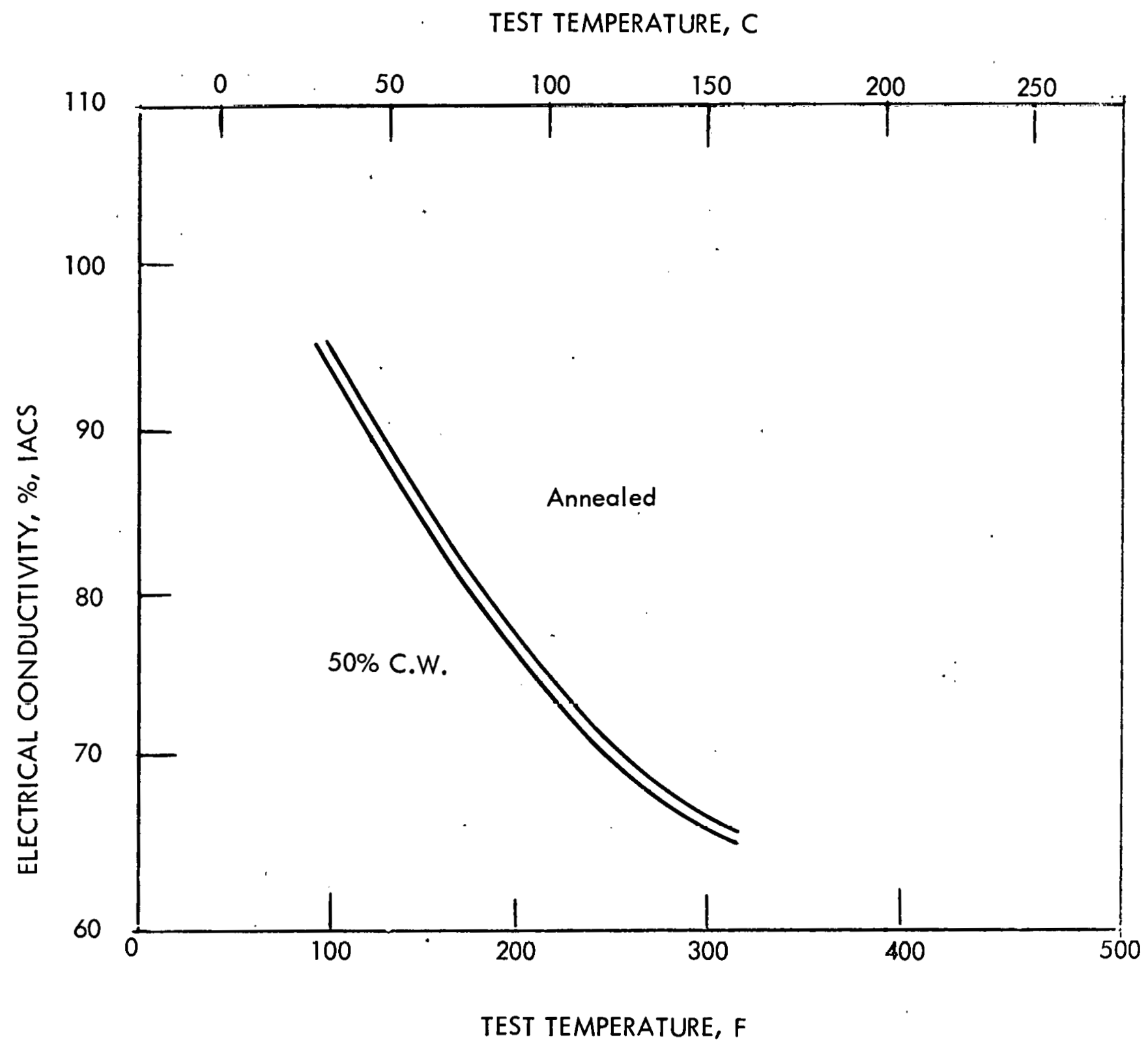

Figure 3.2-1 Elevated temperature electrical conductivity of OFHC Copper (Ref. 6.28) 
4.0 CHEMICAL PROPERTIES

$9-53$ 
5.0 NUCLEAR PROPERTIES

9-54 


\subsection{REFERENCES}

6.1 T. Lyman, editor, "Metals Handbook, "Vol. 1, Properties and Selection of Metals, ASM, 1961.

6.2 OFHC Copper Technical Survey, The American Metal Climax Company, Ltd.

6.3 C. H. Long, et al, "Mechanical Properties of Copper at Elevated Temperatures," Metals and Alloys, Vol. 9, No. 3, 1938.

6.4 Coppers, Materials and Process Material 226, Materials in Design Engineering, Reinhold Publishing Corporation, December, 1964.

6.5 Data from AMCO Laboratory.

6.6 W. O. Jenkins and T. G. Diggs, "Creep of Annealed and Cold-Drawn High Purity Copper," Town. Res. Not. Bur Stand., Vol. 47, 1951, 272.

6.7 N. D. Benson, J. McKeown and D. N. Mends, "The Creep and Softening. Properties of Copper for Alternator Rotor Windings," Town. Inst. Mets., Vol. $80,1951-52,131$.

6.8 W. C. Ellis and E. S. Greiner, "Effect of Prior Strain at Low Temperatures on the Properties of Some Close-Packed Metals at Room Temperature," Town. Mets., June 1952.

6.9 C. S. Smith and R. W. VonWagner, "On the Tensile Properties of Some Copper Alloys," ASTM Proc., Vol. 41, 1941.

6.10 L. R. Jackson, A. M. Hall, and A. D. Schwyse, "Comparative Properties of Several Types of Commercial Coppers, As Cold Worked and as Recrystallized," AIME Tech., Pub. 2274, 1947.

6.11 J. G. Thompson, "The Effect of Cold Rolling on the Indentation."

6.12 C. H. Long, F. B. Dahle and D. A. Roberts, "Mechanical Properties of Copper at Elevated Temperatures," Mets and Alloys, Vol. 9/3, 1938.

6.13 H. J. Miller, "Oxygen in Copper," Metal Treatment, Winter, 1935.

6.14 M. G. Corson, "An Investigation of the Physical Properties of Wire Bars of Electrolytic Copper," AIME Trans. Vol. 128, 1938.

6.15 A. D. Schwape, K. F. Smith, and L. R. Jackson, "Comparative Creep Properties of Several Types of Cómmercial Coppers," AIME Trans. Vol. 185, 1949, p. 409. 


\section{REFERENCES (Continued)}

6.16 H. H. Bleakney, "Ductility of Metals in Creep - Rupture Tests," Canadian Journal Tech., Vol. 30, No. 12, 1952, p. 340.

6.17 A. R. Anderson and C. S. Smith, "Fatigue Tests on Some Copper Alloys," ASTM Proc. Vol. 41, 1941.

6.18 H. J. Lough and D. S. Sapwith, "Some Further Experiments on Atmospheric Action in Fatigue," Town Inst. Mets., Vol. 56, 1935, p. 55.

6.19 H. H. Bleakney, "Ductility of Metals in Creep Rupture Tests," Canadian Journal Tech., Vol. 30, No. 12, 1952, p. 340.

6.20 F. P. Bullen, A. K. Head and W. A. Word, "Structural Changes During Fatigue of Metals," Royal Soc. Proc. A, 1953, pp. 216, 332.

6.21 Metals Handbook, ASM, 1948.

6.22 J. W. Richards, "The Overall Linear Expansion of Three-Face-Centered, Cubic Crystals," Journal Res. Nat. Bur. Stand., Vol. 13, November 1934, RP742.

6.23 T. Rubin, H. A. Altman and H. C. Johnston, "Thermal Expansion of Copper from 15 to $300^{\circ} \mathrm{K}$, Journal Am. Chem. Soc., Vol. 76, 1954, p. 5289.

6.24 R. A. Wilkens and E. S. Bunn, Copper and Copper Base Alloys, McGraw-Hill Book Co., 1933.

6.25 J. W. Edwards, H. C. Johnston, and W. E. Ditmars, "Vapor Pressures of Inorganic Substances," XI, Copper Between 1143 and 1292 K, Amer. Chem. Soc. Journal, Vol. 75, 1953, p. 2467.

6.26 H. N. Hersh, "Vapor Pressure of Copper," Journ. Amer. Chem. Soc., Vol. 75, 1953, p. 1529.

6.27 H. C. Burghoff and A. I. Blank, "Creep Characteristics of Copper and Some Copper Alloys at 300,400 ; and $500^{\circ} \mathrm{F}$, ASTM Proc. Vol. 47, 1947, p. 725.

6.28 N. O. Benson and J. McKeown, "The Creep and Softening Properties of Copper for Altenator Windings," J. Indus. Metals, Vol. 90, 1951, p. 131.

6.29 M. V. Yokelson and M. Balicki, Progressive Work Hardening and Reannealing of Five Brands of High Conductivity of Copper, Copper Wire, and Wire Products, October 1955, p. 1179. 
SECTION 10.0

ELECTRICAL COIL INSULATION 
TABLE OF CONTENTS

1.0

2.0
INSULATION TECHNIQUES

INSULATION MATERIALS (TENTATIVE)

2.1

REINFORCED PLASTIC

2.2

2.3

- eleCtrical tAPE

RESIN 


\subsection{INSULATION TECHNIQUES}

1. 1 TF Coil Insulation System

1. 11 Turn-to-Turn Insulation

Apply two layers of .020" B-stage Scotchply crossplied glass, butt wound, directly over the copper for a total thickness of . 040". The Scotchply will have no pressure sensitive adhesive and the width can be selected to afford the best compromise between optimum winding time and elimination of wrinkling.

1. 12 Coil Insulation

Apply an additional two layers of .020" B-stage Scotchply crossplied glass, butt wound, over the entire coil. The Scotchply.will have no pressure sensitive adhesive and the width can be selected as above in 1.1

1. 13 Pressure Sizing

Size the coil in a suitable pressure mold to provide well controlled dimensions of the final coil. Cure the B-stage resin in the Scotchply while the coil is in the mold, following manufacturer's directions.

1. 14 Additional Ground Insulation

After sizing and curing, wrap the entire coil with 15 layers of '.002" Mylar tape with no adhesive. 


\section{1: 15 Casting Resin}

Before assembly of the coil into the structural support, paint by brush the walls and bottom of the structural support with the epoxy casting resin. Pour uniformly one quart of epoxy resin around the bottom of the structural support and then lower the coil into the support ring.

\section{16 Vacuum Impregnation}

Using the structural form plus a cover as a vacuum vessel, draw a good ( $28^{\prime \prime}$ or better) vacuum on the coil. While maintaining the vacuum, introduce additional resin from at least 6 equally spaced locations around the cover plate. Maintain vacuum for a minimum of 4 hours.

\subsection{Cure}

Remove cover plate. Apply heat to the entire structural form and coil and cure in accordance with resin manufacturer's instructions.

\section{18 Coil Dressing}

Apply a straight edge across the walls of the structural ring forcing a quantity of filled epoxy resin ahead of tool to provide a flat level surface on the coil face. The resin can be either room temperature cure or heat curable.

\section{2 EF and $\mathrm{OH}$ Coil Insulation System}

\subsection{Turn to Turn Insulation}

Apply three layers of . 0032" polyester tape (Scotch No. 1291 or equal) butt wound over each turn of copper to provide a total thickness of . 0096" with the seams of each layer staggered from previous layers. 
1.22 Additional Turn to Turn Insulation

Apply three layers of .011" B-stage Scotchply type 1003, unidirectional, (or equal) butt lapped over each conductor to provide an additional thickness of . 033".

1. 23 Additional Turn to Turn Insulation

Apply an additional three layers as in 1.22.

1. 24 Ground Insulation

Apply three layers of . 020" B-stage Scotchply unidirectional glass, butt wound, over the entire coil to provide an additional thickness of . 060".

1.25 Curing

Provide suitable clamping pressure to the coil and cure the resin tape in accordance with the manufacturer's directions. 
2.0 INSULATION MATERIALS

(Tentative)

$10-4$ 
2.1 REINFORCED PLASTIC

SCOTCHPLY TYPE 1002 AND 1003

$10-5$ 
TABLE OF CONTENTS

1.0 BASIC DATA

$\begin{array}{ll}1.1 & \text { GENERAL } \\ 1.2 & \text { FORMS } \\ 1.3 & \text { SHELF LIFE } \\ 1.4 & \text { RESIN CONTENT } \\ 1.5 & \text { MOLD RELEASE } \\ 1.6 & \text { LINER REMOVAL } \\ 1.7 & \text { MOLDING PROCEDURE } \\ 1.8 & \text { CURE } \\ 1.9 & \text { GENERAL PROPERTIES }\end{array}$

2.0 MECHANICAL PROPERTIES

2.1 MECHANICAL PROPERTIES AT VARIOUS ORIENTATIONS

2.2 MECHANICAL PROPERTIES AT VARIOUS STRESS ANGLES (At $70^{\circ} \mathrm{F}$ )

3.0 PHYSICAL PROPERTIES

3.1 THERMAL PROPERTIES

3.2 ELECTRIC/MAGNETIC PROPERTIES

4.0 CHEMICAL PROPERTIES

5.0 NUCLEAR PROPERTIES

6.0 CURVES

7.0 REFERENCES 


\subsection{BASIC DATA}

\subsection{GENERAL}

"SCOTCHPLY" Reinforced Plastic Type 1002 is a moldable epoxy-glass laminate designed for production cure cycles. It is recommended for general purpose applications requiring high strength over a temperature range of $-60^{\circ} \mathrm{F}$ to $+250^{\circ} \mathrm{F}$. "SCOTCHPLY" is approved under MIL-P-18177C, MIL-P-25421A and the resin used meets MIL-R-9300A.

\subsection{FORMS}

The uncured laminate can be supplied in roll or sheet form. Standard filament orientations are unidirectional, crossply, or isotropic. It is possible to order any special filament orientation the application requires. Listed below are the caliper and sizes in which standard orientation rolls are available:

\begin{tabular}{|l|c|c|}
\hline \multicolumn{1}{|c|}{} & $\begin{array}{c}\text { Average Cured } \\
\text { Thickness }\end{array}$ & $\begin{array}{c}\text { Average Uncured } \\
\text { Thickness }\end{array}$ \\
\hline Undirectional (one ply) & .010 & .011 \\
Crossply (two plies) & .020 & .022 \\
Isotropic (three plies) & .030 & .033 \\
Widths & - & $1 / 4^{\prime \prime}$ to $48^{\prime \prime}$ \\
\hline
\end{tabular}

\subsection{SHELF LIFE}

Six months minimum at $70^{\circ} \mathrm{F}$.

\subsection{RESIN CONTENT}

$36 \%$ by weight 


\subsection{MOLD RELEASE}

The caul plates or matched metal die surfaces must be coated with a suitable mold release for epoxy resin laminates. Some satisfactory products are Minnesota Mining and Manufacturing Company's FC-400, Dow Corning's DC-20 and Ram Chemical Corporation's Garan No. 225.

\subsection{LINER REMOVAL}

Important - The uncured sheets and rolls of "SCOTCHPLY" are protected from foreign matter by a paper liner which must be stripped off prior to molding.

\subsection{MOLDING PROCEDURE}

With the mold at operating temperature, form the part by gradually closing the mold as the laminate becomes thermoplastic. For maximum laminate density, the mold should be brought into immediate contact with the laminate and the press pressure gradually increased until full pressure is reached one to three minutes before gel. For thin laminates the pressure increase may be more rapid than for thick laminates.

\begin{tabular}{|c|c|}
\hline \multicolumn{2}{|c|}{ GEL TIME } \\
\hline TEMPERATURE & GEL TIME \\
\hline $275^{\circ} \mathrm{F}$ & 17 minutes \\
$300^{\circ} \mathrm{F}$ & 9 minutes \\
$325^{\circ} \mathrm{F}$ & $4-1 / 2$ minutes \\
$350^{\circ} \mathrm{F}$ & 2 minutes \\
$375^{\circ} \mathrm{F}$ & 1 minute \\
$400^{\circ} \mathrm{F}$ & 45 seconds \\
\hline
\end{tabular}

\section{MOLD PRESSURE}

25 to 100 psi is recommended. Type 1002 may be vacuum bag molded at 10 to 15 psi with a 10 to $20 \%$ reduction in mechanical properties. 


\subsection{CURE}

Two recommended cure cycles are listed in the table below for molding high strength laminates of "SCOTCHPLY" Type 1002. Cure Cycle No. 1 and Cure Cycle No. 2 both produce room temperature mechanical properties as listed in the "Mechanical Properties" table. With the elimination of the oven post-cure in Cure Cycle No. 1 room temperature mechanical properties will be $85 \%$ of those values listed. For maximum high temperature properties $300^{\circ} \mathrm{F}$ post cure should be used with Cycles No. 1 and No. 2.

TABLE

\begin{tabular}{|c|c|c|c|}
\hline \multirow{3}{*}{ Thickness } & \multicolumn{2}{|c|}{$\begin{array}{c}\text { CURE CYCLE NO. 1 } \\
\text { (press and post cure) }\end{array}$} & $\begin{array}{c}\text { CURE CYCLE NO. 2 } \\
\text { (press only) }\end{array}$ \\
\cline { 2 - 4 } $1 / 8$ & $\begin{array}{c}\text { Press Time } \\
\text { at } 330^{\circ} \mathrm{F}\end{array}$ & $\begin{array}{c}\text { Oven Post Cure } \\
\text { at } 280^{\circ} \mathrm{F}\end{array}$ & $\begin{array}{c}\text { Press Time } \\
\text { at } 330^{\circ} \mathrm{F}\end{array}$ \\
\cline { 2 - 4 } $1 / 4$ & 5 minutes & 16 hours & 35 minutes \\
$1 / 2$ & 10 minutes & 16 hours & 40 minutes \\
1 & 15 minutes & 16 hours & 45 minutes \\
& 25 minutes & 16 hours & 55 minutes \\
\hline
\end{tabular}

\subsection{GENERAL PROPERTIES}

Flammability (inches min.). .... 0.10

Barcol Hardness ......... 70

Rockwell Hardness (M) Scale) .....100-108

Specific Gravity . . . . . . . . . 1.8

Molded Thickness (one ply) . . . .010"

Wet Strength Retention

(2 Hr. Boil).......... 86\% 


\subsection{MECHANICAL PROPERTIES}

\subsection{MECHANICAL PROPERTIES AT VARIOUS ORIENTATIONS}

\begin{tabular}{|c|c|c|c|c|c|c|}
\hline \multirow[b]{2}{*}{ Orientation } & \multirow[b]{2}{*}{ Mechanical Property } & \multicolumn{5}{|c|}{ Temperature ${ }^{\circ} \mathrm{F}$} \\
\hline & & -60 & 70 & 160 & 250 & 300 \\
\hline \multirow{5}{*}{ 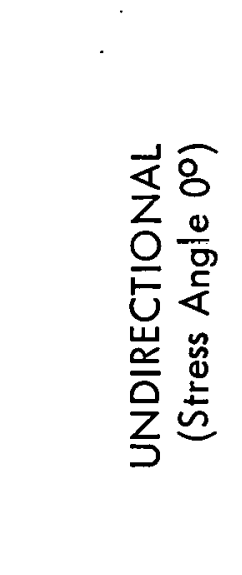 } & $\begin{array}{l}\text { Flexural Strength - } \\
\text { PSI } \times 10^{3}\end{array}$ & 192 & 165 & 128 & 80 & 30 \\
\hline & $\begin{array}{l}\text { Modulus in Flexure - } \\
\text { PSI } \times 10^{6}\end{array}$ & 5.4 & 5.3 & 5.0 & 4.2 & 1.0 \\
\hline & $\begin{array}{l}\text { Tensile Strength - } \\
\text { PSI } \times 10^{3}\end{array}$ & 172 & 160 & 139 & 105 & 50 \\
\hline & $\begin{array}{l}\text { Modulus in Tension - } \\
\text { PSI } \times 10^{6}\end{array}$ & & 5.7 & & & \\
\hline & $\begin{array}{l}\text { Compression Strength, Edge - } \\
\text { PSI } \times 10^{3}\end{array}$ & 115 & 90 & 70 & 45 & 10 \\
\hline
\end{tabular}

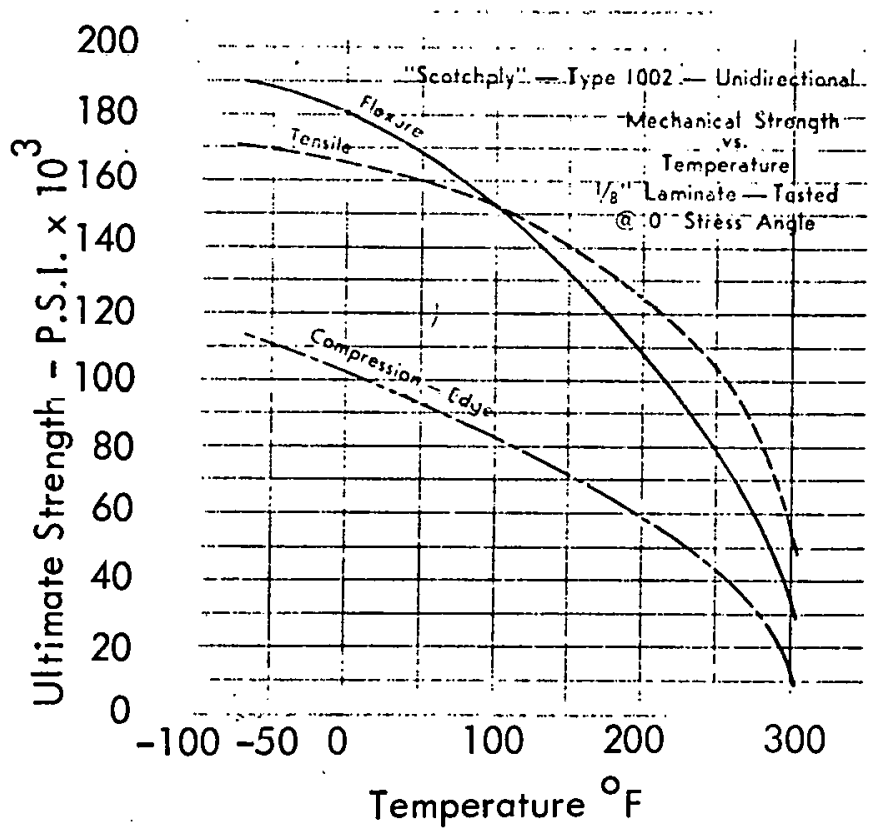




\begin{tabular}{|c|c|c|c|c|c|c|}
\hline \multirow[b]{2}{*}{ Orientation } & \multirow[b]{2}{*}{ Mechanical Property } & \multicolumn{5}{|c|}{ Temperature ${ }^{\circ} \mathrm{F}$} \\
\hline & & -60 & 70 & 160 & 250 & 300 \\
\hline \multirow{5}{*}{ 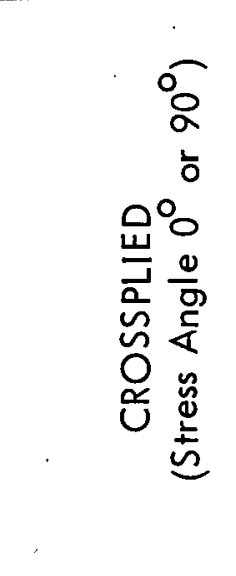 } & $\begin{array}{l}\text { Flexural Strength - } \\
\text { PSI } \times 10^{3}\end{array}$ & 137 & 120 & 104 & 75 & 25 \\
\hline & $\begin{array}{l}\text { Modulus in Flexure - } \\
\text { PSI } \times 10^{6}\end{array}$ & 3.6 & 3.5 & 3.3 & 2.8 & 0.8 \\
\hline & $\begin{array}{l}\text { Tensile Strength - } \\
\text { PSI } \times 10^{3} \\
\end{array}$ & 92 & 75 & 62 & 50 & 40 \\
\hline & $\begin{array}{c}\text { Modulus in Tension - } \\
\text { PSI } \times 10^{6}\end{array}$ & & 3.7 & 3.0 & 2.6 . & \\
\hline & $\begin{array}{l}\text { Compression Strength, Edge - } \\
\text { PSI } \times 10^{3}\end{array}$ & 82 & .75 & 66 & 45 . & 20 \\
\hline
\end{tabular}

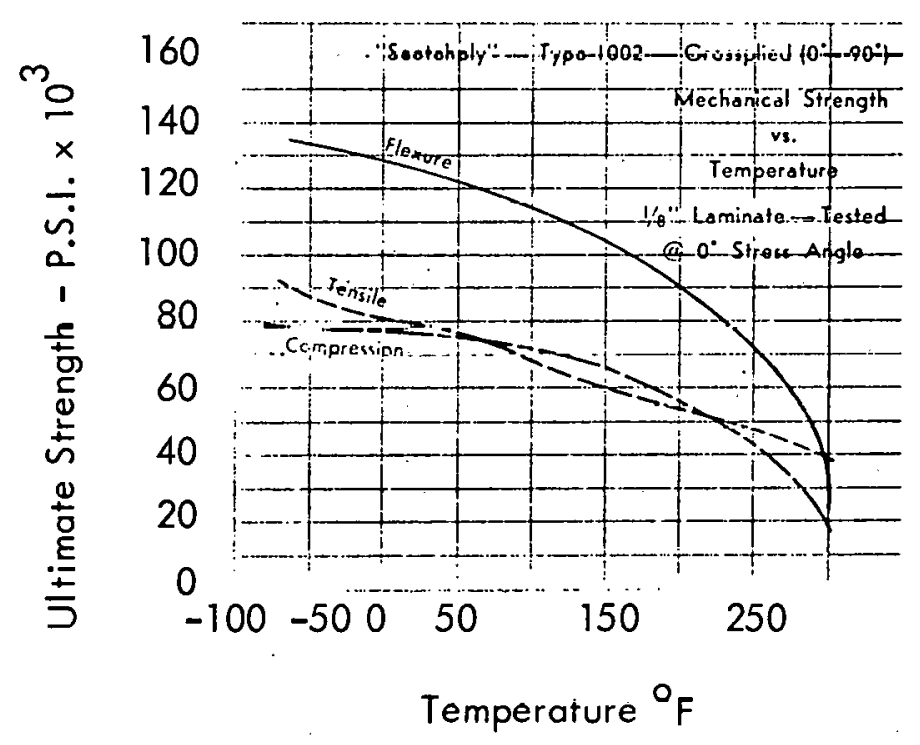




\begin{tabular}{|c|c|c|c|c|c|c|}
\hline \multirow[b]{2}{*}{ Orientation } & \multirow[b]{2}{*}{ - Mechanical Property } & \multicolumn{5}{|c|}{ Temperature ${ }^{\circ} \mathrm{F}$} \\
\hline & & -60 & 70 & 160 & 250 & 300 \\
\hline \multirow{5}{*}{ 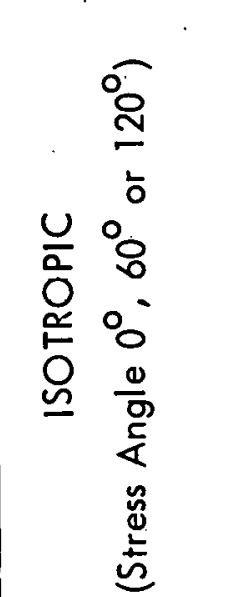 } & $\begin{array}{l}\text { Flexural Strength - } \\
\text { PSI } \times 10^{3}\end{array}$ & 104 & 80 & 70 & 55 & 16 \\
\hline & $\begin{array}{l}\text { Modulus in Flexure - } \\
\text { PSI } \times 10^{6}\end{array}$ & 2.8 & 3.0 & 2.7 & 1.8 & 0.8 \\
\hline & $\begin{array}{c}\text { Tensile Strength - } \\
\text { PSI } \times 10^{3}\end{array}$ & 69 & 57 & 51 & 48 & 36 \\
\hline & $\begin{array}{c}\text { Modulus in Tension - } \\
\text { PSI } \times 10^{6}\end{array}$ & & 2.6 & 2.3 & 1.9 & \\
\hline & $\begin{array}{l}\text { Compression Strength, Edge - } \\
\text { PSI } \times 10^{3}\end{array}$ & 70 & 60 & 52 & 38 & 18 \\
\hline
\end{tabular}

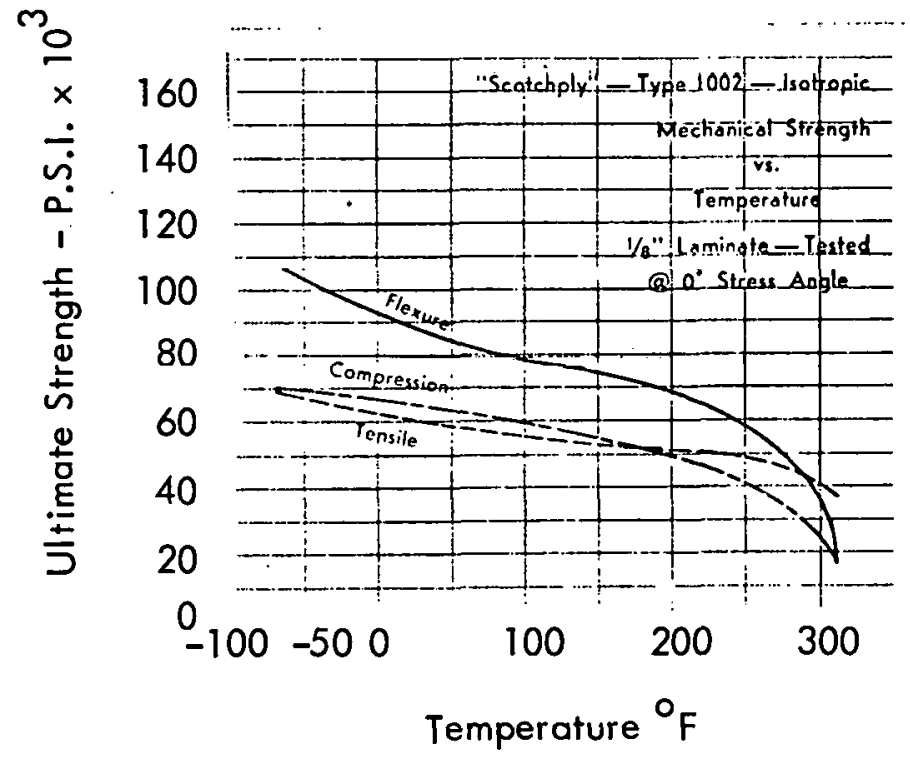




\begin{tabular}{|c|c|c|c|}
\hline \multirow[b]{2}{*}{ UNDIRECTIONAL } & \multicolumn{3}{|c|}{ STRESS ANGLE } \\
\hline & $0^{\circ}$ & $45^{\circ}$ & $90^{\circ}$ \\
\hline $\begin{array}{l}\text { Flexure Strength - } \\
\text { PSI } \times 10^{3} \\
\end{array}$ & 165 & 20 & 10 \\
\hline $\begin{array}{l}\text { Modulus in Flexure - } \\
\qquad \mathrm{PSI} \times 10^{6}\end{array}$ & 5.3 & 2.0 & 1.6 \\
\hline $\begin{array}{l}\text { Tensile Strength - } \\
\text { PSI } \times 10^{3} \\
\end{array}$ & 160 & 3.6 & 2.9 \\
\hline $\begin{array}{c}\text { Modulus in Tensile - } \\
\text { PSI } \times 10^{6}\end{array}$ & 5.7 & 1.4 & 1.4 \\
\hline $\begin{array}{l}\text { Compression Strength, Edge - } \\
\text { PSI } \times 10^{3}\end{array}$ & 90 & 25 & 20 \\
\hline $\begin{array}{l}\text { Izod Impact (Edgewise) } \\
\qquad(\text { Ft. Lbs./Inch Notch) }\end{array}$ & 60.8 & 6.8 & 0.9 \\
\hline $\begin{array}{l}\text { *Interlaminar Single Shear Strength - } \\
\qquad \text { PSI } \times 10^{3}\end{array}$ & 4.3 & & \\
\hline \multirow[b]{2}{*}{ CROSSPLIED } & \multicolumn{3}{|c|}{ SIRESS ANGLE } \\
\hline & $0^{\circ}$ & $45^{\circ}$ & $90^{\circ}$ \\
\hline $\begin{array}{l}\text { Flexure Strength - } \\
\mathrm{PSI} \times 10^{3} \\
\end{array}$ & 120 & 50 & 120 \\
\hline $\begin{array}{l}\text { Modulus in Flexure - } \\
\qquad \text { PSI } \times 10^{6}\end{array}$ & 3.5 & 2.0 & 3.5 \\
\hline $\begin{array}{l}\text { Tensile Strength - } \\
\text { PSI } \times 10^{3} \\
\end{array}$ & 75 & 22 & 75 \\
\hline $\begin{array}{l}\text { Modulus in Tensile - } \\
\text { PSI } \times 10^{6} \\
\end{array}$ & 3.7 & 1.6 & 3.7 \\
\hline $\begin{array}{l}\text { Compression Strength, Edge - } \\
\text { PSI } \times 10^{3}\end{array}$ & 75 & 23 & 75 \\
\hline $\begin{array}{l}\text { Izod Impact (Edgewise) } \\
\qquad \text { (Ft. Lbs./Inch Notch) }\end{array}$ & 35.2 & 59.2 & 35.2 \\
\hline $\begin{array}{l}\text { *Interlaminar Single Shear Strength - } \\
\text { PSI } \times 10^{3}\end{array}$ & 4.1 & & \\
\hline
\end{tabular}

*3M test procedure

Flat compression on unit and ply $\sim 15,000-20,000$ 


\begin{tabular}{|c|r|r|r|}
\hline & \multicolumn{3}{|c|}{ STRESS ANGLE } \\
\cline { 2 - 4 } ISOTROPIC & $0^{\circ}$ & $45^{\circ}$ & $90^{\circ}$ \\
\hline $\begin{array}{c}\text { Flexure Strength - } \\
\text { PSI } \times 10^{9}\end{array}$ & 80 & 73 & 79 \\
\hline $\begin{array}{c}\text { Modulus in Flexure - } \\
\text { PSI } \times 10^{6}\end{array}$ & 3.0 & 2.8 & 2.7 \\
\hline $\begin{array}{c}\text { Tensile Strength - } \\
\text { PSI } \times 10^{3}\end{array}$ & 57 & 43 & 43 \\
\hline $\begin{array}{c}\text { Modulus in Tensile - } \\
\text { PSI } \times 10^{6}\end{array}$ & 2.6 & 2.9 & 2.9 \\
\hline $\begin{array}{c}\text { Compression Strength, Edge - } \\
\text { PSI } \times 10^{3}\end{array}$ & 60 & 51 & 51 \\
\hline $\begin{array}{c}\text { Izod Impact (Edgewise) - } \\
\text { (Ft. Lbs./Inch Notch) }\end{array}$ & 40.8 & 42.3 & 38.6 \\
\hline $\begin{array}{c}\text { *Interlaminar Single Shear Strength on } \\
\text { PSI } \times 10^{3}\end{array}$ & 3.7 & & \\
\hline
\end{tabular}

*3M test procedure.

Flat compression on unit and ply $\sim 15,000-20,000$ psi. 


\subsection{PHYSICAL PROPERTIES}

3.1 - THERMAL PROPERTIES

THERMAL PROPERTIES

COEFFICIENT OF LINEAR EXPANSION

Method - ASTM D696-42T

Tested over Temperature Range of $-30^{\circ} \mathrm{F}$ to $+200^{\circ} \mathrm{F}$

\begin{tabular}{|l|l|l|}
\hline $\begin{array}{c}\text { FIBER } \\
\text { ORIENTATION }\end{array}$ & DIRECTION OF MEASUREMENT & $\begin{array}{l}\text { COEFFICIENT OF LINEAR } \\
\text { EXPANION PER OF }\end{array}$ \\
\hline Isotropic & Parallel to one array of filaments & $8.4 \times 10^{-6}$ \\
Crossplied & Parallel to lengthwise filaments & $7.1 \times 10^{-6}$ \\
Crossplied & Parallel to crosswise filaments & $7.1 \times 10^{-6}$ \\
Unidirectional & Parallel to all filaments & $4.8 \times 10^{-6}$ \\
Unidirectional & Perpendicular to all filaments & $12.3 \times 10^{-6}$ \\
\hline
\end{tabular}

THERMAL CONDUCTIVITY (k)

$(k)=\frac{\text { B.t.u. of heat transmitted through } 1 \text { inch thickness }}{\left({ }^{\circ} \mathrm{F}\right) \quad(\text { sq. ft. })}$

Tests conducted on a 1 inch thick isotropic laminate

\begin{tabular}{|c|c|}
\hline MEAN TEMPERATURE & THERMAL CONDUCTIVITY $(\mathrm{k})$ \\
\hline $36^{\circ} \mathrm{F}$ & 2.33 \\
$45^{\circ} \mathrm{F}$ & 2.32 \\
$125^{\circ} \mathrm{F}$ & 2.37 \\
$186^{\circ} \mathrm{F}$ & 2.40 \\
\hline
\end{tabular}

Specific Heat $=0.21$ Cal/GM-DEG. C 


\subsection{ELECTRIC/MAGNETIC PROPERTIES}

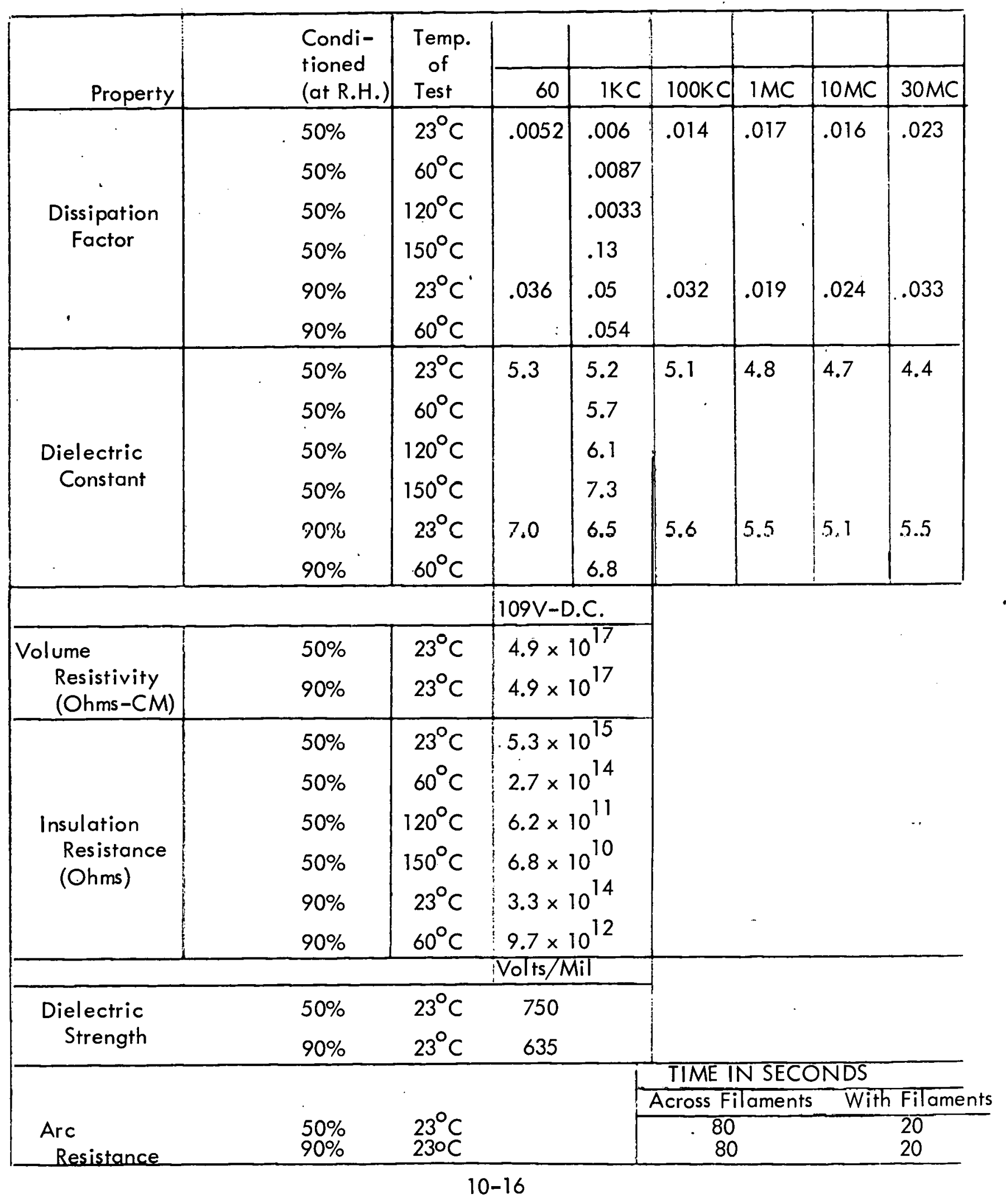




\subsection{CHEMICAL PROPERTIES}

\begin{tabular}{|c|c|c|c|}
\hline \multicolumn{4}{|c|}{$\begin{array}{c}\text { CROSSPLY }\left(0^{\circ}-90^{\circ}\right) \text { FIBER ORIENTATION } \\
\text { Test Method ASTM D543-T } 43 \\
7 \text { day immersion at } 70^{\circ} \mathrm{F}\end{array}$} \\
\hline CHEMICAL & $\begin{array}{l}\text { PER CENT } \\
\text { CHANGE IN } \\
\text { WEIGHT }\end{array}$ & $\begin{array}{l}\text { PER CENT } \\
\text { CHANGE IN } \\
\text { THICKNESS }\end{array}$ & $\begin{array}{l}\text { FLEXURAL } \\
\text { STRENGTH } \\
\text { PSI } \times 10^{3}\end{array}$ \\
\hline Heptane & +.02 & +.02 & 110 \\
\hline Isopropyl Alcohol & +.16 & +.23 & 104 \\
\hline Ethylene Glycol & +.06 & +.02 & 109 \\
\hline Jet Engine Oil & +.08 & -.14 & 111 \\
\hline Jet Fuel (JP-4) & +.07 & +.11 & 111 \\
\hline Hydraul ic Aircraft Oil & +.07 & -.05 & 114 \\
\hline Sulfuric Acid $3 \%$ & +.06 & +.09 & 100 \\
\hline Sulfuric Acid $30 \%$ & +.07 & +.24 & 101 \\
\hline Sodium Hydroxide $1 \%$ & +.19 & +.04 & 95 \\
\hline Sodlum Hydroxide $10 \%$ & +.20 & +.12 & 94 \\
\hline Hydrogen Peróxide $3 \%$ & +.21 & +.10 & 110 \\
\hline Distilled Water & +.17 & +.09 & 109 \\
\hline
\end{tabular}




\subsection{NUCLEAR PROPERTIES}

$10-18$ 


\subsection{CURVES}

. NOTE: CREEP CURVES FROM LAZAN U OF M FROCEDURE.
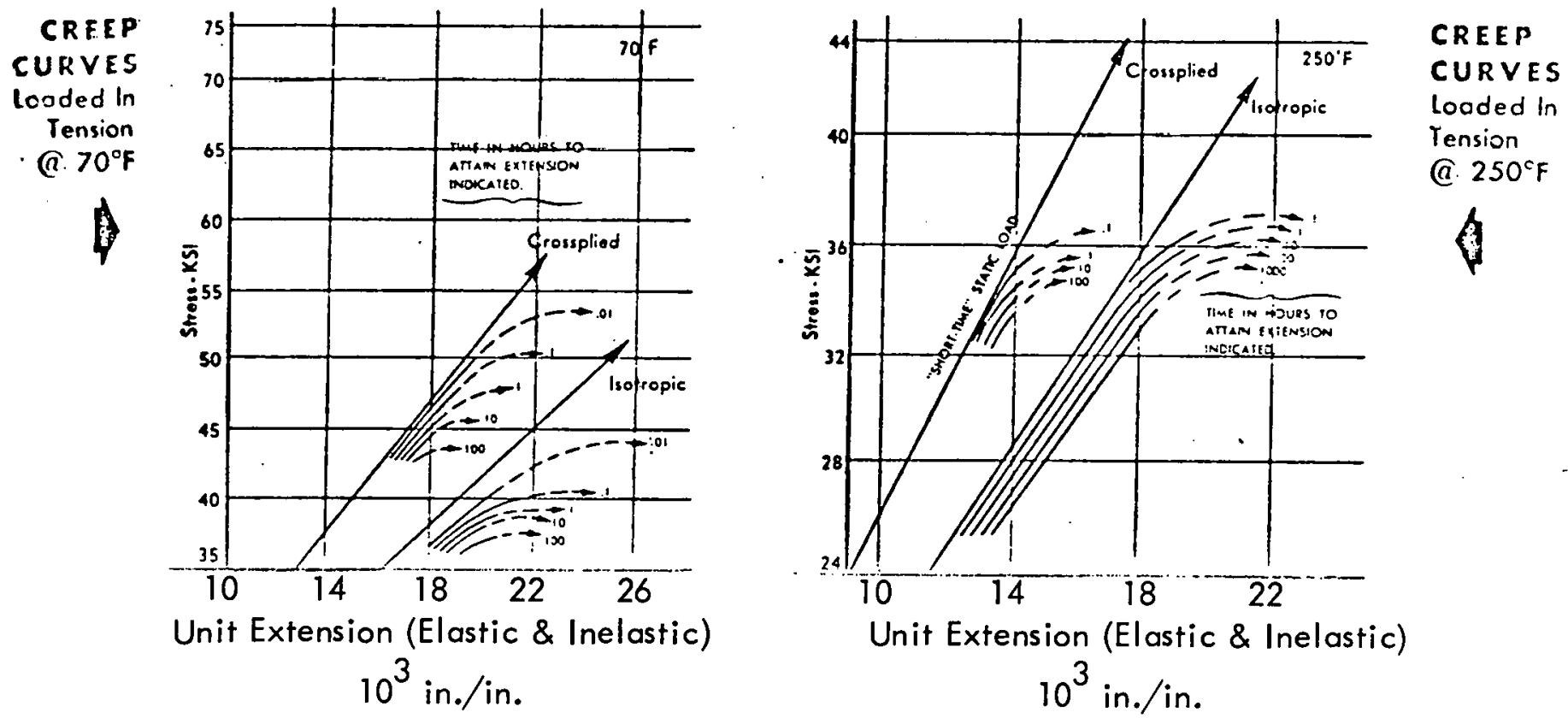

$$
10^{3} \mathrm{in./in.}
$$

\section{STRESS RUPTURE}

Tensile Stress vs. Time to Failure
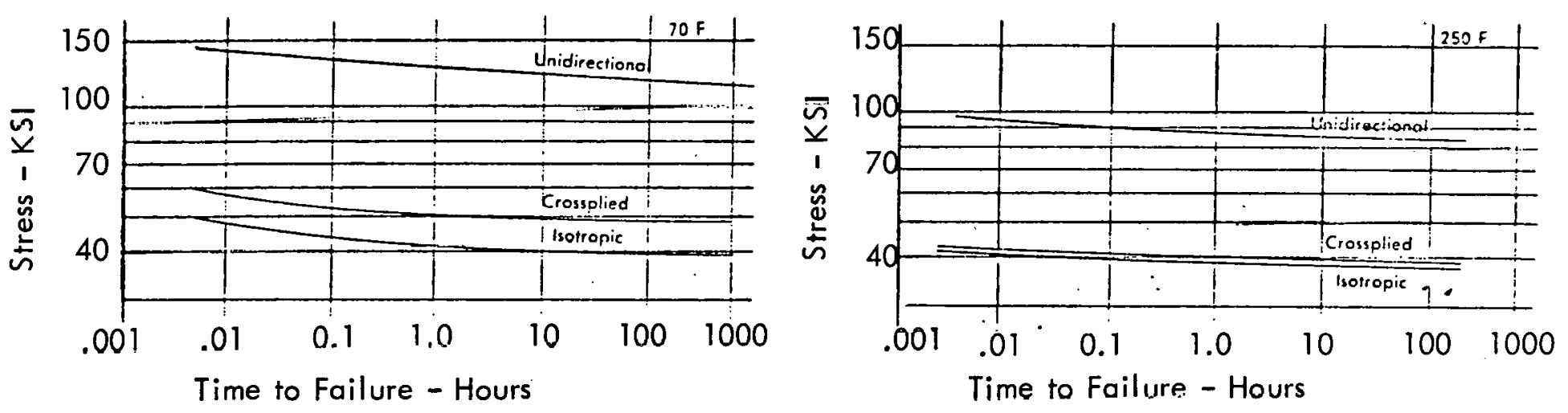

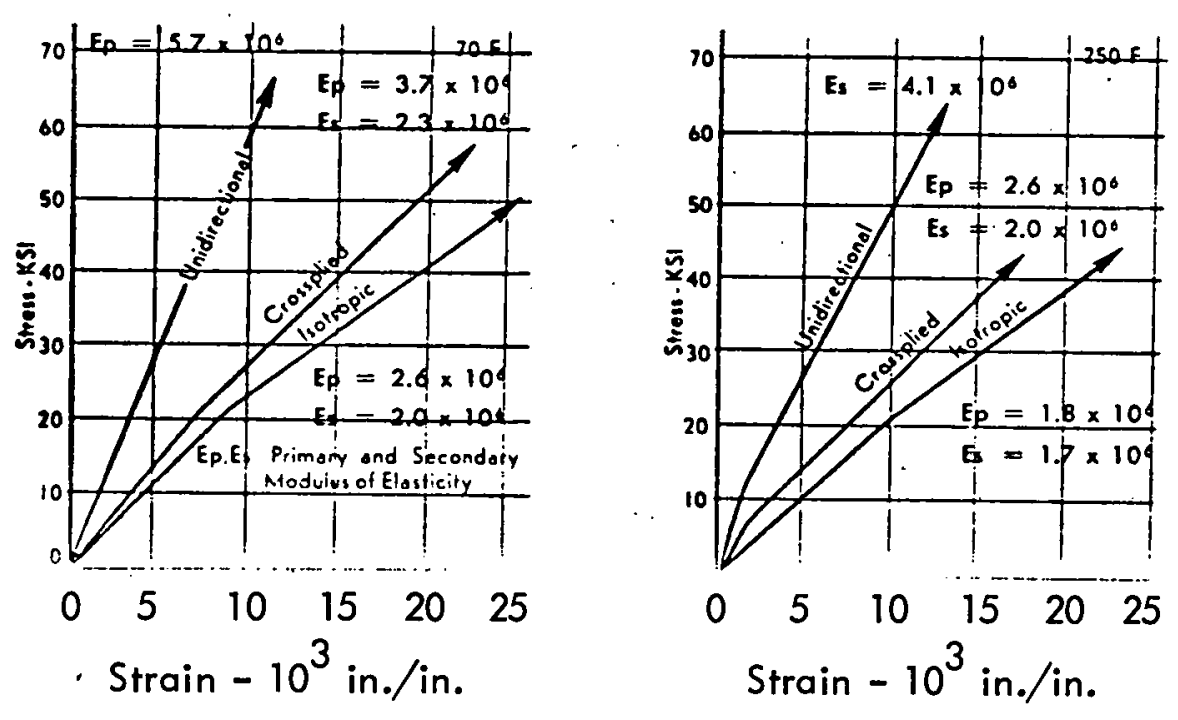

FLEXURAL FATIGUE (SN) CURVES

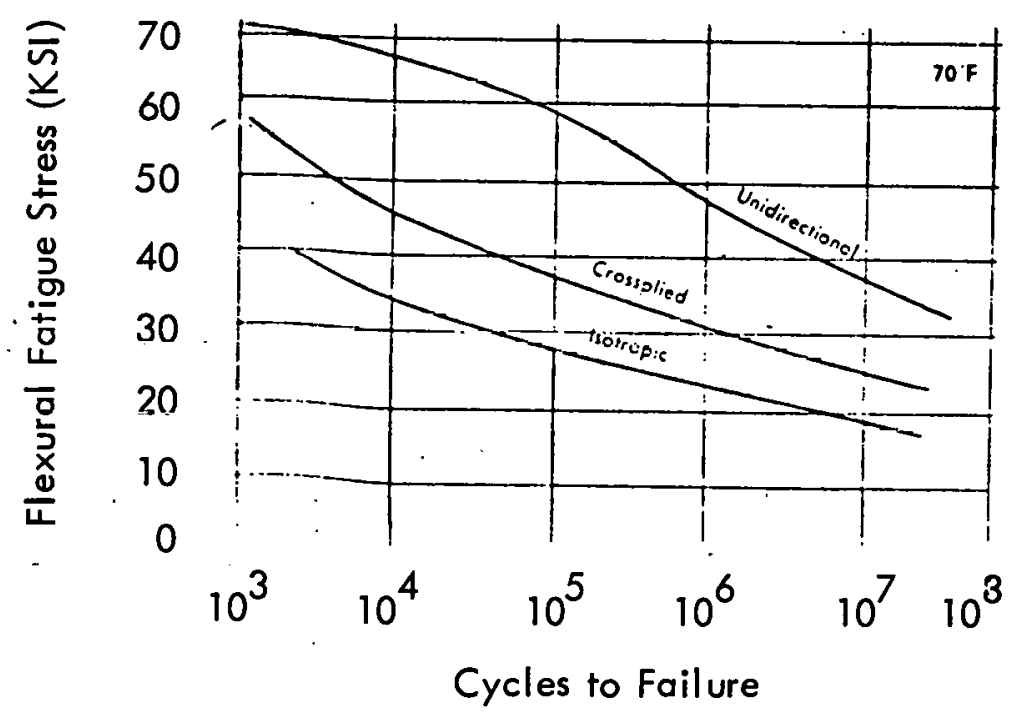


All data from 3M Company Bulletin SP-RSDA (23.5) P. I. dated May 1, 1969.

Since the manufacturer of the product described in this technical data sheet has no means of controlling the final use of the product by the consumer or user, it is the responsibility of the immediate seller or sellers to inform the user of the purposes for which the product may be fit and suitable and of the properties of the product, including the precautionary measures which must be taken in order to insure the safety of the user and of other third persons and property.

All statements, technical information, and recommendations contained herein are based on tests we believe to be reliable but the accuracy or completeness thereof is not guaranteed, and the following is made in lieu of all warranties, express or implied:

Seller's and manufacturer's only obligations shall be to replace such quantity of the product proved to be defective. Neither seller nor manufacturer shall be liable for any injury, loss or damage, direct or consequential, arising out of the use of or the inability to use the product. Before using, user shall determine the suitability of the product for his intended use, and user assumes all risk and liability whatsoever in connection therewith.

No statement or recommendation not contained herein shall have any force or effect unless in agreement signed by officers of seller and manufacturer. 


\subsection{ELECTRICAL TAPE}

SCOTCH NO. 1291

(PULYESTEK HILM WITH ACR YLIC PRESSURE SENJSITIVI ADHESIVE) 
TABLE OF CONTENTS

1:0 GENERAL

2.0 TYPICAL PROPERTIES

3.0 REFERENCE 


\subsection{GENERAL}

"SCOTCH" Brand Electrical Tape 1291 is a two-mil polyester film with an acrylic pressure sensitive adhesive. It is a single-coated tape featuring an adhesive system which offers resistance to most common cleaning solvents, including Freon'. The tape also is resistant to mineral oil, hydraulic fluids, etc., when properly cured. The minimum cure cycle

for oil resistance is 1 hour at $150^{\circ} \mathrm{C}$. The backside of the tape offers excellent printability.

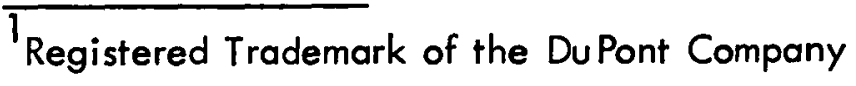




\subsection{TYPICAL PROPERTIES}

\begin{tabular}{|c|c|c|}
\hline Electrical & Units & Value \\
\hline Electrical Strength & Volts & 6500 \\
\hline Insulation Resistance & Megohms & $1 \times 10^{6}$ \\
\hline Electrolytic Corrosion 2 & Ratio & 1.0 \\
\hline Copper Corrosion ${ }^{2}$ & & None \\
\hline \multicolumn{3}{|l|}{ Physical } \\
\hline Caliper & Mils (mm) & $3.2(.088)$ \\
\hline Tensile Strength & lb./in. $(\mathrm{kg} . / \mathrm{cm})$. & $50(8.93)$ \\
\hline Elongation & Percent & 125 \\
\hline Adhesion to Steel & oz./in. (gm./cm.) & $35(391)$ \\
\hline
\end{tabular}

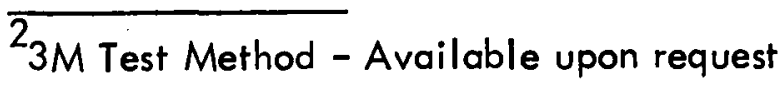




\subsection{REFERENCES}

All data from 3M Company Product Information Bulletin EIP-PI-1291-1 (25.2) R 
2.3 RESIN

(Scotchcast 5(XR-5235) 


\section{TABLE OF CONTENTS}

Title

1.0 GENERAL

2.0 HANDLING PROPERTIES

3.0 TYPICAL PROPERTIES

4.0 PREPARATION

4.1 MIXING

4.2 DEAERATING

4.3 CASTING AND IMPREGNATING

4.4 STORAGE

4.5 CURING

4.6 HANDLING PRECAUTIONS

5.0 REFERENCES 


\subsection{GENERAL}

General purpose impregnating applications designed to operate at $130^{\circ} \mathrm{C}$ (Class "B") temperatures are the intended areas of use for our No. 5 Resin. The distinguishing feature of this rigid system is its excellent resistance to moisture. In addition, this transparent resin offers good physical and electrical properties plus low viscosity for maximum impregnating qualities. 


\subsection{HANDLING PROPERTIES}

MIX RATIO

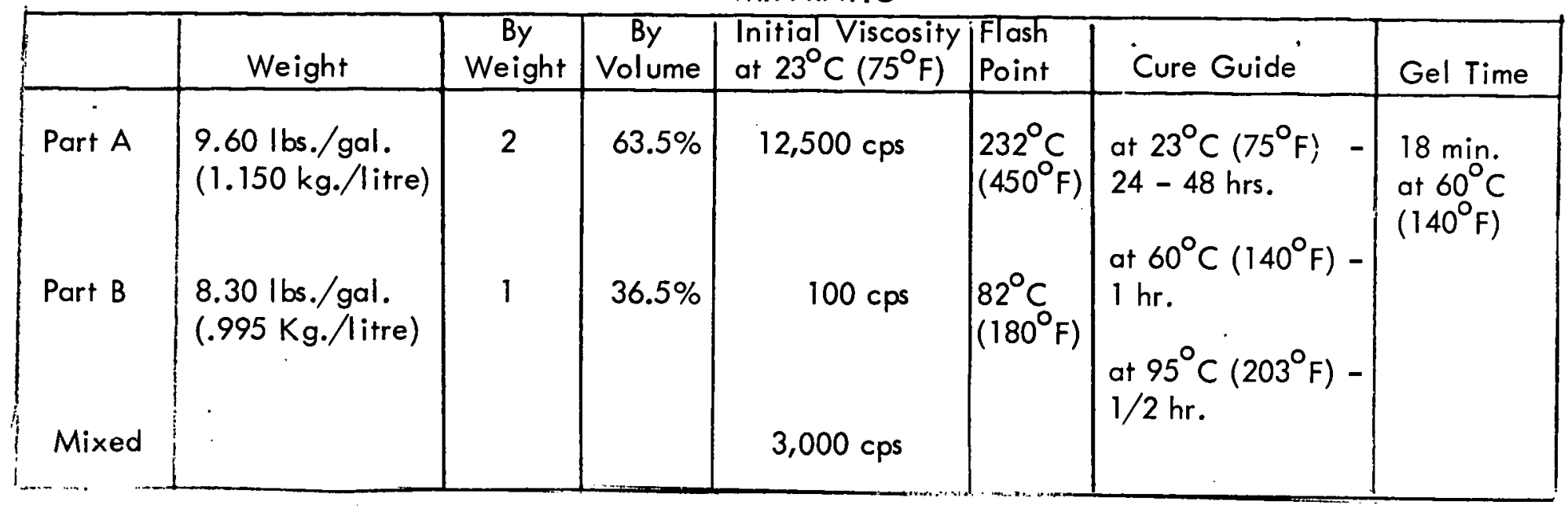




\subsection{TYPICAL PROPERTIES}

PROPERTY

VALUE

\begin{tabular}{|c|c|}
\hline Color & Transparent \\
\hline Specific Gravity & 1.14 \\
\hline Hardness (BARCOL) & 15 \\
\hline Compressive Strength ${ }^{\top}(10 \%$ Compression) & $\begin{array}{l}13,200 \mathrm{PSI} \\
\left(928 \mathrm{Kg} \cdot / \mathrm{cm}^{2}\right)\end{array}$ \\
\hline $\begin{array}{l}\text { Tensile Strength } \\
\text { Ultimate } \\
\end{array}$ & $\begin{array}{l}8000 \mathrm{PSI} \\
\left(562 \mathrm{Kg} . / \mathrm{cm}^{2}\right)\end{array}$ \\
\hline Elongation ${ }^{2}$ (\% at Break) & 7 \\
\hline Flexural Strength $^{3}$ & $\begin{array}{l}12,000 \mathrm{PSI} \\
\left(844 \mathrm{Kg} \cdot / \mathrm{cm}^{2}\right)\end{array}$ \\
\hline Thermal Conductivity ${ }^{4}\left(\mathrm{cal} . / \mathrm{sec} . / \mathrm{cm}^{2},{ }^{\circ} \mathrm{C} / \mathrm{cm}\right)$ & $4.4 \times 10^{-4}$ \\
\hline Linear Thermal Expansion ${ }^{4}$ (length/unit length $/{ }^{\circ} \mathrm{C}$ ) & $177 \times 10^{-6}$ \\
\hline $\begin{array}{l}\text { Moisture Absorption } \\
\% \text { Weight increase, } 95 \% \text { RH, } 240 \mathrm{Hrs} \text {. }\end{array}$ & .5 \\
\hline Boiling Water ( 7 days \% Weight Gain) & 1.8 \\
\hline $\begin{array}{l}\text { Hydrolytic Stability } \\
120 \text { Days } 71^{\circ} \mathrm{C} \\
\left(160^{\circ} \mathrm{F}\right) 95 \% \mathrm{RH} \\
\text { Hardness Loss \% (Shore D) } \\
\end{array}$ & 1.2 \\
\hline Mechanical Shock, Ball Drop Lbs. ${ }^{4}$ & 5 \\
\hline $\begin{array}{l}\text { Thermal Aging } \% \text { Weight Loss } \\
7 \text { days al } 105^{\circ} \mathrm{C} \\
1000 \mathrm{Hrs} \text { at } 130^{\circ} \mathrm{C} \\
1000 \mathrm{Hrs} \text {. at } 155^{\circ} \mathrm{C}\end{array}$ & $\begin{array}{r}.44 \\
3.5 \\
6.6 \\
\end{array}$ \\
\hline Electrical Strength ${ }^{6}$ (Volts/Mil).125" Sample & 325 \\
\hline Dielectric Constant ${ }^{7}\left(1000 \mathrm{~Hz} 23^{\circ} \mathrm{C}\right)$ & 3.6 \\
\hline Dissipation Factor ${ }^{7}\left(1000 \mathrm{~Hz} 23^{\circ} \mathrm{C}\right)$ & .06 \\
\hline Volume Resistivity ${ }^{8}$ ohm- $\mathrm{cm}$ & $10^{14}$ \\
\hline 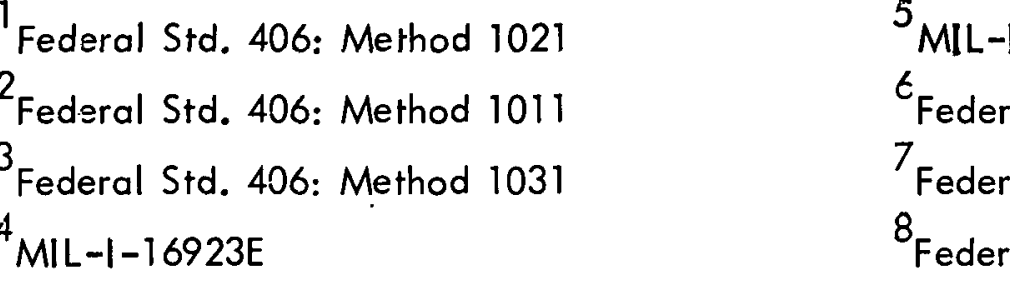 & $\begin{array}{l}{ }^{5} \text { MIL-1-16923G } \\
t_{\text {Federal Std. No. 406: Method } 4031} \\
{ }^{7} \text { Federal Std. No. 406: Method } 4 \\
{ }^{8} \text { Federal Std. No. 406: Method }\end{array}$ \\
\hline
\end{tabular}



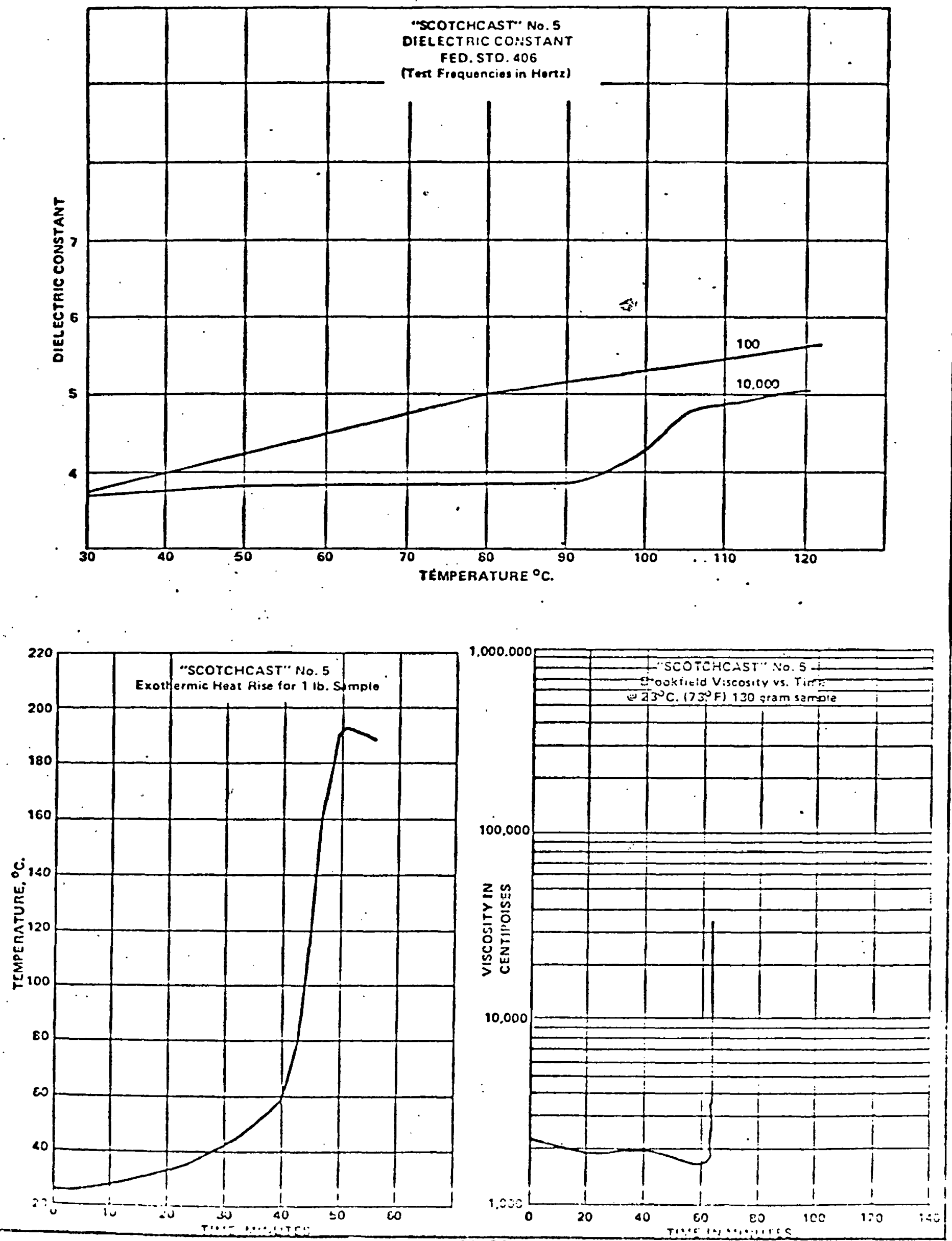

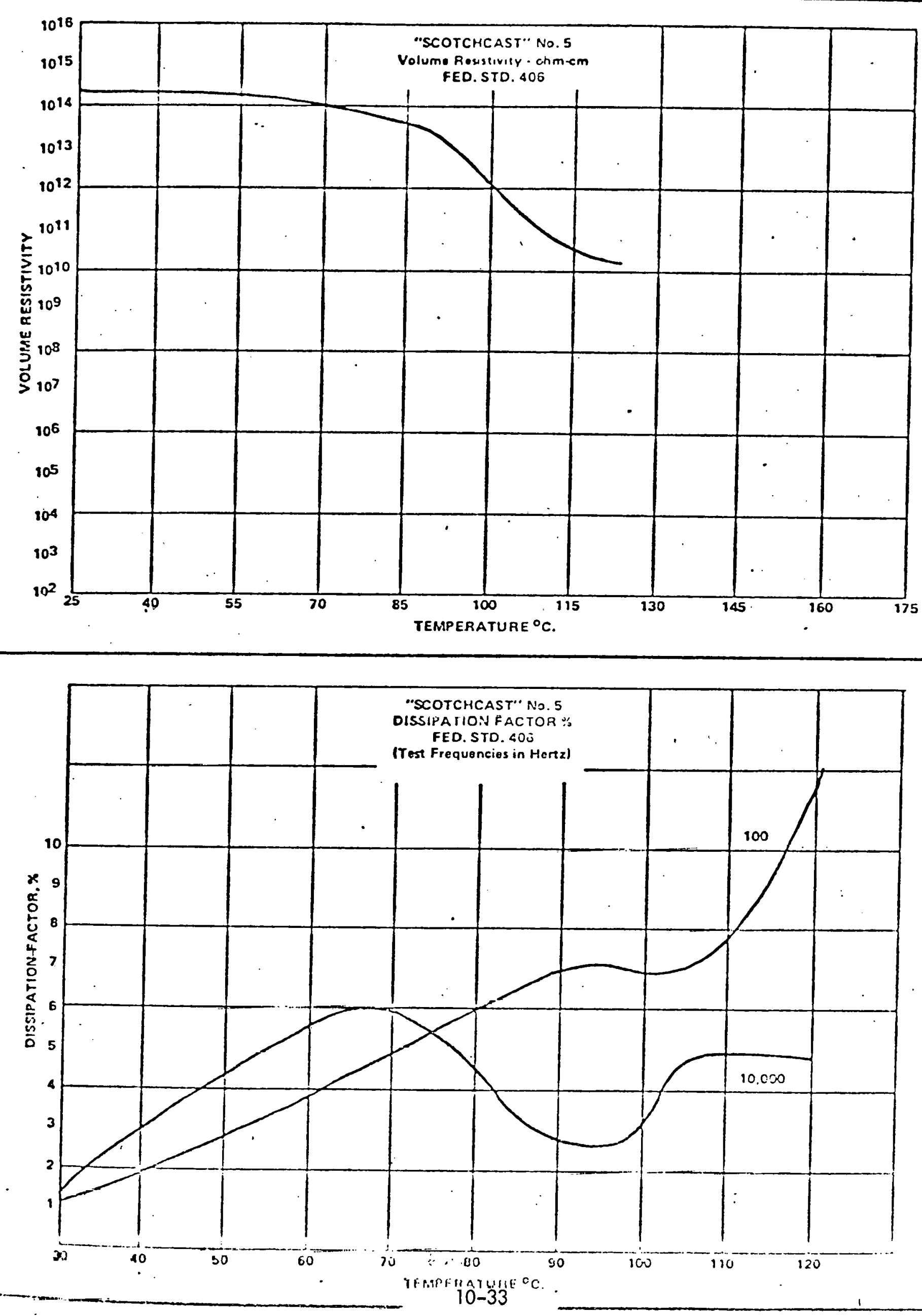


\subsection{PREPARATION}

\subsection{MIXING}

Mix the separate parts before removing them from their containers. They may be warmed to $60^{\circ} \mathrm{C}$. $\left(140^{\circ} \mathrm{F}\right.$ ) to aid mixing. (Gel time is approximately 20 minutes at $60^{\circ} \mathrm{C}$. Thoroughly mix together part $A$ and $B$ in the correct proportions. Mix until the color is uniform or a homogeneous mixture is obtained.

\subsection{DEAERATING}

Air introduced during mixing can be removed by evacuating at 5 to $10 \mathrm{~mm}$ of mercury absolute pressure. The resin can be warmed to aid air removal. Container side wall should be four times hight of liquid resin to contain the foaming that takes place under vacuum.

\subsection{CASTING AND IMPREGNATING}

Pour the warm resin into the preheated $\left(100^{\circ} \mathrm{C}\right.$.) mold. If no mold is used, dip the preheated part into the resin. Heating the part, resin and mold aids impregnation. For maximum impregnation, evacuate at $5 \mathrm{~mm}$ of mercury absolute pressure, or pour under vacuum and hold for several minutes before releasing.

\subsection{STORAGE}

This resin system has a minimum shelf life of one year. Both parts should be stored in a cool, dry place. When not in use, containers should be kept tightly closed.

\subsection{CURING}

The resin will cure at room temperature in 24 to 48 hours. Maximum properties can be achieved more quickly by allowing the resin to gel for 2 to 4 hours at $140^{\circ} \mathrm{F}$. Thin resin sections can be cured in 2 to 4 hours at $140^{\circ} \mathrm{F}$. Castings, which require a large mass of resin, 
should be poured in several layers to minimize the temperature rise due to exotherm. The heat produced by a large mass of this resin might otherwise cause the hardener to volatilize leaving bubbles in the casting. The resin cures to a transparent yellow.

\subsection{HANDLING PRECAUTIONS}

Many of the reactive materials used with epoxy resins have been reported to cause skin irritation to sensitive persons. The user is cautioned to avoid contact with the resin and hardener. The use of protective clothing is recommended. If contact occurs, the skin should be washed immediately with mild soap and water. In case of eye contact, flush immediately with water and secure medical attention. Use only in well-ventilated areas and avoid prolonged or repeated breathing of vapors. 


\subsection{REFERENCES}

All of the information is from 3-M Products Information Bulletin EIP-RPI-5235 (34.2)R.

All statements, technical information and recommendations contained herein are based on tests we believe to be reliable, but the accuracy or completeness thereof is not guaranteed, and the following is made in lieu of all warranties, express or implied:

Seller's and manufacturer's only obligation shall be to replace such quantity of the product proved to be defective. Neither seller nor manufacturer shall be liable for any injury, loss or damage, direct or consequential, arising out of the use of or the inability to use the product. Before using, user shall determine the suitability of the product for his intended use, and user assumes all risk and liability whatsoever in connection therewith.

No statement or recommendation not contained shall have any force or effect unless in an agreement signed by officers of seller and manufacturer. 